MARÍA ROSARIO POLOTTO

THORSTEN KEISER

THOMAS DUVE (EDS.)

\title{
Derecho privado y modernización
}

América Latina y Europa en la primera mitad del siglo XX

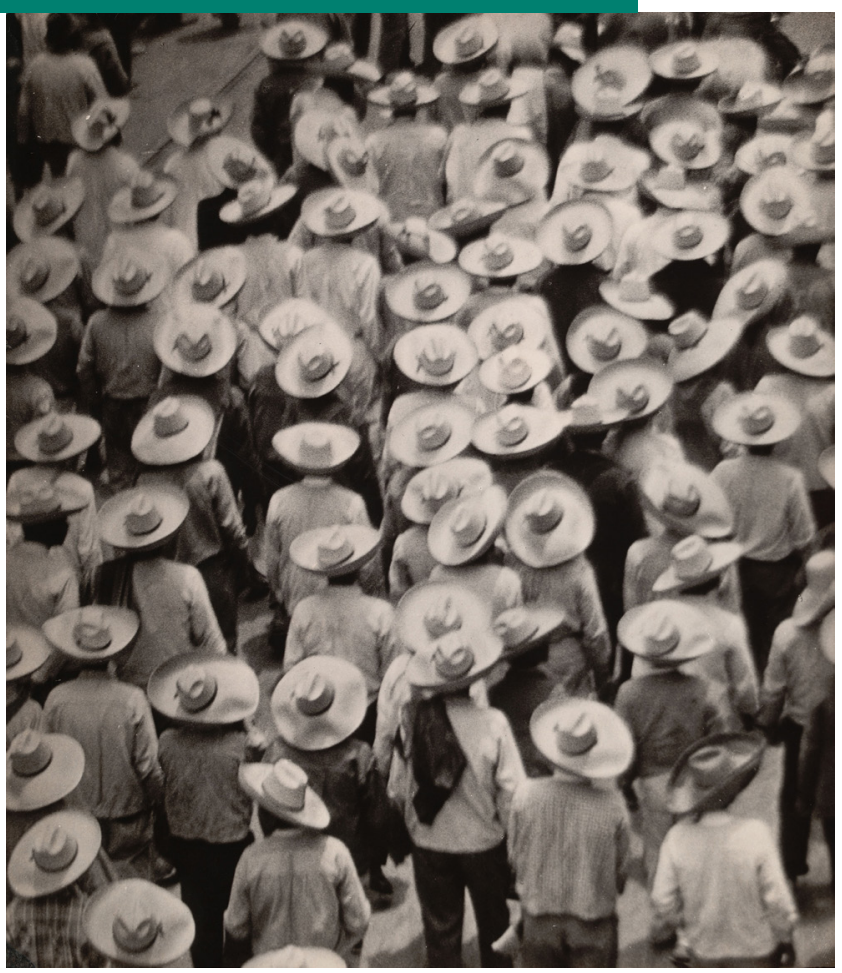



GLOBAL PERSPECTIVES

ON LEGAL HISTORY 


\title{
Global Perspectives on Legal History
}

\author{
A Max Planck Institute for European Legal History \\ Open Access Publication
}

http://global.rg.mpg.de

Series Editor: Thomas Duve

Volume 2

Global Perspectives on Legal History is a book series edited and published by the Max Planck Institute for European Legal History, Frankfurt am Main, Germany.

As its title suggests, the series is designed to advance the scholarly research of legal historians worldwide who seek to transcend the established boundaries of national legal scholarship that typically sets the focus on a single, dominant modus of normativity and law. The series aims to privilege studies dedicated to reconstructing the historical evolution of normativity from a global perspective.

It includes monographs, editions of sources, and collaborative works. All titles in the series are available both as premium print-on-demand and in the open-access format. 
MARÍA ROSARIO POLOTTO

THORSTEN KEISER

THOMAS DUVE (EDS.)

\section{Derecho privado y modernización}

América Latina y Europa en la primera mitad del siglo XX

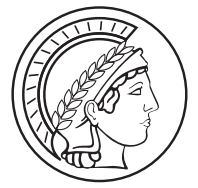

MAX PLANCK INSTITUTE

FOR EUROPEAN LEGAL HISTORY

2015 
ISBN 978-3-944773-01-8

eISBN 978-3-944773-11-7

ISSN 2196-9752

First published in 2015

Published by Max Planck Institute for European Legal History, Frankfurt am Main

Printed in Germany by epubli, Prinzessinnenstraße 20, 10969 Berlin

http://www.epubli.de

Max Planck Institute for European Legal History Open Access Publication http://global.rg.mpg.de

Published under Creative Commons CC BY-NC-ND 3.0 DE

http://creativecommons.org/licenses/by-nc-nd/3.0/de

The Deutsche Nationalbibliothek lists this publication in the Deutsche Nationalbibliographie; detailed bibliographic data are available on the Internet at http://dnb.d-nb.de

Copyright (c)

Cover photo by Modotti, Tina: Workers Parade, 1926 (C) 2015

The Museum of Modern Art, New York/Scala, Florence

Cover design by Elmar Lixenfeld, Frankfurt am Main

Recommended citation:

Polotto, María Rosario, Keiser, Thorsten, Duve, Thomas (eds.) (2015), Derecho privado y modernización. América Latina y Europa en la primera mitad del siglo XX,

Global Perspectives on Legal History, Max Planck Institute for European Legal History

Open Access Publication, Frankfurt am Main, http://dx.doi.org/10.12946/gplh2 


\section{Contents}

1 | María Rosario Polotto, Thorsten Keiser, Thomas Duve Introducción

11 | Alessandro Somma

Le parole della modernizzazione latinoamericana. Centro, periferia, individuo e ordine

47 | Marcelo Neves

Ideas in Another Place? Liberal Constitution and the Codification of Private Law at the Turn of the $19^{\text {th }}$ Century in Brazil

83 | Maria Rosa Pugliese

La denominada «crisis del derecho» desde la perspectiva argentina durante el periodo de entre guerras mundiales (1920-1940)

119 | Alfons Aragoneses

Crisis del derecho privado y legislación especial en Francia y en Argentina

153 | Daniela Marino Institutionalización de la Reforma Agraria (1915-1937).

Revolución y modernización jurídica en México

179 | Alfredo de J. Flores

El proyecto de modernización del ideario liberal republicano en Brasil en cuestión: las ediciones de la "Consolidação das leis civis» durante la «República Velha» (1889-1930)

199 | Gustavo Silveira Siqueira

Republic and Strike Action in the Beginning of the $20^{\text {th }}$ Century:

A Debate between the 1906 Strike and Legal History 
211 | Yolanda de Paz Trueba

Beneficencia católica, Estado municipal e infancia.

Una forma de intervención pública en el ámbito privado de la familia a fines del siglo XIX

227 | Agostina Gentili

Un fuero híbrido: Juzgados de menores, precedentes y prácticas en Córdoba, Argentina, primera mitad del siglo XX

245 | María Rosario Polotto

Un codigo para el desierto argentino. La discusión en torno a la propiedad del ganado en el discurso académico de la primera mitad del siglo XX

275 | Mario Losano

Tra Uruguay e Italia: Couture e Calamadrei, due giuristi democratici nell'epoca delle dittature europee

313 | Contributors 


\section{María Rosario Polotto \\ Thorsten Keiser \\ Thomas Duve}

\section{Introducción}

Los trabajos que reúne este volumen son el resultado de la reflexión y discusión que un grupo de investigadores, adscriptos a distintas corrientes disciplinares, llevaron adelante, bajo la iniciativa del Max-Planck-Institut für europäische Rechtsgeschichte y el Instituto de Investigaciones de Historia del Derecho, en el Seminario «Derecho Privado y Modernización en América Latina y Europa en la primera mitad del siglo XX» que se celebró en Buenos Aires del 4 al 6 de julio de 2012.

Las implicancias y consecuencias de los procesos modernizadores que sumieron, en los siglos XIX y XX, a los estados de los cinco continentes, ha sido objeto de estudio desde los más diversos enfoques disciplinares. De ahí que el objetivo principal de esta reunión académica fuese encontrar nuevas vías de investigación que permitieran indagar, describir y profundizar el impacto que dichos procesos ejercieron sobre las representaciones y conceptos del derecho privado moderno tanto europeos como latinoamericanos. Para ello se primó en el análisis una perspectiva comparada que permitiera superar, especialmente en los trabajos iushistoriográficos, una visión eurocéntrica de estos fenómenos.

1. Los estudios sociales hacen referencia a la «modernidad» y a la «modernización» como categorías funcionales, que sin significar necesariamente lo mismo, permiten la comparación de diversas realidades históricas.

Acuñadas sus primeras significaciones en la crítica estética y desarrolladas luego en el campo de la filosofía y de la sociología, ${ }^{1}$ el concepto de la modernidad ha estado frecuentemente condicionado desde una percepción formada en la experiencia europea. ${ }^{2}$ Se la identificó fundamentalmente

1 Distintos autores atribuyen a Baudelaire, a mediados del siglo XIX, un nuevo significado semántico del término «modernidad»: Habermas (2008), 19; Delanty (2011), 408-409.

2 Bauman (2005), 160. 
como «la objetivación histórica de estructuras racionales» ${ }^{3}$ que llevaron a destacar la «superioridad» de esta etapa histórica en la medida que implicaba la «subordinación, marginación, erradicación o aniquilación de sus alternativas premodernas». ${ }^{4}$

Por otro lado, el paradigma de la modernización tuvo un notable desarrollo conceptual durante las décadas de los cuarenta y cincuenta del siglo pasado, impulsado por el optimismo de cierto sector de la intelectualidad anglosajona de la segunda postguerra, que destacaba a Estados Unidos como la sociedad más democrática y estable del mundo. ${ }^{5}$ En este contexto se la concibió como un proceso inmanente, de «maduración obligada» del sistema social, por el cual las sociedades pasan de una fase «tradicional» caracterizada por el particularismo, a una fase «moderna» caracterizada por valores universalistas, la búsqueda de la eficacia y del logro en la acción, y la especificidad funcional, liderada ésta por los Estados Unidos. ${ }^{6}$ Refería a un «manojo de procedimientos acumulativos» que se reforzaban mutuamente: «a la formación del capital y a la movilización de recursos, al desarrollo de las fuerzas productivas y al incremento de la productividad del trabajo, a la implantación de poderes políticos centralizados y al desarrollo de entidades nacionales, a la difusión de los derechos de participación política, de las formas de vida urbana y de la educación formal, a la secularización de valores y normas, etc». ${ }^{7}$

Como lo ha señalado Habermas la teoría de la modernización implicó una abstracción del concepto de modernidad, desgajando a ésta de «sus orígenes moderno europeos para estilizarla y convertirla en un patrón de procesos de evolución social neutralizados en cuanto al tiempo y al espacio». ${ }^{\mathbf{8}}$

Nuevos paradigmas hoy intentan explicar la modernidad y los procesos de modernización superando los esquemas rígidos que caracterizaron las anteriores descripciones. Así los conceptos de «modernidades múltiples» ${ }^{9}$ «modernidad reflexiva» abren las puertas para nuevas aportaciones. ${ }^{\mathbf{1 0}}$ Otros

3 Habermas (2008), 12.

4 Bauman (2005), 166.

5 Gimenez (1995), 38.

6 Gimenez (1995), 37-38; Parsons (1985), 330-331.

7 Habermas (2008), 12.

8 Habermas (2008), 12.

9 Eisenstadt (1987).

10 Gimenez (1995), 40; Giddens et al. (1996); BecK/Giddens / Lash (2001). 
implican una critica fundamental de los relatos de una modernidad de orientación occidental desarrollando nuevas representaciones para los fenómenos en estudio. Al mismo tiempo una critica se dirige al enfoque cronológico lineal de la historia, que estaba conectado inseparablemente con las narrativas de modernización. ${ }^{\mathbf{1 1}}$

2. Para el caso latinoamericano no han faltado ni faltan tentativas para describir la vía de la modernidad. ${ }^{\mathbf{1 2}}$ En este tópico el debate no ha sido sólo profuso sino también enriquecedor. Desde la reflexión sociológica hasta el análisis literario, pasando por los nuevos enfoques de quienes estudian los procesos políticos en la génesis de los estados americanos, se ha tratado de delinear el significado de un discurso modernizador por parte de las elites latinoamericanas y su impacto en la realidad política, social, económica y cultural.

Por un lado se ha descripto al espacio latinoamericano como una articulación "compleja de tradiciones y modernidades», donde coexisten en los distintos países que lo forman «múltiples lógicas de desarrollo» que dan lugar a esa «heterogeneidad multitemporal», a esas "culturas híbridas» que caracterizan a la cultura moderna en este continente. ${ }^{13}$ De lo que se sigue la necesidad de superar ciertas visiones que hacen de los procesos modernizadores latinoamericanos una versión deficiente de sus modelos europeos, así como aquellas que exaltan la originalidad de estas culturas. ${ }^{\mathbf{1 4}}$

Los aportes de la historia de los «lenguajes políticos» han ayudado no solo al replanteamiento de todo el esquema de «modelos» y «desviaciones» sino también a franquear una concepción «genealógica» $\mathrm{o}$ «teleológica» del problema de la circulación de ideas entre Europa y América. Al destacar el carácter contingente de los discursos rescató la necesidad no sólo de descubrir los «umbrales de historicidad» inherentes a ellos, sino también la exigencia de reconstruir los contextos en los que ellos son formulados. Así, la modernidad no se identificaría con un único modelo social o tipo ideal, para

11 Hoffmann (2005).

12 Como un resumen actual p. e.: Miller/Hart (2007).

13 García Canclini (2010), 37, 43 y 86.

14 Para este tema resulta sumamente interesante recordar el debate suscitado, en los años 70 , por Roberto Schwarz con sus «ideas fuera de lugar», Schwarz (1977). Para un análisis de la cuestión ver también García Canclini, (2010), 87-94 y Palti (2007b). 
muchos autores referenciado al europeo, sino que «comprendería diversas alternativas posibles». ${ }^{\mathbf{1 5}}$

La invitación consistiría en recuperar la «dimensión propiamente política» de las ideas, en la medida que los actores políticos locales intentaron «articular concretamente respuestas prácticas a problemas para los cuales no existían en realidad soluciones a priori válidas o incontestables»; ${ }^{\mathbf{1 6}}$ en revalorizar el papel de las élites latinoamericanas en la «especificidad de sus posiciones ante la tarea de construir un país moderno», cuyo resultado es un «texto formado por capas ideológicas y yuxtapuestas, esto es, por componentes teóricos heterogéneos y a menudo sin mejor armonización que la que puede conferirles el proyecto político en el que convergen». ${ }^{\mathbf{1 7}}$

3. Resta por último profundizar la cuestión de la modernidad y modernización desde una perspectiva jurídica, cuestión que fuera el objetivo principal del Seminario indicado al comienzo de esta introducción y de los trabajos que reúne este volumen.

En primer lugar cabe señalar una primera dificultad que se ha verificado en el discurso jurídico de la época estudiada constituye la ausencia de una referencia explícita a dichos conceptos. Una excepción podría ser el uso del término «modernismo jurídico» - concepto en sí ambiguo - en el lenguaje jurídico italiano de los años treinta. ${ }^{\mathbf{1 8}}$ Sin embargo, es posible entrever un «derecho moderno» en expresiones que en el período estudiado aludían a un derecho nuevo, como por ejemplo, «social», «colectivista» o «solidarista». En todo caso éstas se encuentran íntimamente relacionadas con las percepciones de una crisis del derecho liberal decimonónico, ${ }^{19}$ y con la insuficiencia de éste para afrontar los nuevos desafíos que imponían las transformaciones

15 Palti (2007b), 51, 54-56.

16 Palti (2008), 34.

17 «Las circunstancias específicas e irrepetibles de emisión y circulación discursivas hacen que nuestros intelectuales no puedan no haber sido originales, a su manera. Han generado una resemantización sudamericana de conceptos ya presentes en el modelo de pertenencia, han creado otros y, en todo caso, han dado respuestas autóctonas a requisitos planteados por situaciones históricas peculiares, enriqueciendo de ese modo el modelo mismo». DotTi (1990), 12-14.

18 El primer jurista que utilizó el término fue Cesarini Sforza, Grossi (2000), 163. AragoNESES (2009), 49.

19 Donde es de notar que las referencias a la modernización suelen ir acompañados de observaciones de una crisis. Ver Meyer (ed.) (2013). 
sociales, económicas y políticas de la época. ${ }^{20}$ Uno de los objetivos de este proyecto era advertir los discursos en torno a los cambios legislativos que se suscitaron en el período estudiado y revelar la perspectiva de los actores contemporáneos a los mismos más allá de las construcciones efectuadas a posteriori por la ciencia histórica.

En segundo lugar, a diferencia de lo que ocurre en otras disciplinas, como ya se ha visto, los estudios iushistoriográficos adolecen, en su mayoría, de una mirada crítica de los procesos modernizadores. Muchas de estas descripciones en términos de «circulación de ideas» $\mathbf{o}$ «recepción del derecho extranjero» se enmarcan en el esquema de «modelos» $\mathrm{y}$ "desviaciones» que se ha señalado, al abstraer estos fenómenos de sus contextos políticos, sociales y económicos e incluso ideológicos. Un intento de superar esta visión han sido los estudios sobre la «pervivencia» del derecho indiano en las experiencias codificadoras iberoamericanas, que si por un lado han enriquecido el análisis al evidenciar la operatividad de lógicas tradicionales en amalgama con acciones modernizadoras, por el otro lado no abandonan un enfoque idealista $o$ esencialista del problema. La propuesta de los trabajos que aquí se recogen es justamente no eludir la complejidad de estas cuestiones, apelar a esos «umbrales de historicidad», que nos permitan vislumbrar estos procesos en su realidad histórica concreta.

Un caso especial lo constituyen los operadores jurídicos, aquellos que manipulan el material normativo, y en particular los juristas. La contemplación del flujo de ideas y experiencias desde este peculiar observatorio nos brinda una dimensión insospechada de estos fenómenos. Lo que parece procesos mecánicos, guiados por una lógica determinista de modernización, se convierten en encuentros casuales, relaciones epistolares, migraciones forzadas, lecturas fortuitas, viajes académicos, y por qué no, amistades que duran toda la vida. Llegamos a una reconstrucción capilar de estas problemáticas, donde la magnitud de lo subjetivo y lo azaroso resulta relevante. Estas redes académicas no aparecen con una dirección única de circulación (del centro a la periferia), sino que nos enfrentamos a fenómenos que muchas veces se presentan como de «vinculaciones horizontales», en un escenario de simultáneas emisiones y recepciones. ${ }^{21}$

20 Como ejemplo de otra historia de la modernización a traves un derecho liberal en el siglo 19 ver SCHERNER/Bergfeld (1993).

21 Aвásolo (2014). 
4. Hechas estas consideraciones pasamos a una presentación de los trabajos aquí publicados.

A través de la comparación de la experiencia francesa y argentina, Alfons Aragoneses reconstruye en detalle el peculiar ambiente en el que se debatió las leyes de accidentes de trabajo en sendos países. Analizando los diversos actores que participaron en las discusiones (juristas, legisladores, asociaciones empresariales, académicos, sindicatos, etc.) así como las diversas propuestas legislativas y doctrinales, el autor describe un complejo flujo de ideas y conceptos que resulta imposible caracterizarlo como unidireccional. Concluye que entre ambas experiencias nacionales existiría una identidad terminológica de leyes, expresiones o instituciones europeas y argentinas, pero no necesariamente una identidad semántica, lo que no permitiría encuadrar la cuestión como una simple recepción o importación y adaptación de textos legislativos sino una verdadera creación por parte de las elites argentinas de nuevas ideas, de nuevas instituciones e incluso del Estado y la sociedad.

Alfredo Flores nos sumerge en el problema de la adaptación de las ideas jurídicas liberales en el período de la República que comprende los años 1889-1930. Para ello llama la atención sobre las diversas ediciones que tuvo la Consolidação das leis civis de Augusto Teixeira de Freitas, obra que de características tradicionales y que remitía al viejo derecho colonial, abierto ya el proceso de codificación civil. Para el autor estas reimpresiones, que datan de los años 1896, 1910 y 1915, estarían impuestas por la receptividad que el texto tenía en la práctica forense y cumpliría con las necesidades del foro, originándose una disparidad entre el discurso y la propuesta liberal de la República y la pervivencia en los procedimientos judiciales de elementos tradicionales.

A través del abordaje concienzudo tanto de su organización como de su praxis, Agostina Gentili nos introduce en los avatares de la implementación de los Juzgados de Menores en Córdoba durante la primera mitad del siglo XX. El estudio revela no solo el manejo que los expertos tenían de las experiencias europeas, sino también esa circulación horizontal de modelos e ideas que dábamos cuenta anteriormente. La modernización institucional argentina se enfrentaba a importantes desafíos, siendo uno de ellos la estructura federal de nuestro país, que condicionaba la administración de justicia. De ahí que la creación de esta magistratura en Córdoba estuviese supeditada no solo por los lineamientos impuestos por la Ley Nacional de Patronato de Menores, sancionada en 1919, sino también por las prácticas diseñadas en la 
Provincia de Buenos Aires, principal modelo en la materia. Si a ello sumamos los contratiempos políticos y económicos que influyeron en su construcción, resulta justificada la calificación de «híbrida» con que la autora caracteriza esta institución.

El trabajo de Mario Losano revela la constitución capilar de las relaciones intelectuales entre Uruguay e Italia, demostrándonos que éstas difícilmente pueden ser conceptualizadas bajo argumentos de dependencia cultural o recepción pasiva de ideas. Las mismas, cuyo entramado pudo ser recuperado gracias a la compulsa del Archivo Couture en Montevideo, tienen su epicentro en los grandes juristas ubicados a cada lado del Atlántico: Eduardo J. Couture y Piero Calamandrei. A través de su «mediación humanitaria y cultural», basada en la mutua admiración y el «amor por los estudios comunes», ambos permitieron la formación de una amplia red de académicos que, en el contexto dramático y doloroso de la guerra, pudieron emigrar hacia Sudamérica. Esta «amicizia operosa» fue alimentada, por décadas, por un intercambio epistolar que incluyó no solo estos dos procesalistas, sino también a Enrico Tullio Liebman, Tullio Ascarelli, Renato Treves, Leone Lattes y Giorgio Del Vecchio entre otros, y que da testimonio el archivo mencionado.

Profundizando su trasfondo social y político, así como sus fuentes normativas y la particular visión de los juristas, Daniela Marino problematiza aquellas perspectivas que ven en la Constitución mexicana de 1917 y, en particular, en la institucionalización de la reforma agraria, hitos precursores de desarrollos políticos y jurídicos posteriores en otras partes del mundo. En este contexto aborda el régimen de la propiedad con el objetivo de indagar no sólo sus cambios y permanencias, sino también la influencia en su desarrollo de circunstancias locales y políticas y de procesos extra-mexicanos, como serían aquellos aportes provenientes de la «socialización del derecho» y el intervencionismo estatal. Así la autora concluye que si bien la Revolución introdujo cambios al forzar medidas de retribución social a grandes sectores de la población, éstos no fueron suficientemente innovadores. Solamente el recambio de la vieja estructura burocrática y académica liberal positivista harían realidad los principios sociales sostenidos por la Revolución y consagrados en la Constitución de 1917.

Siguiendo los postulados de la Teoría de la Comunicación de Luhmann, Marcelo Neves examina las semánticas liberales contenidas en el constitucionalismo y codificación civil brasilera de los siglos XIX y principios del XX. 
Para este autor las ideas liberales no se encuentran «fuera de lugar», como sostienen importantes pensadores de ese país, sino que por el contrario, las mismas pertenecen al mundo semántico de la sociedad en la que circulan, al manifestar una importante dimensión normativa más allá de su concretización en las experiencias históricas estudiadas. Esto se resume en una función simbólica de éstas que permitía, en forma ambigua, negar y reforzar o expandir los derechos.

En el marco de la organización del estado nacional y provincial en la Argentina, Yolanda de Paz Trueba llama la atención sobre la persistencia de estructuras tradicionales y corporativas para dar respuesta a distintas cuestiones sociales, como es el caso de la niñez desvalida. Mediante la compulsa de los archivos y reglamentos de los Asilos dirigidos por las Sociedades de Damas de Caridad del Sagrado Corazón de Jesús en los pueblos de Tandil y Azul, en la Provincia de Buenos Aires, la autora revisa la actuación de esta institución en la acogida y egreso de estas menores, develando prácticas que se mantienen incluso en contradicción con el marco normativo impuesto por el mismo Código Civil.

Las condiciones políticas y económicas imperantes en un determinado país se erigen muchas veces como vallas para la implementación de los criterios de uniformidad, racionalidad y sistematización que acompañan a los códigos decimonónicos impuestos por el Estado. En su estudio, María Rosario Polotto, identifica la estructura federal argentina y el peculiar régimen de propiedad del ganado heredado de la colonia, como elementos que entran en contradicción con la solución codificada inspirada en el derecho francés. El análisis reconstruye el esfuerzo argumentativo de los expertos del derecho por conciliar ambos extremos, elaborando doctrinas que sin negar la centralidad del Código Civil, permitiera la pervivencia de ciertas reglas normativas que se adecuaban mejor a la praxis económica de la República.

Centrándose en el tópico de "crisis del derecho», María Rosa Pugliese pasa revista de las principales problemáticas que ocuparon a los juristas argentinos del período de entre guerras. Desde este especial punto de observación, la autora da cuenta de la crítica al derecho decimonónico y de las nuevas concepciones en torno al derecho social, el rol de la jurisprudencia, la intervención del Estado, la reforma de la legislación y del Código Civil. Remarca también que en la construcción de las argumentaciones no sólo tuvieron peso importante la realidad argentina, sino además la profusa circulación de ideas, en la cual tuvo un rol destacado las revistas jurídicas, las que sirvieron 
de canal privilegiado para el conocimiento de los discursos y experiencias tanto americanas como europeas.

El trabajo de Gustavo Silveira Siqueira nos invita a recordar que el estudio histórico de las normativas y leyes no pueden ser abarcado en términos de evolución, sino mediante la recreación del contexto y la complejidad que las caracterizaron. A través del análisis de los pormenores que llevaron a la huelga ferroviaria en el estado brasileño de Saõ Paulo, indaga, recurriendo a distintas fuentes, la configuración del derecho de huelga en esa época y su valoración en la cultura jurídica local.

Alessandro Somma nos aporta un examen crítico de los distintos esquemas analíticos iuscomparatistas que afianzaron una imagen periférica del derecho latinoamericano. Apelando al contexto histórico en que surgieron y a sus elementos ideológicos, pone el autor en evidencia, que los modelos interpretativos centrados en el cambio jurídico, como el de «familias jurídicas», «new comparative economics» o el de «tradición jurídica occidental» afianzaron una representación del derecho latinoamericano como dependiente de la experiencia europea o norteamericana y receptor pasivo del derecho proveniente del «centro» de la cultura, que se imponía por su originalidad y admiración que producían. De esta manera se afirmaba la superioridad del derecho occidental como motor de la modernización, entendida ésta como la construcción de una sociedad capitalista y democrática. Otras explicaciones posibles, intento de superar esta visión, serían el de la originalidad y el de las modernidades múltiples. En definitiva el autor busca destacar la fragilidad del nexo entre democracia y capitalismo como elementos distintivos de la modernización y del derecho occidental como vehículo de ese nexo.

\section{Bibliografía}

Aвásolo, Ezequiel (2014), Aportes del comparatismo jurídico al estudio de la circulación de ideas y experiencias normativas en Europa y América durante la primera mitad del siglo XX, en: ABásolo, Ezequiel (Director), La cultura jurídica latinoamericana y la circulación de ideas durante la primera mitad del siglo XX. Aproximaciones teóricas y análisis de experiencias, Buenos Aires: Instituto de Investigaciones de Historia del Derecho, 11-21

Aragoneses, Alfons (2009), Un jurista del Modernismo. Raymond Saleilles y los orígenes del derecho comparado, Madrid: Dykinson 
Bauman, Zygmunt (2005), Legisladores e intérpretes. Sobre la modernidad, la posmodernidad y los intelectuales, traducción Horacio Pons, 1a reempresión, Buenos Aires: Universidad Nacional de Quilmes

Beck, Ulrich, Anthony Giddens, Scott Lash (2001), Modernización reflexiva. Política, tradición y estética en el orden social moderno, Madrid: Alianza

Delanty, Gerard (2011), Modernity. en: Ritzer, George, J. Michael Ryan (ed.), The Concise Encyclopedia of Sociology, Chicester: Blackwell, 408-409

Dotti, Jorge (1990), Las vetas del texto. Una lectura filosófica de Alberdi, los positivistas, Juan B. Justo, Buenos Aires: Punto Sur Editores

Eisenstadt, Shmuel, Patterns of modernity, New York: New York University Press 1987

García Canclini, Néstor (2010), Culturas híbridas. Estrategias para entrar y salir de la modernidad, Nueva edición, 3era. reimpresión, Buenos Aires: Paidós

Giddens, Anthony et al. (1996), Las consecuencias perversas de la modernidad. Modernidad, contingencia y riesgo, Josetxo Beriain (Comp.), traducción de Celso Sánchez Capdequí, Barcelona: Editorial Anthropos

Gimenez, Gilberto (1995), Modernización, Cultura e Identidad Social, en: Espiral, Estudios sobre Estado y Sociedad I, 2 (Enero Abril), 35-56

Grossi, Paolo (2000), Scienza giuridica italiana. Un profilo storico, 1860-1950, Milano: Giuffrè

Habermas, Jürgen (2008), El discurso filosófico de la modernidad, traducción Manuel Jiménez Redondo, Buenos Aires: Katz Editores

Hoffmann, Arnd (2005), Zufall und Kontingenz in der Geschichtstheorie, Frankfurt/M.: Klostermann

Meyer, Carla (ed.) (2013), Krisengeschichte(n). «Krise» als Leitbegriff und Erzählmuster in kulturwissenschaftlicher Perspektive, Stuttgart: Steiner

Miller, Nicola, Stephen Hart (ed.) (2007), When was Latin America modern? New York: Palgrave

Palti, Elías (2007a), El tiempo de la política. El siglo XIX reconsiderado, buenos Aires: Siglo Veintiuno Editores

Palti, Elías (2007b), Lugares y no lugares de las ideas en América Latina, en: Palti (2007a), 259-308

Palti, Elí́as José (2008), La invención de una legitimidad. Razón y retórica en el pensamiento mexicano del siglo XIX (Un estudio sobre las formas del discurso político), Primera edición argentina, Mexico: Fondo de cultura económica

Parsons, Talcott (1985), On Institution and Social Evolution. Selected Writings, Ed. León H. Mayhew, Chicago: The University of Chicago Press

Scherner, Karl Otto, Christoph Bergfeld (1993), Modernisierung des Handelsrechts im 19. Jahrhundert, Heidelberg: Recht und Wirtschaft

Schwarz, Roberto (1977), As ideias fora do lugar, en: Schwarz, Roberto, Ao vencedor as batatas. Forma literária e processo social nos inícios do romance brasileiro, São Paulo: Librária Duas Cidades, 11-31 


\section{Le parole della modernizzazione latinoamericana}

Centro, periferia, individuo e ordine

\section{Modernizzazione e classificazione dei diritti nazionali: il diritto latinoamericano come periferia}

Secondo le definizioni più diffuse, la modernizzazione è il processo attraverso cui una società acquisisce le caratteristiche ritenute proprie della modernità: in particolare caratteristiche di ordine economico e politico, concernenti rispettivamente l'affermazione e lo sviluppo del capitalismo e della democrazia. ${ }^{1}$ A questo modo di intendere la modernizzazione corrispondono le definizioni, altrettanto diffuse, del diritto occidentale come fenomeno sociale autonomo, ${ }^{2}$ ma pur sempre fondato «sui principi politici e sociali della democrazia liberale e su strutture economiche capitaliste». ${ }^{3}$

E' noto che il nucleo del diritto occidentale è rappresentato dalle esperienze di common law e civil law, originarie rispettivamente dell'Europa continentale e dell'Inghilterra. Altrettanto noto è il carattere tendenzialmente globale della modernizzazione, a cui corrisponde la circolazione dei modelli giuridici tipici di quelle esperienze, punto di riferimento per classificare numerosi ordinamenti nazionali a livello planetario, inclusi quelli dell'area latinoamericana. Fin dal Congresso di diritto comparato in occasione dell'Esposizione universale tenutasi a Parigi nel 1900, evento considerato l'atto di nascita della materia, si afferma infatti che tra $\mathrm{i}$ «sistemi di diritto originale» si annovera il «gruppo latino, comprendente la Francia, il Belgio, l'Italia, la Spagna, il Portogallo, la Romania e le Repubbliche latine dell'America centrale e meridionale». ${ }^{4}$

1 Martinelli (2002) 9.

2 Berman (1983) 7 ss.

3 David (1950) 224.

4 Esmein (1900) 495. 
Seppure nel corso dei decenni si è riconosciuta l'originalità del diritto latinoamericano, ${ }^{5}$ o almeno di alcune soluzioni considerate un suo tratto tipico, come in particolare la codificazione del diritto civile brasiliano del $1917,{ }^{6}$ è resistita a lungo l'idea per cui il gruppo latino è tale per l'adozione di codificazioni del diritto civile più o meno modellate sul testo francese. ${ }^{7} \mathrm{Si}$ è in tal modo costruita ad arte una sorta di gerarchia tra ordinamenti nazionali ricondotti al medesimo gruppo, strutturata a partire dalle dinamiche relative alla circolazione del modello reputato originale. Se infatti i Paesi dell'America centrale e meridionale si sono ispirati alla codificazione francese, è perché lo stesso hanno fatto in particolare la Spagna e il Portogallo, ovvero i principali colonizzatori di quella porzione di mondo. E ciò equivale a dire che, se la Francia è collocata al centro dell'esperienza giuridica occidentale, i Paesi latinoamericani ne rappresentano la periferia.

Questo modo di concepire l'area latinoamericana e il suo diritto ricorre soprattutto nelle ricostruzioni più risalenti. Tuttavia, esso appare ancora sufficientemente diffuso, mostrandosi come il portato in buona sostanza ineliminabile della classificazione dei diritti nazionali in famiglie giuridiche $o$ Rechtskreise: ${ }^{\mathbf{8}}$ anche di recente la letteratura giuscomparatistica, pur talvolta avvertendo circa le banalizzazioni che ciò comporta, ${ }^{9}$ si limita a menzionare il diritto privato latinoamericano come periferia della famiglia di civil law. ${ }^{\mathbf{1 0}}$ E magari spiega così, muovendo cioè dalla sua «origine legale», la minore capacità di quel diritto di produrre benessere materiale: questo affermano ad esempio i fautori della New comparative economics nell'ambito dell'approccio di Law and finance. ${ }^{\mathbf{1 1}}$

Certo, non mancano coloro i quali sono disposti a riconoscere l'autonomia del diritto latinoamericano, tuttavia solo nella misura in cui esso coincide con l'esperienza giuridica indigena, ${ }^{\mathbf{1 2}}$ trascurando la quale permane il rapporto di dipendenza dal diritto occidentale, il cui presidio finisce per divenire il motivo ispiratore della classificazione proposta.

5 Citazioni in: Pargendler (2012) 5 ss. e Rosti (2007) 3 ss.

6 Sarfatti (1933) 78.

7 Arminjon, Nolde, Wolff (1950) 50.

8 Zweigert, Kötz (1996) 62 ss. Al proposito Dölemeyer (2010).

9 Per tutti Legeais (2004) 160.

10 Ad es. Zweigert, Kötz (1996) 112 ss. e Fromont (1998) 6.

11 Djankov et al. (2003) 595 ss.

12 Ad es. David, Jauffret-Spinosi (2002) 56.

12 Alessandro Somma 
Tutto ciò riguarda i comparatisti cultori del diritto privato, naturalmente inclini a collocare il codice civile al centro delle loro classificazioni. Chi invece utilizza il punto di vista del diritto costituzionale, segnala che il diritto latinoamericano è debitore nei confronti delle elaborazioni provenienti dalla famiglia di common law, per affermare in particolare che esso costituisce una periferia del centro statunitense: ${ }^{13}$ è da quest'ultimo che si sono in particolare mutuati il federalismo e il presidenzialismo. ${ }^{\mathbf{1 4}}$

Seppure ancora resistenti, le classificazioni dei diritti nazionali per famiglie giuridiche vengono da tempo criticate. Sono infatti oramai ricorrenti i contributi in cui le si reputa meri espedienti didattici, necessariamente produttivi di semplificazioni prive di valore scientifico. ${ }^{15}$ Lo si afferma con riferimento alle classificazioni più tradizionali, a partire da quella ricavata dalla distinzione tra ordinamenti di civil law e common law, ${ }^{\mathbf{1 6}}$ che si propone di sostituire con altre, concernenti in particolare le modalità di controllo o incentivazione sociale. ${ }^{17}$ In tal modo si osservano, accanto alle modalità di «governo» della società e dunque vicende relative alle «macro-scelte», anche il funzionamento della «organizzazione sociale», ovvero la «gestione della generalità dei rapporti sociali considerati nella loro quotidianità», valorizzando i quali si producono classificazioni che non peccano di «eurocentrismo». ${ }^{18}$

Così l'area latinoamericana perde in effetti il carattere di periferia rispetto all'area europea continentale, quest'ultima ricondotta ai sistemi a «egemonia professionale», e tuttavia finisce per essere identificata con caratteristiche che la pongono comunque in posizione subordinata rispetto al Vecchio mondo. Ciò traspare dall'inclusione delle esperienze latinoamericane tra gli ordinamenti a «egemonia politica», in quanto la loro posizione di «Paesi in transizione e in via di sviluppo» non consente di annoverarli tra quelli che hanno realizzato quanto si reputa un carattere fondativo dei sistemi a egemonia professionale: il divorzio tra circuito del diritto e circuito della politica. ${ }^{19} \mathrm{E}$ ciò equivale a dire che, se l'area latinoamericana non costituisce

13 Ad es. Schlesinger et al. (1998) 291.

14 Mezzetti (2009) 471 s. e 482.

15 Cfr. Agostini (1988) 25 e Kötz (1998) 493 ss.

16 Gordley (2003) 498 ss.

17 Mattei (1997) $5 \mathrm{ss}$.

18 Mattei, Monateri (1997) $63 \mathrm{s.}$

19 Mattei (1997) 28. 
la periferia di un modello, essa rappresenta però il centro di un diverso modello reputato complessivamente periferico, tale in quanto arretrato rispetto alle esperienze occidentali.

Per quanto avanzate, evidentemente le classificazioni dei diritti nazionali in quanto tali non sono idonee a restituire fedeli descrizioni della realtà. Esse esprimono punti di vista indissolubilmente legati alle propensioni e ai limiti culturali dei loro autori, i quali ricorrono a metodi di analisi che appaiono individuati ad arte per produrre il risultato voluto. ${ }^{20}$ Metodi con cui esaltare le similitudini tra esperienze giuridiche collocate in tempi e spazi differenti, e sacrificare nel contempo le differenze riconducibili a una più attenta considerazione del contesto entro cui si sono sviluppate. ${ }^{21}$

Nel corso di questo lavoro metteremo in luce i caratteri delle classificazioni utilizzate per produrre una visione del diritto latinoamericano come periferia del diritto occidentale, muovendo nel merito dalle modalità attraverso cui viene spiegata la mutazione giuridica. Valuteremo poi la possibilità di produrre classificazioni capaci invece di valorizzare i caratteri distintivi dell'esperienza latinoamericana, considerando il contributo fornito da chi esalta il carattere plurale della modernizzazione. Tenteremo cioè di tracciare i caratteri della modernizzazione latinoamericana come relativa allo sviluppo del capitalismo e della democrazia in termini autonomi, oltre che differenti rispetto a quelli che hanno caratterizzato l'area occidentale. $\mathrm{E}$ in tale prospettiva non potremo non accennare all'esperienza giuridica indigena come tratto peculiare dell'area latinoamericana che, richiamandosi alla tradizione, si presenta formalmente come esperienza non descrivibile a partire dai caratteri della modernizzazione.

Infine utilizzeremo i tratti identificativi della modernizzazione latinoamericana per compiere l'operazione opposta a quella che conduce a discuterne come di una vicenda autonoma: per individuare equivalenze funzionali tra quest'ultima e la modernizzazione così come essa si è sviluppata in area europea e statunitense. Potremo così mettere in luce la fragilità del nesso tra democrazia e capitalismo come tratto distintivo della modernizzazione tout court, e dunque del diritto occidentale come garante di quel nesso.

20 Legrand (1999) $15 \mathrm{~s}$.

21 Cfr. Sомma (2005c) 42 ss.

14 Alessandro Somma 
2 Modernizzazione e mutazione giuridica tra evoluzionismo e diffusionismo

I comparatisti sono soliti discutere della classificazione dei diritti nazionali in connessione con il tema della mutazione giuridica, ovvero delle modalità attraverso cui quei diritti cambiano nel corso del tempo. E' questa una prospettiva particolarmente rilevante ai nostri fini, giacché la collocazione del diritto latinoamericano alla periferia del diritto occidentale si fonda sulla circostanza che esso cambia in senso capitalistico e democratico, seppure secondo tempi e modi tipici appunto di una periferia.

In un primo tempo i comparatisti hanno spiegato la mutazione giuridica alla luce di paradigmi evoluzionisti, per cui il diritto è naturalmente destinato a svilupparsi in modo unilineare, secondo uno schema nel quale il progresso rappresenta un valore universale e la tradizione un indizio di arretratezza. Il tutto nel solco di credenze diffuse tra i teorici della modernizzazione, i quali hanno sovente identificato il progresso con lo sviluppo del capitalismo e della democrazia, seppure con una particolare enfasi sul primo dei due termini. ${ }^{22}$

Evoluzionista è in particolare il considerare il diritto del capitalismo e della democrazia come diritto dei popoli civilizzati e dunque come diritto moderno tout court, contrapposto a un diritto dei popoli arretrati inteso come diritto tradizionale, o comunque deprecabilmente rivolto al passato. ${ }^{23}$ E' questo uno schema espositivo tipico della letteratura comparatistica risalente, legata a un'impostazione che almeno in parte è penetrata persino nello Statuto della Corte internazionale di giustizia. Come è noto quest'ultimo menziona infatti, tra le fonti del diritto internazionale, $\mathrm{i}$ «principi generali del diritto riconosciuti dalle nazioni civili»: espressione con cui si è voluto alludere a «una opinione del giusto ritenuta universale». ${ }^{24}$

Si ricava da quanto affermato nel corso del menzionato Congresso parigino di diritto comparato del 1900, che l'approccio evoluzionista è connaturale alla classificazione dei diritti nazionali per famiglie giuridiche, o quantomeno che essa si è sviluppata a partire da quell'approccio. Allora si disse che la classificazione per famiglie risultava dalla combinazione di comparazione e sociologia giuridica, la seconda votata all'individuazione delle

22 Citazioni in Martinelli (2002) $47 \mathrm{ss.}$

23 Arminjon, Nolde, Wolff (1950) 47.

24 Tripiccione (1961) 350. 
«leggi di psicologia sociale», ${ }^{25}$ ovvero delle regole concernenti il funzionamento della società intesa come entità distinta dagli individui che la compongono. In tale prospettiva si muoveva da un'idea di evidente matrice positivista, ovvero che l'evoluzione del diritto fosse «una concatenazione regolata, uniforme e inevitabile di fasi successive». Di questa il diritto comparato doveva «tracciare la traiettoria», ricorrendo nel merito alla «elaborazione di classificazioni naturali e quindi razionali», proprio come quelle concernenti l'individuazione di «famiglie di diritti». Il che, se per un verso non portava ad accreditare l'esistenza di «un solo diritto naturale», per un altro apriva la strada alla ricerca del più «avanzato» tra i diversi «tipi giuridici», in quanto tale inevitabilmente destinato a essere imitato. ${ }^{\mathbf{2 6}}$

Per quanto viziate, queste ricostruzioni aprono la strada al paradigma diffusionista come alternativo a quello evoluzionista nella spiegazione della mutazione giuridica, finalmente ritenuta un effetto del contatto tra culture piuttosto che dell'innovazione originale, seppure naturalmente o inesorabilmente orientata in modo unidirezionale. E proprio il diffusionismo ha riscosso un certo successo tra i comparatisti, come documenta la circostanza che la menzionata nozione di famiglia giuridica deriva da un concetto diffusionista come quello di famiglia culturale o Kulturkreis: concetto elaborato sul finire dell'Ottocento per descrivere il contesto spaziale entro cui si diffonde un certo tratto culturale, o entro cui si ritrovano manifestazioni particolari di modelli comuni a una pluralità di contesti. ${ }^{27}$

Peraltro, come abbiamo visto, non basta invocare l'imitazione come motore della mutazione giuridica per evitare le forzature riconducibili all'adozione del credo evoluzionista. In ambito diffusionista si afferma infatti che la creazione di un modello originale costituisce un fatto raro, ${ }^{28}$ alimentando così visioni della mutazione giuridica come essenzialmente dovuta alla mera recezione del modello esportato senza apporto creativo da parte dell'ordinamento importatore. Si finisce cioè per costringere la mutazione giuridica entro un rapporto gerarchico tra un centro votato all'esportazione di modelli, e una periferia destinata alla loro importazione: è infatti questo lo

25 TARDE (1900) 529.

26 TARDE (1900) $531 \mathrm{~s}$.

27 Frobenius (1897) 1 ss. e Frobenius (1898) 1 ss.

28 Per tutti Gambaro, Sacco (1996) $31 \mathrm{s.}$ 
schema classico utilizzato per descrivere le modalità con cui, in area latinoamericana, il diritto cambia.

Ma non è tutto. In letteratura si suole dire che la scelta del modello da imitare dipende da «una qualità che non sappiamo come chiamare, se non con il nome prestigio». ${ }^{29}$ Una qualità oltretutto valutata autonomamente dalla classe dei cultori del diritto dell'ordinamento imitatore, i quali operano pertanto secondo criteri di ordine tecnico, essendo tutt'al più condizionati nella scelta dall'effettiva accessibilità di quanto si reputa prestigioso: ${ }^{30}$ in particolare dalla conoscenza o meno della lingua del modello da importare. ${ }^{31}$ Il diffusionismo occulta così la recezione imposta di modelli, ${ }^{32}$ dettata da rapporti di potere come quelli originati dal colonialismo, che sono stati il principale motore della circolazione del diritto occidentale. Si finisce così, inevitabilmente, per alimentare discorsi di matrice evoluzionista, espliciti nel rilievo per cui il prestigio di un modello deriva dal suo essersi accreditato come capace di edificare una «società più avanzata in termini di civiltà giuridica». ${ }^{33}$

Proprio a partire da questo schema, tra gli anni cinquanta e sessanta del secolo scorso, si è sviluppato il movimento di Law and development, intenzionato ad approfondire la relazione tra mutazione giuridica e sviluppo economico anche e soprattutto in area latinoamericana. Ebbene, in quell'ambito venne fin da subito tracciata la distinzione tra Paesi «avanzati» e Paesi «inseguitori», i primi zelanti produttori e i secondi meri assimilatori di modelli giuridici occidentali, ritenuti la punta avanzata in termini di progresso. ${ }^{34}$ E fin da subito fu evidente che i modelli da sponsorizzare erano quelli mutuati dal common law statunitense, punto di riferimento per quanto è stata ritenuta, più che una forma di assistenza legale, un'operazione di vero $\mathrm{e}$ proprio «imperialismo giuridico». ${ }^{35}$

Non si intende qui rifiutare l'idea di una occidentalizzazione dell'area latinoamericana, ${ }^{36}$ speculare rispetto al carattere tendenzialmente globale dei

29 SACCO (1992) 147.

30 Watson (2001) 263.

31 Cfr. Sомma (2001) 258 ss.

32 Ad es. Rheinstein (1987) 125.

33 Grande (2000) 44 s. e 149.

34 Cfr. Benedix (1964) 413.

35 Cfr. Gardner (1980) part. 27 ss.

36 Per tutti Carmagnani (2003) 302 ss. 
processi di modernizzazione, che non costituisce di per sé l'indizio di un approccio alla circolazione dei modelli come quello appena criticato. Lo diverrebbe, tuttavia, se si volesse con ciò affermare che, magari in ritardo sui tempi della modernizzazione occidentale, l'area latinoamericana ha recepito passivamente modelli prodotti altrove: come in modo più o meno esplicito fanno pensare le tradizionali classificazioni di diritti nazionali in famiglie giuridiche. Si trascura così che l'imitazione implica innanzi tutto una selezione necessariamente arbitraria di quanto si reputa opportuno imitare. ${ }^{37} \mathrm{E}$ che al processo di imitazione dei modelli è coessenziale una loro modifica e integrazione, «una reinvenzione a livello periferico del diritto del centro». ${ }^{\mathbf{3 8}}$ Soprattutto per questo motivo il ruolo dell'America latina è stato ed è di «attore attivo nel processo di occidentalizzazione», ${ }^{39}$ che dunque, a rigore, può essere definito tale solo se si accetta di valutarlo oltre il limitato schema del rapporto tra centro e periferia.

Se si vuole superare questo schema, se cioè si muove dal carattere essenzialmente creativo dell'imitazione di modelli giuridici, e a monte dalla complessità dei processi di diffusione del diritto ${ }^{40}$ allora si deve evitare l'utilizzo di espressioni che preludono invece a un ruolo passivo del soggetto imitatore.

Una di queste è senza dubbio l'espressione «trapianto giuridico». ${ }^{41} \mathrm{Se}$ condo i critici più accesi di questa espressione, essa indica una vicenda irrealistica, in quanto i modelli giuridici possiedono un patrimonio ermeneutico che non può essere importato senza alterazioni: «il significato non si presta a essere trapiantato». ${ }^{42}$ Similmente si precisa che di trapianto potrebbe parlarsi solo nelle ipotesi in cui un gruppo umano si sposta portando con sé il proprio diritto: circostanza forse concepibile unicamente nelle esperienze in cui si ammette l'operare del principio di personalità del diritto, o nelle ipotesi in cui lo spostamento avviene su una porzione di territorio non soggetta ad alcuna autorità costituita. ${ }^{43}$ In tutte le restanti ipotesi non di trapianti si dovrebbe parlare, i quali sovente presuppongono la morte

37 Cfr. Lopez-Medina (2004).

38 Marini (2011) 168.

39 Carmagnani (2003) ix.

40 Twining (2005) 203 ss.

41 Watson (1993).

42 Legrand (2001) 55 ss.

43 Rheinstein (1987) 126. 
dell'organismo donante, bensì di «innesti» che si concretano nella «fusione delle vite di due organismi», il donante e il ricevente, $o$ in alternativa nella «sovrapposizione dell'uno all'altro». ${ }^{\mathbf{4 4}}$

Alla mera sovrapposizione sembra fare riferimento la letteratura antropologica in sede di analisi del pluralismo giuridico, determinato fra l'altro dalle ipotesi in cui il modello importato conserva la sua identità all'interno del sistema imitatore sotto forma di «strato». Questo si ricaverebbe specialmente dallo studio degli ordinamenti interessati da mutazioni notevoli avvenute nell'arco di periodi relativamente brevi, come quelle tipicamente imposte dalla colonizzazione. In simili casi si trovano, a fianco di uno strato tradizionale consuetudinario caratteristico delle società a potere diffuso, uno strato coloniale costituito dal diritto dei paesi occidentali occupanti, quindi uno strato postcoloniale anch'esso modellato sul diritto dei Paesi occidentali. ${ }^{45}$

E' peraltro lecito ritenere che la mera sovrapposizione di modelli autoctoni e modelli importati finisca in un modo o nell'altro per produrre fusioni o almeno confusioni, le quali finiscono a loro volta per mettere in luce l'assenza di una corrispondenza biunivoca tra diritto e società, riconducibile del resto a paradigmi metodologici oramai superati. Se infatti i modelli giuridici da imitare sono selezionati a partire da una loro associazione con assetti di ordine politico o economico, sono innanzi tutto questi ultimi a non essere suscettibili di riprodursi in modo fedele. E' anche questo un modo per ribadire che la circolazione dei modelli non avviene nell'ambito di un rapporto tra centro e periferia della produzione giuridica: un modo per denunciare la parzialità di un simile punto di vista, insieme con il carattere ideologico dei relativi risvolti.

\section{Il rapporto tra diritto e società: dalle famiglie} alle tradizioni giuridiche

Muovendo dal punto di vista della mutazione giuridica, abbiamo ricavato ulteriori elementi idonei a rafforzare il convincimento che le classificazioni dei diritti nazionali sono tradizionalmente pensate in funzione di un risultato ben preciso: documentare la superiorità del diritto occidentale come motore

44 Feldman (1997) $220 \mathrm{~s}$.

$45 \mathrm{Al}$ proposito ad es. Mattei, Monateri (1997) 148 ss. 
della modernizzazione, e presentare così il suo recepimento come prospettiva desiderabile, e insieme inevitabile, a livello planetario. Un simile proposito ispira la riconduzione dell'esperienza giuridica latinoamericana alla famiglia di civil law, in quanto la prima si sarebbe sviluppata a partire da un'imitazione di modelli elaborati in area europea continentale, seppure combinati con modelli di provenienza statunitense. Il tutto in virtù del prestigio dei modelli imitati, la cui circolazione viene documentata esaltando ad arte i caratteri del modello imitatore che lo accreditano come periferia della mutazione giuridica, e occultando invece quelle da cui ricavare la sua originalità.

Per svelare il carattere ideologico di una simile operazione, occorre insistere sull'assenza di un nesso biunivoco tra diritto e società, aspetto cui implicitamente hanno fatto riferimento i fautori del movimento di Law and development, soprattutto quelli impegnati a riflettere sulle cause del suo fallimento. Quel movimento traeva forza dalla credenza per cui la mera imitazione di modelli giuridici dei Paesi «avanzati» da parte dei Paesi «inseguitori» avrebbe innescato processi di modernizzazione, e dunque di sviluppo in senso capitalista e democratico. Credenza poi criticata, almeno nella misura in cui non veniva corretta dalla considerazione per le fonti non formali di produzione del diritto ${ }^{46}$ e dalla cura per la formazione degli operatori dell'ordinamento importatore. ${ }^{47}$

Questi rilievi non erano però capaci di rifondare in modo corretto il rapporto tra diritto e società, se è vero che erano seguiti dalla precisazione di sapore evoluzionista per cui, se per un verso il mutamento giuridico segue di norma il mutamento sociale, per un altro esso richiede «una professione legale incline all'attivismo riformatore». ${ }^{48}$ Caratteristica, quest'ultima, considerata tipica prevalentemente, se non unicamente, del contesto statunitense, ${ }^{49}$ a dimostrazione di quanto il movimento di Law and development sia il prodotto di una ideologia ben precisa: la «fede incondizionata nella validità del sistema giuridico statunitense». ${ }^{50}$

Più utile ai nostri fini è la letteratura che, seppure da un punto di vista diffusionista classico, discorre del valore simbolico dei modelli giuridici

46 Trubek, Galanter (1974) 1062 ss.

47 Cfr. Burg (1977) 492 ss.

48 Friedman (1978) 453.

49 Merryman (1977) 457 ss.

50 EsQuirol (2011) 252. 
importati, ovvero della circolazione non del «modello in sé», bensì della «immagine di tipo politico-filosofico a esso comunemente associata». ${ }^{\mathbf{5 1}}$ Utile in quanto valorizza la circostanza che i modelli di origine francese erano ricercati anche e soprattutto per la carica rivoluzionaria che esprimevano all'epoca in cui l'area latinoamericana, liberatasi dalla dominazione coloniale, intendeva dotarsi di un ordinamento a misura di individui liberi e indipendenti: ${ }^{52}$ finalità che, sul versante del diritto costituzionale, rendeva evidentemente interessanti anche i modelli di origine statunitense.

Anche da ciò trae conferma l'idea che occorre considerare i modelli giuridici circolanti come pratiche discorsive che subiscono notevoli trasformazioni lungo il loro percorso, ${ }^{53}$ esprimendo indefinite e indefinibili "possibilità e impossibilità enunciative». ${ }^{54} \mathrm{Di}$ qui la necessità di operare una dissociazione tra tecniche giuridiche e valori alla cui promozione esse sono asservite, per poi concentrare l'attenzione su questi ultimi. ${ }^{55}$ Il tutto volendo precisare che, se di impossibilità dei trapianti si vuole parlare, è per affermare che la circolazione dei modelli interessa a ben vedere i valori di cui quei modelli costituiscono il veicolo, e che pertanto uno studio dei termini in cui essa si manifesta deve interessare vicende situate oltre i confini del discorso attorno al diritto in senso stretto.

Se così stanno le cose, occorre superare quanto di norma sottintende il riferimento al concetto di sistema o ordinamento cui prelude la locuzione «famiglie giuridiche», in quanto concetto che rinvia a una «sistematica chiusa e delimitata», tipica della "ricerca obbiettiva tanto cara allo scientismo positivista». ${ }^{\mathbf{5 6}}$ Proprio per ovviare a questi vizi si è proposto il ricorso alla «tradizione giuridica» come punto di riferimento per le classificazioni, ricorso particolarmente diffuso nelle trattazioni confezionate dai common lawyer. ${ }^{57}$

Per questi ultimi, il riferimento alla tradizione costituisce il riverbero di un modo di rapportarsi al mondo del diritto ricalcato sulle caratteristiche dell'ordinamento di provenienza: un ordinamento in cui il potere politico non ha alimentato una retorica sulla cesura tra l'epoca in cui la convivenza tra

51 Grande (2000) $110 \mathrm{~s}$.

52 Mirow (2009) $285 \mathrm{~s}$.

53 Per tutti Rouland (1998) 449 ss.

54 Foucault (1999) 169 ss.

55 Sомma (2005c) 64 ss.

56 Orestano (1987) 352.

57 Giaro (2003) 343 ss. 
i consociati era informata a un sistema di norme di diritto comune, e l'epoca in cui essa è stata poi plasmata dalla volontà di un principe o di un parlamento onnipotente. ${ }^{58} \mathrm{Di}$ qui la contrapposizione fra tradizione giuridica e diritto nazionale, quindi il rifiuto del riferimento alla illuministica «autorità normativa delle fonti formali del diritto» cui quest'ultimo rinvia. ${ }^{59}$

I riferimenti alla tradizione giuridica segnalano così l'intento di confezionare scritti di impostazione antipositivista, o comunque scettici verso l'approccio di chi vede nel diritto un efficace strumento di ingegneria sociale: intento in linea con la valorizzazione del diritto come pratica discorsiva, talvolta espresso attraverso l'utilizzo dell'espressione "cultura giuridica». ${ }^{\mathbf{6}}$ Peraltro la semplice manifestazione di un simile proposito nulla dice circa la sua effettiva realizzazione, giacché la combinazione tra storia e comparazione ha costituito il fondamento per le più disparate opzioni culturali: da quelle volte a individuare le costanti universalistiche del fenomeno diritto, a quelle pensate per sottolinearne la contingenza, passando per una contraddittoria combinazione dei due approcci. ${ }^{\mathbf{6 1}}$

Ci sono insomma molti riscontri circa il fatto che il ricorso alla «tradizione giuridica» non indichi un significativo scarto rispetto all'impiego di «famiglia giuridica», ${ }^{62}$ primi fra tutti quelli che si ricavano dal riferimento al diritto occidentale come «tradizione giuridica occidentale». Quest'ultima viene infatti utilizzata per sottolineare come le esperienze di common law e civil law siano accomunate dall'aver realizzato il divorzio tra il fenomeno diritto e i restanti fenomeni sociali. ${ }^{63}$ L'uso di «tradizione giuridica» alimenta cioè operazioni culturali in cui non si valorizza a sufficienza la dimensione in senso ampio storica del diritto, $o$ in alternativa operazioni in cui quest'ultima viene invocata al solo fine di accreditare il diritto come forma di controllo sociale radicata nei secoli e dunque prestigiosa.

Senza contare poi che la tradizione, in quanto parte essenziale della memoria sociale, assume forma attraverso un processo di selezione di informazioni sul passato, pratica arbitraria per definizione perché non riducibile

58 Ad es. Barton et al. (1983) 1 ss.

59 GLENN (2000) xxi.

60 Cfr. Nelken (2007) 111 ss.

61 Citazioni in Somma (2005a) 169 ss.

62 Cfr. de Cruz (1999) 33 ss.

63 Ad es. Gambaro, Sacco (1996) 41 ss.

22 Alessandro Somma 
alla loro mera trasmissione. ${ }^{64}$ Proprio questa circostanza viene esemplarmente chiarita da chi discorre di «tradizioni inventate», per indicare le narrazioni che stabiliscono momenti di continuità «in larga misura fittizia» con un «passato storico opportunamente selezionato». ${ }^{65}$

Volendo verificare i risvolti effettivi di un ricorso alle tradizioni giuridiche a fini classificatori, possiamo consultare quanto un noto autore statunitense ha scritto in materia di «tradizione di civil law», per «quei lettori desiderosi di apprendere che cosa unisce i sistemi giuridici dell'Europa occidentale e dell'America latina». Nel merito rileva la premessa dedicata alla Francia e alla Germania, considerate il centro della tradizione di civil law, ma anche esperienze «atipiche» nell'ambito di quella tradizione. Se infatti i due Paesi occupano al suo interno una posizione di «leadership intellettuale», non si sono condizionati a vicenda e dunque non possiedono quanto accomuna gli ordinamenti di civil law: l'essersi sviluppati a partire dalla «recezione e fusione» di entrambe le influenze francese e tedesca. ${ }^{\mathbf{6 6}}$

Una simile premessa, seppure conduce a qualificare come atipici gli ordinamenti posti al centro della tradizione di civil law, non mette in discussione il rapporto gerarchico tra il centro europeo e la periferia latinoamericana, e con esso il peso attribuito ai caratteri tradizionalmente evocati per sostenere quel rapporto: primo fra tutti il ruolo della codificazione civile "come espressione di un'ideologia», pur mitigata nel tempo dalle innovazioni legate al costituzionalismo, che si sviluppa in area europea, per essere poi recepita dal continente sudamericano. ${ }^{67}$ Schemi assimilabili si ricavano da altra letteratura di common law, dove si mette in luce la valenza simbolica della codificazione francese, quindi la sua forza attrattiva in quanto testo capace di sintonizzarsi con i sentimenti di chi si è da poco liberato dai propri colonizzatori. $^{68}$

Se dunque si eccettuano contributi come quello a cui faremo tra breve riferimento, il ricorso al concetto di tradizione giuridica nella classificazione dell'esperienza latinoamericana non ha prodotto risultati significativamente alternativi a quelli ricavabili dalle tradizionali classificazioni per famiglie

64 Cavalli (1998) $649 \mathrm{~s}$.

65 Hobsbawm (2002) $3 \mathrm{~s}$.

66 Merryman (1973) $3 \mathrm{~s}$.

67 Merryman (1973) 40 ss.

68 Glendon, Gordon, Carozza (1999) 45. 
giuridiche. Chi discute di tradizione giuridica, se anche enuncia l'intento di valorizzare tematiche diverse da quelle prese in considerazione dal positivismo, non giunge a classificazioni molto dissimili da quelle cui perviene chi ricorre al concetto di famiglia giuridica. Si possono cioè ampliare gli orizzonti della classificazione, includendovi dati di natura in senso lato culturale, e tuttavia il proposito di produrre narrazioni attorno ai «grandi sistemi» finisce per sacrificare la specificità degli ordinamenti che in quelle narrazioni rivestono un ruolo tipicamente subordinato. ${ }^{69}$

Il tutto mentre l'attrattiva di un punto di riferimento per l'importazione di modelli in area latinoamericana, il codice civile francese come codificazione per antonomasia, ha da tempo perso lo smalto posseduto sino a diversi decenni or sono, e con ciò il valore simbolico attribuito alla sua circolazione. ${ }^{70}$ Già nel corso delle celebrazioni per il primo centenario dell'articolato, si ammetteva infatti che il suo prestigio si stava rapidamente contraendo. ${ }^{71}$ Tanto che negli anni sessanta del Novecento non pochi autori discorrevano di un «regresso quasi generale» del modello codicistico francese, in alcuni casi tale «da far apparire grottesche le classificazioni tradizionali». ${ }^{72}$

Da ciò non deriva però l'impulso per una diversa considerazione dell'area latinoamericana, per il superamento della tradizionale prospettiva per cui essa costituisce la periferia del centro europeo. Deriva invece, in linea con i fondamenti di quella prospettiva, l'individuazione dei diritti occidentali che avevano sostituito il francese nel ruolo di centro di una produzione giuridica: tra gli altri il diritto italiano, o lo svizzero, o ancora il common law ${ }^{73}$ In alternativa si assiste a uno spostamento dell'asse attorno a cui far ruotare la comune matrice del diritto occidentale e latinoamericano, secondo schemi incapaci di determinare un superamento dell'approccio etnocentrico. Proprio questo accade in particolare nei contributi storico-comparatistici in cui si esalta il diritto romano come principale elemento identificativo dell'esperienza di civil law. ${ }^{74}$

69 VANDERLINDEN (1995) 375.

70 Cfr. Somma (2006).

71 V. i contributi in Société D’ÉTUdes LÉGISLATIVES (1904) 583 ss.

72 Grisoli (1962) $123 \mathrm{~s}$.

73 Abert (1978) 53.

74 SCHipani (1981).

$24 \quad$ Alessandro Somma 
I comparatisti hanno insomma confezionato classificazioni di ordinamenti nazionali che riconducono al diritto occidentale quelli in qualche modo orientati verso la costruzione di società capitaliste e democratiche. Essi hanno così costruito narrazioni al servizio del carattere tendenzialmente globale dei processi di modernizzazione, che elevano l'area europea e statunitense a centro produttivo di modelli di convivenza sociale, destinati a essere imitati dalle periferie del pianeta. In tale prospettiva il diritto latinoamericano ha finito per trovarsi «nel mezzo tra il mondo occidentale normalizzato ed egemonico e quello orientale esotico e subalterno». Ha finito cioè per essere inteso come «riproduzione mimetica dell'Occidente avanzato», quindi come «ombra alquanto pallida del diritto occidentale», o peggio ancora come «parziale e povera lettura di autori che possono essere padroneggiati solo in ricchi contesti interpretativi». ${ }^{75}$

Vedremo fra breve come questo schema possa essere messo in crisi approdando a letture pluraliste della modernizzazione, utilizzabili ad esempio per esaltare le peculiarità dell'area latinoamericana, superando il rapporto tra centro e periferia, e dunque prescindendo dalla sua carica ideologica. Prima di fare questo, ci concentreremo sui tratti distintivi del diritto latinoamericano ricavabili dalla letteratura che lo pone a confronto con il diritto occidentale in senso stretto.

In via preliminare possiamo osservare che, in un certo senso, la ricostruzione di un'identità latinoamericana traspare anche dalle classificazioni tradizionali. Si tratta però di un'identità negativa, ricavata dall'elencazione dalle mancanze che si suppone abbiano caratterizzato il processo di imitazione del modello occidentale, secondo uno schema che appare mutuato dalla teoria sulle fasi e le soglie critiche della modernizzazione. ${ }^{76}$ Il tutto sul presupposto, esplicitato in termini così crudi al principio del secolo scorso, che «il selvaggio non copia nulla senza snaturarlo per goffaggine, esattamente come i bambini sono soliti disegnare». ${ }^{77}$

Certo, negli esempi cui faremo ora riferimento, i termini sono meno crudi, ma non per questo meno indicativi di un'attitudine etnocentrica

75 López Medina (2011) 198 s. e 213.

76 Su cui ad es. Martinelli (2002) 47 ss.

77 TARDE (1900) 536. 
nell'analisi della mutazione giuridica, troppo spesso tesa verso «l'esoticizzazione dell'altro». ${ }^{78}$ Questo si ricava dall'opinione secondo cui la qualità dei giudici dell'area latinoamericana sarebbe particolarmente bassa a fronte della scarsa attrattiva della professione, così come dalla constatazione che la «purezza della scienza giuridica», particolarmente resistente in quell'area, avrebbe reso il diritto uno strumento inadatto a «soddisfare i bisogni della società». ${ }^{79}$ Lo stesso dicasi con riferimento a chi, più recentemente, svaluta il recepimento latinoamericano del presidenzialismo statunitense, ritenendolo difettoso per essere stato realizzato in forme incapaci di produrre un efficace meccanismo di checks and balances. ${ }^{\mathbf{8 0}}$

Queste letture stupiscono, in quanto considerano tipici della periferia latinoamericana difetti che pure caratterizzano il centro del modello occidentale. La giuscomparatistica tradizionale ammette infatti che le deviazioni rispetto alla tripartizione dei poteri possono essere viste come un'eredità della tradizione autoritaria spagnola. ${ }^{\mathbf{8 1}}$ Mentre la letteratura più consapevole dei rischi di etnocentrismo segnala influenze europee sul caudillismo, da ritenere anche una versione latinoamericana del cesarismo europeo, considerata un retaggio del modello napoleonico, oltre che un riscontro di quanto abbiano attecchito dottrine come quelle sviluppate a partire dal pensiero di Auguste Comte. ${ }^{82}$ E difatti proprio il positivismo comtiano, nella letteratura europea come in quella latinoamericana, era invocato in combinazione con visioni organicistiche ed evoluzioniste della società, o comunque a sostegno del proposito di produrre per l'individuo costruzioni idonee a valorizzare il profilo del suo scioglimento in un ordine votato al progresso. ${ }^{\mathbf{8 3}}$

Il tutto mentre, con riferimento alla nascita del costituzionalismo latinoamericano come costituzionalismo impregnato di nazionalismo interclassi$\mathrm{sta}^{84}$ è bene ricordare che esso è stato inteso come la ricerca di una terza via tra liberalismo classico e socialismo, ${ }^{85}$ alternativa a quella individuata dal

78 Marini (2011) 188.

79 Merryman (1973) 164.

80 Mezzetti (2009) $471 \mathrm{~s}$.

81 Mezzetti (2009) 472. Anche Rosenn (1971) 692.

82 Losano (2000) 177.

83 Kunz (1954) 213. Citazioni in Somma (2012).

84 VANDERLINDEN (1995) 258.

85 Carmagnani, Casetta (1989) 66 ss. 
fascismo europeo, ma per molti aspetti anche dal New Deal statunitense. ${ }^{\mathbf{8 6}}$ L'Europa fascista era infatti vista con sospetto e preoccupazione, e in tale contesto il nazionalismo diveniva «il vettore culturale che permise di adeguarsi, senza contrapporsi, al contesto internazionale negativo», consentendo di percorrere vie autonome rispetto alle drammatiche contrapposizioni dell'epoca. ${ }^{87}$

Questo per quanto concerne le deviazioni latinoamericane rispetto al modello occidentale di democrazia, che pure presentava all'epoca notevoli difetti o comunque ostacoli al suo radicamento. Quanto invece al mancato sviluppo del capitalismo, occorre considerare che esso era almeno fortemente indotto dalla politica economica statunitense. Proprio questo venne evidenziato alla conclusione del secondo conflitto mondiale dai teorici della dependencia nell'ambito della Commissione economica per l'America latina istituita presso le Nazioni Unite, i quali contestarono un assunto dell'economia neoclassica: che lo scambio economico tra centro e periferia avrebbe in un modo o nell'altro favorito la seconda. ${ }^{\mathbf{8}}$

Della situazione di dipendenza in cui si trovava e trova l'America latina si hanno numerosi riscontri fin dalla prima metà del Novecento. La politica economica statunitense era ampiamente retta dai principi della dottrina Monroe, che aveva però perso la sua carica anticolonialista per divenire lo scudo dietro il quale praticare politiche di egemonia sul continente americano. Era cioè una politica che mirava tra l'altro a privare l'America latina delle sue materie prime, impedendo che dai relativi proventi si sviluppasse una capacità produttiva autonoma nell'area: una politica in grado di incrementare la capacità di spesa dei latinoamericani, tuttavia solo per trasformarli in consumatori di prodotti finiti importati. ${ }^{89} \mathrm{Da}$ ciò la principale ragione dell'interesse manifestato dagli statunitensi per lo studio del diritto latinoamericano, ritenuto un fondamentale contributo allo sviluppo di una "politica di buon vicinato». ${ }^{90}$ Da ciò anche le critiche degli intellettuali latinoamericani, i quali fin dagli anni trenta del secolo scorso accusavano gli statunitensi di imporre forme di «economía destructiva», capaci solo di

86 Sulle somiglianze tra fascismo e New Deal, per tutti Schivelbusch (2008).

87 Carmagnani (2003) 303 ss.

88 Per tutti Dos Santos (1970) 231 ss.

89 Ad es. Pighini (1950) $10 \mathrm{s.}$

90 Cfr. Peters (1915-22) 208 ss. e Rasco (1949-50) 180 ss. 
produrre dipendenza economica e non anche di stimolare una «economía reproductiva y progresiva», fonte di una crescita duratura ed equilibrata. ${ }^{\mathbf{9 1}}$

In questo quadro si scordano volentieri alcuni primati latinoamericani che concernono entrambi i fronti in cui operano i processi di modernizzazione: democrazia e capitalismo. Quanto al fronte della democrazia, possiamo ricordare l'attenzione e il favore per «el magnifico principio de ugualdad de derechos de nacionales y extranjeros». ${ }^{92}$ Concerne invece il fronte del capitalismo la previsione da parte della Costituzione messicana del 1917 di un primo nucleo di diritti sociali, ${ }^{93}$ in anticipo sulla Costituzione di Weimar del $1919:{ }^{94}$ la prima Carta fondamentale europea a trattare questo aspetto. Se si adottassero i tradizionali schemi concernenti la circolazione dei modelli, si dovrebbe concludere che l'area europea continentale, cioè la porzione di occidente in cui si sono sviluppate discipline concernenti la democrazia economica e sociale, costituisce la periferia rispetto al centro latinoamericano. Ma, evidentemente, coloro i quali registrano il primato messicano non formulano certo retoriche in tal senso, che sarebbero altrimenti da condannare tanto quanto quelle volte a produrre il risultato opposto.

E' dunque l'etnocentrismo, strumento tipicamente utilizzato per imporre il «modello europeo di sviluppo economico a Paesi retti da economie preindustriali», ${ }^{95}$ l'ostacolo principale a una considerazione dell'esperienza giuridica latinoamericana che esuli da un suo inquadramento come esperienza periferica. Inquadramento cui, a ben vedere, allude anche la locuzione «America latina», che fa riferimento ai parlanti lingue europee, denunciando il proposito statunitense di appropriarsi del nome America, oltre all'intento di trascurare l'apporto degli indigeni e degli afroamericani.

Proprio a partire da una critica all'etnocentrismo della letteratura comparatistica sull'area latinoamericana, una recente classificazione di «grandi sistemi giuridici» si sofferma sulla necessità di dedicare una trattazione separata al «diritto dell'America meridionale». ${ }^{\mathbf{9 6}}$ Per non sacrificare i caratteri distintivi dei diversi ordinamenti nazionali dell'area, una simile trattazione

91 Uslar Pietri (1936).

92 Valladão (1954) 12.

93 Art. 123, comprendente fra l'altro l'obbligo dei datori di lavoro di soddisfare le esigenze abitative dei lavoratori, e inoltre di predisporre servizi sanitari ed educativi per la comunità.

94 Cfr. Ziskind (1984) 1 ss.

95 Losano (2000) 13 ss.

96 Losano (2000) 175 ss. 
dovrebbe tenere conto delle loro peculiarità, ma anche valorizzare vicende comuni, per poi consentire la ricostruzione di un'identità del diritto latinoamericano in termini alternativi a quelli ricorrenti nella retorica occidentale del selvaggio incapace di copiare.

La classificazione di cui parliamo valorizza la circostanza che l'identità del diritto latinoamericano è stata condizionata dalle caratteristiche del processo di colonizzazione, così come dalle vicende relative alla conclusione di quel processo. Quanto al primo aspetto, si sottolinea come la colonizzazione si sia espressa con modalità diverse da quelle successivamente assunte dall'imperialismo mercantile ottocentesco in area africana o asiatica. Per questo le popolazioni indigene sopravvissute allo sterminio sarebbero state assimilate e almeno formalmente poste sullo stesso piano delle popolazioni della madrepatria. Di qui lo sviluppo di particolari forme di pluralismo giuridico, ${ }^{97}$ seppure non nella forma della «stratificazione fra sistemi giuridici forti», ${ }^{98}$ comunque idonee a conferire tratti peculiari comuni all'esperienza latinoamericana.

Quanto ai caratteri dell'identità latinoamericana influenzati dalle modalità con cui è terminata la colonizzazione, si osserva che essa avvenne nello stesso torno di anni, quando le colonie sudamericane approfittarono dell'espansionismo francese in epoca napoleonica per liberarsi dalla dominazione iberica, e iniziare subito la costruzione di Stati nazionali indipendenti. ${ }^{99}$ Da qui le opportunità per lo sviluppo del capitalismo e della democrazia secondo dinamiche e contenuti tipici dell'area, incluso l'intreccio con la particolare forma di nazionalismo di cui abbiamo parlato.

Tutto ciò fornisce uno sfondo compiuto ai tentativi più classici di individuare l'identità del diritto latinoamericano, i primi dei quali risalgono almeno alla fine dell'Ottocento. ${ }^{\mathbf{1 0 0}}$ In questi tentativi si menzionano con più o meno enfasi elementi dell'identità riconducibili a una circolazione di modelli in particolare dal continente europeo. Non manca tuttavia la sottolineatura che essi si sono mescolati a elementi autoctoni o comunque specifici, come le consuetudini indigene, in forme tali da produrre un diritto dai tratti originali. ${ }^{101}$

97 WolKMer (2009) 297 ss.

98 Losano (2000) $176 \mathrm{~s}$. Tra chi propende per una lettura della conquista come non caratterizzata da pratiche assimilazioniste, v. ad es. Alvarado Velloso (1989) 2.

99 Losano (2000) 177.

100 Losano (2002) 389 ss.

101 Ad es. Gallo (1987) 296. 


\section{Segue: geopolitica e diritto indigeno}

La valutazione dell'identità giuridica latinoamericana, in quanto vicenda in cui si intrecciano dati geografici e schemi di ordine politico ed economico, presenta una indubbia valenza geopolitica, del resto immanente all'attività classificatoria tipica del comparatista. ${ }^{\mathbf{1 0 2}}$ Abbiamo riscontrato una simile valenza nei contributi che puntano a negare quell'identità, degradando il diritto latinoamericano a periferia di una tradizione o famiglia giuridica genericamente occidentale, o che eventualmente la affermano, ma solo per meglio consentire al centro di esercitare un controllo sulla periferia. Ci dedicheremo ora ai risvolti geopolitici dei contributi che puntano invece a celebrare l'identità latinoamericana, alcuni dei quali sono a ben vedere risalenti: come quelli da cui ha tratto fondamento il tentativo di edificare un diritto internazionale capace di arginare l'egemonia politica statunitense nell'area. ${ }^{103}$

Tralasceremo i contributi che, seppure sono dettati dal proposito di emancipare il diritto latinoamericano dallo statunitense, finiscono per non intaccare, e anzi per rafforzare, il rapporto di sudditanza rispetto al diritto europeo continentale. Questo accade in particolare con la sottolineatura della matrice romanistica dei diritti nazionali dell'area, attorno a cui sviluppare una sorta di resistenza latina. ${ }^{104}$ Quest'ultima possiede invero un marcata matrice neopandettista, in quanto tale inadatta a ribaltare l'idea di una periferia latinoamericana, debitrice nei confronti di un centro europeo continentale, la prima inesorabilmente destinata a importare i modelli definiti dal secondo.

Più opportuni appaiono i propositi di contrastare l'egemonia occidentale nel suo complesso, quindi anche l'egemonia culturale del Vecchio continente. ${ }^{105}$ In tal caso si muove dalla contestazione di quanto la tradizionale classificazione del diritto latinoamericano come periferia reputa essere una difettosa recezione dei modelli prodotti dal centro. ${ }^{106} \mathrm{E}$ si finisce per esaltare il costituzionalismo postcoloniale, con la sua tensione libertaria ed egualitaria, come espressivo delle «idee forza comuni a tutti i popoli di quelle

102 Cfr. Monateri (2013) 11 ss. e Somma (2014) 70 ss.

103 Alvarez (1910).

104 KLEINHEISTERKAMP (2008) $289 \mathrm{~s}$.

105 Mattei (2006) $820 \mathrm{~s}$.

106 Per tutti Esquirol (1997) 425 ss. e EsQuirol (2003) 41 ss. 
terre», ${ }^{\mathbf{1 0 7}}$ magari evidenziando i correttivi che si sono concepiti allorquando si è trattato di dare risposte alla questione sociale. In particolare questi aspetti, concernenti in ultima analisi il proposito di evidenziare «l'unità spirituale latinoamericana», sono stati valorizzati nella Dichiarazione di principi sull'insegnamento del diritto compilata sul finire degli anni cinquanta del secolo scorso dall'Unione delle università latinoamericane. ${ }^{\mathbf{1 0 8}}$

Occorre ora dire qualcosa sul diritto indigeno, finora menzionato come fondamento per la forma di pluralismo giuridico debole che contribuisce a definire l'identità giuridica latinoamericana. Questo diritto, la cui considerazione rileva indubbiamente dal punto di vista geopolitico, giacché attiene per definizione alla disciplina della convivenza di un gruppo umano che ha sviluppato una relazione particolarmente intima con il suo territorio, importa anche per un ulteriore aspetto: costituisce un imprescindibile punto di riferimento per riflettere sui termini della modernizzazione in area latinoamericana.

La riflessione può percorrere due strade. Per un verso può prendere spunto dalla circostanza che la modernizzazione si definisce essenzialmente come superamento dell'elemento tradizionale o comunque premoderno: da un simile punto di vista la componente indigena dell'identità latinoamericana restituisce la distanza di quell'identità dalla modernizzazione tout court. Per un altro verso si possono invece valorizzare i tentativi di discutere di modernizzazioni al plurale, si può cioè considerare l'intreccio tra diritto indigeno e sviluppo della democrazia e del capitalismo come il tratto distintivo della modernizzazione latinoamericana rispetto alle altre forme di modernizzazione, quella occidentale in testa. E' quest'ultimo il punto di vista che utilizzeremo, e che completeremo fra breve approfondendo i termini dello sviluppo della democrazia e del capitalismo in area latinoamericana.

Per trattare questi aspetti, ci riferiremo a una recente dottrina comparatistica che ha legato le sue fortune all'utilizzo della tradizione giuridica come punto di riferimento per una classificazione alternativa a quelle incentrate sui caratteri del diritto nazionale. Nel merito si menziona la «tradizione ctonia», locuzione in buona sostanza utilizzata per intendere la tradizione giuridica primordiale, quella che «semplicemente è emersa perché l'esperienza si è

107 Alvarado Velloso (1989) 11.

108 First Conference of Latin-American Law Schools, in: Inter-American Law Review 1 (1959) 241. 
accumulata, e la memoria e la sua trasmissione orale hanno fatto il loro lavoro». Ebbene, la tradizione ctonia è l'unica a essere menzionata come tratto specifico dell'area latinoamericana, assieme ad alcuni suoi caratteri che costituiscono ancora oggi un punto di riferimento per identificare il nucleo essenziale del diritto indigeno di quell'area: il culto per l'armonia tra i componenti il gruppo umano, così come tra quest'ultimo e l'ambiente naturale. ${ }^{109}$

La stessa dottrina discute poi della tradizione di civil law, per affermare che «la sua influenza storica nel mondo appare inconfondibile», ma anche che essa non è dovuta unicamente alla "semplice ammirazione», e dunque al prestigio. La tradizione di civil law viene infatti dipinta come violenta e imperialista, «associata all'idea di dominazione», tanto da indurre a chiedersi se «gli occidentali sono essenzialmente dei fondamentalisti, a tal punto da ritenere che le loro soluzioni siano talmente vere da dover essere seguite ovunque». ${ }^{110}$

Queste precisazioni sembrano preludere all'impossibilità di combinare le due tradizioni in forme capaci di delineare una specifica modernità latinoamericana. Invero la tradizione di civil law è «relazionale», incentrata come è sul rapporto tra individui portatori di «diritti soggettivi». Al contrario, nella tradizione ctonia «'individuo è immerso nel passato e nella comunità, motivo per cui non è concepibile il «potere di ottenere l'oggetto della volontà individuale», non sono concepibili «diritti» e neppure «interessi individuali» tutelati dall'ordinamento: la tradizione «fa quadrato contro di essi». ${ }^{\mathbf{1 1 1}} \mathrm{Da}$ ciò la presunta carica antimodernista del diritto indigeno e dunque la sua radicale alterità rispetto al progetto della modernizzazione, soprattutto con riferimento agli aspetti concernenti lo sviluppo in senso capitalista.

A ben vedere, le cose non possono essere rappresentate nei termini di una così radicale contrapposizione. La modernizzazione capitalista, come vedremo fra breve, ha storicamente prodotto costruzioni tutt'altro che incentrate sull'individuo e sulle sue strategie di emancipazione. Ha cioè favorito la funzionalizzazione delle libertà economiche del singolo nelle forme di volta in volta ritenute necessarie ad assicurare l'equilibrio e lo sviluppo dell'ordine proprietario, mettendo in luce come il carattere della relazionalità non fosse $\mathrm{e}$

109 GLenN (2000) 56 ss.

110 GLenN (2000) 153.

111 GLenN (2000) 67 e $130 \mathrm{~s}$.

32 Alessandro Somma 
non sia capace di produrre un rapporto tra individuo e ordine non troppo sbilanciato verso quest'ultimo.

Più recentemente si sono poi registrate novità importanti per chi coltiva «l'oscuro desiderio» di individuare «diritti collettivi», ovvero costruzioni che uniscono «individualità» e «socialità», ${ }^{112}$ che combinano tensione comunitarista e cultura dei diritti, inclusi quelli di natura economica. In tal senso dispone la Dichiarazione dei diritti dei popoli indigeni approvata dall'Assemblea generale delle Nazioni Unite nel 2007 che, oltre a tutelare una serie di diritti dei «singoli individui indigeni» anche nei confronti della loro comunità, riconosce fra l'altro il diritto collettivo «alle terre, territori e risorse che hanno tradizionalmente posseduto, o occupato o altrimenti utilizzato o acquisito» $^{113}$

La frizione tra diritto indigeno e modernità non è dunque insanabile: è al contrario foriera di modalità alternative di intendere la modernità. Il tutto secondo schemi che sono anzi al centro dell'attuale dibattito tra i cultori del diritto impegnati a produrre per l'ordine economico regole concernenti la sua sostenibilità anche dal punto di vista democratico: in particolare regole concernenti la dissociazione tra titolarità e amministrazione della proprietà cui prelude la teoria dei beni comuni, a ben vedere riconducibile a risalenti e ora screditate riflessioni occidentali dedicate al tema della democrazia economica. ${ }^{\mathbf{1 1 4}}$

Giungiamo così a toccare il tema della modernizzazione come vicenda che occorre analizzare con le lenti del pluralismo, utili tra l'altro a individuare più centri da cui essa prende corpo, magari proprio laddove l'impostazione tradizionale pretende di ravvisare una semplice periferia.

\section{Dalla modernizzazione alle modernità: alternative, parallele e multiple}

Come abbiamo detto, spesso e volentieri i teorici della modernizzazione hanno intrecciato le loro riflessioni con schemi di matrice evoluzionista. Non lo hanno fatto sempre: in un certo senso i primi contributi sul tema presupponevano almeno implicitamente una visione pluralista del fenome-

112 López Calera (2000) 167.

113 Art. 26. Cfr. Xanbthaki (2007) 29 ss.

114 Cfr. Sомma (2011a) 461 ss. 
no, studiato soprattutto con riferimento all'area europea, di cui si finivano per mettere in luce alcune differenze anche rilevanti tra diverse modalità con cui si delineava e realizzava il programma della modernizzazione. ${ }^{\mathbf{1 1 5}}$

Evoluzionista fu invece l'ambiente culturale in cui vide la luce e si sviluppò la comparazione giuridica. Forse una simile attitudine non fu sempre la ricaduta di un consapevole programma culturale, almeno non tanto quanto lo fu a partire dalla conclusione del secondo conflitto mondiale nell'ambito del movimento di Law and development. Con esso la modernizzazione, così come si era affermata in area occidentale, venne di fatto eretta a parametro della modernizzazione tout court, da utilizzare come punto di riferimento per lo sviluppo delle altre aree del pianeta.

Un simile approccio ha resistito a lungo, magari celato dietro professioni di diffusionismo, che tuttavia non hanno inciso in modo significativo su un atteggiamento di fondo di matrice etnocentrica. Eppure molte vicende documentavano e documentano l'impraticabilità di un'attitudine evoluzionista allo studio della modernizzazione, così come il carattere ideologico di quell'attitudine, con riferimento a entrambi gli aspetti del fenomeno: lo sviluppo del capitalismo, esattamente come della democrazia.

Quanto al secondo aspetto, lo sviluppo della democrazia, non poche vicende hanno definitivamente chiarito come la modernizzazione non lo implicasse necessariamente. La democrazia ben poteva essere ritenuta un orizzonte tenuto in conto dalla società borghese, che aveva a tal fine edificato la società del diritto privato, fondata sull'identificazione della libertà con la condizione proprietaria, universalmente accessibile attraverso il lavoro. ${ }^{\mathbf{1 1 6}}$ Le cose erano però destinate a cambiare con la transizione verso la società industriale, fondata sulla weberiana «schematizzazione coercitiva dell'esistenza», ${ }^{117}$ o sulla durkheimiana differenziazione funzionale della società. ${ }^{118}$ Questa transizione cambiò il lavoro, che da strumento di emancipazione individuale mutò in attività preposta al sostegno dell'equilibrio e dello sviluppo dell'ordine proprietario, in quanto tale capace di produrre più inclusione funzionalizzante che mobilità sociale.

115 Citazioni in Eisenstadt (1990) 17 ss.

116 Sul punto ad es. Costa (2005) 33 ss.

117 WeBER (2000) 85.

118 Durkheim (1926) part. 189 ss.

$34 \quad$ Alessandro Somma 
E la «schematizzazione coercitiva dell'esistenza» ben poteva richiedere il sacrificio delle libertà politiche come presupposto per la riforma delle libertà economiche in senso capitalista. Questo avvenne per le esperienze in cui la modernizzazione fu avviata o realizzata nell'ambito di sistemi autoritari, come ad esempio nell'esperienza prussiana, per la quale fu nel merito formulata la cosiddetta teoria del percorso eccezionale o Sonderweg. ${ }^{119}$ Lo stesso dicasi poi, e soprattutto, per l'esperienza fascista, che si sviluppò nel momento in cui i conflitti prodotti dal sostegno ai processi di modernizzazione imposero di ristabilire la pace sociale con forme di inclusione violenta dell'individuo nell'ordine proprietario. ${ }^{\mathbf{1 2 0}}$

Il nesso tra modernizzazione e democrazia è dunque instabile, a tal punto che la mancanza di trasformazioni in quel senso non indica semplicemente la presenza di una «soglia critica dello sviluppo politico». ${ }^{121}$ Quella mancanza conferma più propriamente che il liberalismo costituisce una teoria relativa al modo di combinare profitto individuale e collettivo, e non certo una teoria sulla libertà illimitata: una pratica governamentale che si regge sulla produzione della libertà, ma anche e necessariamente sul suo consumo. ${ }^{\mathbf{1 2 2}}$

Peraltro anche il capitalismo non costituisce una costante nei processi di modernizzazione, o almeno non il capitalismo inteso come una teoria e una pratica riconducibili ad unità. Questo aspetto è stato a ben vedere tematizzato solo dopo il fallimento del socialismo, ovvero nel momento in cui cessava l'aspro confronto tra blocchi identificati dalla condivisione o meno dell'opzione capitalista. Con la fine di quel confronto fu evidente l'esistenza di uno scontro tra modelli di capitalismo, in particolare tra un capitalismo renano e un capitalismo anglosassone: il primo qualificato da una spesa sociale elevata e dalla centralità del sistema bancario, il secondo da una spesa sociale ridotta e dalla centralità dei mercati finanziari. ${ }^{123}$ Similmente altri hanno discusso di un capitalismo del welfare contrapposto a un capitalismo della borsa, ${ }^{\mathbf{1 2 4}}$ così come di economie di mercato coordinate e di economie di mercato liberali. $^{125}$

119 Teoria controversa, su cui WeHLER (1998) $78 \mathrm{~s}$.

120 Citazioni in Somma (2005b) 81 ss.

121 Martinelli (2002) 53 ss.

122 Così Foucault (2007) 65 e $264 \mathrm{~s}$.

123 Cfr. Albert (1991).

124 Dore (2000).

125 Hall, Soskice (2001). 
E' senz'altro vero che queste contrapposizioni si fondano in parte su dati reperiti prima della fine del socialismo, e che le recenti vicende hanno mutato di molto i termini dello scontro tra modelli di capitalismo, mostrando l'opzione anglosassone come opzione vincente a livello planetario per effetto dei processi di globalizzazione: ${ }^{126}$ questo si ricava anche dai successi della menzionata New comparative economics, utilizzata ad esempio come punto di riferimento per l'attuale ristrutturazione del debito sovrano delle economie sudeuropee. ${ }^{\mathbf{1 2 7}}$ Altrettanto vero è che, se si considerano i «regimi di accumulazione», ovvero i modelli di produzione e consumo stabili nel tempo, le forme di capitalismo ricorrenti in area anglosassone e quelle tipiche dell'Europa continentale mostrano differenze molto meno marcate di quelle che emergono considerando i soli «modi di regolazione», intesi come l'architettura istituzionale necessaria a stabilizzare quei modelli di produzione e consumo. ${ }^{128}$

Peraltro la considerazione per il contesto in cui operano i regimi di accumulazione, che pure viene invocata da chi opera la distinzione appena tracciata, ${ }^{129}$ conduce a ritenere la diversità tra capitalismi come una vicenda ineliminabile, tanto quanto il carattere impositivo della loro circolazione, piuttosto che il suo essere determinata dal prestigio dell'opzione vincente. Il che si ricava valorizzando, oltre alle vicende successive al crollo del socialismo, anche quelle precedenti, quindi l'epoca d'oro dell'approccio evoluzionista alla modernizzazione.

Esemplificativo, in quanto coinvolse l'occidente capitalista, il dibattito alla conclusione della seconda guerra mondiale sulla costituzione economica della rinata democrazia tedesca, che vide il confronto di due modelli contrapposti. Vi era da un lato il modello della «democrazia economica», fondato su una forma di pianificazione e sull'intervento statale in chiave redistributiva anche entro i confini del mercato, ritenuto indispensabile a produrre emancipazione individuale. E vi era dall'altro il modello della «democrazia neoliberale», per cui l'intervento statale doveva essere rivolto alla sola definizione del quadro normativo necessario e sufficiente ad assicurare il funzio-

126 Già Crouch, Streeck (1997) 1 ss.

127 Notizie in Somma (2014).

128 La distinzione tra regimi di accumulazione e modi di regolazione è sviluppata nell'ambito della Régulation theory: ad es. Boyer, SAILlard (2002) $36 \mathrm{f}$.

129 Cfr. Boyer (2004) 7. 
namento del meccanismo concorrenziale, ritenuto il migliore strumento di redistribuzione della ricchezza. ${ }^{\mathbf{1 3 0}}$ Il tutto a dimostrazione ultima che lo scontro tra capitalismi è immanente allo stesso sviluppo del capitalismo come tratto della modernizzazione: che dunque non può concepirsi in termini monolitici o unitari, come sembrano invece sottintendere coloro i quali discorrono di modernizzazione e tradizione giuridica occidentale.

Se così stanno le cose, non sembra possa prescindersi dal considerare la modernità in termini pluralistici, così come dallo stemperare la contrapposizione tra società moderna e società tradizionale, quest'ultima ritenuta un ostacolo insormontabile all'avvento della prima. In quest'ultimo senso si riconosce ora che aspetti della modernità si possono esprimere anche in società dai più reputate tradizionali, ad esempio per il persistere di un ruolo del credo religioso incompatibile con quanto classicamente si associa all'idea di secolarizzazione. Proprio in questo senso si argomenta nell'ambito della New traditional economy, approccio volto a valorizzare le esperienze in cui si combinano «il vecchio con il nuovo, l'individuale con il collettivo, l'etico con il pratico». ${ }^{131}$

Possiamo considerare questi rilievi come introduttivi dei concetti di «modernità alternative», "parallele» o ancora «multiple». Il primo è stato elaborato per sottolineare che la modernizzazione non può alimentare una «teoria della convergenza» delle culture, giacché «diversi punti di partenza per la transizione verso la modernità» non possono che condurre a «diversi risultati». ${ }^{132}$ Chi discorre di modernità parallele mette in luce l'essenza di una simile diversità, in qualche modo riconducibile al modo di manifestarsi del pluralismo in quanto relativo alla «coesistenza nello spazio e nel tempo di molteplici flussi economici, culturali e religiosi». ${ }^{133}$ Il che porta a discorrere della modernità come di un fenomeno multiplo, risultante cioè dal succedersi di «continue costituzioni e ricostituzioni di una molteplicità di programmi culturali», il cui esito costituisce una variabile dipendente dalle caratteristiche dei contesti in cui si verificano simili costituzioni e ricostituzioni: caratteristiche concernenti il modo di essere della convivenza sociale, ma anche della complessiva visione del mondo. ${ }^{\mathbf{1 3 4}}$

130 Citazioni in Somma (2011b) 1 ss.

131 Rosser, Rosser (2004) $86 \mathrm{~s}$.

132 Per tutti Gaonkar (1999) $1 \mathrm{ss.}$

133 Ad es. Larkin (1997) 407 ss.

134 Eisenstadt (2002) 1 ss. 
In tale prospettiva la diffusione della modernità, per quanto si riveli «inevitabile», ${ }^{135}$ cessa di coincidere con l'occidentalizzazione dei contesti in cui si manifesta. Contesti nei quali non si verificano meri recepimenti, bensì, quantomeno, contaminazioni tra influenze esterne comunque esercitate e dati identitari interni in perenne trasformazione.

\section{A mo' di conclusione: modernità multiple, individuo e ordine}

Abbiamo detto delle critiche oramai definitive all'idea della modernità come vicenda prodotta da un centro e importata da una periferia: idea combattuta dai teorici della dependencia, secondo schemi ripresi dai teorici del «sistema mondo», ${ }^{136}$ e dalla critica postcoloniale. ${ }^{137}$ Peraltro, se anche si accede all'idea per cui la modernità deve essere letta al plurale, occorre chiedersi se vi sia un nucleo della modernità, come vicenda capace, pur prescindendo dallo schema per cui essa si diffonde dal centro verso la periferia, di accomunare le sue molteplici manifestazioni.

Almeno implicitamente, una riflessione su questo aspetto affonda le sue radici nel tempo. ${ }^{\mathbf{1 3 8}}$ Da molto la riflessione sociologica ha infatti concepito la modernità come un processo che era in buona sostanza perennemente inconcluso, quindi come una vicenda che poneva di fronte a oggetti in continuo divenire. Un vicenda in cui si combinavano in modo variabile e selettivo, assieme allo sviluppo del capitalismo e alla democrazia, tendenze come la coscienza dei propri bisogni e la rivendicazione degli strumenti per soddisfarli, la secolarizzazione e dunque l'espulsione del trascendente dal modo di concepire i termini della convivenza sociale. Il tutto accompagnato da trasformazioni come la differenziazione funzionale della società, l'incremento demografico e l'urbanizzazione, la comunicazione, il consumo e la cultura di massa, e molte altre.

Ebbene, le analisi fin qui viste, anche quelle viziate da evoluzionismo magari travestito da diffusionismo, sono sufficienti a restituire l'immagine della modernità come vicenda il cui nucleo non annovera stabilmente la democrazia come tratto caratteristico. E a farci apparire il capitalismo come

135 GaONKar (1999) 1.

136 Sulla scia di Immanuel Wallerstein, di cui v. da ultimo WaLlerstein (2004).

137 Su cui la letteratura è sterminata. V. ad es. Mellino (2005).

138 JeDLOWSKI (2011) 96.

$38 \quad$ Alessandro Somma 
costante della modernità solo ove lo si riduca alla weberiana razionalizzazione dell'esistenza, alla base dei processi di differenziazione funzionale della società: ovvero al «macchinismo», ${ }^{139}$ o eventualmente a vicende come l'urbanizzazione o la diffusione dei moderni sistemi di comunicazione. ${ }^{140}$ Giova a questo punto concentrare l'attenzione sulle modalità attraverso cui si esprime la razionalizzazione dell'esistenza nella modernità latinoamericana, questa volta non per esaltarne l'identità, bensì per mettere in luce le equivalenze funzionali rispetto alla modernità occidentale, che identificheremo valorizzando fra l'altro la dimensione discorsiva nella definizione del rapporto tra diritto e società.

Come abbiamo detto, «caudillismo» è l'espressone utilizzata per esemplificare i tratti del sistema politico affermatosi in area latinoamericana fin dall'epoca in cui essa ha ottenuto l'indipendenza dai colonizzatori europei. E' un'espressione che un osservatore statunitense di metà Novecento riteneva indicasse i tratti tipici, quasi antropologici, dei latinoamericani, da sempre «più interessati ai loro uomini pubblici che alle loro politiche». ${ }^{141} \mathrm{Da}$ ciò la venatura autoritaria del sociale come portato del nazionalismo interclassista tipico di questa porzione del pianeta, ${ }^{\mathbf{1 4 2}}$ alla base di un sistema di potere che, confrontato con quello occidentale, ha condotto a ritenere il presidenzialismo latinoamericano come degenerazione del modello imitato, soprattutto nella fase successiva alla crisi economica del $1929 .{ }^{\mathbf{1 4 3}}$

Eppure ciò che viene identificata come identità negativa della periferia latinoamericana riassume caratteri che non sono certo assenti in capo al centro $^{144}$ e che, ove assenti, possono pur sempre riemergere, a meno che non si reputi che la democrazia in area europea sia un dato acquisito e irreversibile. Ma questa considerazione, oltretutto smentita dal dato storico, avrebbe un sapore marcatamente evoluzionista, esattamente come il ritenere irreversibile il riconoscimento dei diritti sociali, dopo la conquista di quelli civili e politici. ${ }^{145}$

JedLowski (2011) 106.

140 Eisenstadt (1990) 54.

141 Macdonald (1949) 7 ss.

142 Al proposito MarinI (2011) 177.

143 Mezzetti (2009) 474.

144 Esquirol (2011) 250.

145 Cfr. Marshall, Bottomore (1992). 
E' sicuramente vero che in America latina il caudillismo ha prodotto una commistione di nazionalismo e socialismo, e che questa è stata intesa come fondativa dei processi di modernizzazione nell'area, quindi posta al servizio delle dinamiche di razionalizzazione destinate a condizionare lo sviluppo capitalista. In questo senso devono essere intese vicende come la costruzione di un modello educativo «tecnicista» o di relazioni industriali «militariste», ${ }^{146}$ che pure concernevano ambiti ricompresi nel sistema dei diritti sociali contemplato dal costituzionalismo latinoamericano, e che tuttavia erano destinati a produrre lo scioglimento dell'individuo nell'ordine in luogo della sua emancipazione. Tutto questo è vero, ma non definisce tratti identitari tipici dell'area latinoamericana o comunque estranei all'area europea.

In quest'ultima, infatti, finalità assimilabili a quelle appena descritte sono state perseguite attraverso lo sviluppo dello Stato sociale, come ben si ricava dalle motivazioni addotte per la sua edificazione, ovvero che «il sostegno attivo al benessere dei lavoratori» costituiva, al pari della «repressione dei tumulti», un modo per ottenere «la riparazione dei danni sociali» prodotti dalla modernizzazione. ${ }^{147} \mathrm{E}$ difatti la previsione di diritti sociali come fondamento per l'erogazione di prestazioni sociali fu il tratto identificativo del costituzionalismo del dopoguerra, che voleva in tal modo prevenire il ritorno del totalitarismo fascista, tutto incentrato sul ricorso alle prestazioni sociali come strumento di pacificazione e di inclusione forzata dell'individuo nell'ordine.

Altrimenti detto, i diritti sociali sono sorti come strumento attraverso cui rendere le prestazioni sociali un veicolo di emancipazione dell'individuo, piuttosto che di funzionalizzazione dei suoi comportamenti per finalità concernenti l'equilibrio e lo sviluppo dell'ordine proprietario. Il tutto, però, sulla base di intendimenti che sono ora fortemente ridimensionati, tanto da far ritenere, finalmente in chiave non evoluzionistica, che il sistema dei diritti sociali non sia più un tratto identificativo dell'esperienza giuridica europea. ${ }^{148}$

Se così stanno le cose, la descrizione dell'esperienza latinoamericana come incentrata su forme di autoritarismo al servizio di una «modernizzazione

Cfr. Mansilla (2007) 59 ss.

147 Kaiserliche Botschaft di Guglielmo I di Prussia del 17 novembre 1881, in: Stenographische Berichte (1882) 1 ss.

148 Diffusamente Somma (2009) 131 ss. 
imitativa» ${ }^{\mathbf{1 4 9}}$ non identifica certo tratti estranei all'esperienza utilizzata a tal fine come modello. Anche in quest'ultima sono da tempo diffusi schemi che puntano a sostenere la modernizzazione comprimendo i relativi conflitti attraverso il ricorso a forme di scioglimento dell'individuo nell'ordine: forme certo meno cruente di quelle ricorrenti in area latinoamericana, ma non per questo meno efficaci dal punto di vista dello scioglimento dell'individuo nell'ordine. E' ad esempio questa l'essenza dell'economia sociale di mercato, sviluppo della democrazia neoliberale, ${ }^{\mathbf{1 5 0}}$ menzionata a proposito dello scontro tra capitalismi che ha caratterizzato il mondo occidentale.

Alla luce di tutto ciò occorre valutare l'intreccio tra cultura indigena da un lato, e sviluppo del capitalismo e della democrazia dall'altro, come possibile tratto identificativo della modernizzazione latinoamericana. La cultura indigena non può cioè essere degradata a mera «etnicità politicizzata», capace di produrre modernità solo ove sia adattabile «ai modelli organizzativi di Stato, Nazione, popolo e cittadinanza che si sono diffusi in tutto il mondo negli ultimi due secoli». ${ }^{\mathbf{1 5 1}}$ Certo, è una cultura capace di preludere allo scioglimento dell'individuo nell'ordine, non tuttavia se combinata con le forme di conflitto identificative della modernizzazione democratica, attraverso cui far emergere la valenza emancipatoria delle tensioni cooperative, anche e soprattutto nelle relazioni con l'ambiente naturale. Per poi realizzare forme di convivenza sociale di cui auspicare la circolazione dal Nuovo al Vecchio mondo.

\section{Bibliografia}

Abert, K. H. (1978), Rechtsvergleichung, Bern

Agostini, E. (1988), Droit comparé, Paris

Albert, M. (1991), Capitalisme contre capitalisme, Paris

Alvarado Velloso, A. (1989), Voce Diritto dei Paesi latino-americani, in: Enciclopedia giuridica Treccani, vol. 6, Roma

Alvarez, A. (1910), Le droit international américain. Son fondement, sa nature d'après l'histoire diplomatique des États du Nouveau monde et leur vie politique et économique, Paris

149 Mansilla (1997).

150 Per tutti Haselbach (1991) part. 113 ss.

151 Brubaker (2011) 93. 
Arminjon, P., B. Nolde, M. Wolff (1950), Traité de droit comparé, vol. 1, Paris Barton, J. H. et al. (1983), Law in Radically Different Cultures, St. Paul Minn.

Benedix, R. (1964), Nation-Building and Citizenship, Berkeley

Berman, H. J. (1983), Law and Revolution, Cambridge, London

Boyer, R. (2004), How and Why Capitalism Differs, Max-Planck-Institut für Gesellschaftsforschung Discussion paper 05/2004, www.mpifg.de/pu/mpifg_dp/ dp05-4.pdf

Boyer, R., Y. Saillard (2002), A Summary of Regulation Theory, in: Boyer, R., Y. SAIL-LARD (a cura di), Regulation Theory: The State of the Art, London, New York

Brubaker, R. (2011), Nazionalismo, etnicità e modernità, in: Corradi, C., D. PacelLI (a cura di), Dalla modernità alle modernità multiple. Percorsi di studio su società e culture, Soveria Mannelli

Burg, E. M. (1977), Law and Development, in: American Journal of Comparative Law 25

Carmagnani, M. (2003), L'altro Occidente, Torino

Carmagnani, M., G. Casetta (1989), America latina: la grande trasformazione 1945-1985, Torino

Cavalli, A. (1998), Voce Tradizione, in: Enciclopedia Treccani delle Scienze sociali, vol. 8, Roma

Costa, P. (2005), Cittadinanza, Roma, Bari

Crouch, C., W. Streeck (1997), The Future of Capitalist Diversity, in Crouch, C., W. Streeck (a cura di), Political Economy of Modern Capitalism. Mapping Convergence and Diversity, London

David, R. (1950), Traité élémentaire de droit civil comparé, Paris

David, R., C. Jauffret-Spinosi (2002), Les grands systèmes de droit contemporains, 11. ed., Paris

De Cruz, P. (1999), Comparative Law in a Changing World, 2. ed., London, Sydney DJankov, S. et al. (2003), The New Comparative Economics, in: Journal of Comparative Economics 31

Dölemeyer, B. (2010), Rechtsräume, Rechtskreise, in: Europäische Geschichte Online, www.ieg-ego.eu/doelemeyerb-2010-de

Dore, R. P. (2000), Stock Market Capitalism - Welfare Capitalism, Oxford

Dos Santos, Th. (1970), The Structure of Dependence, in: American Economic Review 60, 231 ss.

Esmein, A. (1900), Le droit comparé et l'enseignement du droit, in: Nouvelle Revue historique de droit français et étranger 24

Durkheim, E. (1926), De la division du travail social (1893), 5. ed., Paris

Eisenstadt, S. N. (1990), Civiltà comparate. Le radici storiche della modernizzazione (1981-83), Napoli

Eisenstadt, S. N. (2002), Multiple Modernities, in: Eisenstadt, S. N. (a cura di), Multiple Modernities, New Brunswick N.J.

Esquirol, J. L. (1997), The Fictions of Latin American Law, in: Utah Law Review 50

42 Alessandro Somma 
Esquirol, J. L. (2003), Continuing Fictions of Latin American Law, in: Florida Law Review 55

Esquirol, J. L. (2011), Il fallimento del diritto latinoamericano come tradizione, in: Rivista critica del diritto privato 29

Feldman, E. A. (1997), Patients' Rights, Citizens' Movements and Japanese Legal Culture, in: Nelken, D. (a cura di), Comparing Legal Cultures, Aldershot

Foucault, M. (1999), L'archeologia del sapere (1969), Milano

Foucault, M. (2007), Nascita della biopolitica (1978-79), Milano

Friedman, L. M. (1978), Il sistema giuridico nella prospettiva delle scienze sociali (1975), Bologna

Frobenius, L. (1897-1898), Der westafrikanische Kulturkreis, in: Petermanns Geographische Mitteilungen 42-43

Fromont, M. (1998), Grands systèmes de droit étrangers, 3. ed., Paris

Gallo, P. (1987), Voce America latina, in: Digesto delle Discipline privatistiche Sezione civile, vol. 1, Torino

Gambaro, A., R. Sacco (1996), Sistemi giuridici comparati, Torino

Gaonkar, D. P. (1999), On Alternative Modernities, in: Public Culture 11, 1

Gardner, J. A. (1980), Legal Imperialism, Madison Wisc.

Giaro, T. (2003), Oriente e Occidente nella storia del diritto privato europeo, in:

Alpa, G., R. Danovi (a cura di), Diritto privato europeo, Milano

Glendon, M. A., M. W. Gordon, P. G. Carozza (1999), Comparative Legal Traditions, St. Paul Minn.

Glenn, H. P. (2000), Legal Traditions of the World, Oxford

Gordley, J. (2003), Common law und civil law: Eine überholte Unterscheidung, in: Zeitschrift für Europäisches Privatrecht 11

Grande, E. (2000), Imitazione e diritto, Torino

Grisoli, A. (1962), Corso di diritto privato comparato, Milano

Hall, P. A., D. Soskice (2001), Varieties of Capitalism, Oxford

Haselbach, D. (1991), Autoritärer Liberalismus und soziale Marktwirtschaft, BadenBaden

Hobsbawm, E. (2002), Come si inventa una tradizione, in: Hobsbawm, E., T. Ranger (a cura di), L'invenzione della tradizione (1983), Torino

Jedlowski, P. (2011), Modernità multiple: quale molteplicità?, in: Corradi, C., D. PACELli (a cura di), Dalla modernità alle modernità multiple. Percorsi di studio su società e culture, Soveria Mannelli

Kleinheisterkamp, J. (2008), Development of Comparative Law in Latin America, in: Reimann, M., R. Zimmermann (a cura di), The Oxford Handbook of Comparative Law, Oxford, 263-301

Kӧтz, H. (1998), Abschied von der Rechtskreislehre?, in: Zeitschrift für Europäisches Privatrecht 6

Kunz. J. L. (1954), Contemporary Latin-American Philosophy of Law, in: American Journal of Comparative Law 3

Larkin, B. (1997), Indian Films and Nigerian Lovers. Media and the Creation of Parallel Modernities, in: Africa 67 
Legeais, R. (2004), Grands systèmes de droit contemporains, Paris

Legrand, P. (1999), Le droit comparé, Paris

Legrand, P. (2001), What «Legal Transplants»?, in: Nelken, D., J. Feest (a cura di), Adapting Legal Cultures, Oxford e Portland

López Calera, N. (2000), Hay derechos colectivos?, Barcelona

Lopez Medina, D. E. (2004), Teoría impura del derecho, Bogotá

López Medina, D.E. (2011), La teoria impura del diritto: le trasformazioni della cultura giuridica latinoamericana, in: Rivista critica del diritto privato 29

Losano, M. (2000), I grandi sistemi giuridici. Introduzione ai diritti europei ed extraeuropei, Roma, Bari

Losano, M. (2002), Clóvis Bevilaqua entre comparação e filosofia do direito, in: VII Congresso Brasileiro de Filosofia, João Pessoa, 389 ss.

Macdonald, A. F. (1949), Latin American Politics and Government, New York

Mansilla, H.C. F. (1997), Tradición autoritaria y modernizacion imitativa, La Paz

Mansilla, H. C. F. (2007), Socialismo y nacionalismo come agentes de modernización acelerada, in: Cuadernos del Cendes 24

Marini, G. (2011), La costruzione delle tradizioni giuridiche e il diritto latinoamericano, in: Rivista critica del diritto privato 29

Marshall, T. H., T. Bottomore (1992), Citizenship and Social Class, London

Martinelli, A. (2002), La modernizzazione, 6. ed., Roma, Bari

Mattei, U. (2008), Comparative Law and Critical Legal Studies, in: Reimann, M., R. Zimmermann (a cura di), The Oxford Handbook of Comparative Law, Oxford, 815-836

Mattei, U. (1997), Three Patterns of Law: Taxonomy and Change in the World's Legal Systems, in: American Journal of Comparative Law 45

Mattei, U., P. G. Monateri (1997), Introduzione breve al diritto comparato, Padova

Mellino, M. (2005), La critica postcoloniale, Roma

Merryman, J.H. (1973), La tradizione di civil law (1969), Milano

Merryman, J. H. (1977), Comparative Law and Social Change, in: American Journal of Comparative Law 25

Mezzetti, L. (2009), L'America latina, in: Carrozza, P., A. Di Giovine, G. F. Ferrari (a cura di), Diritto costituzionale comparato, Roma, Bari

Mirow, M.C. (2009), Voce South and Central America: Overview, in: Oxford International Encyclopedia of Legal History, vol. 5, Oxford

Monateri, P. G. (2013), Geopolitica del diritto, Roma, Bari

Nelken, D. (2007), Defining and Using the Concept of Legal Culture, in: Örücü, E., D. Nelken (a cura di), Comparative Law, Oxford, Portland

Orestano, R. (1987), Introduzione allo studio del diritto romano, Bologna

Pargendler, M. (2012), The Rise and Decline of Legal Families, in: American Journal of Comparative Law 60

Peters, A. G. (1915-22), Importance of the Study of Latin-America Law, in: American Law School Review 4, 208 ss.

Pighini, G. (1950), Venezuela Paese dell'avvenire, Firenze

$44 \quad$ Alessandro Somma 
Rasco, R. A. (1949-50), The Need for a Latin-American Program in the Law School Curriculum, in: Journal of Legal Education 2

Rheinstein, M. (1987), Einführung in die Rechtsvergleichung, 2. ed., München

Rosenn, K. S. (1971), Teaching Latin American Law, in: American Journal of Comparative Law 19

Rosser, J. B., M.V. Rosser (2004), Comparative Economics in a Transforming World Economy, 2. ed., Cambridge, London

Rosti, M. (2007), Sull'esistenza di un sistema giuridico ibero-americano, in: Cardozo Electronic Law Bulletin 13

Rouland, N. (1998), Introduction historique au droit, Paris

SACCo, R. (1992), Introduzione al diritto comparato, Torino

Sarfatti, M. (1933), Introduzione allo studio del diritto comparato, Torino

Schipani, V. S. (a cura di) (1981), Diritto romano, codificazione e sistema giuridico latino-americano, Milano

Schivelbusch, W. (2008), 3 New Deal, Milano

Schlesinger, R. B. et al. (1998), Comparative Law, 6. ed., New York

Société D’Études législatives (1904), Le Code civil 1804-1904 - Livre du Centenaire, Paris

Somma, A. (2001), L'uso giurisprudenziale della comparazione nel diritto interno e comunitario, Milano

Somma, A. (2005a), Da Roma a Washington, in: Monateri, P. G., T. Giaro, A. Somma, Le radici comuni del diritto europeo, Roma

Somma, A. (2005b), I giuristi e l'Asse culturale Roma-Berlino. Economia e politica nel diritto fascista e nazionalsocialista, Frankfurt M.

Somma, A. (2005c), Tecniche e valori nella ricerca comparatistica, Torino

Somma, A. (2006), Il codice civile francese come simbolo, in: Dölemeyer, B., H. Mohnhaupt, A. Somma (a cura di), Richterliche Anwendung und Umsetzung des Code civil in seinen europäischen Geltungsbereichen außerhalb Frankreichs, Frankfurt M., 371-391

Somma, A. (2009), Dal lavoratore al consumatore. Cittadinanza e paradigma giuslavoristico nell'economia sociale di mercato, in: Balandi, G., G. Cazzetta (a cura di), Diritto e lavoro nell'Italia repubblicana, Milano

Somma, A. (2011a), Democrazia economica e diritto privato. Contributo alla riflessione sui beni comuni, in: Materiali per una storia della cultura giuridica 41

Somma, A. (2011b), L'economia sociale di mercato (3). L'ordoliberalismo al crollo del fascismo, in: Biblioteca della libertà 200

Somma, A. (2012), Tradizione giuridica occidentale e modernizzazione latinoamericana. Petrolio, democrazia e capitalismo nell'esperienze venezuelana, in: Rechtsgeschichte 20, 190-207

Somma, A. (2014a), Legal Change and Sovereign Debt Crisis. The Clash Between Capitalism and Democracy in the Western Legal Tradition, in: Paulus, C. (a cura di), A Debt Restructuring Mechanism for Sovereigns: Do We Need an Orderly Work-Out Scheme for Insolvent Sovereigns?, München 153-171

Somma, A. (2014b), Introduzione al diritto comparato, Roma, Bari 
TARDE, G. (1900), Le droit comparé et la sociologie, in: Bulletin mensuel de la Société de législation comparée 32

Tripiccione, A. (1961), La comparazione giuridica, Padova

Trubek, D. M., M. Galanter (1974), Scholars in Self-Estrangement, in: Wisconsin Law Review 20

Twining, W. (2005), Social Science and Diffusion of Law, in: Journal of Law and Society 32

Uslar Pietri, A. (1936), Sembrar el petróleo, in: Ahora del 14 luglio

VAlladão, H. (1954), Le droit latino-américain, Paris

Vanderlinden, J. (1995), Comparer les droits, Diegem

Wallerstein, I. (2004), World-Systems Analysis: An Introduction, Durham

Watson, A. (1993), Legal Transplants, 2. ed., Athens e London

Watson, A. (2001), The Evolution of Western Private Law, Baltimore, London

Weber, M. (2000), Economia e società (1922), Vol. 3, Torino

Wehler, H.-U. (1998), Politik in der Geschichte, München

Wolkmer, A. C. (2009), Voce South and Central America: Legal Pluralism, in: Oxford International Encyclopedia of Legal History, vol. 5

Xanbthaki, A. (2007), Indigenous Rights and United Nations Standards, Cambridge

Ziskind, D. (1984), Labor Law in Latin American Constitutions, in: Comparative Labor Law and Policy Journal 6

Zweigert, K., H. Kötz (1996), Einführung in die Rechtsvergleichung, 3. ed., Tübingen 
Marcelo Neves

\title{
Ideas in Another Place?
}

\author{
Liberal Constitution and the Codification of Private Law \\ at the Turn of the $19^{\text {th }}$ Century in Brazil*
}

\section{Introduction}

The debate concerning the introduction of liberal legal-political conceptions in Brazil is historically concentrated on the divergence between two basic views: one points to a detachment from cultural authenticity through the absorption of foreign elements which deny Brazil's - or the Brazilian Nation's - identity, singularities or peculiarities; the other suggests a deficiency, a flaw in our capacity to implement liberal values, superior in terms of civilisation and to be followed as models. Beyond the sphere of politics and law, the first orientation finds a literary expression in a famous passage by Machado de Assis: "The real country is good, it reveals the best instincts; but the official country is caricaturaland burlesque." ${ }^{\mathbf{1}} \mathrm{He}$ added: "There are certain political

* Translated from Portuguese to English by Adriano Gomes with a technical revision by Carolina Lemos and Luiz Cláudio Pinto.

1 Machado De Assis (1955 [1861]) 104. I do not find it adequate, therefore, to relate the origin of this opposition between a "real country" and an "official country", also expressed in the search for "the profound" identity of Brazil, to the artistic and literary movement that was manifested in the Modern Art Week, which took place in São Paulo in 1922, with an emphasis on Mário de Andrade, and to associate it with the adoption of democratic-social, socialist or fascist ideas in the 1920s and 1930s in Brazil, as suggested by Lopes and GARCIA Neto (2009) 27. Likewise, it would be inappropriate to associate the similar reference to the "distance between legal and real country" with the emergence of these ideas at that time, and, especially, with the difference in American realism between "law in books" and "law in action" (Lopes and Garcia Neto [2009] 15 e 21-22 - these authors eliminated this reference to American realism in the definitive Spanish edition of that article; see Lopes and GARCIA Neto [2011] 125-126). It is, as expressed by Machado de Assis, a debate founded in the $19^{\text {th }}$ century. See also note 3 . 
fortunes in our land that cannot be explained". 2 The opposing orientation is expressed in a statement by Tobias Barreto in which he compared the experience of the moderating power, established by the Constitution of 1824, with English parliamentarism: "The institutions that are not born of custom, but are an abstract product of reason, cannot stand the trial of experience for very long, and soon find themselves broken before the facts. Indubitably, our government is in such a state". ${ }^{3} \mathrm{He}$ added: "But it is important not to forget that the complicity of the people plays a role in the production of our misfortunes" ${ }^{4}$ These two ways of considering the relationship between "real country" and "official country" or, from the political-legal point of view, dealing with the presence of liberal ideas and institutions of European origin in Brazil are diverse expressions of a selfunderstanding which was suggestively called "misplaced ideas".

2 Machado de Assis (1955 [1861]) 105. This excerpt suggests that what is placed out of the system of science was not the "real country" but the "official country", unlike what can be inferred from Schwarz (2000 [1977]) 11 [Engl. transl. (1992) 19] when he sustains that the argument of a liberal pamphlet, which was contemporary to Machado de Assis, "places Brazil outside the system of science".

3 Barreto (2000 [1871]) 383. In this context, he criticises the idea that the neutral power (according to the notion of "real power" in the formulation by Constant (1957 [1815]) 1078 ff., (1872 [1914-1918]) 177 ff. would have passed "rapidly from the books to the facts": "The simple copy of a theoretical principle in an article of the Constitution does not mean that an idea has become reality. This is simply copying from one book to another book, without losing its state of pure theory", Barreto (2000 [1871]) 395.

4 Barreto (2000 [1871]) 383. The affirmation of Sílvio Romero is associated with this formulation based on the positivism and evolutionism in vogue at the time: "It is certain that the primitive inhabitants of the country have not overcome the final steps of savagery; it is also sure that our current civilisation is impregnated with barbarism. Only foolish patriots would challenge this", Romero (1960 [1888]) 83. However, Barreto had reservations in his negative assessment of the Brazilian political experience: "It is clear that is not only the good side, but also the bad side of the English government, indispensable to the conservation and harmony of the whole, that cannot be transmitted to any other country", BarRETo (2000 [1871]) 417.

5 Schwarz (2000 [1977] [Engl. transl. 1992]). Although, in a posterior essay, Schwarz (2012) seeks to move away from this self-comprehension, his famed text is ambivalent in this respect, for he himself uses the expression "inadequacy in our thinking” (2000 [1997]) 13 [Engl. transl. (1992) 20], highlighting that "our improper discourse was hollow even when used properly" (21 [Engl. transl. 25]), to conclude: "In the process of reproducing its social order, Brazil unceasingly affirms and reafirms European ideas, always improperly" (29 [Engl. transl. 29]). I will return to this issue in the final observations. 
Underlying this debate is the conception that Brazilian society has a particular identity, which distinguishes it from the European societies. From this conception derives the constant search for the peculiarity, singularity or authenticity of Brazil. In this context, the notion of society is linked to the politico-cultural concept of national state, involving territoriality itself. "Nation" as a cultural concept plays in the romantic tradition of the $19^{\text {th }}$ century a decisive role. The Brazilian nation is presented as a cultural expression of a particular society, whereas the state is understood as a political manifestation of the nation. From this results a semantics and structure proper to the Brazilian society, which would allow its comprehension and explanation. It is in this sense that the label "interpreters of Brazil" ${ }^{6}$ was conceived.

I assume, however, the theoretical supposition that modern society emerges as a world society. ${ }^{7}$ Unlike pre-modern societies, territorially delimited formations, the mundialisation of society has developed itself from the $16^{\text {th }}$ century on, was intensified during the $19^{\text {th }}$ century and was consolidated at the end of the $20^{\text {th }}$ century with the affirmation, including in the semantic sphere of self-description, of world society through the discourse of globalisation. ${ }^{8}$ Even though the economic system was originally the propellant of the emergence of world society, it is not only a characteristic of capitalism or the economic system. ${ }^{9}$ A main characteristic of world society is that the horizon of communications and expectations becomes, primarily, global, not limiting itself to a determined territory.

This is why the relationship between semantics and structure should be considered, primarily, from the point of view of the world society. Considering semantics as "a socially available sense that is generalised on a higher level and relatively independent from specific situations" ${ }^{10}$ one may inquire how semantic constructions could assert themselves as self-descriptions of world society taking into account the presence, in this society, of such distinct

6 For an overview, see Santiago (2002). In this theoretical context, talking about Brazilian society and discussing good and evil societies is still common (see, e.g. Villas Bôas Filho [2009] 337).

7 Neves (2009) 26 ff.; (2008) 215 ff. See Luhmann (1975), (1997) 145-171.

8 Neves (2009) 27-28. See Luhmann (1997) 148; Brunkhorst (1999) 374.

9 See Luhmann (1997) 158-159, with objections to the concept of capitalism as a "world system" as proposed by WaLLERSTEIN (2006).

10 Luhmann (1980) 19. 
situations on the structural plan (socially stabilised expectations). Firstly, it is important to observe that "ideas cannot arbitrarily vary in relation to the society that makes use of them. Therefore, the theoretical problem shifts to the question of establishing through what and in what way the structure of society limits arbitrariness." ${ }^{11}$ Secondly, one should underline that, considering complexity and differentiation as fundamental to the connection between structure and semantics, the "particular and general relationships between the structure of society and semantics walk, therefore, side by side and influence one another reciprocally." ${ }^{12}$ This does not prevent semantic artefacts from becoming obsolete when confronted with new emerging structures. ${ }^{\mathbf{1 3}}$ However, it is undeniable not only that semantic innovations result from structural transformation, but also that a new semanticsstimulates changes in the social structure. The matter gets more complicated when this relationship is considered at the level of world society. A semantic artefact of global society may change in view of its adjustment to the reproduction of structures in diverse social contexts. Moreover, it is important to distinguish between semantics which refers to cognitive structures and semantics which refers to normative structures.

Because the cognitive structures of economics, technique and science do not segmentally differ in the sphere of world society, the predominant semantics in these domains has the potential of presenting itself emphatically on a global level, being of little importance the structural regional differences. Therefore, the local alternative semantics is widely neutralised, since it remains subordinated to the semantics of global society: self-description of production, circulation, market, competitiveness, efficiency etc. However, with regard to the normative structures of law and politics, territorial segmentation into states rises not only the question of the confrontation between world semantics and such varied structures, but also the problem of semantic artefacts which refer to normative structures that are reproduced in the respective state.

Within the world semantics of liberalism there is an aspect related to cognitive structures which serves the self-description of the capitalist econo-

11 Luhmann (1980) 17.

12 Luhmann (1980) 34.

13 "If the level of complexity changes, the guiding semantics of experiencing and acting must adapt to it, otherwise it loses the connection to reality." LuHmanN (1980) 22. 
my, with a strong tendency to neutralise semantic alternatives. By its turn, liberalism as legal-political semantics has a strong normative dimension. This means that, during the $19^{\text {th }}$ and $20^{\text {th }}$ centuries, the liberal semantics of world society not only was submitted to tests of adequacy in light of normative structures of the states in which they were adopted, but were also pervaded by local semantics which subverted, to a large extent, its original meanings and functions. Certain shifts transmute ideas. Based on these suppositions, I shall analyse the liberal semantics that refers to the constitutionalism and codification of private law in the Brazilian experience at the turn of the $19^{\text {th }}$ century to the $20^{\text {th }}$ century. Instead of "misplaced ideas", would it not be important to inquire into the meaning and function of the development of ideas in another place or, better still, in various places of the global society? Would society in Brazil at the turn of the $19^{\text {th }}$ century to the $20^{\text {th }}$ century - delimited by the state as a territorial legal-political organisation - not be one of these places in which liberal ideas not only radiated themselves as pertaining to the dominant or hegemonic semantics of world society in relation to the normative structures, but were also confronted with local anti-liberal semantics?

In the following exposition, I shall initially analyse the factors which obstructed the unified codification of private law or the codification of civil law during the $19^{\text {th }}$ century, despite its explicit provision in the imperial Constitution of 1824 (item 2). In the second part, I will consider the limits of the normative concretisation of the republican Constitution of 1891 in view of the dominant legal-political structures and practices in Brazil during the First Republic, indicating its symbolic significance (item 3). Subsequently, I shall conduct a brief evaluation of the meaning and function of the civil codification accomplished in 1916 in the Brazilian context during the first two decades of the $20^{\text {th }}$ century (item 4). Lastly, in my final remarks, I will consider that the adoption of the social, socialist or fascist ideas in the 1920s and 1930s does not alter substantially the scenario of a semantic shift of ideas in the Brazilian legal-political experience, a situation that, to a certain extent, lasts until today. I shall also emphasise the asymmetry in the circulation of liberal ideas within the world society, as well as the paradox between the locality and worldliness of their meaning and functions (item 5). 
2 The absence of a unified codification of private law or codification of civil law in Brazil in the $19^{\text {th }}$ century

After the declaration of independence in 1822, the Law of 20 October 1823, issued by the Constituent Assembly, maintained in effect the "Filipinas" Ordinances, laws, permits, decrees and resolutions promulgated by the Kings of Portugal before 25 April 1821, while a new code was not enacted. ${ }^{14}$ With the dissolution of the Constituent Assembly in November 1823, the Emperor proclaimed the imperial Constitution of $1824,{ }^{15}$ in which the article 179, item XVIII, established that a civil code should be organised as soon as possible. ${ }^{16}$ Despite many efforts to implement this constitutional provision, it was not accomplished during the imperial period.

The "Filipinas" Ordinances - issued in 1603, when Portugal was under Spanish rule, and confirmed by the Law of 29 January 1643 - remained in effect during the entire monarchical period and lived on, underthe terms of article 83 of the Constitution of $1891,{ }^{17}$ during the first 25 years of the republican regime until 31 December 1916, completing 314 years of validity in Brazil. ${ }^{18}$ Its anachronism lied in the attribution of "extrinsic authority to the opinions of Accursio and Bartolo at a time when they were already discredited"19 In its application, arguments of authority were practically dominant and judges were satisfied with "attaching to their decisions a long procession of authors, not only lawyers, but also moralists and casuists, which at the time constituted common opinion". ${ }^{20}$ The law of 18 August 1769, issued under the regime of Marquês de Pombal, called the Law of Good Reason,

14 See Pontes de Miranda (1981 [1928]) 66; Gomes (1958) 12-13; Alves (2003) 3.

15 See Neves (1992) 116-121, with numerous bibliographic references.

16 "XVIII. It shall be put together, as soon as possible, a Civil and Criminal Code, founded in the solid bases of Justice and Equity."

17 "Article 83 - The laws of the old regime that are not explicitly or implicitly opposed to the Government systemestablished by the Constitution and the principles consecrated in it remain in effect, until revoked". In respect to this constitutional disposition, Pontes de Miranda enlightens: "Due to the implicit revoking the following institutions could no longer last, and so was understood: penalty servitude, civil death, difference between children of nobles and children of workers in the right of succession, etc."

18 Gomes (1958) 8 and 13. See Pontes de Miranda (1981 [1928]) $41 \mathrm{ff}$.

19 Gomes (1958) 9.

20 Pontes De Miranda (1981 [1928]) 43-44. This author adds (44): "Likewise, the allegations of the lawyers were reduced, largely, to accumulation, as extensive as fastidious, of remissions, almost always copied, and mostly inappropriate". 
attempted to confront this problem by offering criteria for interpreting and integrating the legal gaps and by demanding that the opinions of the magistrates were scrutinised in the light of "good reason", even establishing, in its $\mathbb{1 3}$, that Accursio and Bartolo were destituted of the authority granted by the "Filipinas" Ordinances $(\mathbb{S} 1$ of title 64 , book 3$) .^{21}$

With the changes introduced by the Law of Good Reason, the "Filipinas" Ordinances persisted until the first quarter of the Republic, at the mercy of what had been determined by the imperial Constitution (1824) itself: the elaboration of a civil code "as soon as possible". All attempts in this respect were unsuccessful.

On 15 February 1855, the jurist Teixeira de Freitas was put in charge of consolidating the legislation in effect in Brazil prior to the independence, including laws from Portugal. ${ }^{22}$ The intention was to consolidate, classify and order with the purpose of later codifying; or, in the words of Pontes de Miranda, "to know first in order to express oneself." ${ }^{23}$ In 1857, Teixeira de Freitas concluded his "Consolidation of Civil Laws", ${ }^{24}$ largely organising the "sparsest and loosest legislative elements in force at the time, which were originated from 1603 to 1857 " ${ }^{25}$ However, Teixeira de Freitas ignored slavery in his consolidation, an institution on which the economic and legal structure of the Brazilian Empire was based. In this sense, he justified his standing in the introduction of his work:

"It is important to notice that the issue of slaves is not addressed in any part of the new text. We have, it is true, slavery among us; but, if this evil is an exception, which we lament, condemned to extinction in a more or less remote time, let us make also an exception, a separate chapter, in the form of our Civil Laws; let us not stain them with shameful dispositions, not suitable for posterity; let the state of freedom remain

21 See Pontes de Miranda (1981 [1928]) 44 ff.; Gomes (1958) 9 ff.

22 Meira (1983) 94; Gomes (1958) 18.

23 Pontes de Miranda (1981 [1928]) 79. However, Pontes de Miranda's assertion should be qualified with respect to what actually came to be "consolidated" for the Consolidation also had an innovative character for legal Brazilian Empire (see Fonseca [2012] $26 \mathrm{ff}$.).

24 Freitas (2003 [1857]). After the first official edition in 1857, the second edition in 1865 and the third edition in 1876, the fourth edition was published, with the Additions in response to criticism, in 1877; in 1915, the fifth edition was published with the new laws and decrees enacted until 1913 (see Mártinez Paz [1927] XXIX). For the analysis of reach and content of the Consolidation, see MeIra (1983) $111 \mathrm{ff}$.

Pontes de Miranda (1981 [1928]) 80. 
without its odious correlate. The laws concerning slavery (which are not many) will be classified separately and will form our Black Code". ${ }^{\mathbf{2 6}}$

This formulation is in itself a symptom of the problematic coexistence with liberal legal ideas in the context of imperial Brazil. The modern notion of civil law as a normative expression of private autonomy is exposed to a disgraceful situation. The liberal jurist, in view of the slavery relations, seeks to transfer the "shameful" issue to another normative statute, the "Black Code", which was reduced to a temporary ("odious") exception, since it was not "suitable for posterity". In an effort to affirm the liberal coherence of the Consolidation by resorting to the anti-liberal exception, Teixeira de Freitas intended not only to maintain the semantics of legal liberalism - which referred to normative structures - in consonance withthe economic structures of world society at the time, but also to adjust it to the local semantics regarding the structures of normative expectations which supported slavery. The ideal of legal liberalism was, in this manner, pervaded and delimited by a slavery regime which was in accordancewith the structures of cognitive (economical) expectations of the world society and fortified by the semantics of identity concerning the normative structures of the Brazilian monarchic state. Ideas roamed, migrated, with no commitment to their eventual topos of origin.

The difficulty faced by Teixeira de Freitas was even greater because the constitutional text of 1824 , as the formal basic normative structure of the Brazilian empire, implicitly predicted slavery. Although this nuance was not taken into account, leading to the belief that - contrary to the project of the 1823 Constituent Assembly - the Constitution of 1824 did not predict slavery, it did indirectly recognise the slavery regime by distinguishing, in article 6, item I, between freeborn [ingênuos] and freed [libertos] citizens. This distinction is only meaningful in slave orders, for the condition of being freed presumes a prior situation of slavery in contrast to those who were born free. $^{27}$

It was in this context that, on 10 January 1859 , Teixeira de Freitas was hired by the imperial government in order to elaborate a draft of a civil code. ${ }^{28}$ This

26 Freitas (2003 [1857]) vol. I, XXXVII. See Surgik (1988); Meira (1983) 113-114; MercaDANTE (1980) 188.

27 Oddly, in their comments about article 6, item I of the imperial Constitution, neither Bueno ([1857] 450-453) nor Sousa ([1867] 40-53) nor RodRigues [(1863] 10) made any reference to this issue.

28 Nabuco (1997 [1897-1899]) 1053; Meira (1983) 185; Pontes de Miranda (1981 [1928]) 80. 
time, his task was not to elaborate an artefact of systematic self-description of the dominant normative structures, but to get directly involved in the reform and transformations of these structures, at least in their textual formal aspect. ${ }^{29}$ The draft was not concluded, not having been completed the third book of the special part, which referred to common provisions relating to rights "in persona" and rights "in rem". ${ }^{30}$ Finally, in 1872, the contract for the elaboration of the project was revoked because the government did not accept the idea defended by Freitas of elaborating a general code of private law. ${ }^{31}$ In a proposal sent to the government in 1867 , Freitas stated the following:

"The government wants a Project of Civil Code to function as a subvention to the complement of the Commercial Code; it aims to preserve the existing Commercial Code with its intended revision, and today my ideas are different, unwaveringly resisting to this calamitous duplication of Civil Laws, not distinguishing, within the entirety of the laws of this class, a branch that demands a commercial code. The government only wants from me the writing of a Project for a Civil Code, and I cannot deliver this project, even if it comprises what is called Commercial Law, without starting with another code, which encompasses the whole legislation." ${ }^{32}$

Note that the Brazilian Commercial Code had been promulgated in 1850 . Teixeira de Freitas claimed that it commercialised all civil relationships. ${ }^{33}$ The relative ease with which the Commercial Code was approved contrasted with the obstacles that the government and the parliament imposed in relation to the Draft. ${ }^{34}$ In this matter, the question of slavery is once again stressed. The Commercial Code was not dissonant with the slavery regime because the rural landlordswere involved in the relations of production and circulation of the global economy and needed a modern market regulation:

29 See Nabuco (1997 [1897-1899]) 1054.

30 Pontes de Miranda (1981 [1928]) 81; Gomes (1958) 19. See Nabuco (1997 [1897-1899]) 1054 ff.; Meira (1983) 186 ff. The Draft (Freitas [1983]) was originally published in 1864.

31 Nabuco (1997 [1897-1899]) 1062; Meira (1983) 361-362; Gomes (1958) 19, nota 18. However, the Justice Section of the State Council accepted the proposal, Nabuco (1997 [1897-1899]) 1060-1061; Mártinez Paz (1927) XIX; Meira (1983) 356-358; Alves (2003) 4.

32 Apud Nabuco (1997 [1987-1989]) 1057. The letter can be found in its entirety in MeIra (1983) 352-356.

33 Freitas (1878) XI-XII. See Mercadante (1980) 184.

34 See Mercadante (1980) $189 \mathrm{ff}$. 
"Within the scope of the private law, the rural landlord [...] could not do without a body of liberal laws that would regulate his relations as a seller with the market, where he placed, as a trader, what abounded from the production of his farm. In this field, his interests coincided with those of the exporting commerce of the port-cities. They were tied to exporters and commissioners or even to small traders in a complex of social, commercial and legal relations." ${ }^{35}$

Teixeira de Freitas reacted against the duality of private law because he was contrary to the "duplicity in our relations of production". ${ }^{36}$ His "ingenious" escape from the matter of slavery, by proposing a "Black Code" apart from the Consolidation of Civil Laws, could no longer be a solution because, in thisnew context, the issue was the elaboration of the project of "a Code in consonance with legal individualism". ${ }^{37}$ The Consolidation served to maintain the existing normative structures, whereas the Draft would serve a codification based on liberal individualism. The former could coexist with the slave regime, but the latter was exposed to a paradox: the individualist codification of private law liberal and universal under its semantic self-description - supposed overcoming the slavery regime or, at least, if approved, it would call this regime into question. In this sense, the Consolidation, in conformity with the social structures of slavery, was a decisive factor for the Draft not having been adopted, revealing an evident "incompatibility between legal individualism and the objective conditions of the Brazilian economic reality". 38

In this regard, it is noteworthy that although it was not adopted in Brazil, the Draft had a great influence on the legislation of other countries, such as Argentina, Uruguay, Paraguay and Chile. ${ }^{39}$ In these countries, however,

35 Mercadante (1980) 184.

36 Mercadante (1980) 190.

37 Mercadante (1980) 188.

38 See Mercadante (1980) 191.

39 Pontes de Miranda (1981 [1928]) 63; Mercadante (1980) 194. About the repercussions of the work of Teixeira de Freitas in other countries (including European ones), see MeIrA (1983) 387 ff. In Argentina, Dalmacio Vélez Sársfield, when presenting the first book of the Project of the Civil Code to the Minister of Justice, on 21 June 1865, declared that he had consulted many foreign codes "and, above all, the Project of the Civil Code that is being made for Brazil by Mr. Freitas, from whom many articles were taken", apud Meira (1983) 288; see Mercadante (1980) 194, note 24; Pontes de Miranda (1981 [1928]) 80. In his turn, Senator Carlos Serrey declared that the Argentinian Civil Code "Constitutes a matter of honor for doctor Vélez Sársfield and for the Brazilian jurisconsult Freitas, his main guide" (apud Pontes de Miranda, ibidem). In this respect, see, in detail, Meira (1983) 267 ff.; Mártinez Paz (1927), especially XXXII-XXXIII and XLV-XLIX; LeVAGgi (1988). 
slavery had already been abolished, enabling the adoption of the individualist codification of private law, which was evidently adapted to the local variables.

These considerations about the failure of the civil or unified codification of private law in the Brazilian empire, ${ }^{\mathbf{4 0}}$ which maintained slavery until its decline, contain evidences which could reveal the very limits of displacing ideas to diverse legal-political contexts. Without a doubt, legal liberalism implies ideas that are constructions belonging to the semantics of world society in the $19^{\text {th }}$ century. In this domain, although permeated by normative semantics of the national state's identity, these constructions circulated with their particularities in the political and legal discourse of the Brazilian Empire. However, because they intended to be enforced and to have an institutional form as a structural artefact with a normative character in the local sphere, they were exposed to almost insurmountable obstacles: the slavery regime, pertaining to the economic structure of the world society in the $19^{\text {th }}$ century and legitimised by the local normative semantics and structure of authenticity, made unlikely the institutionalisation of individual liberal ideas through the codification of civil law. Therefore, in these circumstances, in terms of structure, "the revocation of the Ordinances would only be put into effect with the end of the slavery regime"."

However, this statement should not be interpreted in terms of an excluding determinism between civil codification and slavery. For instance, the Louisiana Civil Code provided for slavery. The assertion should be understood within the limits of the Brazilian context and the intent of Teixeira de Freitas to remain faithful to the liberal legal ideas. ${ }^{42}$ Moreover, it would be

40 In addition to the Draft from Teixeira de Freitas, two other attempts of civil codification in the Brazilian Empire were made, also unsuccessful: the projects by Nabuco de Araújo, see Nabuco (1997 [1897-1899]) 1062-1074), and Felício dos Santos, see Pontes de Miranda (1981 [1928]) 82.

41 Mercadante (1980) 194.

42 See Fonseca (2011) 24-25. It should by no means be forgotten that the liberal economic semantics of world society interpenetrated with the slaveholding ideology of the Brazilian Empire, and in this regard there are no grounds for rejecting Bosi's remark (1971, 195) that: "The formally dissonant pair slavery-liberalism was a mere verbal paradox, at least in the Brazilian case." However, as argued in the Introduction, it is important to distinguish between the "factual" semantics of liberalism, grounded in the structures (cognitive expectations) of world capitalism, and the "counterfactual" semantics of liberal legal idealism, on which the Esboço was based to a certain extent and which was directly linked to political and legal structures (normative expectations). As for this latter semantics, it is possible to discern 
more appropriate to consider not only slavery as an obstacle to liberal civil codification in Brazil during the $19^{\text {th }}$ century, but also the disputes surrounding the meaning of citizenship in general. ${ }^{43}$ One might add that the Consolidation - by practically assuming the role of a civil codification - was more suitable and "functional" to the structure of legal and social exclusion in the Brazilian Empire than a civil or private code with a strong liberal tendency, which could put in jeopardy the maintenance of the socio-political and legal-economic framework of the monarchist regime.

However, from the semantics perspective, the liberal individualist ideas on private law were circulating with functions and meanings that differed from those of the places in which they were originated, not only as politicalsymbolic artefacts of "ideological" self-descriptions of Brazilian social and legal reality, but also as reflexive and critical elements of the form of integration of the Brazilian state in world society. In this particular context, the paradox between global and local conditioned and stimulated the migration of liberal ideas concerning the codification of private law in Brazil during the $19^{\text {th }}$ century.

\section{From the liberal Constitution of $1891 \ldots$}

After the abolition of slavery in 1888 , which was one of the economic and social foundations of the monarchy, and in the context of the conflicts between the government and military and religious orders, the Republic was proclaimed in 1889 in a very singular manner, through a military coup, which was rather unexpected and incomprehensible to the populace. ${ }^{\mathbf{4 4}}$

the existence of a paradoxical tension between the liberalism of the Esboço and the slaveowning ideology of the Empire. On this matter, Bosi himself admits (ibidem) that the content of the pair "slavery-liberalism" could be considered a "real contradiction if the concrete content of the second term, liberalism, were fully equivalent to the bourgeois ideology of free labour affirmed throughout the European industrial revolution". However, Bosi did not admit the differences which I take as my starting-point and which entail paradoxes, because he espoused a dialectical ontology of totality (hence his preference for speaking of a "contradiction").

43 Grinberg (2002), especially 319-321; Grinberg (2008) 47 ss.

44 According to the famed formulation from Aristides Lobo: "The people watched the event in a bemused, amazed, surprisedstate, without realizing what it meant. Many believed it to bea parade" (apud Maximiliano [1948] 105). 
Summoned the Constituent Assembly (1890-1891), ${ }^{45}$ Rui Barbosa, fascinated by the constitutional experience of the United States, offered a draft in which were emphasised liberalism, presidentialism and federalism. Although the project underwent some changes in the Assembly and, to a certain extent, the final documentdetached itself from North American constitutionalism, these features characterised the Constitution of 1891.

Firstly, it is important to observe that the constitutional ideas adopted in the normative textual structures conflicted, at the level of constitutional practice, with Comtean positivism which influenced the ruling military elite in the first years of the Republic, since it was understood from a perspective which allowed the justification of any violation of constitutional norms in the name of defending the "order" ${ }^{46}$ This confrontation between the liberal ideas took shape in the constitutional text and the authoritarian ideas of the constitutional practice conducted by the military executive during the first years of the Republic is an evidence of the incongruity and complementarity between constitutional textualising and implementation.

With the Constitution of 1891, the relationship between constitutional text and the reality of the power process became even more problematic if compared with the constitutional experience of the Empire. ${ }^{47}$ The declaration of individual rights and freedoms was expanded, and the principle of the "separation of powers" was affirmed in the constitutional document, but the subjacent social structure and the dominant political practices were reproduced, to a great extent, beyond the scope of the textual-normative contour of the Constitution. The permanent distortion or violation of the Constitution throughout the period during which it was in effect (1891-1930) ${ }^{48}$ can be regarded as the most important aspect of the legal-political reality in the first Republic. What constitute significant expressions of the lack of normative

45 For a detailed exposition of the sessions of the Constituent and respectivediscussions, strongly imbued with American constitutionalism, see Roure (1918-1920). See note 57.

46 On the distortion of Auguste Comte's positivism at the time in Brazil, see BuARQue DE Holanda (1985) 289-305.

47 Although in another perspective (in the search for the identity of the "Brazilian society"), Buarque de Holanda (1988) 125 was aware of this issue and noted that, with the implementation of the Republic, the State "was uprooted" even more from the country. According to Faoro (1976) 64 "arbitrariness" was fortified. See also Carvalho (1996), espec. 379.

48 See Pacheco (1958) $240 \mathrm{ff}$. 
concretisation of the constitutional text are: electoral fraud as a rule in the political game controlled by the local "oligarchies"; ${ }^{49}$ the degeneration of presidentialism in the so-called "neo-presidentialism", ${ }^{50}$ primarily through excessive declarations of martial law: ${ }^{51}$ the deformation of federalism through the "governor's politics" 52 and the abusive proclamation of federal intervention in member states. ${ }^{53}$

Among the conservative critics, supporters of an authoritarian, corporative and nationalist state, the problem of the lack of normative concretisation of the constitutional text of 1891 was denounced as a contradiction between "constitutional idealism" and "national reality". ${ }^{54}$ However, in their criticism of the "utopian idealism" of the constituent legislator, the symbolic significance of the constitutional document was not accurately considered; on the contrary, the accent was on the ingenuity of its "good intentions". ${ }^{55}$ In these terms, the Constitution would be an expression of misplaced ideas. The question whether the so-called "utopian idealism" was only adopted in the constitutional document insofar that the realisation of the respective principles was postponed to a remote future and, thus, the status quo was not threatened, was not part of the discussion. Furthermore, one cannot forget that the "nominalist Constitution" of 1891 functioned as an artefact of symbolic identification of the Brazilian legal-political experience with the North American (US) one, creating an image of a Brazilian state as "demo-

49 In this respect, see Neves (1992) 170-171. Clearly, the electoral problems were not reduced to constitutional practice because the lack of guarantee of secret vote fostered electoral fraud and the inexistence of voting rightsto illiterates and beggars (article 70, $₫ 1$ o, clauses 1 and 2, of the Constitution) excluded from the electoral process a large portion of the population according to official data (IBGE [1989] 72), 65.1\% of the population over 15 years of age were illiterate in 1900 and $64.9 \%$ in 1920. In addition, women were banned from voting until the electoral reform of 1932 (Decree 20,076, Feb 24, 1932) after the fall of the first Republic ad the revoking of the Constitution of 1891 . From 1898 to 1926, the electoral participation oscillated between $3.4 \%$ and $2.3 \%$ of the population, according to FAORO (1985 [1958]) $620-621$.

50 On this concept, see LoEWENSTEIN (1975) 62-66.

51 See Barbosa (1933), vol. II, 373 ff.; 1933, vol. III, 323 ff.

52 On the so-called "governor's politics", see, e.g., Faoro (1985 [1958]) 563 ff.; Carone (1969) 103 ff.; Carone (1971) 177 ff. Cardoso (1985) $47 \mathrm{ff}$. calls it an "oligarchic pact".

53 See Barbosa (1934) 17.

54 In this sense, see primarily Vianna (1939) 77 ff.; Torres (1978 [1914]).

55 See, e.g., Vianna (1939) 81, 91 and 111.

$60 \quad$ Marcelo Neves 
cratic" and "constitutional" as its model. At the very least, the rhetorical invocation of liberal and democratic values that were consecrated in the constitutional document worked to discharge the "owners of power", transferring to the supposedly "backward society" the "responsibility" or "blame" for the disrespect for the Constitution.

Nevertheless, the question should not be put in terms of an alternative between liberal idealism (illuminist, alienated) and authoritarian realism (authentic, deeply rooted). The ideas of liberal constitutionalism circulated among the civil sectors of an emerging middle class, which remained confined between privileged minorities tied primarily to rural estates and "subaltern" or excluded masses in the rural areas and cities. Rui Barbosa is a typical expression of this new middle class. ${ }^{56} \mathrm{It}$ is undeniable that there was a rhetorical exaltation of the American Constitution (such as occurred with French constitutionalism and English parliamentarism during the Empire) which was capable of cultivating the symbolic illusion that the simple transplantation of the Constitutional model of the United States would offer an adequate solution to Brazilian social problems. ${ }^{57}$ In this sense, Oliveira Vianna spoke of the "belief in the transfiguring power of written formulas" ${ }^{58}$ Notwithstanding, even Barbosa was not absolutely uncritical of the possibility of transferring North American institutions to Brazilian reality through the promulgation of the Constitution or legislation. ${ }^{59}$ The issue lies in the fact that, in terms of the world society semantics at the turn of the $19^{\text {th }}$ to the $20^{\text {th }}$ century, the constitutional ideas circulated and migrated without a clear commitment to their origin. Barbosa was a jurist involved in this discussion at the time. The problem that is posed refers to the moment when these ideas were intended to take shape at the structural level of the constitutional norms, and, therefore, to influence the stabilisation of normative expectations fundamental to the life of states in such diverse social contexts. In truth, the comprehension of this question requires that the debate goes beyond the dichotomy between "idealism" (alienating, foreign)

56 Dantas (1962b), espec. 24, 27 and 36-38.

57 "But the sweetheart at the time was North-American presidentialism, which rapidly provoked the progress of the great friendly nation, as parliamentarism had provoked England's" (Roure [1920] 354.

58 Vianna (1939) 91; see also 81.

59 See Barbosa (1932) 30. 
and "realism" (authentic, national) or between "real country" and "official country". It is only possible to understand the legal-political reality of the country ascomposed by "idealism" and "realism" in a relationship of both tension and complementarity. Thus, one must consider the dynamics of the dislocation of ideas (on the semantic level) in view of the normative structures and the operations of the legal-political practice.

The semantics of the liberal constitutionalism, articulated according to the terms of the North American experience, was assimilated in the sphere of normative structures by means of the constitutional formalisation of 1891 . However, this semantics was intersected by the local semantics of authenticity, which later found structural expression in the authoritarian Constitution of 1937. Moreover, constitutional textualisation is a tenuous dimension and by itself is of little significance to the normative-legal structures. The normative expectations were pervaded by criteria of over-inclusion and under-inclusion in the legal and political systems. ${ }^{60}$ A privileged minority acted outside the Constitution limits and the respective sanctioning mechanisms did not have any relevant practical meaning. The actions of the governmental bodies or agencies were not excluded from this situation. In this sense, Barbosa himself was emphatic in his comments on the Constitution:

"One of the scourges which disgrace the country today are the so-called local state oligarchies, which are incited, explored, sustained and aggravated by the Federal Government, using, in order to do so, the sea and land military forces, and the civil army, which our innumerable civil servants offer".61

This practice of the over-included, located, so to speak, "above" the Constitution, was inseparable, during the first Republic, from the situation of the under-included or excluded from "below", who were beneath the Constitution. These were already excluded by the very constitutional provisions, which established that the illiterate and beggars, the major parte of the population, could not vote. Furthermore, beyond these constitutional provisions, the social exclusion of large segments of individuals and groups made the declaration of individual rights and guarantees irrelevant to them: depending

60 On the sub-inclusion (or sub-integration) and over-inclusion (or over-integration), see NevES (2005); Neves (1992) 94 ff. and 155 ff.; NeVes (2006) $261 \mathrm{ff}$.

61 Barbosa (1934) 17.

62 Marcelo Neves 
on the urgent satisfaction of vital needs, most of them formally freed from slavery, lived as subordinates to masters and bosses or as socially insignificant bodies. $^{62}$

The relations of over-inclusion and under-inclusion were a factor of obstruction in the constitutional concretisation and applicability. The Constitution was not concretised in a generalised way, but in a selective and particular manner, in accordance with the concrete constellation of interests of the over-included. It did not work primarily as a legal horizon for those who held the power, but was used, abused and unused in accordance with factual and casuistic conditions of the political relations of domination. In other words, the constitutional concretisation was strongly conditioned and limited by the arbitrary injunctions of the "owners of power". In this way, the Constitution imploded as a fundamental reflexive dimension of legal-political practices.

Would this mean that the ideas of individual liberal constitutionalism, at that moment inspired mainly by the experience of North American presidentialism and federalism, were "misplaced ideas"? In the Brazilian state of the first Republic (1889-1930), a territorially delimited legal-political topos, these ideas suffered a shift of meaning due to the relationship between politics and law as precariously differentiated functional systems. From a strictly legal-normative point of view, its constitutional textualising had little structural significance for the generalised stabilisation of normative expectations in terms of an adequate constitutional concretisation. This structural matter, by its turn, is inseparable from the operational problem, taking into account that communications and respective legal-political actions diverted from the textual model of theConstitution. However, from a political perspective, the liberal ideas incorporated to the constitutional text played a relevant yet ambivalent symbolic role. On the one hand, the text served as a certain constitutional self-illusion of the rhetorical and "ideological" identification of the Brazilian state with the constitutional experience in the United States and in the European states at the time. On the other hand, the constitutional text served the criticism made by intellectuals and the opposition with respect to the political and legal practices that violated the Constitution. In part, as previously stated, this criticism strived for the

62 Pontes de Miranda (1981 [1928]) 82-83, bearing racialist remarks. We return this issue in item 4. 
authoritarian state, against "utopian idealism" and in favour of "organic idealism" ${ }^{63}$ in accordance with the semantics of a national authenticity. But this positionalsopointed to a claim for constitutional concretisation and efficacy. Being included in this second strain of thought, Rui Barbosa, the main author of the project of the Constitution of 1891, was an expression of the ambivalence of this political-symbolic function of liberal ideas embodied in the constitutional text: his liberal rhetoric that praised the Constitution coexisted with his criticism of governmental practice. In the scope of this hypertrophied symbolic ambivalent function of the constitutional text which shaped the liberal legal-political ideas in terms of the North American experience, one did not take into account, however, the fact that the generalised legal-normative concretisation and efficacy of the Constitution required radical - not to use the term "revolutionary" - ruptures in the structure of society in Brazil during the first Republic. Evidently, for Brazil, this alternative was not in the historical horizon of possibilities of the world society at the time, a world society founded on a strong economic and social asymmetry between countries and regions. ... To the Civil Code of 1916

There is a close connection between the liberal Constitution of 1891 and the civil codification that was consolidated in 1916. The paradoxical articulation of individual liberal ideas, weak normative-legal structures and social structures with strong excluding potential develops asa process that begins with the formal constitutionalisation and solidifies with the codification.

In 1890, the provisional government put jurist Antônio Coelho Rodrigues in charge of elaborating a Project for a Civil Code which, concluded in 1893, was subsequently rejected by a commission nominated by the government and, later on, even though approved by the Senate (1896), was not pursued in the House of Representatives. ${ }^{64}$ In 1899, the government invited Clóvis Bevilaqua, then Professor of Comparative Legislation at the Law School of Recife, to elaborate a new Project for a Civil Code. Having initiated its elaboration in April, in November Bevilaqua had already concluded his work.

63 See especially Vianna (1939) 9-13.

64 Brandão (1980) 19; Pontes de Miranda (1981 [1928]) 82-83. The complete text is available in Coelho Rodrigues (1980). 
A committee constituted by the government revised the project and sent it to the House of Representatives in 1900. Having Sílvio Romero ${ }^{65}$ as the rapporteur, the Project was approved in the House of Representatives with many modifications and submitted to the Senate in 1902. Having as rapporteur Rui Barbosa, whose report, presented on 3 April 1902, focused on restrictions against linguistic and grammatical formulations without really taking into account the legal aspect of most issues, ${ }^{\mathbf{6}}$ the project remained stuck in the Senate until 1912, when the House of Representatives finally began to discuss it. In both Houses of Congress the project originally revised by the government suffered many modifications and was only approved in December 1915, being sanctioned and promulgated on 1 January 1916 and put into effect on 1 January $1917 .^{67}$

Although the code was, from a philosophical point of view, strongly influenced by evolutionism and positivism and, from a legal point of view, impregnated with the jurisprudence of interests (Ihering) and marked by a certain "legal sociology", ${ }^{68}$ Clóvis Bevilaqua proposed a code which basically corresponded to the model of liberal codification of the $19^{\text {th }}$ century, ${ }^{69}$ which followed a conceptualist tradition of law. He did not intend to present, "like Freitas, an original and revolutionary work" but to select solutions found in prior projects, in foreign codes and in the law in effect in Brazil. ${ }^{70}$ Despite its liberal nineteenth century posture, Bevilaqua's project (1899), in some fundamental points, proved to be less insensitive to the social transformations

65 His report was adopted by the Special Committee of House of Representatives on January, 18, 1902 (see Comissão Especial da Câmara dos Deputados, 1902).

66 See Bevilaqua (1906) 449-478, in response to the criticism by Rui Barbosa as expressed in his report (BArbosa [1902]) and in the famous Reply from 31 December 1902 (Barbosa [1904]), which led, respectively, to the answer and the "rejoinder" by Ribeiro (1902) 1905. On this controversy and its legal-political implications, see the pioneering study presented by Veronese (2013).

67 For a summary on the project's elaboration, its revision by the government and proceedings in the National Congress, see Pontes de Miranda (1981 [1928]) 83-86. On the involvement of Rui Barbosa, including his legal report presented in the Special Senate Committee in 1905 (Barbosa [1968]), see Dantas (1962c).

68 See Machado Neto (1969) $112 \mathrm{ff} . ;$ Dantas (1962d) $84 \mathrm{ff}$.

69 "The mental date of the Code (as with the B.G.B. and the Swiss Code) is very 1899; it would not be inadequate to claim it as the antepenultimate Code of last century [19th]", PONTES DE Miranda (1981 [1928]) 85 .

Dantas (1962d) 89. 
of the $20^{\text {th }}$ century than the Civil Code approved in 1916. In two aspects this difference can be clearly seen, namely in the social question and in the issue of family and gender.

Concerning the first aspect, the observations by Santiago Dantas are enlightening:

"Bevilaqua had not incorporated, certainly, to the primitive Project, solutions which could represent the new legal conception of labour relations, similarly to what the German Civil Code did by distinguishing the service contract from the labour contract $(\$ 631)$, but his handling of the matter was much more advanced than the one that resulted from the modifications made in the course of the revision. Amongst the norms proposed by him, and excluded from the Code, there were the right to receive salary in the case of transitory inability to work due to sickness (article 1369), or the duty of medical assistance to domestic labour (article 137), the prohibition of industrial or mine labour to those under 12 years of age (article 1381), the limitation to six hours of labour to those under the age of 16 years (article 1382), the duty of the employer to eliminate insalubrious workconditions under penalty of answering for its consequences (article 1383)., ${ }^{71}$

As these embryos of labour legislation were rejected, labour lawonly started to be developed at the beginning of the 1930s, and the Consolidation of Labour Laws of 1940 was a landmark of its establishment in Brazil, remaining long time excluded from it protection the rural and the domestic worker. ${ }^{72}$ The latter, until recently, was not covered by the regime of Government Severance Indemnity Fund for Employees, whose main role was to substitute the regime of work stability and compensation for time of service in the event of dismissal without just cause. ${ }^{73}$

Regarding the question of family and gender, although Bevilaqua was radically opposed to divorce, which he considered a retreat "from the moral standing of monogamy to the regime of successive polygamy" ${ }^{\prime 74}$ and his project was strict in defining the husband as the head of the conjugal partner-

71 Dantas (1962d) 90.

72 The changes to legally include the rural worker only started to occur when the Rural Worker Statute came into effect (Law no. 4,214, 2 March 1963), but at a very slow pace and without the corresponding practical effects (see MoraEs Filho [1982] 111-115).

73 The current restrictions to the rights of domestic workers are supported by the sole paragraph of article 7 in the Brazilian Constitution. The new Constitutional Amendment Nr. 72/2013 approximates the rights of domestic workers with all other workers' rights. However, the level of illegal informality in hiring domestic workers remains very high $(73.3 \%$ in 2009 ; see Gomes (2011).

74 Bevilaqua (1906) 98.

66 Marcelo Neves 
ship (article 272), he was against the relative incapacity of the married woman for most acts of civil life, ${ }^{75}$ the solution adopted by the Civil Code (articles 240-247 and 252) and only overcome by modifications introduced by Law no. 4,121 from 27 August $1962 .{ }^{76}$ Article 279 of Bevilaqua's project stated that, through marriage, "the woman becomes the companion and partner of the husband", in contrast with the original text of the Civil Code which stated that the woman took, through marriage, "the condition of his companion, consort and assistant in domestic affairs". Through this perspective, although he expressed the ideasof his time on the differences between man and woman, Bevilaqua, unlike the Civil Code, pointed to a solution almost in line with equality between genders according to his own words:

"Developing the same thought, seeking to meet the legitimate aspirations of women, and with the intention of making marriage an equal partnership, although under the direction of the husband, the Project conceded a larger sum of rights, more freedom of action to the married woman than the law that is currently in effect." 77

He then added, "The Project intended to recognise women as equal to men, but without deviating women from the role that nature itself bestowed upon them, rationally interpreted" ${ }^{78}$ However, he pondered: "The author of the Project is convinced that he was, in this topic, as liberal as he was allowed to be". 79

It is interesting to add that, still pertaining to family matters, Bevilaqua's Project held in better regard the legal issue of the then called "illegitimate sons" thanthe solution adopted by the Civil Code,${ }^{\mathbf{8 0}}$ distancing himself from the dominant moral and religious conceptions at the time in Brazil. ${ }^{\mathbf{8 1}}$

With reference to the dispositions concerning family and gender, jurists who were interested in thehistoricalprocess of the codification attributed to the Civil Code - even more than to the original project - anti-liberal traits or,

75 See Bevilaqua (1906) 93-96.

76 On the changes attributable to this Law, see the succinct legal exposition by Bueno (1972).

77 Bevilaqua (1906) 93-94.

78 Bevilaqua (1906) 95.

79 Bevilaqua (1906) 96.

80 See Bevilaqua (1906) 99-105.

81 On this subject, Pontes de Miranda stated, "Clóvis Bevilaqua was the most favourable to women (articles 251 and sole paragraph, 254, 287, 393, and 414, I), to the rights of illegitimate sons (article 367) and family solidarity (articles 332, 409, 416, 447 and 467)" (Pontes de Miranda (1981 [1928]) 454). 
at least, non-liberal ones. According to Pontes de Miranda, several passages within the Code "display the preponderance of the family, still patriarchal", constituting "law that was more concerned with the social sphere of the family than with the social spheres of the nation or the classes". ${ }^{2}$ This position was corroborated by Orlando Gomes, who highlighted the influence of domestic privatism in its elaboration. ${ }^{\mathbf{8 3}}$ Regarding this matter, some dispositions - concerning the indissolubility of matrimony, the universal communion of goods, the possibility to opt for a regime of separation of goods, guardianship and curatorship and hereditary rights - were pointed out, all favouring the immensely privileged position of the husband as the head of the conjugal partnership, as for instance, the unconditionalright of the testator "to mark the assets of the heirs, even those to be acquired as their legitimate part, with a lifelong inalienability clause" ${ }^{\mathbf{8 4}}$ Specifically related to the question of gender, article 236 - which prescribed as valid, without the consent of the wife, "nuptial donations made to the daughters and donations made to the sons due to the occasion of their marriage, or of the establishment of a separate household" - reflected, according to Pontes de Miranda, "the precarious situation of the Brazilian woman": "It should have included the daughter, who, not getting married, wished to establish an autonomous life, a "separate household" ${ }^{85}$

This prevalence of the family sphere was associated with legal affectionateness, tolerance and benevolence. ${ }^{86}$ The very author of the original project, Clóvis Bevilaqua, claimed that "Brazilian civil law can be considered an 'affective law' because many of its most characteristic dispositions were made due to sentimental motives", adding that "two general principles can be found as propulsive or inspiring energies for our legal life (legislation, doctrine and jurisprudence): the "feeling of freedom" and "idealist impulses" ${ }^{\mathbf{8 7}}$ This suggestion of the prevalence of a "jurisprudence of feelings" in Brazil was criticised by Pontes de Miranda due to its "despotic" elements. But he also connected it to a "legal benevolence" that was, on the one hand,

82 Pontes de Miranda (1981 [1928]) 443.

83 Gomes (1958) 21-35.

84 Gomes (1958) 24-28.

85 Pontes de Miranda (1981 [1928]) 449.

86 Pontes de Miranda (1981 [1928]) $441 \mathrm{ff}$.

87 Bevilaqua (1975 [1922]) 193. 
criticised on the basis of racialist arguments and, on the other hand, confused with "excessive liberalism". ${ }^{\mathbf{8 8}}$ In fact, expressions such as "affection" and "benevolence" were used in an ideologically simplifying manner, diverting the attention from problems regarding asymmetries, relations of dependency and exchanging of favours, and the still patriarchal dominance within the family. Moreover, even if the presence of an "affectionateness" is taken into account as a factor of rulemaking and adjudication in private law, this trait would have to be defined as somewhat opposing individualist liberalism and considered, above all, in its oppressive facet, as it was related to the arbitrary power of the socially privileged at the expense of the weakest. Therefore, instead of taking seriously expressions such as "affective law" and "legal benevolence", it would be more appropriate to relate the influence of the socalled "domestic privatism" with the persistence of a ruralframework in the first decades of the Republic which, despite the abolition of slavery, still maintained practices of exclusion, privileges and dependency relationships that were analogous to the social structure of the Empire.

Therefore, the issue of gender and family relates directly to the "social question". In this respect, Orlando Gomes emphasised that the Civil Code did not adopt anyclause concerning social rights and labour relations and overlooked changes taking place in Europe. ${ }^{\mathbf{8 9}}$ The Code had remained faithful to the legal individualism of the $19^{\text {th }}$ century. In fact, as previously stated, there was nothing in the Code regarding work accidents, protection of workers in case of sickness, prohibition of child work, limitations to the labour journey and protection of workers in insalubrious working conditions. The various

Pontes de Miranda (1981 [1928]) $444 \mathrm{ff}$. Concerning racialism, this author stated: "Legal benevolence, the exaggerated affection, which Clóvis Bevilaqua later recognised as characteristic, and showered it with compliments, constituted an energy defect, a result of the Black element mixed in the population [...]. The Black and the Indian represented biological influences, and not sociological, that is, from social facts to social facts. There are no institutions of Black or Indian law in Brazilian law; but there are Black and Indian factors in the way of being and in the legal activity of the Brazilian: these influences must be eliminated and it is beneficial that the extirpation of the inconvenience they cause is done quickly" (ibdem, 445-446). Therefore, he defended the opportunity of "eugenic measures" (447). Also in accordance with the dominant racialism at the time, BEviLAQUA (1975 [1922] 194) referred to Blacks and Indians as "affective races". On this matter, see GrINBERG (2008), especially 34-37. On Bevilaqua's “racism”, see Machado Neto (1969) 125. 
attempts presented in the debates to ensure minimum elements of social rights failed. ${ }^{90}$

According to Orlando Gomes, Clovis Bevilaqua himself, author of the original project, "assumed, in a clear and firm way, a categorical position against innovations regarding the social question". ${ }^{\text {I1 }}$ In defence of the project, Bevilaqua really showed mistrust in what he defined as "socialism", even in the sense of recognising rightsin the terms of a social state, which, according to him, could "turn into a socialism that absorbs and annihilates individual stimulus". ${ }^{2}$ He argued that, "social private law cannot be something other than the balance between the interests of individuals and society", supporting that, apart from these parameters, reforms "will be subversive". ${ }^{3}$ However, it is important to note that in the social context of the authors involved in the elaboration, the individualism that prevailed in the Code went far beyond the precautions of Bevilaqua with what "it contains of exaggeratedly selfish and disorganising." "The code had not erased, in the words of the redactor of the project, "the stain of bourgeoisie that tarnishes the prevailing civil codes"

This issue emerged primarily in the discussions about hiring of services from the Special Committee of the House of Representatives. ${ }^{\mathbf{9 6}}$ A strong conception of autonomy of will frustrated any attempt to establish a distinction between a service contract celebrated in the sphere of private autonomy and an employment agreement. Not even the most rudimentary elements of worker protection present in Bevilaqua's original project were admitted. In this "legal individualism which was hostile to all legislative labour regulation", ${ }^{97}$ Pontes de Miranda identified a law that was more concerned with "an undisguised capitalism, although innocently convinced

90 Gomes (1958) ibidem.

91 Gomes (1958) 57.

92 Bevilaqua (1906) 41. "And I speak only of this socialism that is presented as an empirical solution to the harsh contingencies of the present, and not of this genuine product of mental anarchy that charges against property, family and govern organisation, without knowing which forces will take the place of the ones it intends to eliminate" (ibidem). This second feature of socialism was defined as "ferment produced by a state of unrest, embarrassment, and revolt that torments a significant part of mankind” (42). See Gomes (1958) 43.

93 Bevilaqua (1906) 40.

94 Bevilaqua (1906) 41.

95 Bevilaqua (1906) 40.

96 See Gomes (1958) $58 \mathrm{ff}$.

97 Gomes (1958) 61. 
of its function of solidifyingsocial justice."98 In turn, according to Orlando Gomes, "the dominant mentality at the time was transfused with such objectivity into the codified rules regarding the service contract chapter, that none other could express it more clearly" ${ }^{99}$ However, this kind of focus overestimated the relationship between social substratum and legal text, without considering the limits of the latter's normative force.

Concerning this matter, more than considering the particular conditions of the liberal ideas of codification in the context of the so-called "domestic privatism" and the choice for an individualistic liberalism that was dominant in $19^{\text {th }}$ century Europe, one should observe, firstly, the practical significance of the codification in the process of concretisation and applicationof civil law. How would an extremely liberal code in the matter of labour relations stand in a society that was still mostly rural, in which monoculture in the agrarian large properties was the main driving force of the economy and the social life? Between the rural landlords (associated with importing and exporting in the commercial sector) and all of those who depended on them or were socially excluded, a tiny urban middle class was still in development. In this context, whereas landowners could "be protected from attacks against their fundamental interests", the regime of "liberal franchises benefited only a measly number, being strange to the vast majority of the miserable and uncultured population". ${ }^{100}$ From the social-legal point of view, these were relations of over-inclusion and under-inclusion, i.e., between, respectively, those "above" and those "below" the civil law. After slavery was abolished, the vast majority of the population consisted of, in the words of Sílvio Romero and seconded by Orlando Gomes, the "impoverished by inertia", and not of rural and industrial workers who were capable of claiming their rights. ${ }^{101}$ With no access to elementary civil rights, these miserable people who roamed the countryside and towns and who could, at most, find jobs sporadically, engage in informal work relations or be incorporated as domestic servants, always outside the scope of the law.

With regard to the service contract, Pontes de Miranda - despite his racialist explanation of the problem ${ }^{\mathbf{1 0 2}}$ - was aware of the incompatibility

98 Pontes de Miranda (1981 [1928]) 443.

99 Gomes (1958) 66.

100 Gomes (1958) 46.

101 Romero (1894) XXXIV; Gomes (1958) 39-40.

102 See above note 88. 
between the Code's liberal individualism and "menial relations": "the Brazilian servants did not claim - it was as if they continued to serve, freed, the old lord; the attempts to modernise the service contract were unsuccessful, because they were hindered by a double indifference: of the master and the domestic servant". ${ }^{103}$ Without a doubt, a double indifference founded on the extreme asymmetry of positions, which made the former immune to obligations and the latter deprived of rights. This situation, despite all the transformations in labour law in Brazil, in part persists among the domestic service relations which are so abundant in the country.

One cannot entirely deny that the promulgation of a liberal individualist civil code in a context of extreme social inequality - with labour relations still permeated by slavery - may be interpreted as a "copy", "importation" or "transplant" of foreign law with "inevitable deformations" and no commitment to the country's social reality. ${ }^{104}$ It is from this perspective that one should attempt to understand the statement by Pontes de Miranda: "The Brazilian Civil Code, in what it concerns Clóvis Bevilaqua, is a codification for the Law Schools more than for life". ${ }^{105}$ Orlando Gomes, when referring to the transplant of "alien institutions, which in those regions [of more "advanced"peoples] have begun to wither", claimed that the Code "anticipated itself to reality", "presenting itself as an approximation of the reality of the future" 106 This ambivalence suggests that it was not simply a "transplant", "importation" or "copy" of institutional artefacts.

The private codification in the second decade of the $19^{\text {th }}$ century must be understood in the context of the abolition of slavery and of liberal constitutionalisation due to the proclamation of the Republic. Here, also, the liberal ideas of individualist private law were permeated by local conditions, from the perspective of both the structure of normative texts and the legal practice.Liberal legal semanticswas not only pervaded by anti-liberal or nonliberal notions of the jurists, but also by social structures that were incompatible with liberal individualism. This phenomenon impacted the very text of the code, particularly family law. Therefore, in the sphere of legal structures, textual institutionalisation implied a certain dislocation of the

103 Pontes de Miranda (1981 [1928]) 445-446.

104 Gomes (1958) 45.

105 Pontes de Miranda (1981 [1928]) 86-87.

106 Gomes (1958) 43 and 72.

72 Marcelo Neves 
liberal ideas on codification. Nonetheless, the greater issue seems to relate to the limits of the normative-legal concretisation of the Code in an adverse social context. How does liberal individualism present itself in relationships of dependency developed within networks that stabilise expectations of favour exchanging, reciprocal benefits, loyalties and urgent supplying of needs beyond the margin of codified civil law? In these conditions, its meaning and functions dislocated.

This does not mean, nevertheless, that civil codification based on individualist liberalism was socially irrelevant. The Code's weak normative-legal force was connected to its political-symbolic function which was ambivalent, such as the liberal Constitution's one. On the one hand, it constituted a selfillusion of codification as an expression of the emancipation of civil relations in Brazil, which would be approaching the codifying experience in Europe; on the other, the invocation of the Code served as a form of criticism of the legal and political practices developed beyond the scope of the code itself. In the terms of this ambivalence, a postponement of its complete fulfilment to an uncertain future impregnated the legal and political debate about the Code. One did not consider, however, that a satisfactory legal-normative concretisation of the Civil Code would presuppose radical transformations in the social structure in Brazil at the beginning of the $20^{\text {th }}$ century, an unlikely alternative in the historical horizon of possibilities for Brazil in the world society at that time.

Are these misplaced ideas? It seems to be more appropriate to say ideas in another place, among so many places in the world society. Nevertheless, given that liberal codification ideas were part of world society, could we not say that they are ideas in the same place? This paradox between global and local or regional nurtures the reproduction of political and legal ideas at least as long as states exist as territorially delimited organisations: migrant ideas in a unitas multiplex.

\section{$5 \quad$ Final Observations}

The issues addressed here concerning the function and meaning of the liberal constitutionalisation of 1891 and the codification of civil law in 1916, impregnated by liberal ideas established in Europe and the United States since the late $18^{\text {th }}$ century, were not an isolated phenomena in the development of political and legal ideas and institutions in Brazil. Similar variables 
regarding the migration of ideas and the transmutation of institutions persisted throughout the legal experience of the $20^{\text {th }}$ century, leading to problems that endure - evidently in very different ways and measures - until today.

The same problems were reposed in the presence of "critical" legal thought in the perspective of a social or even socialist state during the 1920s and 1930s: social-democratic ideas, although adopted by the Constitution of 1934, were of little significance to the majority of the population; and the authoritarian ideas of the Constitution of 1937, in addition to not being "authentically national", as its defenders claimed, ${ }^{\mathbf{1 0 7}}$ had their legal efficacy suspended during the entire stretch of the "New State" (1937-1945) due to a transitory disposition (article 187). ${ }^{\mathbf{1 0 8}}$ The search of this "critical" thought for a "real, profound country", as opposed to alien ideas, was not new and could not, in any way, be linked to the modern art week of $1922 .{ }^{109}$ The subject of a "real country" as opposed to an "official" or "legal country" has been discussed since the second half of the $19^{\text {th }}$ century. What changed was the content of the debate, which focused on the adequacy of social-democratic, socialist and fascist ideas for the Brazilian legal-political context. However, these ideas were not more nor less authentically Brazilian than the individualist liberalism that was previously dominant. In both cases, they were ideas in another place of the world society.

The migration or shifting of individualist liberal ideas oriented towards constitutionalisation and codification should not be interpreted, in a generic manner, in terms of symmetric relations in the world society. That is, saying that they were "in the air" ${ }^{\mathbf{1 1 0}}$ is not enough. Although dealing with this issue merely as a search for "causal influences" ${ }^{\mathbf{1 1 1}}$ is impertinent, one cannot deny

107 LOEWENSTEIN (1942) 122 characterised it as "international tutti-frutti" and "constitutional cocktail”.

108 However, article 180 of the Constitution of 1937 stated that it was up to the President of the Republic to "issue law-decrees on all matters of legislative competence of the Federal Government".

109 See above note 1.

110 As suggested by Lopes and Garcia Neto (2009) 2, in relation to "critical" thinking in Brazil in the 1920s and 19330s. In the definitiveversion published in Spanish, these authors removed this expression from the quoted text (see idem [2011] 106), but the argument remains in the article.

111 Lopes and Garcia Neto (2009) 2. 
asymmetries in the circulation of these (and other) legal-political ideas within world society when they move through various states. ${ }^{\mathbf{1 1 2}}$ Concerning the post-colonial Latin-American period, being unaware that there were centres that were predominantly irradiators and peripheries that were primarily receivers of liberal ideas meant leaving aside some crucial aspects of the legal and political experience of these countries. ${ }^{113}$ Regarding Brazil, the presence of a certain cultural colonialism is undisputable. ${ }^{\mathbf{1 1 4}}$ In this sense, one cannot completely exclude the terms "importation", "transplant", "copy" and "imitation", among others, as well as the notions of "deformation" of ideas and "impurity" of theories. ${ }^{\mathbf{1 1 5}}$

However, this does not mean that they are "misplaced ideas" because, permeated by local semantics and conditioned by structures of the world society in the respective state, they have significance and perform functions in terms of legal and political institutions and of social practices. In this respect, embodied at the institutional sphere of legal and constitutional textualising, liberal ideas, already pervaded by inescapable local political and legal demands, exerted primarilya symbolic function, to the detriment of their normative-legal force. And the symbolic function serves ambivalently both to deny and enforce or expand rights. In another perspective, Schwartz - in an essay revising his own original formulation about "misplaced ideas" affirmed, in reference to imperial Brazil, something that could come close to this formulation:

112 The conception that Eisenstadt's model of multiple modernity does not support asymmetries seems mistaken. In Eisenstadt's work (2000), when speaking of the "origin" of modernity in Western Europe and of the "Euro-Western modernity transformation in America (the USA example)", of the transformation of "western modernity in Asia (the Japan example)" and of the "fundamentalism as modern movement against modernity" (these are the titles of the chapters in Eisenstadt's book), an asymmetry is pointed between centres that irradiate structures and ideas (semantics) and social spaces that reproduce,transform or confront them.

113 "It is not only about relativizing the opposition between local and universal, but also of seeing the perverse reciprocities between former colonies and imperialist nations, underdeveloped and developed, peripheral and central, etc., political oppositions more revealing and meaningful" (Schwarz [2012] 170). On this subject, the criticisms levelled at Schwarz's position by Franco (1976) and Bosi (2010), especially 400, appear to tend towards simplification. See note 42, above. Cf. also Schwarz (2012) 171-172.

114 Montoro (1973).

115 Medina (2008). 
"Well, in the former colonies, based on forced labour, liberalism does not describe the real course of things - and in this sense it is a misplaced idea. However, this does not prevent it from having other functions. For instance, it allows the elites to speak the most advanced language at the time and to, simultaneously, take advantage of slave labour at home. Less hypocritically, it can be an ideal of equality before the law, for which the dependent and slaves fight. The range of its functions includes utopia, real political objective, the distinctiveness of class and pure cynicism, but excludes the believable description of everyday life, which renders it realistically dignified in Europe." 116

Nevertheless, it seems that the conception of "misplaced ideas" should be eliminated, as well as its corollary, the non-description of reality. Firstly, it is important to consider that the liberal ideas of a constitutional or legal nature were related to the normative dimension of social structures, not having a primarily descriptive function. Therefore, in the law field, the main issue is not to describe "reality". Secondly, distinguishing between "legal" or "official" and "real" is not appropriate within the legal scope of constitutionalisation and codification. The inefficiency of "legal" or the distance of the "official" in relation to the "people" or, rather, the majority of the population is part of the political-legal "reality", which implies everyday practices. Consequently, I insist that instead of "misplaced ideas", it would be more appropriate to say that liberal ideas undertook different functions in the various political-legal loci organised into states; however, they belong to the semantics of the world society in which they circulate. Thus, the conclusion: the liberal ideas incorporated into the Brazilian Constitution of 1891 and the Brazilian Civil Code of 1916 were, paradoxically, ideas in another place (the society in the scope of the Brazilian state) and in the same place (the world society).

116 Schwarz (2012) 170-171. See above note 5.

76 Marcelo Neves 


\section{Bibliography}

Alves, José Carlos Moreira (2003), A parte geral do projeto de Código Civil Brasileiro (subsídios históricos para o novo Código Civil Brasileiro), $2^{\text {nd }}$ ed., São Paulo: Saraiva

Barbosa, Rui (1902), Parecer do Senador Ruy Barbosa sobre a redação do projecto da Câmara dos Deputados, in: Diário do Congresso Nacional, suplemento ao Nr. 126 de 27 de julho de 1902, Rio de Janeiro: Imprensa Nacional

Barbosa, Rui (1904), Réplica do Senador Ruy Barbosa às defesas da redação do projecto da Câmara dos Deputados, Rio de Janeiro: Imprensa Nacional

BARbosa, Rui (1932-1934), Comentários à Constituição Federal Brasileira, edited by Homero Pires, São Paulo: Livraria Academica/Saraiva, 1932 (Vol. I), 1933 (vols. II e III), 1934 (Vol. V)

Barbosa, Rui (1968), Código Civil: Parecer. Obras completas de Rui Barbosa, Rio de Janeiro: Ministério da Educação e Cultura, Vol. XXXII (1905), Tome III

Barreto, Tobias (2000), A questão do poder moderador (O governo parlamentar no Brasil) [1871], in: Barreto, Tobias, Estudos de direito. Campinas: Bookseller, $375-424$

Bevilaqua, Clóvis (1906), Em defeza do projecto de código civil brazileiro, Rio de Janeiro, São Paulo, Belo Horizonte: Livraria Francisco Alves

Bevilaqua, Clóvis (1975), Características do direito pátrio (1922), in: Bevilaqua, Cuóvis, Obra filosófica, Vol. II: Filosofia social e jurídica, São Paulo: Editora da Universidade de São Paulo, Editora Grijalbo, 185-212

Bosi, Alfredo (1992), A escravidão entre dois liberalismos, in: Bosi, Alfredo, Dialética da colonização, São Paulo: Companhia das Letras, 194-245

Bosi, Alfredo (2010), Um nó ideológico - sobre o enlace de perspectivas em Machado de Assis, in: Bosi, Alfredo, Ideologia e contraideologia: temas e variações, São Paulo: Companhia das Letras, 398-421

Brandão, Wilson (1980), Antônio Coelho Rodrigues (Ensaio de bibliografia e crítica), in: Coelho Rodrigues, Antônio, 1980, 1-33

Brunkhorst, Hauke (1999), Heterarchie und Demokratie, in: Brunkhorst, Hauke, Peter Niesen (eds.), Das Recht der Republik, Frankfurt am Main: Suhrkamp, $373-385$

Buarque de Holanda, Sérgio (1985), Do Império à República. Vol. II.5 da História Geral da Civilização Brasileira, edited by Sérgio BuARque de Holanda, $4^{\text {th }}$ ed., São Paulo: DIFEL

Buarque de Holanda, SÉrgio (1988), Raízes do Brasil, $20^{\text {th }}$ ed., Rio de Janeiro: José Olympio [1 $1^{\text {st }}$ ed. 1936]

Bueno, José Antonio Pimenta (1857), Direito Publico Brazileiro e Analyse da Constituição do Império, Rio de Janeiro: Villeneuve

Bueno, Ruth (1972), Regime jurídico da mulher casada, $3^{\text {rd }}$ ed., Rio de Janeiro: Forense 
Cardoso, Fernando Henrique (1985), Dos governos militares a Prudente-Campos Sales, in: Boris Fausto (ed.), História geral da civilização brasileira, $4^{\text {th }} \mathrm{ed}$., tome III, Vol. 1, São Paulo: DIFEL, 13-50

Carone, Edgard (1969), A Primeira República (1889-1930): texto e contexto, São Paulo: DIFEL

Carvalho, José Murilo de (1996), A construção da ordem/Teatro de sombras, $2^{\text {nd }}$ ed., Part II: Teatro de sombras: a política imperial, Rio de Janeiro: Editora UFRJ, Relume Dumará

Coelho Rodrigues, Antônio (1980), Projeto do Código Civil Brasileiro, $2^{\text {nd }}$ ed., Brasília: Ministério da Justiça

Comissâo Especial da Câmara Dos Deputados (1902), Parecer da Comissão Especial, in: Projecto de Codigo Civil Brazileiro - Trabalhos da Comissão Especial da Câmara dos Deputados, Vol. VIII, Rio de Janeiro: Imprensa Nacional, 3-41

Constant, Benjamin (1872), Réflexions sur les constitutions et les garanties, avec une Esquisse de Constitution (1814-1818), in: Constant, Benjamin, Cours de politique constitutionnelle ou collection des ouvrages publiés sur le gouvernement representative, $2^{\text {nd }}$ ed., Vol. II, Paris: Librairie de Gillaumin, 167-217

Constant, Benjamin (1957), Principes de politique [1815], in: Constant, Benjamin, Oeuvres, edited by Alfred Roulin, Paris: Gallimard, 1063-1215

Dantas, San Tiago (1962a), Figuras do direito, Rio de Janeiro: José Olympio

Dantas, SAN Tiago (1962b), Rui Barbosa e a renovação da sociedade, in: Dantas (1962a) 21-39

Dantas, San Tiago (1962c), Rui Barbosa e o Código Civil, in: Dantas (1962a) 41-78

Dantas, San Tiago (1962d), Ciência e consciência - um estudo sobre Clóvis Bevilacqua, in: Dantas (1962a) 79-93

Eisenstadt, S. N. (2000), Die Vielfalt der Moderne, trad. alemã B. Schluchter, Frankfurt am Main: Velbrück

Faoro, Raymundo (1976), Machado de Assis: a pirâmide e o trapézio, $2^{\text {nd }}$ ed., São Paulo: Editora Nacional/Secretaria de Cultura, Ciência e Tecnologia do Estado de São Paulo

Faoro, Raymundo (1985), Os donos do poder: formação do patronato político brasileiro, Vol. 2, $6^{\text {th }}$ ed., Porto Alegre: Globo [ $1^{\text {st }}$ ed. 1958]

Fonseca, Ricardo Marcelo (2011), A modernização frustrada: a questão da codificação civil no Brasil do século XIX, in: Teixeira, Ana Carolina Brochado, Gustavo Pereira Leite Ribeiro (eds.), Manual de teoria geral de direito civil. Belo Horizonte: Del Rey, 13-34

Fonseca, Ricardo Marcelo (2012), A noção de imaginário jurídico e a história do direito, in: Fonseca, Ricardo M. (ed.), Nova história brasileira do direito: ferramentas e artesanias, Curitiba: Juruá, 19-29

Franco, Maria Sylvia de Carvalho (1976), As ideias estão no lugar, in: Cadernos de Debate 1, São Paulo: Brasiliense, 61-64

Freitas, Augusto Teixeira de (1878), Aditamento ao Codigo do Commercio, Vol. 1, Rio de Janeiro: Perseverança 
Freitas, Augusto Teixeira de (1983), Código Civil - Esboço, 2 vols., Brasília: Ministério da Justiça/Fundação Universidade de Brasília [ $1^{\text {st }}$ ed. Rio de Janeiro, 1864]

Freitas, Augusto Teixeira (2003), Consolidação das Leis Civis, 2 vols., Brasília: Senado Federal [ed. fac-similar da $3^{\text {rd }}$ ed. Rio de Janeiro: Garnier, 1876] [1 ${ }^{\text {st }}$ ed. 1857]

Gomes, Marcelo (2011), Trabalho doméstico - economia aquecida gera escassez de trabalho doméstico, in: Desafios do Desenvolvimento, Vol. 8, Nr. 66, Brasília: IPEA, 62-67

Gomes, Orlando (1958), Raízes históricas e sociológicas do Código Civil brasileiro, Salvador: Universidade da Bahia

Grinbeg, Keyla (2002), O fiador dos brasileiros: cidadania, escravidão e direito civil no tempo de Antonio Pereira Rebouças, Rio de Janeiro: Civilização Brasileira

Grinbeg, Keyla (2008), Código Civil e cidadania, $3^{\text {rd }}$ ed., Rio de Janeiro: Zahar

IBGE = Fundação do Instituto Brasileiro de Geografia e Estatística (1987), Pesquisa Nacional por Amostra de Domicílios - 1987, Vol. 11/1, Rio de Janeiro: IBGE

Levaggi, Aberlardo (1988), Influencia de Teixeira de Freitas sobre el projecto de Código Civil Argentino en materia de relaciones de familia. Las fuentes utilizadas por Vélez Sarsfield, in: Shipani, Sandro (ed.), Augusto Teixeira de Freitas e il diritto latinoamericano, Pádua: CEDAM, 399-426

Loewenstein, Karl (1942), Brazil under Vargas, Nova York: Macmillan

Loewenstein, Karl (1975), Verfassungslehre, German transl. by Rüdiger Boerner, $3^{\text {rd }}$ ed., Tübingen: Mohr

Lopes, José Reinaldo de Lima, Paulo Macedo Garcia Neto (2009), Critical Legal Thought. Artigos Direito GV - Working Papers 37. São Paulo: Escola de Direito de São Paulo da Fundação Getulio Vargas, 33 páginas [Versão definitiva em espanhol (2011): Pensamiento jurídico crítico en Brasil (1920-1940), in: García Villegas, Mauricio, María Paula Saffon (eds.), Crítica jurídica comparada, Bogotá: Universidad Nacional de Colombia, 105-142]

Luhmann, Niklas (1975), Die Weltgesellschaft, in: Luhmann, N., Soziologische Aufklärung 2: Aufsätze zur Theorie der Gesellschaft, Opladen: Westdeutscher Verlag, 51-71

Luhmann, Niklas (1980), Gesellschaftliche Struktur und semantische Tradition, in: Luhmann, Niklas, Gesellschaftsstruktur und Semantik: Studien zur Wissenssoziologie der modernen Gesellschaft, Frankfurt sobre o Meno: Suhrkamp, Vol. 1, 9-71

Luhmann, Niklas (1997), Die Gesellschaft der Gesellschaft, Frankfurt sobre o Meno: Suhrkamp, 2 tomes [trad. esp.: La sociedad de la sociedad, México: Herder/ Universidad Iberoamericana 2007]

Machado De Assis, [Joaquim Maria] (1955), Crônica de 29 de dezembro de 1861 no Diário do Rio de Janeiro, in: Obras Completas de Machado de Assis, Tome 22: Crônicas, Vol. 1 (1859-1863), Rio de Janeiro, São Paulo, Porto Alegre: W. M. Jackson Inc. Editores, 1955, 102-109 
Machado Neto, A. L. (1969), História da idéias jurídicas no Brasil, São Paulo: Editora Grijalbo, Editora da Universidade de São Paulo

Mártinez Paz, Enrique (1927), Freitas y su influencia sobre el Código Civil Argentino, Córdoba: Imprensa de la Universidad

Maximiliano, Carlos (1948), Comentários à Constituição Brasileira, Vol. I., $4^{\text {th }}$ ed., Rio de Janeiro, São Paulo: Freitas Bastos

Medina, Diego Eduardo López (2008), Teoría impura del derecho. La transformación de la cultura jurídica latinoamericana, Bogotá: Legis

Meira, Sítvio (1983), Teixeira de Freitas: o jurisconsulto do Império, $2^{\text {nd }}$ ed., Brasília: Cegraf

Mercadante, Paulo (1980), A dualidade do direito privado, in: Mercadante, Paulo, A consciência conservadora no Brasil: Contribuição ao estudo da formação brasileira, $3^{\text {rd }}$ ed., Rio de Janeiro: Nova Fronteira, 177-194

Montoro, André Franco (1973), Filosofia do direito e colonialismo cultural: transplante de institutos jurídicos inadequados à realidade brasileira, in: Revista de Informação Legislativa, Vol. X, Nr. 37. Brasília: Senado Federal, 3-20

Moraes Filho, Evaristo (1982), Proteção do trabalhador agrícola, in: Moraes Filho, Evaristo, Direito do trabalho: páginas de história e outros ensaios, São Paulo: LTr, 99-115

Nabuco, Joaquim (1997), Um estadista do Império, $5^{\text {th }}$ ed., 2 vols., Rio de Janeiro: Topbooks [ $1^{\text {st }}$ ed., 3 vols., 1897-1899]

Neves, Marcelo (1992), Verfassung und Positivität des Rechts in der peripheren Moderne: Eine theoretische Betrachtung und eine Interpretation des Falls Brasilien, Berlin: Duncker \& Humblot

Neves, Marcelo (2005), Between Under-Integration and Over-Integration: Not taking Citizenship Rights Seriously, in: Souza, Jessé, Valter Sinder (eds.), Imagining Brazil, Lanham, Md: Lexington Books, 61-90

Neves, Marcelo (2006), Einige Probleme mit Niklas Luhmanns Auffassung von den Staaten der Weltgesellchaft, in: Soziale Systeme: Zeitschrift für Soziologische Theorie, Vol. 12, Nr. 2, Stuttgart: Lucius \& Lucius, 247-273

Neves, Marcelo (2008), Entre Têmis e Leviatã: uma relação difícil - o Estado democrático de direito a partir e além de Luhmann e Habermas, $2^{\text {nd }}$ ed., São Paulo: Martins Fontes

Neves, Marcelo (2009), Transconstitucionalismo, São Paulo: WMF Martins Fontes

Pacheco, Cláudio (1958), Tratado das Constituições Brasileiras, Vol. I, Rio de Janeiro, São Paulo: Freitas Bastos

Pontes de Miranda, Francisco Cavalcanti (1981), Fontes e evolução do direito civil brasileiro, $2^{\text {nd }}$ ed., Rio de Janeiro: Forense [ $1^{\text {st }}$ ed. 1928]

Ribeiro, Ernesto Carneiro (1902), Ligeiras observações sobre as emendas do Dr. Ruy Barbosa feitas à redação do Projecto de Código Civil, Bahia: Dois Mundos

Ribeiro, Ernesto Carneiro (1905), A redacção do Projecto do Código Civil e a réplica do Dr. Ruy Barbosa, Bahia: Dois Mundos

Rodrigues, José Carlos (1863), Constituição Política do Império do Brazil [...]. Rio de Janeiro: Laemmert 
Romero, Sílvio (1894), Doutrina contra doutrina: o evolucionismo e o positivismo na República do Brasil, $1^{\text {st }}$ series, Rio de Janeiro: J. B. Nunes

Romero, Ś́tvio (1960), História da literatura brasileira, Vol. 1, $6^{\text {th }}$ ed., Rio de Janeiro: José Olympio [ $1^{\text {st }}$ ed. 1888$]$

Roure, Agenor (1918-1920), A Constituinte Republicana, Rio de Janeiro: Imprensa Nacional, 1918 (Vol. II) - 1920 (Vol. I)

Santiago, Silviano (2002), Introdução, in: Santiago, Silviano, Intérpretes do Brasil, Vol. 1, Rio de Janeiro: Nova Aguilar, XIII-XLVIII

Schwarz, Roberto (2000), As idéias fora do lugar, in: Schwarz, Roberto, Ao vencedor as batatas: forma literária e processo social nos inícios do romance brasileiro, $4^{\text {th }}$ ed., São Paulo: Duas Cidades, Editora 34, 9-31 [1 ${ }^{\text {st }}$ ed. 1977] [Engl. transl.: Misplaced Ideas: Literature and Society in Late-NineteenthCentury Brazil, in: Schwarz, Roberto, Misplaced Ideas: Essays on Brazilian Culture, London/New York: Verso, 1992, 19-32]

Schwarz, Roberto (2012), Por que "ideias fora do lugar"?, in: Schwarz, Roberto, Martinha versus Lucrécia: ensaios e entrevistas, São Paulo: Companhia das Letras, 165-172

Sousa, Johquim Rodrigues de (1867), Analyse e Commentário da Constituição Política do Império do Brazil ou Theoria e Pratica do Governo Constitucional Brazileiro, Vol. I, São Luiz: B. de Mattos

Surgik, Aloísıo (1988), O pensamento codificador de Augusto Teixeira de Freitas em face da escravidão no Brasil, in: Shipani, SAndro (ed.), Augusto Teixeira de Freitas e il diritto latinoamericano, Pádua: CEDAM, 427-447

Torres, Alberto (1978), A organização nacional: Primeira parte, A Constituição, $3^{\text {rd }}$ ed., São Paulo: Editora Nacional [ $1^{\text {st }}$ ed. 1914]

Veronese, Alexandre (2013), Entre os dispositivos conceituais e a gramática: o direito e a política na formação do Código Civil de 1916, in: Revista da Fundação Casa de Rui Barbosa, Vol. 6, Rio de Janeiro: Casa de Rui Barbosa (forthcoming)

Vianna, Oliveira (1939), O Idealismo da Constituição, $2^{\text {nd }}$ ed., São Paulo, Rio de Janeiro, Recife, Porto Alegre: Editora Nacional

Villas Bôas Filho, Orlando (2009), Teoria dos sistemas e direito brasileiro, São Paulo: Saraiva

Wallerstein, Immanuel (2006), World-Systems Analysis: An Introduction, $4^{\text {th }}$ ed., Durham, London: Duke University Press 



\section{La denominada «crisis del derecho» desde la perspectiva argentina durante el periodo de entre guerras mundiales (1920-1940)}

\section{Introducción}

El claroscuro agonal de la época: del mundo cultural al ámbito jurídico

Si luego de la encrucijada de la primera guerra mundial, la literatura europea y americana vivió una etapa de pesimismo y de intensa reflexión en torno al valor de ciertos conceptos, fue evidente que también repercutió en el ámbito jurídico. La primera estuvo marcada por el resabio del pensamiento de Nietszche, al que se agregó el de Spengler. Fueron referentes Hesse, Proust, Joyce, Pirandello, y en la Argentina quienes ofrecían algunas notas pesimistas o que se abrían a ciertas novedades, en autores tales como Leopoldo Lugones, Roberto Arlt, Raúl González Tuñón, Jorge Luis Borges, entre otros. Ello produjo una intensa circulación de ideas, algunas de las cuales prosperaron en doctrinas y otras quedaron en el plano de la utopía. No extraña, entonces, que el horizonte de las principales obras jurídicas del período esté encuadrado en lo que llamaron la "crisis del derecho", dando por sentado que el derecho que había regulado la vida hasta entonces no sólo había sido ineficaz para evitar la conflagración, sino que resultaba incapaz para atender las novedades de la segunda revolución industrial, y menos aún resultaría apta para regir la problemática de la época que sobrevenía.

Esa dicotomía entre el mundo del «ayer» y el de la realidad de la posguerra, que tan magistralmente nos esbozó Paolo Grossi en su «Novecento giuridico», 1 siguiendo los pasos de la lacerante pintura que legó un eximio

1 Grossi (2011a); postura diversa a la de Mario Losano, que señala la brevedad de ese siglo, pues en su opinión, debemos partir del fin de la segunda guerra mundial. Grossi (1998), Losano (2011 y 1997). 
escritor como Stefan Zweig, señalando como aquel mundo donde las reglas que ordenaban la sociedad, la economía y el derecho se habían trastocado, bien pudo expresar que ese mundo del «ayer» donde todo estaba enmarcado, todo caía bajo determinadas reglas, cada uno sabía cuáles eran sus derechos y sus obligaciones, se había derrumbado. Exhibió así como la conflagración que se transformó en una insoslayable bisagra, al provocar un giro copernicano en las costumbres y una veloz circulación de ideas, trajo como consecuencia la misma desolación que la que mostraba un campo de batalla atravesado por la sangre y el dolor de los combatientes.

Justamente, dice Grossi, quien era la expresión de la fijeza de lo inconmovible, el emperador Guillermo II, dejaba atrás su mundo y se marchaba y, con él arrastraba el mundo de las certezas, y hacía desembocar a la sociedad en un sino que abarcaba la política, el mundo cultural, el económico y asimismo el jurídico. Para Zweig, mortal al fin, el mundo que él había conocido y vivido ya no existía, la realidad le devolvía una imagen irreconocible y dolorosa, y no había forma de retornar al pasado. El suicidio fue la única vía que halló para responder a esa situación. Pero, los pueblos no se suicidan, al menos en teoría, y de allí los estudios que se encararon en torno a la nueva realidad. Por eso, tal como advertimos, no extraña que el horizonte de las principales obras jurídicas estuviese encuadrado en ese concepto de crisis, pero no exenta de una toma de conciencia para asomarse al nuevo panorama que ofrecía cierta incertidumbre pero a la vez ciertas expectativas, y efectuar propuestas encaminadas a consagrar un concepto del derecho apoyado sobre nuevas bases. Cabe apuntar, que no todos coincidieron en éstas, pero la resistencia fue sobrepasada por la inexorabilidad de una realidad desbordante, que hizo trizas viejas y conocidas nociones.

La filosofía, y en particular la filosofía del derecho, a través de sus expertos, se encargaron de analizar el cuadro a través de obras que usaron el apotegma de la crisis; la enorme cantidad de éstas hace imposible su detalle o análisis singular en este artículo, y por ello nos remitamos a la nota, por razones de brevedad. $^{2}$ Es digno de destacar que el primero que llamó a la época moderna como «l'âge de l'inquiétude», fue Michelet, ${ }^{3}$ aunque se refería al siglo

2 En particular: Ortega Y Gasset (1932); Hespanha (2002) 22-30. Lopez De Oñate (1943) ver nota 9 de este libro, en la misma hay cita de numerosas obras, lo que demuestra la importancia del tema en la época en estudio; Butler (1933); Calamandrei (1939); Ruggiero (1927); Tilgher (1932); Lopez De OÑate (1939).

3 Hauriou (1923) 30. 
anterior, pero no hay duda que toda época se cree o se ve como una época de transición, ${ }^{4}$ porque pone el acento en sí misma.

Participo así del concepto que expuso Flavio López de Oñate, en su obra «La certezza del diritto», «la historia se presenta como «crisis continua), por medio de la cual, del presente y por encima del presente, nace trabajosamente el futuro». ${ }^{5}$ Respecto a la filosofía del derecho, advirtió que ella implicaba un acto de conciencia con la cual el hombre trataba de conocer en profundidad la vida del derecho. Se ocupaba de rescatar, en el contexto de su época, dos elementos de honda significación: la vivacidad y la realidad del derecho, que sorteaban el formalismo que había guiado la concepción jurídica positivista y el reconocimiento de la innegable interrelación del mundo cultural, - incluyendo en particular la literatura, que utilizó en varias oportunidades -, con el mundo jurídico. Lo comprobamos v.g. en su cita de Chesterton, para referirse a la relación entre libertad y certeza, señalando que «no se es libre mientras una institución no venga a liberarnos»; ${ }^{6}$ la certeza del derecho no podía separarse de la certeza de la ley, aunque ésta perdiese entidad en el período.

La crisis, en opinión del jurista que venimos analizando, servía, al adentrarse en las raíces de la realidad, para comprender la esencia profunda del derecho. Ello le hizo concluir a Giuseppe Capograssi, quién se ocupó de una suerte de prefacio en la publicación de la obra de ese joven jurista fallecido, que éste quedaría

«como una de las más patéticas y seguras interpretaciones de la crisis, y si la crisis, ... debiera continuar y seguir hasta el fondo, quedará como un grito, uno de los últimos gritos ... un himno, a la libertad, al derecho, al individuo, que hayan resonado en este Occidente que está en peligro mortal de hundirse poco a poco en la noche de la masa y de la horda. Si esa noche llega a extenderse sobre la historia, este librito quedará para el historiador del futuro como una de las más claras voces de alarma que hayan resonado en nuestro tiempo». ${ }^{7}$

4 Lopez De Oñate (1939) 59, remite a Leopardo, Dialogo di Tristano e un amico. Le prose morali, al cuidado de Della Giovanna, Firenze (1895) 272.

5 Lopez De Oñate (1939) 51.

6 Chesterton (1933), citado en Lopez De Oñate (1943) 87. Lopez De Oñate (1943) nota $5,53$.

7 Lopez De Oñate (1943), Ricordo di un amico de Giuseppe Capograssi, 192. Éste señaló que la repercusión de la obra podía observarse, entre otros autores, en CalamandreI (1943); Carnelutti (1943). Véase el resto de autores citados en la nota 1 de ese Recuerdo. 
En última instancia, implicaba y aún constituye, la conexión entre la condición humana y el derecho.

A su vez, el mismo Giuseppe Capograssi fue un defensor de la experiencia y del valor de la misma para la creación y la comprensión del derecho, pues más que el conocimiento puso el acento en la acción, y le reconoció a la ciencia jurídica un lugar de privilegio, ya que, por su historicidad, era la que ofrecía la mayor posibilidad de conocer el mundo humano, al dedicarse al descubrimiento de los valores más hondos del hombre singular, y agregaba que las demás ciencias tenían por objeto el reconocimiento de la vida, pero no se presentaban como maestras de ella, y subrayó que esa ciencia trabajaba en la historia en medio de la impureza de los intereses, de las pasiones, de las competiciones, pero eso no la disminuía sino aumentaba su valor. ${ }^{\mathbf{8}}$

Podríamos recurrir a muchos otros autores para mostrar la relación que había en esa etapa entre la crisis del derecho y la búsqueda de la certeza, como elemento fundamental del orden jurídico, y el peligro que se sentía frente a la incertidumbre derivada del trastrocamiento de ese mundo contemporáneo en todas sus expresiones, fuesen económicas, sociales, políticas, artísticas, etc. Sin embargo, a los fines del presente, estimamos que es suficiente con la muestra ofrecida.

Un nuevo escenario se desenvolvía frente a los ojos atónitos de quienes añoraban su pasado.

Palabras y conceptos claves en la Argentina: crisis, modernización, espíritu nuevo, novedades, realidad social, Estado. Superación de la incertidumbre de acuerdo a nuevos postulados

Crisis v. modernización

Si atendemos a esa nueva realidad que marcamos en la introducción, nos interesar encarar el análisis del cómo se resolvió la situación en la Argentina, ya no partiendo de la búsqueda de esa íntima conexión entre la condición humana y el derecho, de manera expresa - aunque siempre rondó en el paisaje ideológico un áurea nívea - sino quizás de manera menos etérea y más concreta, a través de la reforma jurídica. Y lo primero que se presenta ante nuestra vista es que, de una concepción amplia, absoluta, de los dere-

8 CAPograssi (1943) VIII; CAPOGRASSI (1932).

86 Maria Rosa Pugliese 
chos individuales y en especial del contrato, se pasó a otra restringida. Si bien las novedades produjeron desazón en muchos, otros visualizaron o entablaron la indagación de soluciones con un espíritu nuevo. Es decir, que ante la caída inexorable del mundo del «ayer», la incertidumbre se trató de encarar con otro espíritu. Así, al margen de las contingencias sociales y económicas que llevaron en un principio a un desenfreno, ante la caída de las pautas morales otrora establecidas, luego asomó la necesidad de limitar ciertos derechos, o quizás darles otro encuadre, pues un ejercicio extensivo de aquéllos producía muchas veces la opresión de una parte de la sociedad por mano de otra. De allí, asimismo, una visión diversa del principio de igualdad que tanto se preconizaba y poco se cumplía, y del derecho de propiedad, que de pronto se encontraban enfrentados con el espejo de la realidad. Ello quedó reflejado en la denominada «cuestión social», que llevó a una reelaboración de la relación de empleo y su exclusión paulatina del ámbito iusprivatístico para ingresar decisivamente en la esfera iuspublicista.

Observamos, entonces, que hubo palabras y conceptos claves que aparecieron o que adoptaron otras características en la época. Algunos autores se adelantaron en el tiempo para introducirlos o referirse a los mismos, - el caso de Carlos Octavio Bunge - ${ }^{9}$ pero ahora cobraban un dinamismo generalizado. En particular, podemos hacer referencia a «crisis», «modernización», «espíritu nuevo», «novedades», «realidad social». A su vez, se modificaron estructuralmente los conceptos de «autonomía de la voluntad» y «contrato», y se hizo referencia a nuevos principios e institutos para atender las «novedades». En este caso, se trató de superar la incertidumbre que emanaba de la nueva realidad social, a través de un análisis diverso de la naturaleza de aquéllos.

Una advertencia previa fundamental. La voz crisis, ${ }^{\mathbf{1 0}}$ por su generalidad, no puede considerarse exclusiva de una determinada época, sino que implica un revulsivo que agita las aguas de una ciencia, en pos de un cambio más o

9 Bunge (1916); Bunge (1907).

10 Huizinga (1933), (1938), (1934); Guenon (1927); Belloc (1937); Butler (1933); KeyserLing (1935); Kuntz (1926); Strowski (1931). Flavio Lopez de Oñate señalaba que las principales obras dedicadas al tema de la crisis eran: Berdiaeff (1931); Scheler (1936); Valery (1931); Ortega Y Gasset (1932); Spengler (1919). El aspecto técnico se observa en Veit (1937) y Dessauer (1927); Perticone (1942); De Stefani (1941); Robbins (1935); Delaisi (1925); Pareyson (1940) con abundante bibliografía; Hauriou (1923); Cattaneo (1892); SOREL (1941). 
menos drástico de sus postulados. Sin embargo, en el momento que estudiamos, nos exhibe el protagonismo de sus cultores y su convencimiento de que se había dado una vuelta sin retorno de los valores que habían constituido el sostén del último tercio decimonónico y la irrupción de nuevos paradigmas jurídico-políticos, conectados con una visión desacralizada de la sociedad, el poder y el derecho, emergiendo tras un proceso de secularización, que, ejecutado con fuerza en el siglo XIX, se proyectó decisivamente en las siguientes décadas del siglo XX.

¿Qué implicaba para esos autores la voz «crisis»?

Ella provenía del ámbito cultural antes de ingresar en el jurídico, tal como señalaba Flavio López de Oñate, ${ }^{\mathbf{1 1}}$ quien afirmó que la crisis de su época, o sea la previa a la segunda guerra, era una crisis del individuo, que anhelaba la certeza, pero no la podía conseguir, por efecto de la situación política-económica-social que se vivía. Partía para eso del concepto de angustia, que expresaron Heidegger y Jaspers, Berdiaeff y Scestov, Wahl y Marcel, Barth y Tillich, Wust y Guardini, y antes, Unamuno. Había un problema de certeza, es decir, hubo una búsqueda anhelosa de ella, y eso provenía del individuo.

Al respecto, podemos interrogarnos: ¿hay crisis del derecho o de sus fuentes? Puede haber crisis del Estado, crisis del órgano generador de la ley, y a su turno, ¿qué pasa con quienes deben interpretarla o aplicarla? ${ }^{\mathbf{1 2}}$

Para Giner de los Ríos, el derecho se manifestaba en una serie de estados particulares, mudables y sucesivos, pero no perdía su inagotable fondo, que era lo que constituía la vida del derecho. ${ }^{13}$

Si seguimos el pensamiento de Giorgio Del Vecchio, y en particular a través del comentario de un juez argentino de la época, Alberto M. Justo, «el derecho y su ciencia están en perpetua crisis». Ello, basado en el maestro italiano, pues «hay un continuo esfuerzo y trabajo de adaptación y de reintegración». ${ }^{14}$

11 Lopez De Oñate (1943) 55 y ss.

12 Grossi (2006); Lopez De Oñate (1943) cita a Carnelutti (1930); Carnelutti (1937) 167 y ss.; Morin (1927); Ruggiero (1927); Mossa (1930); Calamandrei, prefacio a la Jemolo (1932); Battaglia (1929); Giannini (1939), en ésta hay cita de literatura alemana; BONNARD (1939).

13 Giner De Los Rios (1916) 64.

14 Del Vecchio (1935), analizado por Alberto M. Justo (1938) 41; Del Vecchio (1933); Del Vecchio (1936); Del Vecchio (1938). 
A su vez, no puede desconocerse que la conciencia social buscaba otros canales de expresión, que no eran precisamente los del derecho tradicional. ${ }^{\mathbf{1 5}}$

Aunque en nuestro concepto debe hablarse de crisis de las fuentes, llama la atención y no puede soslayarse que, entre 1920 y 1940, una serie de obras, tal como adelantamos, se refiriesen a la crisis del derecho y utilizasen ese término en el título de las mismas. Así como apuntaron a la «modernización del derecho», caracterizando esta etapa. Modernizar era dejar atrás los principios que habían regulado la vida bajo inspiración individualista, para entrar decididamente en la alta consideración del mundo que surgió después de la primera guerra. Y en éste, lo social empujaba, desbordaba, daba nuevo sentido al derecho.

\section{Crisis v. realidad social}

Señalamos que la época estuvo signada por una nueva realidad social, ésta vino acompañada por el protagonismo de otros actores, antes casi invisibles a la sociedad y a la economía y que se representaron bajo el denominador: «cuestión social», producto de la extensión y vulgarización de inventos y de nuevos medios que ofrecía la ciencia, el desarrollo del transporte y de la economía, que volcó al mercado una cantidad enorme de personas que provenían del ámbito rural o que eran el resultado del crecimiento demográfico por vía de la inmigración en las zonas urbanas de la Argentina. Aunque una gran parte se ubicó en la ciudad de Buenos Aires, otros se instalaron en el interior, preferentemente en ámbitos urbanos, sin desdeñar los rurales. Ello motivó, entre otros, problemas de viviendas que se trataron de paliar a través de leyes de emergencia de alquileres y desalojos. Aquella otrora creencia del iluminado progreso indefinido se dio de bruces con la oscuridad de los añicos de la Bolsa y de una moneda sin respaldo. Los quebrantos comerciales y la desocupación, fueron como repiques de campana que no podían desoírse.

El desafío, que no era solo argentino, requería nuevos modelos y aparecieron renovados paradigmas. La realidad empujaba la crisis. Sin duda, la realidad fue la proa de los cambios, y la presunta incertidumbre que provocaron en variados campos, constituyó a su vez, la certeza que requerían otros. 
Crisis v. certeza

Existió, así, una íntima y extrema necesidad de superar la incertidumbre y alcanzar la certeza jurídica. ${ }^{16}$ La crisis justamente tenía como argumento la incertidumbre que derivaba de las situaciones vividas luego de la guerra y la persecución anhelante de la seguridad. Pero si para algunos la ley no daba seguridad o certeza, la constante renovación de esa fuente aparejaba a la vez, paradojalmente, una cierta incertidumbre, por eso Carnelutti decía que era «mejor soportar una ley mala que cambiarla demasiado a menudo» y agregaba «la ley no solo se hace para mandar sino también para que dure. No puede ser naturalmente, eterna; pero debe ser longeva. Toda mutación ... representa una turbación de equilibrio ...», y así arguía sobre la inquietud legislativa. ${ }^{\mathbf{1 7}}$

Esto nos convence que la época ofrecía un muestrario disímil de opiniones para enfrentar sus dilemas. Si bien hubo coincidencia en la crisis de las fuentes, la solución no estaba únicamente en la reforma legislativa, aunque en la Argentina, ese haya sido el camino.

La plenitud del orden jurídico como elemento para la certeza

Se discutió técnicamente sobre la plenitud del orden jurídico, como lo hicieron Norberto Bobbio ${ }^{\mathbf{1 8}}$ en Europa, y Carlos Cossio, ${ }^{19}$ en la Argentina. Ambos, y otros, se introdujeron en un intrincado terreno como es el de los principios generales del derecho y su interpretación, para alcanzar, según flotaba en el ambiente, un «derecho más justo», ${ }^{\mathbf{2 0}}$ y de esta manera cambió el papel tradicional de las fuentes jurídicas. ${ }^{21}$ Se llegó a las mismas costas del poder judicial, para desbordarlas, iniciando el proceso que de la interpretación pasó a la creación jurisprudencial propiamente dicha, aunque los jueces fueron los primeros en negar terminantemente que ellos fueran más allá del ordenamiento, sino que a lo sumo ajustaban la norma a la

16 Lopez De Oñate (1943); Pugliese (2010).

17 Carnelutti (1930) 180; Carnelutti (1940).

18 Bobbio (1993); Bobbio (1941) 4; Romano (1918).

19 Cossio (1939); Cossio (1941).

20 Justo (1937) 41. Anastasi, en varias notas de Jurisprudencia Argentina y La Ley, en particular Prólogo al I Repertorio de La Ley (AnAstasi [1938a]).

21 Diaz Couselo (1986).

$90 \quad$ Maria Rosa Pugliese 
realidad que debían resolver. ${ }^{22}$ Claro que, lo que implicaba certeza para algunos, traía aparejado el sentido contrario para otros.

En la Argentina, autores como Juan A. Bibiloni y Alfredo Colmo abrevaron sobre todo en fuentes europeas, pero también hubo otros que se abrieron a la problemática latinoamericana, sin perder de vista la europea, y aquí podemos citar a Leónidas Anastasi, Alfredo Palacios, Tomás Jofré, entre varios y agregar a Eduardo Couture, en Uruguay.

\section{Crisis v. realidad social}

La crisis, como expresamos, surgió de la realidad, y ésta implicó un desafío que había que domar. Si el derecho debía atender la nueva situación socioeconómica, debía cambiar el concepto con el cual se había consustanciado hasta entonces. Si el siglo se había vuelto atormentado y renovador, el rescate debía provenir de lo social y de la cultura que lo comprendía. El derecho debía ampararlo, y probablemente, el mejor mecanismo para lograrlo se encontró en la actividad judicial. Ella, pues, era quién estaba en mejores condiciones de atenderlos, estaba en contacto cotidiano con ellos. De allí la importancia que adquirió y sobre la cual volveremos.

Esto, a su turno, influyó en el tema de los valores, pues el individualismo dejó paso a un criterio más solidarista y hallamos muchas figuras que hablaron de colectivismo; y la secularización que ya mentamos, pese a embates de raíz cristiana, llegó para quedarse.

No podemos dejar de señalar la postura extrema de un jurista como León Duguit, visitante de la Argentina a comienzos de siglo XX, quién brindó conferencias que ofrecieron un refluir de ideas y participación de intelectuales. En su opinión, nos enfrentábamos a la sustitución de un sistema de orden metafísico e individualista, por un sistema jurídico de orden realista y socialista. Esas ideas, aunque no hayan calado hondo, dejaron una impronta, que podía aflorar en condiciones especiales. Decía que, «el derecho es mucho menos la obra del legislador que el producto constante y espontáneo de los hechos», y agregaba, «el texto queda vacío, sin vida, o mediante una exégesis sutil, se le da un sentido y un alcance que no soñó siquiera el legislador al redactar». ${ }^{23}$

22 Pugliese (2007); Pugliese (1994); Tau Anzoátegui (2011); Pugliese (2000); Abasolo, Leiva, Pugliese (2001).

23 Duguit (1905); Duguit (1901), entre otras obras. 
La realidad que esbozamos confluyó, asimismo, en un nuevo rol del juez, que se animó con mayor ímpetu a traducir los nuevos criterios de interpretación que nacían del derecho. Nunca admitieron crear, pero es evidente que lo hicieron y a menudo. Ello quedó reflejado en la tarea de los juristas abocados a la redacción del anteproyecto de Código Civil, luego de su designación en 1926. Las innovaciones fueron recogidas por este cuerpo y los miembros de la Comisión lo expresaron sin retaceos. Por lo tanto, es obvio que el estrecho marco de las fuentes del derecho, se vio agrandado por esta tarea silenciosa y cotidiana de los magistrados de primera y segunda instancia, y también por los del máximo Tribunal. Eso nos enfrenta con la consideración de si pudo llegarse a una cierta discrecionalidad del juez, que ocupaba un espacio entre el estrecho margen de un «étre inanimé», como pretendía Montesquieu, en su Esprit des lois, y menos en la «subsumtionsmaschine», según surgía de la literatura alemana. ${ }^{24}$

\section{Crisis v. Estado}

En cuanto al Estado, fue notorio que paulatinamente se pasó del modelo liberal encarnado en la doctrina del laissez faire, vigente en el pensamiento y la acción de los liberales positivistas de la generación del '80, que se continuó con matiz spenceriano en el Juez y Presidente de la Corte Suprema, Antonio Bermejo; al surgimiento de un nuevo modelo de organización, sobre cuya identidad y conformación, se tejen variadas versiones. Si algunos autores han apuntado a una desintegración del Estado por la aparición de otras múltiples organizaciones que exigieron un espacio, por otra parte, tampoco puede soslayarse que en especial en la década del '20, y pese a las mencionadas irrupciones, el Estado adquirió un neto corte intervencionista, que se hizo notorio en la siguiente, con ciertos matices particulares al final de la mis$\mathrm{ma}^{25}$

Aquí resulta imperioso tratar de encontrar algún eslabón que explique esta aparente contradicción y la supere. Que el Estado haya dejado su papel de «gendarme» de los derechos individuales, no implicó, a su turno, que haya

24 Lopez De Oñate (1943) 117 se ocupó de efectuar una relación sumamente interesante, y además de Montesquieu, citó a Binder; Ferrara (1911).

25 Zimmermann (1995); Pugliese, La Corte Suprema de Justicia del intervencionismo estatal, 1929-1947, en Historia de la Corte Suprema de Justicia, dirigido por Alfonso Santiago (h). 
desaparecido fagocitado por multitud de asociaciones, gremios, sindicatos, etc. Esta avalancha no fue sino la irrupción de la realidad social en el ámbito político, económico y jurídico. Ya no sirvieron los cartabones decimonónicos, ni los atisbos del comienzo de siglo para dar basamento al Estado. La realidad conformó y modeló una nueva figura de Estado. Y éste se resistió a perder terreno, y se corporizó de otra forma.

En el primer caso se privilegiaron intereses de tipo social, pudiendo señalar que aunque no se utilizó la figura de los intereses colectivos o difusos que se proyectó mucho más tarde, se desarrolló la idea de proteger algunos intereses que emanaban de una acuciante realidad, a favor de grupos que exhibían necesidades que no podían resolverse con criterios que aparecían como perimidos, o que de aplicarse en forma literal, producirían efectos nocivos para la sociedad. De allí que la afectación de derechos individuales derivados por ejemplo del derecho de propiedad, no se consideraron ilegítimos, pues de lo contrario se verían lesionados otros valores de interés general y por ende superiores, que afectaban a un mayor número de habitantes o ciudadanos. ${ }^{26}$ Se desenvolvió la doctrina por la cual la actividad de la Administración Pública, tenía por objeto la satisfacción de necesidades públicas y la protección de intereses colectivos. ${ }^{27}$

En el segundo, el derecho se recluyó exclusivamente en el ámbito positivo, es decir, que, aunque se hacía mérito de la moral y las buenas costumbres, éstas, más allá que en algunos cultores era expresión de matices trascendentes, emanaban de la propia vida social. Como reseñamos, una visión desacralizada de la sociedad, el poder y el derecho, emergía del movimiento secularizante del siglo XIX, pero ahora con un carácter diverso, con la consiguiente formulación de nuevos paradigmas jurídico-políticos. ${ }^{28}$ De ahí el pasaje del modelo liberal de Estado al intervencionismo de éste. Lo social era lo detonante.

Por ende, el primer período, o sea luego de la primera conflagración, mostró un momento peculiar del mundo occidental, en el cual se recurrió a medios poco ortodoxos o alejados de los viejos principios para encarar la regulación de la sociedad, o yendo a lo práctico, resolver las cuestiones litigiosas que se presentaban. En el segundo, hubo cierta contemporización

26 Morello (1984), en Diario de la Revista La Ley del 22 de agosto de 1984.

27 Bielsa (1964-1966) 580/1.

28 Lagarde (1934) 164; Bremond (1927); Saleeby (1908). 
entre la política y la judicatura. Lo reflejó un autor que citaremos varias veces, Gastón Federico Tobal, quién afirmó que el máximo Tribunal había acompañado la política gubernativa "para colaborar en la salida de crisis sociales y económicas», ${ }^{29}$ y específicamente lo señaló respecto a la llamada «Corte de Repetto», así denominada por el apellido de quién fue el presidente de la misma durante la década del '30 y hasta su renuncia, en 1946.

\section{Crisis v. espíritu nuevo}

La modernización tradujo el espíritu nuevo que gobernó no solo en la cultura sino recaló en el panorama jurídico. El mismo se reflejó en cuestiones puntuales tales como la integración del orden jurídico con un principio fundamental, la obtención de «lo justo». ${ }^{30}$ La plenitud de ese orden requería que las fuentes tuviesen el sostén de la justicia, pero una justicia encarnada en la satisfacción o el cuidado de lo que llamaban lo «social». Si bien no puede negarse que la justicia era el fin del derecho, la realidad se daba muchas veces de bruces con ese principio. El espíritu nuevo exigía que el derecho se remozara con una consagración de esta idea: lo justo, que ostentaba un grado más íntimo, informal, carnal, que el principio abstracto de justicia. Lo justo era alcanzar en puridad «la justicia». Las normas podían ser lógicas y razonables, pero al aplicarse aparejaban, en ciertas ocasiones, que sin desearlo o sin tenerlo en cuenta, se lastimase a una de las partes. De allí que lo justo era la concreción del principio de justicia, con una mirada más subjetiva y conectada con la realidad social. Quizás alguno podría equipararla a la equidad, pero, en verdad, no era preciso recurrir a este instituto, pues hubiese implicado reconocer que el ordenamiento obstaba a la citada concreción y había que recurrir a un atajo.

Uno de los principales referentes de ese espíritu nuevo estuvo encarnado en Alfredo Palacios, quién fue promotor de lo que llamó «nuevo derecho», es decir, el derecho que atendió las relaciones de trabajo desde la óptica de la protección de quién ponía al servicio de otro su fuerza laboral, y más tarde el emblemático título fue el que coronó uno de sus trabajos más significativos (1920). ${ }^{31}$ Fue autor de dos leyes fundamentales del período anterior, la ley de

29 Abasolo, Leiva, Pugliese (2000) 461, 464; Bidart Campos (1982).

30 Pugliese (1999). Sobre modernización Mossa (1930).

31 Palacios (1920).

$94 \quad$ Maria Rosa Pugliese 
descanso dominical y la reglamentación y protección del trabajo de mujeres y menores, y se ocupó asimismo de la defensa de la mujer contra la explotación sexual, a través de la ley que lleva su nombre y data de 1913. Ello no le impidió llegar a ser Decano de la Facultad de Derecho de Buenos Aires, hasta 1930, y más tarde dedicó sus esfuerzos a la Facultad de Derecho y Ciencias Sociales de la Universidad de La Plata.

Los tres elementos fundamentales del proceso: «espíritu nuevo», «la imperiosidad de la reforma legislativa», «El nuevo rol del Estado»

De lo que venimos exponiendo resulta que hubo tres elementos fundamentales en el proceso en estudio: el «espíritu nuevo», «la imperiosidad de la reforma» y un nuevo espacio para el «Estado», al que se agregó, a su turno, con moderación pero en forma gradual y continua, el nuevo papel de los jueces. Véase, en éste sentido, cómo reflejaba un juez y jurista como Gastón Tobal ese nuevo rol: «la vida, con su ajetreo incontenible, produce siempre cambios, que es menester armonizar de nuevo». ${ }^{\mathbf{3 2}}$

El espíritu nuevo expresó, entonces, lo que podemos definir como aires renovadores en una atmósfera viciada que requería soluciones que ya no podían brindar los viejos conceptos. Si la autonomía de la voluntad había sido la generadora de una política jurídica individualista, el crepúsculo de la voluntad privada se corporizaba en teorías tales como la imprevisión, la lesión, el enriquecimiento indebido, todas ellas guiadas por un principio de «impersonalización». Tal como advertimos, el individuo perdió terreno frente a formas colectivas, y el Estado asumió un papel directriz; obligaciones bancarias e hipotecarias quedaban bajo su órbita, se modificaban los precios mínimos y máximos, se obstaculizaban las rescisiones de locaciones urbanas y rurales, se sujetaban los contratos a reglas fijas establecidas por órganos estatales. Ahí nació una fórmula «contrato de adhesión», que obviamente era lo contrario de lo que había caracterizado a esta figura. Otro fenómeno íntimamente conectado es el de la economía dirigida, pues resultaba consecuencia de la intervención legislativa o judicial. La exaltación de lo social, para algunos, llevaba a la inseguridad, al desorden, porque no podían despojarse de una visión individualista del derecho. De esta manera lo visualizó

32 Tobal (1941). Ver párrafos medulares en Pugliese (2007), y Abasolo (2008) 23 y ss.

La «crisis del derecho» 1920-1940 
a posteriori el jurista Marco Aurelio Risolía al estudiar el tema, y marcó la disidencia que anteriormente habían exhibido Lafaille, Salvat y otros. ${ }^{33}$ Los países europeos habían entrado en su mayoría en esta tónica del dirigismo, y un ejemplo importante fue el de Francia.

Ese espíritu nuevo se manifestó en lo que puede definirse como la «imperiosidad de la reforma legislativa», otorgándole al derecho un nuevo sentido con valor social y además propiciando la renovación en todos los ámbitos jurídicos. La reforma, según los autores, no sólo era necesaria, sino debía ser inmediata, pródiga de expectativas para ocuparse de un «mundo nuevo». La adjetivación, denota la urgencia de la cuestión. La nueva realidad debía ser contemplada por una normativa adecuada y los tiempos eran acuciantes. Cabe apuntar, desde el mirador del siglo XXI, que tal planteo era innecesario, si nos atenemos a la realidad de que no hubo nuevo código o reforma y sin embargo, el derecho se «aggiornó». Puede constituir una de las tantas paradojas que nos ofrece el derecho o la historia del derecho. Aquél brotaba por otros innumerables caminos, pero los cultores se aferraban en parte a ese tipo de norma conocido. La lectura que podría hacerse es que no eran tan «modernos» como pretendían serlo. La imperiosidad podía satisfacerse por otros mecanismos, y así acaeció en muchos casos.

Reflexionando sobre el tema, se observa que al mentar la modernización, se hace mérito sobre la necesidad de cambios, la urgencia de una vuelta de página. Fue una manera asaz revolucionaria para ellos de afrontar las novedades, de responder a la situación del mundo en que se vivía. Podemos retornar a la figura del mundo del «ayer»y del «hoy». Para los juristas de posguerra, ya no servirían las modificaciones, grandes o pequeñas, sino debían traducirse en algo espectacular, que hiciera olvidar para siempre el mundo de «ayer» y todo su significado. Hay un nuevo protagonismo: la necesidad de «hacer» frente a la perplejidad de las situaciones nuevas.

Hubo cierta fiebre por la reforma, que estaba en los autores y se transmitió a la propia actividad gubernativa. El gobierno fue quien la canalizó, en 1926, a través de un decreto que designó la comisión redactora del código civil, cuya tarea era la revisión de la obra de Vélez Sarsfield ${ }^{34}$ para ponerla en

33 Risolia (1958) 183 y ss., contradiciendo a Lafaille (1925), Salvat (1938a) 347; Salvat (1938b) 51. Una aplicación de la misma la podemos encontrar en una causa anotada por Anastasi (1928).

34 Autor del Código Civil Argentino.

96 Maria Rosa Pugliese 
sintonía con la época. Se hablaba mucho de que el Código se había dictado cuando no había luz eléctrica y otros muchos adelantos. Pese a ello, es evidente que no es preciso que un código se refiera directamente a la electricidad para regular su uso como energía y en fin, no solo la actividad eléctrica en general, sino muchos otros descubrimientos devenidos desde 1871.

La mayor preocupación se asentó sobre la reforma del Código Civil, así como previamente se lo había hecho con el Código Penal. Aquélla fue recogida por la Revue Trimestrielle de Droit Civil (enero-marzo de 1937) tal como lo comprobó Leónidas Anastasi y lo dejó reflejado en la Revista La Ley. ${ }^{35}$ Según la revista francesa, se había tratado de obtener una legislación menos individualista, y tomando como referentes a los códigos alemán y brasileño, el proyecto contaba con una parte general sobre las personas, los bienes, los hechos y los actos jurídicos. En el ámbito del derecho de familia, se estableció la igualdad de los esposos, sin perjuicio de cierto predominio todavía a favor del marido, ya que era quien fijaba el domicilio conyugal y debía dar su autorización para que la esposa ejerciese una profesión. Los hijos adulterinos estaban en el mismo grado que los hijos naturales. A su vez, admitió la adopción. En el campo de los derechos reales, v. g., se reducía el plazo para la usucapión a veinte años, entre otras cuestiones. ${ }^{\mathbf{3 6}}$

La política jurídica argentina sufrió modificaciones por variadas razones y aunque hubo una predominante influencia del viejo continente, no omitió una mirada hacia América, y a su turno, llevó su influjo a sus países. Se debe tener presente que la contraposición entre Estados Unidos y la Argentina, hizo de ésta un norte a tener en cuenta por sus vecinos y también en otros

35 Anastasi (1938b) 12. Leónidas Anastasi remite a la Revue Trimestrielle, que en la p. 85, expresaba «el gobierno argentino ha constituido en 1926 una comisión poco numerosa compuesta de juristas particularmente calificados para revisar el código civil promulgado hace tres cuartos de siglo. A consecuencia de un anteproyecto preparado por Bibiloni, fue puesto en discusión, teniendo en cuenta la doctrina, jurisprudencia y el derecho comparado, inspirándose menos en el individualismo y más en los intereses de los terceros ...», señala que no puede seguir en detalle una obra tan importante, pero entre otras cosas afirmaba que «A ejemplo de los códigos alemán y brasileño, el proyecto comprende una parte general (art. 322) ...».

36 Sobre el tema de la reforma legislativa, resulta de interés el trabajo de Diaz Couselo (2007) y los textos seleccionados de Enrique Martínez Paz, Alfredo Colmo, Juan Antonio Bibiloni, Juan Carlos Rébora, Héctor Lafaille, Gastón F. Tobal, 231 y ss. 
países más alejados. Esa unidad material y espiritual con la vieja Europa, no dejó de influir en las referidas novedades.

Sin dejar de lado el iuspublicismo, todo lo contrario, cabe apuntar que en la Argentina y en Uruguay se conformó una fuerte corriente iusprivatística y iusprocesalista, que, con sus diversos matices, se reflejó en los proyectos de reforma o de nuevos códigos civil y procesal, y en las modificaciones del código comercial, así como en el campo del derecho del trabajo que, a partir de la actividad de los juristas de la denominada por Víctor Tau Anzoátegui, «generación de 1910» y luego con la «generación de 1925», intentaron producir una modernización en dichos sectores. Dentro de esa corriente iusprivatística no puede omitirse un fuerte componente publicista, ya que el sentido social que caracterizó el momento, debía salir del margen que le brindaba un código fruto de un liberalismo más dogmático.

En el orden que venimos exponiendo, en esta incursión sobre la modernización argentina, resulta útil la lectura de la literatura jurídica del período, y también detenerse en las revistas jurídicas, porque ofrecen la experiencia europea y latinoamericana, además de la argentina. En este observador de la realidad jugó papel estelar el citado Anastasi, quién con la colaboración de Lorenzo Carnelli en Uruguay, trasuntó en las revistas Jurisprudencia Argentina y La Ley, - fundadas por Anastasi -, la vida jurídica continental, ya sea a nivel de doctrina, jurisprudencia de sus tribunales y de legislación. Esas revistas, además de reflejar los cambios jurídicos, sea en la doctrina, legislación y jurisprudencia europea y americana, pusieron de relieve a la fuente jurisprudencial como una aliada insospechada de la modernización.

El derecho extranjero, en la concepción de Anastasi, servía para iluminar la doctrina y la jurisprudencia, inspirando soluciones alcanzadas en otros países, y a su vez, sirvió como correlato para generar otras, que terminaron por imponerse en el ámbito americano. Ese papel transformador se continuó incluso tras su desvinculación de la Revista de Jurisprudencia Argentina, y en el caso de La Ley, tras su prematura muerte. Constituía un faro que iluminaba lo jurídico. La revista como tal podría suponerse que tiene una resonancia relativa, pues como toda obra periódica, ofrece notas de fugacidad contingente, pero he ahí una fuente donde abrevar para conocer el pensamiento jurídico-político de una época, sus preocupaciones y soluciones. Por ello, si alguien desea conocer la modernización del derecho en la Argentina, no puede soslayar el estudio de sus revistas; tras la anotación de sentencias, artículos doctrinarios y legislativos referidos a la Argentina cuanto a los 
países extranjeros, asomaban las nuevas teorías, los cuestionamientos a las viejas estructuras, etc, todo lo cual traducía el espíritu nuevo a que hemos hecho referencia. ${ }^{37}$

Los prólogos de las dos revistas argentinas que hemos referido, exhibían con total naturalidad y gráfica expresión el pensamiento jurídico de un hombre de avanzada, pero al mismo tiempo, eran el reflejo de aquello que él pulsaba en la realidad y deseaba dejar sentado en sus páginas, así como la opinión de muchos otros juristas contemporáneos. Hoy constituyen fuentes indiscutibles para conocer el período en estudio y los elementos que hemos diseñado. Rememoraba Anastasi en el Prólogo de 1931 de la Revista de Jurisprudencia Argentina, el anterior de 1924, donde había concluido en una síntesis inigualable:

«Quedarán satisfechas nuestras aspiraciones si hemos contribuido a difundir las enseñanzas de nuestros jueces y de nuestros escritores. Buena o mala, lucida o mediocre su obra, acumulamos materiales para la historia del derecho argentino. Y el crítico de las edades futuras dispondrá de elementos suficientes para trazar las líneas de la evolución de un pueblo progresista, que ha oscilado en su cultura jurídica entre lo arcaico de las viejas formas y las concepciones inquietas y atrevidas del derecho nuevo».

Y continuaba, en referencia a la jurisprudencia:

«Sea que ella interprete la ley, sea que ella decida en el silencio de las leyes y para llenar sus lagunas, la jurisprudencia pronuncia decisiones que se ejecutan; es ella la que representa la forma viva del derecho, ella sola es la regla aplicada ... prestigiamos la fuerza creadora de la jurisprudencia ... Discusiones tan eruditas como las que se produjeron en la Cámara Civil primera ... demuestran que nuestros jueces no convierten en ídolos sus propias resoluciones. Con la publicación de nuestra colección ... aspiramos ... a que ella confirme la fórmula sintética que enseñaba Carlos Octavio Bunge, en su inolvidable curso de Introducción al derecho: «el derecho es la vida»".

A su turno, en el Prólogo del primer Repertorio de La Ley, en 1938, acotaba que seguiría el rumbo marcado en la Revista de Jurisprudencia Argentina, para que la misma fuese «un órgano eficiente del estudio de nuestra jurisprudencia, señalar su fuerza creadora ... asegurar la necesario vinculación

37 Sobre el particular me he interesado, ver Pugliese (2007), y específicamente Pugliese (2008). Asimismo Tau Anzoátegui (2011) 108-110, cita el resumen de trabajo de mi ponencia sobre «Catalogación y comentario de las notas de Leónidas Anastasi en la Revista La Ley, 1936-1939», en XXII Jornadas de Historia del Derecho Argentino, 2008, aún no publicado. 
entre el foro y la Universidad ... haremos frecuente referencia a la evolución jurídica, política o social de los pueblos hermanos de América, así como del derecho europeo ...». Recordaba los auspiciosos juicios que le dedicara el Instituto de Derecho Comparado de Lyon, señalando el mérito científico que trasuntaban sus páginas, y en otro orden, destacaba el criterio «humano y equitativo» de la interpretación judicial de la ley de accidentes de trabajo. Advertía que «el legislador se mueve muy tardíamente entre nosotros, en gran parte por defectos de nuestra organización parlamentaria ... Los jueces han superado a la ley. Sobre las normas básicas de aquélla se ha asentado un verdadero derecho pretoriano, con el que se ha subsanado parcialmente la inexplicable dilación de la reforma de la ley...», y trasuntaba que así se hubiera ajustado al ritmo que exigía la cuestión. ${ }^{38}$

En un artículo dedicado al nuevo régimen de familia en el proyecto de reformas del Código Civil argentino, Gastón Federico Tobal se refirió al trabajo de revisión del código vigente, ordenado por Decreto del Presidente Alvear y su ministro de Justicia Antonio Sagarna, catalogándola como una obra indispensable. Además, en artículos publicados en el diario La Prensa y reunidos en un folleto, demostraba lo que llamó como tantos, la necesidad imperiosa de la reforma, - resultando así para los investigadores una fuente fecunda de conocimiento epocal -, al expresar que los códigos,

\begin{abstract}
«por más perfectos y amplios que sean, solo representan la exteriorización del derecho en un momento de la evolución de los pueblos. Ellos revelan la forma en que el ritmo que permite la convivencia, se muestra en esa hora, pero los intereses, las pasiones, las necesidades económicas, y la vida en fin, con su ajetreo incontenible producen siempre cambios que es menester armonizar de nuevo. El nuestro, pese a sus defectos y a sus años, será siempre una obra valiosísima que colocará al Doctor Vélez en un sitial, más el brusco cambio de la post-guerra, las modificaciones en la economía y en el trabajo y hasta en cierto modo, la limitación que el mismo Vélez creara a la obra reformadora de la jurisprudencia, atándola con preceptos que importaron vallas difíciles de franquear, el espíritu individualista de su época que trasciende en todo su articulado y el excesivo acatamiento al sistema de las libres convenciones, todo ello, unido a la falta de un Tribunal de Casación, que hubiera permitido fijar la norma interpretativa, son los motivos que han acortado la vida, empero larga de su código y han impuesto la necesidad de una reforma que adecue las reglas jurídicas a las exigencias del momento, aligerándolas de principios demasiado absolutos, del límite que importa a las definiciones a que fuera tan afecto el codificador y traiga a la obra jurídica más importancia para la vida nacional, ese
\end{abstract}

Abasolo (2008). 
espíritu nuevo que nos reclama el deber ético olvidado en el código, el amor hacia el prójimo, y con él, derechos que no vean un campo opuesto y cerrado, en el terreno de la moral y de la cooperación.» 39

Si nos retrotraemos a un jurista del siglo XIX, Lisandro Segovia, éste afirmó que el Código se había inspirado en el francés de 1804, y por ello sus comentaristas obraron como los viejos exégetas. Como código expresaba el pensamiento de Mayo y el de los constituyentes de 1853, y así lo caracterizaba como un código liberal, racionalista, dogmático, ahistoricista, con un sustrato económico. ${ }^{40}$

¿Qué causas determinaron las nuevas orientaciones en la legislación? ¿Había cambiado el concepto mismo de derecho?

La experiencia nos muestra que la vida se animó por el nuevo espíritu que exigía, para establecer normas nuevas o adaptación de las vigentes para atender esa situación.

Resulta interesante seguir el pensamiento de Tobal, porque nos evita efectuar demasiadas reflexiones, y además, las expuso este jurista con elegancia y notorio conocimiento.

Se preguntaba si había una mutación en el concepto mismo del derecho, cuáles eran las causas que determinaban las nuevas orientaciones, si la vida estaba animada de un nuevo espíritu que exigía cambios jurídicos. Sus interrogantes en realidad eran proposiciones para desarrollar asertivamente. De ahí que expresara que los fenómenos que traducía el nuevo siglo, no podían sino trasladarse a la ciencia jurídica. Para ello recurría a Josserand, quién había expresado que «el derecho estaba entre el ideal moral y el materialismo económico, y los signos de la época son la colectivización y la mecanización, sin personalidad, sometida al imperio de la velocidad que puso de relieve Strovsky». ${ }^{41}$

Reconocía como su citado, que la mecánica y la economía habían efectuado transformaciones vitales y destacaba la forma en que la última se había proyectado en el ámbito jurídico. En el propio concepto de Estado y la situación del individuo frente al mismo, que había generado un intervencionismo estatal en la economía, y que permitía advertir una posición inter-

39 Tоваl (1936). Con «Mayo» se hace referencia a la revolución del 25 de mayo de 1810 en Buenos Aires, es decir, el comienzo de la ruptura con España.

40 Segovia (1874), en Risolia (1958) 182 y ss.

41 Josserand (1930); Tobal (1936). 
media entre individualismo y socialismo, producto de una nueva realidad social. Mostró cómo se añoraba la anterior seguridad, pero la realidad era que, como corolario se había producido una centralización política, que evidenciaba el retroceso de las autonomías locales frente al poder federal, y surgía una legislación de emergencia, que era el reflejo de necesidades colectivas súbitas y que se suponía que el Estado debía atender.

Fue evidente la influencia del aporte de Geny, con su célebre obra Méthode ${ }^{\mathbf{4 2}}$ en el pensamiento europeo y también quizás de manera indirecta en el argentino, dando un gran valor a la apertura del juego de las fuentes jurídicas, y en particular reconociendo la interpretación del juez, pero con cierta duda respecto a la jurisprudencia como fuente formal, sin perjuicio de admitir su autoridad para el uso de los intérpretes del derecho. Algunos doctrinarios argentinos tuvieron sus prevenciones respecto al valor cuasi legislativo de la jurisprudencia, tal como puede comprobarse en Rodolfo Rivarola ${ }^{43}$ quién dijo que no era posible reemplazar la ley por lo que llamaba la costumbre de los tribunales. Pero esta resistencia fue luego vencida por la realidad de los hechos, ${ }^{44}$ y así se puede observar en Alfredo Colmo, Héctor Lafaille, Luis Méndez Calzada, Leónidas Anastasi. ${ }^{45}$

¿Qué proponían y citaban los juristas «modernos»? Se apoyaron en el derecho comparado, tanto para buscar los cambios como para dar fundamento a alguna conclusión doctrinaria, aunque, tal como lo afirmó Enrique Martínez Paz «no se tenía una idea clara de éste», pues en el Congreso Internacional de Derecho Comparado de París de 1900 figuraba en el temario la definición y el método de aquél, y se reiteró en el Congreso de La Haya de $1932,{ }^{46}$ pero se la tenía como una exigencia de la época. Implicaba la consagración de la unidad de los principios jurídicos y a su vez, decía Martínez Paz, el método comparativo era útil para tomar en cuenta los antecedentes locales en primer término. Como ejemplificación citaba las obras de Domat y de Pothier en Francia; el derecho alemán, en general y la obra del

42 Geny (1899), en castellano 1902.

43 Rivarola (1910).

44 Puede comprobarse en el debate de la ley 7055 de ampliación de la justicia en Buenos Aires, Diario de sesiones de la Cámara de Diputados, Congreso Argentino (1910), T. 1, en el cual se destacaron los juristas Manuel A. Montes de Oca y Manuel B. Gonnet, ambos diputados.

45 Pugliese (2007).

46 Martínez Paz (1934). 
codificador suizo Huber, que se realizó con la unificación de los derechos locales como antecedente para la conformación de su derecho nacional. En segundo lugar, luego de haber comparado las normas internas, aparecía el derecho comparado externo, es decir, apuntando a otros derechos nacionales. Tal la tarea de Vélez Sarsfield, quién redactó el Código argentino teniendo presente los demás derechos positivos de Europa, y de allí su afirmación en cuanto a que el código era la expresión de la ciencia jurídica moderna por haber considerado la legislación extranjera más adelantada. Llegaba a la conclusión de que el derecho comparado encontraba el fondo común que había en los países correspondientes a un mismo sistema. ${ }^{\mathbf{4 7}}$

Superado el positivismo normativo, adorador del texto legal, se entró en un proceso donde el derecho se transformaba en realidad. Un nuevo adagio lo acompañaba: al juez le correspondía la noble tarea de «humanizar el derecho, con eso se transforman progresivamente las instituciones». La vida del derecho se comenzó a percibir, por muchos, a través de las sentencias. Se agregaba que su función y su aporte y auxilio a las novedades era que auscultaban la realidad social como ningún otro protagonista.

Del planteo reformista surgió el anteproyecto de reformas al Código Civil, obra de Juan Antonio Bibiloni. Este se mostraba poco receptivo de cuestiones tales como el derecho natural, para concluir que las disposiciones eran siempre exclusivamente jurídicas. Ni hacía falta que interviniesen elementos ajenos como la equidad, ni otras consideraciones, porque el derecho no era un conjunto de disposiciones arbitrarias y caprichosas, ni reñidas con la justicia, sino un orden congruente y lógico emanado de la realidad.

También vale analizar el concepto del Estado ${ }^{48}$ y sus fines en ese momento. Había nuevos principios, otras orientaciones que abrían una ventana vedada hasta entonces, para dar una bocanada de aire en un ambiente que se sentía ahogado por el mantenimiento de pautas que ya se vivenciaban como vetustas. No puede llamar la atención que, tras graves situaciones de orden económico y social de origen mundial y que ofrecía su correlato en nuestro país, la década del '30 exhibiese un profuso intervencionismo eco-

47 Sobre el código civil y sus fuentes, ver Cabral Texo (1919); Colmo (1927); Juicios críticos sobre el proyecto de código civil argentino (1920) 160, 170 y ss. En este último en particular es interesante observar la famosa polémica entre Dalmacio Vélez Sarsfield y Juan Bautista Alberdi sobre aquél cuerpo.

48 Capograssi (1921); Capograssi (1941a); Grossi (2011) 65 y ss., y especialmente 217 y ss. 
nómico del Estado, y el concepto del derecho planteaba una situación intermedia entre el individualismo y lo social. Por sobre todo, la realidad social se impuso, añorando, podría decirse, el equilibrio que derivaba de la seguridad, de la estabilidad. Políticamente se consolidó una centralización a este nivel, y los poderes federales aparecieron robustecidos en detrimento de las autonomías locales, que como lógico efecto, implicó una pérdida de espacio. Junto a ese planteo interventor del Estado, en forma paralela, se configuró una legislación de emergencia que fue creciendo en forma desmedida, quizás tergiversando el sentido de este concepto, pues el mismo suponía una necesidad colectiva, súbita y grave, que no podría configurar una dimensión extensiva que fuese más allá de estos criterios básicos.

Si estudiamos la situación en la Argentina en el contexto americano, no puede soslayarse la evaluación de las estructuras sociales y las instituciones políticas, tal como lo planteó Jacques Lambert en $1963^{49}$ y lo había hecho con antelación Ortega y Gasset. ${ }^{50}$ Ese análisis muestra distintos niveles culturales y económicos, desigualdad de recursos naturales y sociales. En su mayor parte, según esos autores como producto del proceso de colonización castellana. América fue el resultado de temores y proyectos casi utópicos. Por un lado decía Lambert, estaban la fatiga, la nostalgia, el hambre, las enfermedades, en fin, la incertidumbre ante lo desconocido y, en una simbiosis curiosa, el espíritu de servicio, el afán de honra y a la vez la sed de oro, el aliento religioso que movía a los hombres que llegaban al continente.

El proceso fue urbano, pero no puede omitirse la consideración de la vida fuera de las ciudades, que ofreció características importantes a tener en cuenta en la etapa post española. En menos de un siglo observamos el asentamiento y poblamiento y la consiguiente organización, aunque quedaron amplios vacíos reales y otros fuera del alcance de la autoridad, desarrollando una experiencia local tan rica en autonomía como no podría suponerse tras la coraza virtual de un imperio. Los autores que seguimos nos refieren un ansia de libertad que se repitió en círculos concéntricos. Si los criollos mostraron ese fenómeno con relación a los europeos, otro tanto sucedió con los indios, los mestizos y mulatos frente a los criollos y europeos. Asimismo los virreinatos frente a la metrópoli; las capitanías generales y otras demarcaciones delante de los virreinatos. Ese juego se mantuvo en los nuevos

49 Lambert (1939).

50 Ortega Y Gasset (1925).

104 Maria Rosa Pugliese 
países que se consolidaron tras la ruptura del imperio. A la aparente unidad del gobierno común metropolitano, se sobrepusieron las diferencias e incluso los antagonismos y rivalidades entre esas antiguas provincias. La necesidad de la guerra hizo del ejército un elemento de poder con el cual había que contar para gobernar.

En el caso argentino, como también podría incluirse a Uruguay, la fuerza arrolladora de la inmigración masiva europea provocó cambios estructurales, generados por una elite que buscaba cambiar el rostro al país, pero a quienes luego esa misma fuerza los desplazó paradójicamente, conformándose partidos políticos que respondieron a esa nueva fisonomía, el radicalismo y el socialismo y conjuntamente se dieron otras organizaciones, ya sea sindicatos, cooperativas, etc.

Esas mutaciones sociales aparejaban una reacción sobre los derechos individuales de raíz individualista, y así de una libertad e igualdad virtual, declamadas pero a veces poco aplicadas, se trató de lograr su concreción social. De esta manera, sufrieron variaciones principios e institutos tan arraigados como la autonomía de la voluntad y el contrato. Apenas deslizaremos en este trabajo algunas consideraciones sobre el tema, pues resulta necesario dedicarle una investigación profunda y más amplia, que aquí no cabe, teniendo en cuenta que hemos privilegiado la materia de la crisis en general.

Yendo a los principios generales, debemos ocuparnos en primer lugar de la interpretación, que cada vez fue más extensiva por obra de la jurisprudencia de los tribunales.

Al prologar en 1932 la obra de Giorgio del Vecchio denominada «Los principios generales del derecho» - leída y analizada entre nosotros -, Clemente de Diego, puso en evidencia de manera clara y acertada el pensamiento del filósofo italiano. ${ }^{51}$ Afirmaba allí que un código de espíritu viejo, por mucho que se renovase, no satisfacía las ansias de espíritu nuevo que sobrevolaba el derecho civil. El intérprete en este caso, debía estar atento más que a su propio concepto de lo «justo» y del «derecho», a los signos objetivos que provenían de la concepción de la época y del propio país de que se tratase, y ello involucraba a las distintas fuentes del derecho y a los principios generales.

Cuando el intérprete era el juez, debía reflexionar, pues la ley no daba todas las soluciones e incluso, reconocía que en muchos casos ésta guardaba

Del Vecchio (1948); Del Vecchio (1938).

La «crisis del derecho» 1920-1940 
silencio o era insuficiente, y allí entonces entraba a jugar su rol, que era el de llenar ese vacío. Como ejemplo citaba los códigos francés, belga, alemán, donde ese silencio debía ser suplido mediante la ciencia, por la conciencia del juez. En tanto, otros códigos como el austriaco, el portugués, el italiano, y en América los de Méjico y Perú, ofrecían ciertas claves, tales como los mentados principios generales, y la analogía principalmente. Incluso el código de Austria se refería al derecho natural. Al preguntarse cuál era su significado, algunos autores como Zeiller, Nippel, Schuster, Berger remitían a la escuela de Derecho Natural, pero otros hablaban de la naturaleza de las cosas, señalando las fuentes romanas, la idea de derecho, es decir, rechazaban la referencia a elementos filosóficos. Mientras Unger, además de la analogía, aceptaba la actividad creativa del juez. En cuanto al código italiano, dio pautas para la búsqueda de las soluciones: así marcaba la ley, la analogía, los principios generales y los autores se habían dedicado a explicar a cada uno de éstos.

Se alejaba Del Vecchio de la doctrina que apuntaba que el derecho romano y el común, constituían principios generales y buscó, en la filosofía del derecho natural, la función supletoria de las normas del derecho positivo. La analogía, a su turno, no excluía otros principios generales. Pero, la interpretación analógica no se podía extender indefinidamente, y así cuando no era aplicable, se debía recurrir a otros principios generales. Ambos conceptos, así, se integraban.

Frente a la omnipotencia de la ley, recreaba a la costumbre como fuente del derecho. Apoyándose en Savigny y Puchta, afirmaba que el derecho positivo tenía en su interior una elasticidad y una energía orgánica que le permitía adecuarse a las cambiantes necesidades sociales. En su argumentación brindaba el siguiente orden de prelación: en primer lugar había que recurrir a la ley, y cuando ésta callaba, se debía recurrir a los principios generales del derecho y ahí entonces hacía jugar a la costumbre, pero en el caso de no hallar tampoco allí la solución, recurría a «la propia realidad social investigada con criterio científico, iluminada, por los principios de la razón y la justicia. Para ello el intérprete goza de libertad». ${ }^{52}$

Al comentar al filósofo italiano, De Diego decía que al recurrir a los principios generales evadía la arbitrariedad subjetiva, basándose en un requi-

De Diego (1932). 
sito esencial como era la congruencia del orden jurídico. Ese orden se basaba en un sistema que tenía por base a la Constitución y sus principios, considerando que en tales principios había una «ratio civilis», no podía recurrirse a principios contradictorios con la legislación, pues lo único que lograría sería atacar la certeza y con ella la seguridad jurídica. Sin embargo, el autor dio un paso avanzado, pues aseveraba que la ley no podía desconocer la jurisprudencia del Tribunal Supremo, otorgándole así el carácter de fuente del derecho.

Como ejemplificación de un código novedoso, pero que respetaba la integralidad del sistema jurídico, hacía referencia al Código Civil suizo de las obligaciones, que en su orden de prelación le indicaba al Juez actuar como si fuese legislador, si no encontraba una solución legal o consuetudinaria. Podría suponerse que con ello se le brindaba un exceso de libertad; sin embargo, marcaba que debía inspirarse en la doctrina y en la jurisprudencia. Estos constituían un obstáculo para adoptar criterios de tipo individual y subjetivos.

En definitiva, el magistrado, al ejercer su función, no debía modificar las leyes, sino justamente tener en cuenta su espíritu. Reiterando un concepto que ya hemos esbozado, el juez debía precaverse de buscar «lo justo» de acuerdo a su criterio subjetivo, sino hallarlo en el ideal social que provenía del pueblo. Por sobre todo rescataba dos derechos fundamentales: la libertad y la igualdad, que en su intrínseca naturaleza eran principios dinámicos.

En la Argentina, cabe analizar la temática por medio de una voz autorizada como la de Risolía. En su opinión ${ }^{53}$ aunque el código civil contemplaba en los artículos 15 y 16 criterios de interpretación, los mismos no estaban sistematizados como aparecían en el Code francés. En tanto, el Código Comercial los reunía en los artículos 217 a 220. Por ello la jurisprudencia cubrió ese vacío, pero en tanto el jurista reconocía que los códigos no agotaban el derecho, señalaba que el artículo 1198 del Código Civil favorecía el extenso poder del magistrado y en la época estudiada esa situación se estaba consolidando.

Para este jurista, entonces, el papel del juez de acuerdo a las codificaciones del siglo 19, halló una limitación de las atribuciones interpretativas ante el texto expreso no sólo de la ley sino del acuerdo privado. Allí se detenían sus facultades. La ley solo lo armaba con el recurso de nulidad, pero para casos 
específicos, ya sea donde estaba comprometido el orden público, la moral, las buenas costumbres, la protección de incapaces. Esos límites eran infranqueables. «Hoy en cambio, - decía, cuestionándolo -, se usa». La imprecisa latitud de ese poder le hacía argüir sobre semejante facultad que extralimitaba el orden jurídico y lo subvertía, implicando un riesgo para la seguridad jurídica. Tras la fachada de la cuestión social se dictaban leyes sobre moratorias de las obligaciones, bancarias, hipotecarias, se acordaban prórrogas a las vencidas, se modificaban los términos, ya sea en lo atingente a los precios, causas de rescisión de locaciones, etc.

Luego de superar el concepto de que el juez estaba inexorablemente ligado o bajo el mandato de la ley, doctrina expuesta por la escuela de la exégesis y retomada más tarde por los positivistas legalistas, ahora se llegaba a decir que el juez percibía mejor la realidad para hacerla efectiva. Y no olvidemos que la etapa estaba marcada por el signo de la «realidad». No obstante, tampoco se adoptaba el principio de que el juez se pudiera convertir en legislador. Eso ponía al juzgador en una situación quizás harto incómoda. Por un lado se le decía que nadie mejor que él para auscultar la esquiva o frontal realidad, y para resolver en consecuencia, y por el otro se le planteaba el dilema de la división de poderes y los límites entre la labor legiferante y la judicial, como un atalaya cercado por un foso lleno de leones. Claro que cuando el juez resolvía lo hacía para un solo caso, su creación era unidimensional ... pero también era evidente y no se podía negar su efecto: la doctrina que emanaba de un fallo que atendía la realidad, era reconocida, a partir de ella con un valor superlativo. Su adquisición en el articulado del proyecto de reformas, demostraba que lejos de no poder sortear el foso, lo hacía con más comodidad de la que se podía presumir y que casi nadie se espantaba por ello. ${ }^{54}$

Ya Duguit se había aventurado a excluir del campo jurídico la figura del juez autómata de Montesquieu, pues alegaba que el juez debía descubrir el pensamiento del legislador, auxiliándose con la realidad; estar atento a la visión del mundo, auscultar la realidad y también no detenerse inútilmente en interrogarse que había pensado el legislador, porque sería una tarea reducida, sino estudiar la sociedad en la que vivía. Ello, pues concebía la ley como un predicado de la constitución social, de la naturaleza del hombre y de la sociedad. Sin hesitación y a la postre advertía una verdad de Perogrullo: «hay

54 Justo (1937) 41; Aвasolo (2008).

108 Maria Rosa Pugliese 
más derecho que el escrito». Ni menos monopolio en la producción del derecho, aunque no llegaba a admitir que su interpretación debía ser libre para evitar equívocos. Tampoco era prudente el enfrentamiento entre la ley y el derecho, sospechando de la primera, pues se desembocaría en una anarquía.

Claro que Montesquieu había atendido a otro problema cuando ciñó al magistrado al texto de la ley: deseaba alejar al derecho de la arbitrariedad y los deseos de un hombre o de una casta. Pero, aunque actuase con mayor independencia, se le seguía obligando a sustraerse de las pasiones del momento. El principio novedoso fue que en estas décadas comenzó una orientación que sustentaba que debía inspirar sus sentencias en lo que puede denominarse «un sentimiento del derecho y en la conciencia jurídica social». Cuando la aplicaba, decía De Diego, en un texto muy feliz, «el juez la desarrolla, la aclara, la suple, la elabora, y perfecciona, la vivifica en suma, conforme las aspiraciones de la razón común y a impulsos de la sociedad histórica para que fue dada». ${ }^{55}$ Aquí entraba a jugar el papel de François Geny, y su mérito de haber resaltado las otras fuentes del derecho extendiendo el dominio dejado a la libre investigación científica.

Ello, pues el derecho se renovaba y se renueva todos los días, porque la ciencia jurídica debe corregir continuamente sus construcciones. La voluntad del legislador se desvinculaba de la ley desde el momento de su promulgación, luego quedaba y aún queda en manos de sus intérpretes.

Esa tarea de interpretación la hacía el juez quien debía hacerla vivir, pues el fin era la solución justa del caso. El fin del derecho, en esta concepción, era hacer justicia. No entraba en la tarea del juez buscar una interpretación «auténtica», sino justa. Claro que en los momentos dispuestos por este trabajo, el juez se vio obligado a atajar su desánimo por la decadencia del poder legislativo y la quiebra de la separación de poderes. Otra labor fundamental del juez, acotaba, era no politiquizar la justicia. Eran incompatibles. Comentaba lo que sucedía en el Tercer Reich. Los partidarios de aquella concepción iniciaron a principios de siglo una cruzada dirigida contra la opresión de la doctrina y los textos. Reivindicaron para el juez una gran libertad de apreciación. Amplias facultades para cubrir los vacíos legislativos, pero en su contemporaneidad, ellas se usaban con sentido político. 
El aumento del papel judicial traía aparejado, a su turno, una consecuencia no deseada, y era la de afectar la seguridad y la certeza que se esperaba del Derecho, y el juez se transformaba, puede decirse, en un árbitro y casi su único «vocero». El peligro era que ya no quedaba la solución en manos del iudex perfectus, ${ }^{56}$ sino de alguien que podía adueñarse de un espacio que hasta entonces le correspondía a la ley, como expresión de la voluntad popular en un régimen democrático, que recaía en sus representantes, y no en un funcionario que había sido designado sin la participación directa de aquélla. ${ }^{57}$

La presencia protagónica del juzgador se vio reflejada en la Argentina, y al efecto las revistas jurídicas del período receptaron la misma, pues al anotar fallos de nuestros tribunales o comentar otros extranjeros, dejaron traslucir esa labor interpretadora y hasta creadora. Un ejemplo de este nuevo modelo se puede observar claramente en un comentario efectuado por Leónidas Anastasi, en la revista La ley donde exhibió cómo el juez argentino podía llegar a atenuar el rigor de una cláusula penal, ${ }^{58}$ en contraposición a lo que sucedía con el juez francés, al referirse a un fallo de la Corte Casación francesa de París, del 7 de enero de 1936. Aquél trajo a colación una sentencia de la Cámara Civil Primera, con el voto de Sauze y Tobal, quienes establecieron, que, sin perjuicio del sistema de la libertad de las convenciones del art. 1197 del Código Civil, el propio codificador se adelantó a contrarrestar tal aparente criterio. Al efecto, recurrieron a las pautas que establecían los arts. 502, 530 y 953, que en esta ocasión y otras anteriores permitieron a los jueces morigerar una libertad excesiva, para contrarrestar una cláusula penal usuraria, logrando en la práctica lo que permitía el Código Civil alemán, al facultar a los magistrados a moderar las cláusulas que considerasen excesivas. El tercer juez, Doctor Grandoli, en su disidencia, aseveraba que el art. 522 del código consagraba la inmutabilidad de la pena convenida en las obligaciones. En su apoyo, sostuvo que el arbitrio judicial no era procedente y que la cláusula impugnada se atenía al art. 953, pues no era lesiva del orden público.

El comentarista elogió la decisión de la mayoría y opinó que la disidencia se apoyaba en un criterio excesivamente individualista que «contrasta con las

56 Martire (2005).

57 Pugliese (2010) 1267-1268. Pugliese (2006).

58 Anastasi (1938c). 
exigencias de ésta época de depresión. Este es el momento en que el derecho debe ejercer su función reguladora en el equilibrio social». Para ello, no solo recurrió a la exposición de sus argumentos e ideas innovadoras, sino se apoyó en Saleilles (Obligaciones, 343) y asimismo, y en la doctrina nacional, exaltó a Colmo, que se oponía a un desmedido rigor, catalogándola como una tiranía excesiva, que, sostenía, el derecho contemporáneo fulminaba. ${ }^{\mathbf{9}}$ Demostrando su dominio de la doctrina, señaló la discusión que se había dado en Francia, donde pese a las opiniones divididas, en definitiva prevaleció la solución actual. En Brasil, se puso freno a la libertad absoluta de las partes, inspirándose en un sentido de equidad. ${ }^{60}$ En cuanto al derecho soviético, se seguía la solución del Código alemán, pues se daba un control en las cláusulas penales.

También cabe traer a colación la visión sociológica y con ella la puesta en relieve de las transformaciones sociales inspiradas en las costumbres y la práctica, como motivadores de reformas. Otros destacan el fin social de la aplicación legal, con una interpretación teleológica, así como el sentimiento político que la envuelve.

La realidad social había adoptado la calidad de un poder transformador que actuaba sobre el derecho y también con más hondura sobre la jurisprudencia. Era un movimiento evolutivo y tenaz, quizás aluvional. Luego de su paso, el derecho quedaba remozado.

Por esto, la pesquisa de la voluntad del legislador era una tarea inútil, si lo esencial era la nueva realidad social, que podía hallarse indagando e investigando aquella voluntad. Todo legislador condensa aquello que le muestra la realidad o dicho de otro modo, todo lo que la situación social le imponía. En el mismo orden, quienes buceaban en los trabajos preparatorios de la ley usaban una brújula poco apropiada, de acuerdo a Joaquín Dualde, ${ }^{61}$ pues lo

59 Distinguió Anastasi la diferente posición de la Cámara Civil primera respecto a la segunda. La primera con el voto de Pera, Tobal y de Vedia y Mitre, rescató tres precedentes de primera instancia, concluyendo que se configuraba un enriquecimiento indebido a favor del acreedor, respecto a una cláusula excesiva, que no podía ampararse por la ley ni por la jurisprudencia, declarando su invalidez y reduciéndola. Ello, pues se podría llegar a una expoliación del deudor, y en virtud de ser contraria a los usos y costumbres. Anastasi afirmó que esta era la corriente más «justa», al inspirarse en aquellos fines que exige «la convivencia social».

60 LARCERDa (1936). Cita de Anastasi, ver nota 53, quién recuerda como el antiguo derecho portugués lo reconocía en una Ordenanza.

61 Dualde (1933) 154. 
acertado era insuflar un espíritu nuevo a la ley vieja. Tocaba al intérprete y no al legislador aclarar algún aspecto oscuro.

Ya Saleilles exigió que hubiese concordancia entre el derecho y la vida. Es decir, que las alteraciones y los cambios que no emanasen de causas espurias, el derecho las atendiese para no quedar retrasado en la atención de las cuestiones que preocupaban o interesaban a la sociedad.

Principios nacidos de la burguesía que trasuntaban el triunfo del racionalismo jurídico, consolidados luego de la Revolución Francesa, y como ejemplo liminar: el contrato era expresión de la libertad y la igualdad, así como del derecho de propiedad, y había conquistado un lugar de privilegio como resultado de la autonomía de la voluntad. La crisis de ésta, aparejó que la otra adquiriese un matiz endeble, perdiendo el valor que le había reconocido el Code.

En la concepción racionalista y liberal no había derecho sin libertad y viceversa. La pérdida del concepto clásico del contrato era producto de la transformación operada luego de la primera guerra mundial.

Las reglas jurídicas habían constituido en esa línea axiomas absolutos, que se bastaban a sí mismos. En un análisis sobre la evolución del derecho interesa justamente esa movilidad. La libertad y la responsabilidad eran dogmas de fe. Despojada de sacralidad, la vida social les otorgó ese tinte a otros principios y valores. Las novedades de la posguerra pusieron ineludiblemente en revisión los postulados excesivamente liberales. El sistema sobre esta base entró en crisis, pues los valores de otrora perdían vigor. La intervención cada vez mayor del Estado tanto en la vida jurídica como en los restantes aspectos, fue producto de una realidad más compleja y que avanzaba con una fuerza incontenible. Así las cosas, la voluntad individual cedió terreno a la voluntad estatal y social, y quedaron enfrentados por un lado el principio de la autonomía de la voluntad, con los principios de solidaridad, equidad, justicia social, orden público. ¿Se podían conciliar?, ¿hasta qué punto y con que resultado? Con este interrogante cerramos esta primera parte de una temática tan interesante, pues aspectos tan relevantes como la autonomía de la voluntad y el contrato merecen un tratamiento especial, que desenvolveremos en una segunda parte. 


\section{Bibliografía}

Abasolo, Ezequiel, David Leiva, Maria Rosa Pugliese (2001), La justicia, en: Nueva Historia de la Nación Argentina, Academia Nacional de la Historia Argentina, T. VIII, Buenos Aires, 445-470

Abasolo, Ezequiel (2008), La imagen del juez, en: Tau Anzoátegui (2008) 11-12, ver textos seleccionados $13-26$

Anastasi, Leónidas (1928), Nota al voto de Helguera, en: Revista Jurisprudencia Argentina, II, 601 (21-5-28)

Anastasi, Leónidas (1931), Prólogo Repertorio Revista Jurisprudencia Argentina, $5-7$

Anastasi, Leónidas (1938a), Prólogo Repertorio Revista La Ley

Anastasi, Leónidas (1938b), Revista La Ley, T. 7, Sección revista de revistas, 12

Anastasi, Leónidas (1938c), El juez argentino puede atenuar los rigores de una cláusula penal, en: Revista La Ley. T. 9, Sección Doctrina extranjera, 26

Battaglia (1929), L'interpretazione giuridica nella moderna letterattura francese, en: Rivista Internazionale di Filosofia del diritto, IX, separata

Belloc, Hillaire (1937), The crisis of our civilization, London

Benda, Julien (1934), L'Avenir de l'esprit europeen, Paris, Instituto de Cooperación intelectual sobre el espíritu europeo y sobre la crisis

Bibiloni, Juan Antonio (1929), Anteproyecto de Reformas al Código Civil Argentino, Buenos Aires, Ed. Abeledo

Bidart Campos, Germán J. (1982), La Corte Suprema, Buenos Aires, Allende y Brea

Bielsa, Rafael (1964-1966), Derecho Administrativo, T. II, Buenos Aires, Ed. Depalma

Bierdiaeff, Nikolá A. (1931), Un nouveau moyen age, Paris

Bовbio, NoRberto (1938), L'analogia nella logica del diritto, Torino

Воввіо, Norberto (1941), Persona e società nella filosofia dell'esistenza, separata de Archivio di Filosofia XI, f. 4, Roma

Bовbio, Norberto (1993), Perfil ideológico del siglo XX en Italia, México, F.C.E.

Bonnard, A. (1939), Le droit et l'Etat dans la doctrine national-socialiste, $2^{\circ}$. ed., Paris

Bremond, Henri (1927), L'inquiétude religieuse, Paris

Bunge, Carlos Octavio (1907), Nuestra Patria, Buenos Aires

Bunge, Carlos Octavio (1916), El derecho, ensayo de una teoría integral, $4^{\circ}$ ed., Buenos Aires

ButLer, N. M. (1933), La crisi della società contemporanea, Bari

Cabral Texo, Jorge (1919), Fuentes nacionales del Código argentino, Buenos Aires

Calamandrei, Piero (1939), La relativitá del concetto di azione, en: Scritti giuridice in onore di Santi Romano, Padova, Separata

Calamandrei, Piero (1943a), Prefacio a la obra de A. C. Jemolo, Il nostro tempo e il diritto, en: Archivio giuridico, IV

Calamandrei, Piero (1943b), La certezza del diritto e la responsabilitá della doctrina, en: Revista Diritto Comerciale, I, 341 
Capograssi, Giuseppe (1921), Riflessioni sulla autorità e la sua crisi, Lanciano

Capograssi, Giuseppe (1932), Studi sull'esperienza giuridica, Roma

Capograssi, Giuseppe (1941a), Il significato dello Stato contemporaneo, separata del volumen dedicado a L'esperienza pratica e le sue forme fondamentali, Milano

Capograssi, Giuseppe (1943), Il problema della scienza del diritto, Roma, VIII

Carnelutti, Francesco (1930), La crisi della legge, en: Rivista di Diritto Pubblico I, 424 y ss.

Carnelutti, Francesco (1937), Discorsi intorno al diritto, Padova

Carnelutti, Francesco (1940), Teoria generale del diritto, Roma

Carnelutti, Francesco (1943), Uno scritto, en: Rivista di Diritto Civile, XX, 81

Cattaneo, Carlo (1892), Logica, Firenze

Colin y Capitant (1930), Cours élémentaire de droit civil, 8 vol. En castellano Ed. $1941 / 51$

Colmo, Alfredo (1917), Técnica legislativa del Código Civil Argentino, Buenos Aires

Colmo, Alfredo (1925) Política cultural en los países Latinoamericanos, Buenos Aires

Colmo, Alfredo (1927), Técnica legislativa del código civil argentino, $2^{\mathrm{a}}$ ed., Buenos Aires

Colmo, Alfredo (1928), De la obligaciones en general, Buenos Aires

Colmo, Alfredo (1931), La justicia, Buenos Aires

Cossio, Carlos (1939), La plenitud del ordenamiento jurídico, Buenos Aires, Ed. Losada

Cossio, Carlos (1941), La valoración jurídica y la ciencia del derecho, Buenos Aires Chesterton, Gilbert (1933), Le avventure d'un uomo vivo, Milano-Roma

De Diego, Clemente (1925), La jurisprudencia como fuente del derecho, en: Revista de Derecho Privado No 12,70

De Diego, Clemente (1932), Prologo a la obra de Del Vecchio Los principios generales del derecho, Roma

Delaisi, Francis (1925), Les contradictions du monde moderne, Paris

De Stefani, Alberto (1941), Confidente e convinzioni, en: Rivista italiana di Scienze economiche, 895 y ss.

Del Vecchio, Giorgio (1933), La crisi della scienza del diritto, en: Archivio Giuridico, IV Serie, Vol. XXVII, separata, Módena

Del Vecchio, Giorgio (1935), Crisis del Derecho y Crisis del Estado, Madrid

Del Vecchio, Giongio (1936), Lezioni di filosofia del diritto, 3. ed., Roma

Del Vecchio, Giorgio (1938), Il problema delle fonti del diritto positivo, 2. ed., Roma

Del Vecchio, Giorgio (1948), Los principios generales del derecho, Barcelona

Dessauer, Friedrich (1927), Filosofia della tecnica, original en alemán, traducción en italiano, Brescia

Diario de Sesiones Congreso Nacional Argentino, Cámara de Diputados, T. 1, 1910, Exposiciones de los diputados Montes de Oca y Gonnet 
Diaz Couselo, José Maria (1986), La historia jurídica y el problema de las fuentes del derecho, en: Revista de Historia del Derecho, Buenos Aires, 14, 95-122

Diaz Couselo, José Maria (2007), El Código Civil y su reforma, en: Tau AnzoÁtegui (2007), T 1, 223-227, ver textos seleccionados 229-270

Dualde, Joaquin (1933), Una revolución en la lógica del Derecho, Barcelona, Ed. Bosch

Duguit, Leon (1901), L'Etat, le droit objectif et le loi positive, París

Duguit, Leon (1905), Las transformaciones generales del Derecho Privado, Madrid

Ferrara, F. (1911), Potere del legislatore e funzione del giudice, en: Rivista di diritto Civile

GÉNy, FrançoIs (1899), Méthode d'interprétation et sources en droit privé positif, Paris. En español, 1902

Giannini, M.S. (1939), L'interpretazione del atto amministrativo e la teoria giuridica generale dell'interpretazione, Milano

Giner De Los Rios, Francisco (1916), Principios de Derecho Natural, Madrid

Grossi, Paolo (1998), Assolutismo giuridico e diritto Privato, Milano, Ed. Giuffré

Grossi, Paolo (2006), Santi Romano: un messaggio di ripensare nella odierna crisi delle fonti, Lectio Doctoralis, en: Società, diritto, Stato. Un recupero per il diritto, Milano

Grossi, Paolo (2011a), Il novecento giuridico. Secolo Lungo, Roma, Ed. Laterza, en español El novecientos Jurídico: un siglo posmoderno, Madrid, Ed. Marcial Pons

Grossi, Paolo (2011b), L'Europa del diritto, Roma

Guenon, René (1927), La crise du monde moderne, Paris

Hauriou, Maurice (1923), Précis de droit constitutionnel, París

Hespanha, Antonio Manuel (2002), Cultura jurídica Europea. Síntesis de un milenio, Madrid, Ed. Tecnos

Huizinga, Johan (1938), La crisi della civilta, Traducción italiana, 2 ed., Torino

Instituto de Altos Estudios de la Universidad de La Plata, 2 v. Consolidación y Estudios críticos, 1930 y 1935

Jemolo, Antonio C. (1932), Il nostro tempo e il diritto, en: Archivio giuridico, IV Serie, Vol. XXIII, 129-170

Josserand, Luis (1930), Cours de droit civil positif français

Juicios críticos sobre el proyecto de código civil argentino (1920), de los doctores Manuel R. Garcia, José F. López, Manuel d. Sáez, Juan B. Alberdi, Dalmacio Vélez Sarsfield, Vicente F. Lopez, Alfredo lahitte y Victorino de la Plaza, Buenos Aires

Justo, Alberto M. (1937), La actitud del juez frente a la transformación del derecho, en: La Ley 9, Sección doctrina

Justo, Alberto M. (1963), Anastasi en la senda Jurisprudencial, en: Revista Colegio de Abogados de Abogados de La Plata, V., 10, 19 y ss.

Keyserling, Hermann (1935), La rivoluzione mondiale e la responsabilità dello spirito, traducción italiana, Milano

Kuntz, Werner (1926), Vor den Toren der neuen Zeit, Leipzig 
Lacerda, Paulo de (1936), Manual do Codigo Civil brasileiro, Direito das Obrigacoes, Rio de Janeiro

Lafaille, Héctor (1925), Curso de Obligaciones

LAGARDE, G. DE (1934), La naissance de l'esprit laique au decline du moyen âge, II, Marsile de Padove ou le premier theoricien de l'etat laique, Saint-Paul-TroisChateaux

LAMbert, JaCQues (1938), La publicización del contrato y la delegación de poderes, en: Revista La Ley, T. 12, sección doctrina, 82

Linares Quintana (1939), La legislación de emergencia en el derecho argentino y comparado, en: Revista La Ley T. 30, sección doctrina, 907 y ss.

Lopez De Oñate, Flavio (1939), Studi filosofici sulla Scienza del diritto, en: Annali della R. Universitá di Macerata, XII-XIII, Ed. Separata

Lopez De Oñate, Flavio (1943), La certezza del diritto. Ristampa postuma, con prefacio de Giuseppe Capograssi, Roma. En español La Certeza del Derecho, Buenos Aires, Ed. Jurídicas Europa-América

Losano, Mario (1997) a cura di, Storia contemporanea del diritto e Sociología Storica, Franco Angeli, Milano

Losano, Mario (2011), La geopolitica del novecento, Mondadori, Milano

Martinez Paz, Enrique (1934), Introducción al estudio del Derecho Civil Comparado, Córdoba

Martinez Paz, Enrique (1932), Sistema de Filosofía del Derecho, Buenos Aires, El Ateneo

Martire, Eduardo (2005), Las Audiencias y la Administración de justicia en las Indias, Madrid, Univ. Autónoma de Madrid

Morello, Mario (1984), en: Diario de la Revista La Ley, del 22-8-84

Morin, G. (1927), La loi et le contrat. La decadence de leur souveraineté, Paris

Mossa, Lorenzo (1930), Modernismo giuridico e diritto privato, en: Archivio di studi corporativi, 250-282

Ortega Y Gasset, José (1929), La rebelión de las masas, ver también en: Obras Completas, Madrid, Barcelona

Palacios, Alfredo L. (1920), El nuevo derecho, Buenos Aires, Lajouane y Cía

Pareyson, Luiggi (1940), La filosofía dell'esistenza e Karl Jasper, Nápoles

Perticone, Giacomo (1942), Studi sul regime di massa, Milano

Pugliese, Maria Rosa (1994), La «idea de jurisprudencia a través de los primeros años de la Revista de jurisprudencia argentina, en: Revista de Historia del Derecho 22, 241-277

Pugliese, Maria Rosa (1999), La idea de justicia en Carlos Octavio Bunge, en: Revista de Historia del Derecho 27, 261-324

Pugliese, Maria Rosa (2000), La administración de justicia, 1810-1914, Nueva Historia Argentina, T. V, Buenos Aires, Ed. Planeta, 405-438

Pugliese, Maria Rosa (2006), Juridical Journals in Argentina (20th Century). Cultural and didactic views of the doyennes still in publication, en: Stolleis, Michael, Thomas Simon (eds.), Juristische Zeitschriften in Europa, Frankurt am Main, Ed. Vittorio Klosterman, 491-532 
Pugliese, Maria Rosa (2007), La Jurisprudencia de los Tribunales, en: Tau ANZoÁtegui (2007) 273-278, ver textos seleccionados 279-341

Pugliese, Maria Rosa (2008), Las revistas jurídicas, en: Tau Anzoátegui (2008) 29-35, ver textos seleccionados 39-72

Pugliese, Maria Rosa (2010), La certeza del derecho en la Concepción de Flavio López de Oñate, en: Revista Chilena de Historia del Derecho 22, 1265-1278

Radbruch, Gustav (1931), Du droit individualiste au droit social, en: Archives de Philosophie du Droit 3,4, 387-398

Risolia, Marco Aurelio (1958), Soberanía y crisis del contrato en nuestra legislación civil, Buenos Aires

Rivarola, Rodolfo (1910), Derecho Penal Argentino, Parte general, Buenos Aires

Robbins, L. (1935), La grande crisi, traducción italiana, Torino

Romano, Santi (1918), L'ordinamento giuridico, Pisa

Ruggiero, R. (1927), I dogmi del diritto privato e la loro revisione, en: Archivio giuridico, IV serie, Vol. XIII, 133-153

SAleEby (1908), La preoccupazione, ossia, la malattia del secolo, traducción italiana, Bari

Salvat, Raymundo (1938a), Tratado de Derechos Reales, T. I, 347 y ss., Buenos Aires

Salvat, Raymundo (1938b), Teoría del abuso del derecho, en: Revista La Ley, T. VI, sección doctrina, 51 y ss.

SCHeler, Max (1936), La crisi dei valori, traducción italiana, Bari

Segovia, Lisandro (1874), El Código Civil con su explicación y crítica, Buenos Aires

Scholten, P. (1936), L'interpretation de la loi et la justice, en: Annales de l'Institut de Droit Comparé de l'Université de Paris

Sorel, Georges (1941), I cattolici contro Bergson, en: L'Europa sotto la tormenta, Milano

Spengler, Oswald (1919), Der Untergang des Abendlandes, Vol. I, 4 ed., München

Strowski, Fortunat (1931), L'homme moderne, Paris

Tau Anzoátegui (1974), Víctor, Los juristas Argentinos de la generación de 1910, en: Revista de Historia del Derecho, 2, 225-283

Tau Anzoátegui, V. (1997), Las ideas jurídicas en la Argentina, 3ra Ed. Abeledo Perrot

Tau Anzoátegui, Víctor (coord.) (2007-2008), Antología del pensamiento juridico Argentino (1901-1945), 1-2, Buenos Aires, Ed. Instituto de Investigaciones de Historia del Derecho

TAu Anzoátegui (2011), La jurisprudencia civil en la cultura jurídica argentina (s. XIX-XX), en: Quaderni Fiorentini 40, 53-110

Tilgher, Adriano (1932), Filosofi e moralisti del Novecento, Roma

Tobal, Gastón Federico (1936), El régimen de familia en el proyecto de reforma, en: Revista La Ley, T. 1, Sección doctrina, 52 y ss

Tobal, Gastón Federico (1941), La misión y aporte de nuestra Jurisprudencia, Buenos Aires

Valéry, Paul (1931), Regards sur le monde actuel, Paris 
Vierkant (1932), La actitud íntima del hombre actual frente al derecho, en: Revista de Occidente CXIII, noviembre 1932

Veit, O. (1937), La tragedia dell'età tecnica, trad. italiana, Milan

Zimmermann, Eduardo (1995), Los liberales reformistas, la cuestión social en la Argentina (1890-1916), Buenos Aires 


\section{Crisis del derecho privado y legislación especial en Francia y en Argentina}

\section{Europa - Argentina: trasplante de prácticas y de discursos}

En 1912 el célebre profesor francés Léon Duguit visitaba Buenos Aires y pronunciaba allí una serie de conferencias de materia jurídica. El bordelés viajó a la América Austral invitado por la universidad de Buenos Aires en sustitución de otro profesor, Raymond Saleilles, que había declinado la invitación por razones de salud. ${ }^{\mathbf{1}}$ En todo caso las conferencias causaron sensación en la Argentina. Acudieron, entre otros, Ernesto Quesada, Juan Agustín García, Juan José Díaz Arana, José León Suárez, Alejandro Ruzo, y Alfredo Palacios. ${ }^{2}$ Las conferencias fueron publicadas en forma de libro en castellano en 1912 con el título Las Transformaciones generales del derecho privado desde el código Napoleón. ${ }^{3} \mathrm{Al}$ año siguiente se publicó en francés: Les transformations générales $d u$ droit privé. En la introducción afirmaba lo siguiente: «J'ai essayé de montrer que l'évolution juridique est, dans ses lignes générales, identique dans tous les pays parvenus à peu près au même degré de civilisation, et qu'elle se caractérise par la substitution constante et progressive d'un système juridique d'ordre réaliste et socialiste à un système d'ordre métaphysique et individualiste.» ${ }^{4}$

* Esta es la versión escrita de la ponencia presentada en Buenos Aires en julio de 2012, reelaborada durante una estancia en el Max-Planck-Institut für europäische Rechtsgeschichte de Frankfurt am Main y en el Iberoamerikanisches Institut de Berlín financiada por la AGAUR del Gobierno de Cataluña. Agradezco los comentarios, críticas y aportaciones de los participantes en el encuentro de Buenos Aires y del Seminario Latino del Instituto Max Planck. Agradezco la valiosa ayuda del personal del Max-Planck-Institut y del Instituto iberoamericano y a Francisco Andrés Santos y a Maria Vila por la lectura y las aportaciones.

1 Así lo explica Thaller (1914) 36.

2 Así lo explica Zimmermann (2013) 89. Lo extrae del periódico La Prensa, 27 de agosto de 1911: 7.

3 Duguit (1912).

4 Duguit (1913) 1. 
El autor francés señalaba un fenómeno importante en la época en la que escribía y que nos interesa también a los que escribimos sobre las transformaciones del derecho privado: el proceso de socialización del derecho privado en Europa y su influencia en América Latina.

Duguit estába convencido de que la evolución del derecho en los países llegados a un determinado nivel de civilización, concepto no exento de eurocentrismo e incluso tintes racistas, ${ }^{5}$ seguía los mismos parámetros. El proceso de industrialización desarrollado en Europa había saltado a algunas regiones y ciudades de la América Latina y los problemas que generaban la concentración de capital y la aparición del proletariado necesitaban de soluciones similares. Por ello hubo transferencia de conocimientos, de tecnología, de modelos de organización económica y también de ideas y modelos políticos de la Europa industrializada a la América en trance de industrialización. Estas transferencias se daban en un mundo cada vez más globalizado pero que seguía teniendo su centro simbólico y político en Europa Occidental.

Las consecuencias del desarrollo industrial ponían en jaque los conceptos del liberalismo jurídico contenidos en el código Napoleón. La llamada cuestión social, definida por James Morris como el conjunto de consecuencias sociales, laborales e ideológicas derivadas de la industrialización y la urbanización, ${ }^{6}$ ponía en jaque no solo las normas de derecho privado sino todo un modelo social y político.

Los accidentes de trabajo, la miseria de las clases proletarias, la desprotección de ancianos y niños considerados como inevitables y connaturales al desarrollo industrial alimentaron a mediados del XIX discursos y estrategias de partidos y sindicatos en toda Europa. La amenaza socialista provocó que juristas, parlamentarios, empresarios y la Iglesia católica reaccionasen a finales de ese siglo ante fenómenos que no eran nuevos aunque pero que en ese momento amenazaban la reproducción del sistema capitalista. Tuvo en ello que ver el desarrollo del socialismo. También la estadística y los medios de comunicación de masas permitieron que estos fenómenos fuesen codificados, explicados, difundidos y objeto de discusión.

5 Raymond Saleilles hablaba por ejemplo de «Humanité civilisée» para referirse a la humanidad de los países occidentales. Vid. Aragoneses (2009).

6 Morris (1966) 78. Citado por Schjolden (2009) 92. 
En América fue Argentina el primer país que experimentó el modelo de industrialización importado de Europa. Por ello a inicios del siglo XX se reprodujeron allí los debates que se daban o se habían dado en Francia, en Alemania y en España a finales del siglo XIX y el desarrollo industrial argentino estuvo también acompañado por un proceso de producción y difusión de ideas y proyectos políticos y legislativos similar al que se había dado poco antes en el viejo continente.

En sus conferencias en Buenos Aires Duguit señalaba que la evolución del derecho en la Argentina seguía los mismos parámetros que la que se estaba produciendo en Europa. Esta afirmación, leída desde la distancia, resulta cierta a medias. Efectivamente el derecho argentino también sufre un proceso de modernización / socialización a comienzos del siglo XIX similar al que había comenzado unos años antes en Europa. El lenguaje jurídico argentino era muy parecido al español o al francés. Hubo trasplante de obras, de revistas, de discursos en definitiva que contenían un arsenal terminológico que en Europa había servido para llevar a cabo una aproximación más socializante y realista al derecho. Podemos encontrar una continuidad terminológica entre los discursos europeo y argentino, pero esta no siempre implica una continuidad semántica. Los términos eran los mismos que los de Europa pero, al ser trasplantados a la Argentina, eran leídos de forma diferente porque diferente era el contexto social y cultural. En mi opinión se importó de Europa un léxico pero se le dio un significado y una función diferentes de las que tenía en Europa. Los términos trabajo, socialismo, sindicatos o Nación tenían significados distintos en la vieja Europa y en la Argentina. Ni el papel del Estado era el mismo, ni lo era la función de la religión o los sindicatos.

Tampoco es comparable la composición de la sociedad, más heterogénea en América, ni la función jugada por el nacionalismo en uno y otro lado del Atlántico. En América, también en Argentina, encontramos naciones en construcción en los discursos políticos pero también en la práctica política: la Nación argentina se construye con el componente criollo, el indígena y una inmigración europea que se dispara en esos años. La idea de Nación, la argentinidad, juega un papel cohesionador de la sociedad importantísimo que obliga a definir lo argentino mediante su diferenciación con lo europeo. Y lo mismo podemos decir de la función jugada por la Iglesia y la religión en uno y otro hemisferio. Pese a la pluralidad religiosa americana, la referencia 
a la religión juega también una función de cohesión social que había perdido en Europa. ${ }^{7}$

Todo ello nos obliga a replantear determinados conceptos, como el de trasplante o recepción legislativa, a la hora de analizar el uso de doctrinas y textos legislativos en los debates académicos o parlamentarios en Argentina. Es conveniente cuestionar la idea de que hubo una simple recepción o adaptación de ideas mediante la importación y adaptación de textos legislativos o doctrinales sobre la cuestión social. En mi opinión hubo una construcción de nuevas ideas, de nuevas instituciones, si bien en este proceso se utilizaron textos portadores de un léxico europeo que en Europa había construido realidades bien diferentes.

Debemos tener también en cuenta que detrás de toda propuesta de cambio jurídico, sea doctrinal, jurisprudencial o legislativa, hay un modelo de sociedad. En los años finales del siglo XIX y comienzos del XX convivían en Europa proyectos políticos liberales, liberal-corporativos y socialistas. También se trasplantaron esos proyectos en Argentina. Pero considero que los proyectos homónimos de organización social y política no son idénticos a ambos lados del Atlántico y, además, no se expresan mediante el mismo tipo de propuestas normativas.

En el presente texto trato la respuesta jurídica a los accidentes de trabajo como un fenómeno de trasplante doctrinal y legislativo entre Europa y la Argentina. Se trata en un primer momento de un cambio en la doctrina civilista en Francia y después en Argentina que intenta superar las limitaciones de la responsabilidad subjetiva. Ante su insuficiencia y por la presión de organizaciones políticas y científicas se promulgaron en Europa de leyes especiales que también fueron trasplantadas en Argentina en muchos casos literalmente. Intento mostrar que esta identidad terminológica de leyes, expresiones o instituciones europeas y argentinas muchas veces no conlleva identidad semántica, ya que en el país receptor, Argentina, se usa el léxico jurídico o político europeo en muchas ocasiones de manera distinta, para sostener proyectos diferentes al que originase ese «Thesaurus» en Europa. En definitiva: tanto en la discusión doctrinal como en el debate sobre la ley de accidentes de trabajo hubo en Argentina trasplante de terminología y de léxico. Esta importación de terminología ya supone, según Watson, una

7 Oddone (1984). 
influencia del derecho europeo sobre el derecho argentino que merece nuestra atención ${ }^{\mathbf{8}}$ aunque las circunstancias y los efectos de esa importación fueran distintos en uno y otro continente porque en esos años, no solamente se generaba un derecho social en la Argentina sino que, además, se estaba forjando la identidad nacional del joven país.

Francia 1896: los juristas y los accidentes de trabajo

A finales del siglo XIX los accidentes de trabajo se habían convertido en un fenómeno frecuente y aceptado socialmente como inherente a un determinado modelo de desarrollo industrial. No sabemos en qué momento se convirtieron en una amenaza para la reproducción de la fuerza de trabajo y en una realidad que alimentaba el caballo de batalla del socialismo. Seguramente a medida que se intensificaba la industrialización había más accidentes pero el desarrollo de los intercambios de información con los medios de masas y el desarrollo de disciplinas como la estadística ayudaron a que los accidentes se situasen en el centro de los discursos políticos y jurídicos.

En todo caso es a finales del siglo antepasado cuando la cuestión de los accidentes de trabajo pasó a ocupar a parlamentarios, juristas y sociedades científicas en Francia. ${ }^{9}$

En el derecho legislado este fenómeno no estaba regulado específicamente. Se aplicaban las reglas del código civil, auténtica constitución económica de Francia, sobre la responsabilidad extracontractual. El artículo 1382 del código civil francés rezaba así «Tout fait quelconque de l'homme, qui cause à autrui un dommage, oblige celui par la faute duquel il est arrivé, à le réparer». Este breve precepto expresaba una de las más importantes "reglas del juego de la paz burguesa": ${ }^{10}$ la responsabilidad subjetiva o responsabilidad basada en la culpa: solamente se respondía por el daño causado por dolo o negligencia. Sin estos elementos no había obligación de compensar el daño. Esta construcción era útil como principio general en una sociedad preindustrial. De hecho había sido un instrumento de construcción del mercado burgués. Pero en la Francia industrializada de finales del siglo XIX esta

8 Watson (1993).

9 Sobre la discusión jurídica de estas cuestiones vid. Halpérin (1996).

10 La expresión es de Arnaud (1973). 
norma chocaba con la dura realidad: la búsqueda del máximo beneficio llevaba a los empresarios a innovar y mecanizar cada vez más la producción mediante máquinas que implicaban riesgo: calderas de vapor, brazos mecánicos, rieles ... Los accidentes normalmente no se producían ni por culpa del patrón ni por culpa del trabajador o del ingeniero. En consecuencia no existía responsabilidad ni obligación de indemnizar al trabajador herido o a los sucesores del fallecido en caso de muerte.

Este estado de cosas tenía graves consecuencias humanas. Pero llegó un momento en que también comenzó a ser una amenaza para el sistema capitalista, al poner en riesgo la reproducción de la fuerza de trabajo y disminuir la masa que consumía los bienes de la industria. En caso de muerte o de invalidez permanente el obrero o/y su familia eran expulsados del sistema productivo: no tenían recursos para consumir los productos de la industria y suponían una carga financiera al pasar a depender de las organizaciones de beneficencia. No hace falta mencionar que esta situación también alimentaba el fantasma del comunismo que tanto temor provocaba en las elites.

Ante las limitaciones de la regulación del código civil para dar respuesta a este fenómeno los juristas franceses buscaron en la doctrina nacional y extranjera modos de adaptar la norma a los «tiempos modernos». Uno de los juristas que destacaron en este proceso fue el comparatista Raymond Saleilles. El profesor borgoñón es conocido por haber desarrollado la idea del «risque professionel». Para ello se basó en una célebre sentencia, la del caso Teffaine del año $1896 .{ }^{11}$ Esta sentencia muestra la importancia de la jurisprudencia a la hora de dar respuesta a los desafíos de la sociedad industrial.

En esta decisión el Tribunal debía fallar sobre el derecho de los sucesores de un obrero fallecido por las heridas que le había provocado la explosión de una caldera. El juez reconoció el derecho a una indemnización basándose no en el artículo 1382 sino en el 1384, que regulaba la responsabilidad por guarda de la cosa: «On est responsable non-seulement du dommage que l'on cause par son propre fait, mais encore de celui qui est causé par le fait des personnes dont on doit répondre, ou des choses que l'on a sous sa garde». Esta sentencia abría por tanto una vía para solucionar la falta de indemni-

11 Cassation Civile 16 juin 1896.

$124 \quad$ Alfons Aragoneses 
zación que causaban los accidentes de trabajo. También es cierto que esta sentencia fue apelada y que la instancia judicial superior la anuló. Pero sirvió de todos modos para que la doctrina plantease propuestas.

Saleilles utilizó la interpretación dada por la jurisprudencia a los artículos 1382 y 1384 en el caso Teffaine para desarrollar su teoría del riesgo profesional plasmada en un librito de $1897^{12}$ y en un artículo de 1898 en La Reforme sociale ${ }^{\mathbf{1 3}}$ órgano de la Société d'Economie sociale, la organización creada por el reformista social Frédéric Le Play. Saleilles intenta objetivar algo difícilmente objetivable: el riesgo que, al entrar a trabajar en una fábrica o en un ferrocarril, el obrero asumía para obtener un salario y para crear un beneficio para el empresario. Saleilles utiliza el razonamiento del tribunal del caso Teffaine: la responsabilidad por la guarda de la cosa del artículo 1384 se aplicaría en caso de accidente de trabajo al ser el empresario el propietario de la maquinaria. El propietario de la fábrica poseía unas máquinas que eran peligrosas de manejar. El propietario era el que se beneficiaba de la exposición al riesgo del obrero. Por tanto el propietario debía asumir las consecuencias de este.

Esta argumentación generó una importante discusión en torno al concepto de riesgo profesional. Algunos autores se apoyaban en Saleilles para afirmar que esta idea de riesgo profesional venía determinada por el código. Otros como Glasson, Labbé, Lyon-Caen, Esmein et Planiol consideraban que la responsabilidad se derivaba de las obligaciones contenidas en el contrato. ${ }^{14}$ Pero todos coincidían en la necesidad de superar los estrechos márgenes que dejaba el artículo 1382 del código civil.

Otra alternativa de estos privatistas «fin de siècle» era la de una reforma limitada de los códigos civiles. La obra del belga Charles Sainctelette, recibida en Francia y admirada por Raymond Saleilles, defendía también una solución dentro del código civil, aunque en este caso a través de una pequeña reforma que incluyes una frase estableciendo que «Quiconque loue les services d'un domestique ou d'un ouvrier s'oblige à le tenir indemne des suites de tout accident de travail qu'il ne justifie pas provenir d'une cause étrangère

12 Saleilles (1897).

13 Saleilles (1898).

14 Saleilles (1897) 13. 
qui ne peut lui être imputée.» ${ }^{15}$ Con esta frase se solucionaría el problema de la indefensión del obrero accidentado.

Las teorías de estos «juristes néoclassiques», ${ }^{\mathbf{1 6}}$ por usar la expresión de Jamin y Verkindt, defendían la reforma del derecho mediante la adaptación jurisprudencial y doctrinal de los preceptos del código. Se trataba de ir «audelà du code civil mais par le code civil» por citar una expresión cara a Saleilles: más allá del dictado literal del código pero siempre respetando al código. Detrás de esta propuesta hay un modelo de sociedad, un modelo que llamaría «modernista». ${ }^{17}$ Defendía el statu quo liberal mediante la adaptación de sus viejos postulados y la posición privilegiada de la élite de los juristas, evitando así la intervención del Estado, que debía limitarse a conservar el orden público. Saleilles reconoce la existencia de una división entre patrones y obreros, rechaza la intervención del Estado para paliarla y defiende una adaptación del credo liberal a la luz de la doctrina católica y la fe en la capacidad autorreguladora de la sociedad.

En este modelo de sociedad y de derecho, el juez tenía «une mission sociale très haute, qui consiste à rechercher (...) dans l'objectivité des réalités économiques et sociales, les créations juridiques susceptibles de s'adapter à l'ensemble de la loi». ${ }^{\mathbf{1 8}}$ El juez en que piensa Saleilles tenía que jugar una importante función social al permitir el desarrollo de un sistema que frenaba el desarrollo del socialismo jurídico, ${ }^{\mathbf{1 9}}$ y es que esta renovación de la doctrina civilista no se explica sin la amenaza del socialismo: «Demandez aux socialistes - escribe Saleilles en 1906 - demandez aux féministes, de quelle arme ils comptent se servir pour faire pénétrer sans trop de secousses leurs revendications dans notre état social: De la méthode juridique, vous répondrontils. Il y avait autrefois un socialisme révolutionnaire. Je ne veux pas dire, hélas! Qu'il n'y en ait plus. Mais il y en a plus, aujourd'hui, un socialisme juridique.» 20

15 Sainctelette (1886) 66.

16 JAMin/VERKindt (2003).

17 La expresión «jurista modernista» es de Cesarini Sforza. Grossi (2000) 163 la recupera. Vid. Aragoneses (2009).

18 Saleilles (1904) IX, 21. Citado por Herrera (2011) 336.

19 Herrera (2011) 335-343 et 366.

20 Saleilles (1911) XXII.

126 Alfons Aragoneses 
Fracaso del «derecho común» y triunfo de la «solución legislativa» en Europa

La historiografía jurídica dedicada a la evolución del pensamiento jurídico había estudiado estos importantes cambios de la doctrina en el ámbito de la responsabilidad. ${ }^{21}$ Pero muchos de estos estudios se centraban en las producciones discursivas de la doctrina privatista e ignoraban el debate que sobre los accidentes de trabajo se daba fuera de las cátedras de derecho privado. Y es que en esos «temps des trublions» se discutía la cuestión social fuera de los ámbitos de los jurisperitos y había propuestas que iban mucho más allá del código civil.

Saleilles, experto en la doctrina y en la legislación alemanas, lo sabía perfectamente. En su texto de 1896 presenta su solución «interpretativa» al problema de los accidentes de trabajo: la de los privatistas que reinterpretan los artículos del código. Pero reconoce, en una nota a pie de página, que había autores que «ont fait davantage peut-être en vue de la solution législative de la question». ${ }^{22}$ A continuación dejaba clara su opinión sobre la misma: los juristas debían «être reconnaissant à ceux, qui par un mouvement de doctrine et de jurisprudence, permettent d'aboutir à des changements d'interprétation qui équivalent à des véritables réformes pratiques en attendant l'intervention de la loi». ${ }^{23} \mathrm{Al}$ respecto escribe Saleilles que «les tâtonnements de la doctrine et les essais plus ou moins hardis de la jurisprudence ont démontré, avec une éloquence plus saisissante encore que toutes les statistiques, la nécessité de sortir de l'application et de l'interprétation traditionnelles de l'article 1382». ${ }^{24}$

¿Cuál era la solución legislativa? ¿Por qué esta referencia a la relación entre estadística y doctrina? Saleilles estaba pensando en las discusiones que tenían lugar en Francia y que tenían la mirada puesta en la legislación alemana sobre accidentes de trabajo, que abría la puerta a un nuevo modelo en el que la intervención del Estado era fundamental. Y Saleilles piensa también en el apoyo que la estadística y las ciencias sociales daban a esta solución legislativa.

21 Cazzetta (1991), Aragoneses (2009).

22 SAleilles (1897) 2, nota 2.

23 Ibidem.

24 Ibidem. 
Saleilles defiende una teoría del riesgo profesional dentro del código civil pero sabe que la tendencia en política legislativa en Europa es otra. En 1897 escribe «Je suis loin de m'opposer à ce qu'on fasse en France une loi sur la responsabilité industrielle. Il y a là un terrain nouveau qui demande une réglementation nouvelle, à condition qu'elle ne soit pas trop étroite et qu'elle ne devienne pas tyrannique.» ${ }^{25}$ Para Saleilles, «en attendant la loi, nous pouvons (...) découvrir dans le Code civil lui-même, non seulement le principe, mais aussi l'application même du risque industriel». ${ }^{\mathbf{2 6}}$ Saleilles creía que la solución legislativa se debía adoptar una vez la jurisprudencia y la doctrina hubiesen evolucionado lo suficiente: «Le progrès ne peut se faire que par l'adaptation quotidienne du droit aux faits qui se révèlent, et très peu, j'imagine, par ces procédés de condensation législative qu'on appelle des lois organiques, qui réduisent tout à de grandes lignes un peu trop simples.» ${ }^{27}$

¿Quienes defienden la «solución legislativa» tan errada según Saleilles? Juristas, economistas, empresarios e ingenieros que están en contacto con la práctica industrial y, preocupados por la cuestión social que conocen bien, demandan cambios. Pero piden reformas legislativas de profundidad. Desde los años 1880 encontramos en Francia publicaciones periódicas que se ocupan del tema, motivadas seguramente por la aprobación en Alemania y en Austria de leyes especiales sobre accidentes de trabajo y por la fuerza del movimiento socialista y también de la doctrina social de la Iglesia. Sin querer ser exhaustivo y apoyándome en un texto de Francis Hordern, ${ }^{28}$ en esos años se publica la Revue d'économie politique creada por el jurista Charles Gide. A partir de 1900 se encuentra en las hemerotecas la revista Questions pratiques de législation ouvrière. A estas dos cabeceras hay que añadir La Réforme sociale, que ahora analizaré, la Revue socialiste, creada en 1885 por el «communard» Benoît Malon o el Bulletin de l'Office du travail, aparecido en 1894.

Desde 1886 se publica la Réforme Sociale, el Bulletin de la Société d'Economie sociale et des Unions de la Paix Sociale. Se trata de la sociedad de los sucesores de Frédéric Le Play, reformista inspirado por la doctrina social

25 Saleilles (1898) 637.

26 Ibidem.

27 Saleilles (1898) 638.

28 Hordern (2001) 43.

128 Alfons Aragoneses 
de la Iglesia, y que reunía a empresarios, juristas, médicos interesados por la cuestión social, especialmente la cuestión obrera. Hablan de historia del derecho, de la propiedad de los obreros, del alcoholismo y también de los accidentes de trabajo. Y escriben autores que defienden una solución legislativa al problema, como Emile Cheysson, ingeniero y empresario y uno de los intelectuales más implicados en el tema de los accidentes de trabajo en la época.

Entre las publicaciones dedicadas a la cuestión social nos interesa una que se ocupa específicamente de los accidentes de trabajo. En 1889 aparecen publicadas las actas del «Congrès international des accidents de travail», ${ }^{29}$ que seguirían publicándose en los años siguientes como Bulletin du Comité Permanent des Congrès internationaux des Assurances sociales / Congrès international des Accidents du Travail et des Assurances social. El congreso había sido organizado por el Ministerio francés de Comercio, Industria y Colonias. Esto prueba el interés del Estado francés en abordar el tema de los accidentes de trabajo. Las actas del congreso están divididas en tres partes: una sección técnica, una sección de estadística y administración y una sección de economía y legislación. El derecho, por tanto, es solamente una de las formas de abordar la cuestión, no la única y se entiende como equivalente de legislación, más que de doctrina.

En esta publicación encontramos de nuevo el nombre del ingeniero Emile Cheysson. También participan en esta organización juristas, empresarios, médicos o parlamentarios como Numa Droz, diputado en la asamblea nacional suiza. Hay participantes franceses, suizos y belgas. Se trata por tanto de un foro multidisciplinar y con una fuerte apuesta comparatista que se va a apoyar decididamente en las ciencias sociales en general y en particular en la estadística, que vamos a encontrar mencionada con mucha frecuencia. No encontramos, en cambio, profesores de derecho civil de las facultades en las que se discute la «solución interpretativa». Esto nos da pistas sobre cómo la civilística quedaba al margen de las corrientes legislativas de la época, tendentes al desarrollo de normas fuera del código.

Si los profesores de derecho civil ignoran las propuestas de «solución legislativa», los partidarios de esta última conocen y discuten lo que llaman «el régimen de derecho común». Es decir: el sistema de responsabilidad 
subjetiva derivado de los códigos civiles. Afirma el parlamentario Numa Droz que «L'Etat ne peut donc s'en rapporter au seul intérêt individuel du soin de prévenir les accidents, ni s'en tenir au droit commun pour la réparation du dommage causé, mais il doit prescrire lui-même des mesures préservatrices et pourvoir à ce que les victimes d'accidents puissent obtenir comme une chose due et non comme une aumône volontaire, les secours qui leur sont indispensables.» ${ }^{\mathbf{3 0}} \mathrm{El}$ sistema de derecho común, además, imponía una gran carga económica y procesal al obrero accidentado o a su familia pero también al empresario, obligado en caso de ser condenado a pagar unas indemnizaciones ruinosas. Por todo ello se proponía normas especiales que regulasen la materia fuera del sistema de derecho común de la responsabilidad.

En estas asociaciones se discute sobre la cuestión social y también se hacen propuestas de reformas legislativas. Para ellas la solución está en la legislación especial. Se discute solamente el tipo de normativa a aprobar. Sobre estas discusiones y proyectos planea la sombra del sistema alemán de seguros: continuamente se compara, se critica o se alaba la ley alemana de accidentes de trabajo de 6 de julio de 1884, la primera de la historia y que marcó las legislaciones de todos los países europeos y, por tanto, las americanas también. La ley alemana puso delante del espejo todos los sistemas jurídicos en esta materia.

Lo describía muy bien Cheysson cuando escribía que la ley del Imperio alemán sobre accidentes de trabajo provocaba «une fascination (...) sur tous les pays en mal de réformes ouvrières et même sur les esprits les plus libéraux». ${ }^{31}$ Cheysson admira la solución alemana aunque no comparte el socialismo de Estado que según él inspiraba la legislación del II Imperio. Y es que la opción alemana por el Estado como gestor de un sistema de seguros sociales era lo que más reticencias levantaba en las elites francesas o suizas que rechazaban una excesiva intervención del Estado y apostaban más por soluciones de corporativismo suave en las que pesase más la organización dentro de las empresas con alguna intervención de los obreros pero dejando la iniciativa a los empresarios.

30 Droz (1889) 10.

31 Cheysson (1889) 452.

130 Alfons Aragoneses 
En Alemania la ley de 1884 había impuesto un sistema de seguro obligatorio organizado y controlado por el Estado. Este sistema, imitado por Austria en 1887, permitía que las empresas tuviesen las mismas ventajas competitivas ya que el Estado establecía el mismo sistema de control y desarrollo de estos seguros. Este sistema restringía la libertad del empresario pero garantizaba que los obreros de todas las industrias afectadas estuviesen protegidos.

Como había pasado con el código civil alemán, también en el caso de los accidentes de trabajo la obra del legislador alemán determinaba las discusiones y la producción normativa en la mayoría de países de la Europa continental. Las discusiones del Congreso internacional de accidentes de trabajo o de La Réforme sociale no quedaban al margen de la creación reciente del primer sistema de seguros sociales en Europa continental.

El reformismo alemán y las sociedades científicas provocaron la emergencia de un debate en Francia sobre la ley de accidentes de trabajo. Tarbouriech, civilista autor de dos obras sobre la materia ${ }^{32}$ citadas de refilón por Saleilles, ${ }^{33}$ explicaba los argumentos utilizados en los años ochenta por algunos diputados, que basaban su rechazo en la defensa de los principios de igualdad de la revolución francesa. No se podían aprobar leyes especiales porque iban en contra del principio de igualdad y suponían un retorno al pasado, ${ }^{34}$ lo que muestra la importancia de los aspectos culturales en estos procesos.

Los autores que participan en la Réforme sociale o en el Bulletin des congrès des accidents de travail hacen con frecuencia referencias, expresas o tácitas, a la doctrina social de la Iglesia. Se habla de armonía, de cooperación entre obreros y patronos. Emile Cheysson, en un texto de 1889, hace referencias a la idea de la solidaridad social necesaria para frenar el drama de los accidentes, llegando a escribir que el problema no existía en el pasado gremial, al ocuparse la comunidad de los accidentados y sus familias. ${ }^{35}$ Este lenguaje conecta directamente con el de la Encíclica Rerum Novarum, que marcó el camino de la doctrina social de la Iglesia y también el de las teorías corporativistas del siglo $\mathrm{XX}$.

32 Tarbouriech (1896); Tarbouriech (1889) 97-98.

33 Saleilles (1897) 2, nota 2.

34 Tarbouriech (1896) 85 y ss.

35 Cheysson (1889) 451 y ss. 
Cheysson alababa al legislador francés que en los ochenta, cuando discutía una de las propuestas de regulación de los accidentes de trabajo, había resistido " à l'attraction toute-puissante de la loi allemande et (...) reculé devant l'assurance obligatoire $»{ }^{36}$ La alternativa francesa a la ley alemana era en esos años la de un seguro voluntario, organizado por las asociaciones de empresarios, ${ }^{37}$ lo que dicho por un ingeniero representante de estos muestra también que el proyecto organicista estaba presente entre la clase empresarial. Se apostaba por la autorregulación con una mínima intervención del Estado.

Además de estas sociedades en esa época algunos gobiernos deciden crear órganos consultivos en materia de trabajo para facilitar el estudio y la preparación de normas. En Francia, a falta de un Ministerio de Trabajo, que todavía atemorizaba a conservadores y también a republicanos, se crea en 1891 el Conseil Supérieur du Travail, con competencias puramente consultivas. Este consejo crea el mismo año el Office du Travail, con el objetivo de realizar investigaciones y elaborar estadísticas sobre el mundo del trabajo (trabajadores por sectores, presencia femenina, menores, horas de trabajo ...) y poder así fundamentar mejor las propuestas legislativas o informes del Conseil Supérieur. La evolución de esta parcela del derecho no se entiende sin el conocimiento del mundo del trabajo que facilitan los estudios y las estadísticas de este Consejo superior, auténtico precedente del Ministerio de Trabajo francés. ${ }^{38}$ Estos organismos, además, protegían el debate de la discusión política, única forma de liberar esta parte del derecho de inercias del

No podemos ignorar que, además de estas propuestas, discusiones, estudios, etc., en Francia se extendía la práctica del seguro colectivo, «assurance collective». Los empresarios, conscientes de los problemas que suponían los accidentes de trabajo y también de la evolución no solamente de la jurisprudencia sino también de la conciencia social, comenzaron a contratar seguros de accidentes con empresas aseguradoras, en ocasiones formadas por asociaciones de empresarios, las «sociétés mutuelles». Se trata de una práctica asociativa y contractual que comienza en la zona del Sena hacia finales de los años ochenta en los sectores de la construcción y de las canteras y que se

36 Cheysson (1889) 470-471.

37 Ibidem.

38 Decouflé (1984).

132 Alfons Aragoneses 
fue extendiendo por sectores y por regiones. En 1898, año de aprobación de la ley de accidentes de trabajo las empresas francesas tenían contratados seguros por valor de veinte millones de francos. ${ }^{39}$ Un sector de la doctrina había comenzado incluso a teorizar sobre este tipo de contratos de seguros colectivos. $^{40}$

Estos empresarios fomentaban la discusión en las sociedades científicas sobre el tema de los accidentes de trabajo. La prueba la tenemos en la lista de autores e intervenciones en el Congreso internacional o en La Reforme sociale. Pero especialmente se habían avanzado en la práctica. Sin duda el interés era organizar un régimen de seguros que evitase la intervención del Estado y que solucionase la grave cuestión de los accidentes mediante la autoorganización y la autorregulación. Este proyecto de los empresarios se materializaba en la práctica de las «assurances collectives» y mediante la defensa en las sociedades científicas de proyectos de corte corporativo.

En los años ochenta del siglo XIX se habían discutido proyectos de ley en el parlamento francés sin que ninguno progresase. Como explica Tarbouriech, los proyectos de leyes especiales tuvieron una fuerte oposición por considerar que atentaban contra «les principes de 1789», es decir: la radical igualdad formal y el rechazo a regímenes especiales. ${ }^{41}$ Los intereses de clase tomaban forma cultural, en este caso apelando a la cultura jurídica francesa y al mito fundacional de $1789 .{ }^{42}$ A esta oposición basada en elementos económicos y políticos se tuvieron que enfrentar los socialistas que, como Jean Jaurès, luchaban por la aprobación de una ley especial sobre accidentes de trabajo.

Finalmente la práctica empresarial, la lluvia fina de los debates en sedes científicas y políticas y también la demanda empresarial de establecer las mismas condiciones laborales en todas las empresas allanaron el camino del proyecto de que el Parlamento sanciona como «Loi du 9 avril 1898 sur les responsabilités des accidents dont les ouvriers sont victimes dans leur travail».

La ley de 9 de abril establecía un sistema por el que el empresario era responsable de los daños en caso de accidente de trabajo del obrero. En un

39 Garmendia (1918) 38.

40 Tarbouriech (1889) 1 y ss.

41 Tarbouriech (1896) 97-98.

42 Sobre los mitos fundacionales en la historiografía en general vid. Giondano (1996). 
primer momento se aplicaba a determinados sectores industriales. La jurisprudencia, la ley y los reglamentos fueron ampliando el ámbito de aplicación. En la norma se definía el accidente de trabajo y la indemnización, que en realidad era una pensión por alimentos. La ley no obligaba al empresario a contratar un seguro, pero al ser él mismo el responsable de los daños del obrero, indirectamente se le estaba invitando a hacerlo, y más teniendo en cuenta que debía financiar la «Caisse Nationale pour la Vieillesse» mediante el pago de un impuesto sobre las patentes.

La Ley de mayo de 1899 sobre la caja nacional de seguros y especialmente la de 31 de marzo de 1905 cerraron el sistema de seguros de accidentes de trabajo. Esta última norma instauraba definitivamente la obligación del empresario de contratar un seguro de accidentes. Aunque la gestión de este seguro estuvo hasta 1946 en manos empresariales, el sistema resultante dejaba poco margen a la autoorganización empresarial.

Con estas normas se abre el camino de la legislación especial en esta materia y comienza la descodificación de las normas que regulaban el trabajo. La doctrina civilista sobre responsabilidad continuaría evolucionando pero cada vez más sectores de la actividad humana le serían expropiados y acabarían regulados por la legislación especial. Cierto es que una institución de debate doctrinal como la Société d'Études législatives, creada entre otros por Saleilles, supo reaccionar y trató en sus sesiones y estudios entre 1905 y 1908 el otro gran tema del derecho de las relaciones laborales: el contrato de trabajo. Sin duda eso ayudó a la elaboración doctrinal de esta institución y en su evolución posterior. ${ }^{43}$

Pero volvamos a los accidentes de trabajo. Con la ley se aprueba un sistema quizás menos rígido que el alemán pero que tampoco da margen para la autoorganización empresarial industrial. El Estado organiza una caja nacional que asegurará en última instancia al obrero y obliga al empresario a colaborar con ella y financiarla. El camino al corporativismo como vía intermedia entre liberalismo y socialismo no se cierra, se aplaza, como demostrará la experiencia de Vichy. ${ }^{44}$

La aprobación de esta norma tiene otra consecuencia a nivel jurídicocientífico. Se abre todo un ámbito de reflexión y discusión que acabará

43 Sobre el tema vid. Soubiran-Paillet (2004) 69-70.

44 Sobre las ideas corporativistas y su elaboración durante Vichy vid. Vielfaure (2005).

$134 \quad$ Alfons Aragoneses 
constituyendo el derecho del trabajo y de la seguridad social. Los civilistas se autoexcluyen de este debate, que se materializará en publicaciones, conferencias y también en cursos específicos. La descodificación comienza a nivel legislativo y ello afectará la aparición de nuevas disciplinas académicas.

Trasplante de doctrinas: la teoría del riesgo profesional en Argentina Las teorías y las propuestas normativas sobre los accidentes de trabajo circularon al otro lado del Atlántico casi al mismo tiempo que en Europa. La Argentina de finales del XIX «aparecía como el mejor exponente del progreso indefinido». En poco tiempo había pasado de ser un «país agrícola a ser una potencia comercial en la región». ${ }^{45}$ Pero el derecho no se había adaptado a esta nueva realidad. Seguían vigentes las normas del código aprobado en 1869 que, en materia de responsabilidad delictual, era muy similar al código francés.

La cuestión social irrumpió con fuerza en 1902 con una ola de huelgas y protestas en algunos sectores industriales. A partir de ese año se aceleran los ritmos de los debates y de las reformas. En este sentido, y como ha señalado Line Schjolden, la jurisprudencia desempeñó un papel importantísimo en la adaptación de la legislación civil y, así mediante, en el reconocimiento de nuevos derechos y en la integración en la nación de los obreros, muchos de los cuales habían nacido en Europa. ${ }^{\mathbf{4 6}}$

Un sector de la jurisprudencia argentina se adaptó a los cambios proponiendo nuevas formas de interpretar las reglas de la responsabilidad civil del código. En parte estos jueces estaban adaptando, sin decirlo, las doctrinas francesas de Saleilles y compañía.

En esos mismos años diputados socialistas y organizaciones de empresarios tomaban la iniciativa presentando proyectos de leyes especiales sobre la materia, también inspirados por normativas europeas. Al igual que en Europa, detrás de cada propuesta hay también un proyecto político. Y al igual que en Francia hay un proyecto de defensa del statu quo social que se apoya en la renovación doctrinal y jurisprudencial. A diferencia de Francia o Alemania, la sociedad argentina era mucho más plural fruto de la presencia indígena y

45 Lloyd (dir.) (1911) 1. Citado por Caterina (2008).

46 SCHJOLden (2009). 
de la inmigración europea, en parte no nacionalizada. El papel del Estado era necesariamente distinto.

Como afirma Eduardo Zimmermann, la ciencia política necesariamente debía «interrogarse sobre la naturaleza del Estado y sus mecanismos de representación». Las ciencias sociales se desarrollaban como un «intento para entender los nuevos fenómenos y como instrumento para crear unas bases sólidas para las políticas públicas orientadas a su tratamiento». ${ }^{47}$

En Argentina regía el código elaborado por Vélez Sársfield en 1869. El autor se había inspirado en el código francés, en el proyecto español de García Goyena y en el código chileno de Andrés Bello. Pese a algunas contradicciones de su redacción, la parte de derecho de obligaciones había recibido cierta influencia del Código Napoleón y de sus comentaristas, aunque este influjo fuese después exagerado, como señala Tau Anzoátegui. ${ }^{48}$ El código argentino partía del principio de la responsabilidad subjetiva, recogido en el artículo 1109: «Todo el que ejecute un hecho que por su culpa o negligencia ocasiona un daño está obligado a la reparación del perjuicio. Esta obligación es regida por las mismas disposiciones relativas a los delitos del derecho civil». Pero el mismo código civil contiene alguna norma que matiza la regla de la responsabilidad subjetiva. Si bien el artículo 900 establecía que «Los hechos que fueren ejecutados sin discernimiento, intención y libertad, no producen por sí obligación alguna». El artículo 907 rezaba «cuando por los hechos involuntarios se causare a otro algún daño en su persona y bienes, solo se responderá con la indemnización correspondiente, si con el daño se enriqueció el autor del hecho, y en tanto, en cuanto se hubiere enriquecido».

El código de comercio establecía en el artículo 156 lo siguiente: «Si en el servicio que presta el principal aconteciere al factor o dependiente algún daño o pérdida extraordinaria, será de cargo del principal la indemnización del referido daño o pérdida a juicio de arbitradores.»

Este arsenal de preceptos, con sus defectos y contradicciones, permitió una respuesta de la doctrina y la jurisprudencia al fenómeno creciente de los accidentes de trabajo. Como en Francia, también en la Argentina se da un giro en la aplicación de las reglas de la responsabilidad subjetiva en sede judicial. Hasta la aprobación de la ley de accidentes de trabajo en 1915, un

47 ZimmermanN (2005).

48 Tau Anzoátegui (2008) 264.

136 Alfons Aragoneses 
sector de la jurisprudencia, no mayoritario por cierto, desarrolla una doctrina que protege al obrero.

Schjolden habla de dos periodos en la evolución de la interpretación judicial del derecho. ${ }^{49}$ El primero sería el de la interpretación restrictiva o clásica e iría de 1900 a 1905. Se caracterizaría por la fidelidad de la mayoría de jueces al dictado literal del código. El siguiente periodo sería el de la interpretación sociológica, e iría del 1905 al 1915, año de aprobación de la ley sobre accidentes de trabajo. Hemos de tener en cuenta que nunca hubo unanimidad en la interpretación que los jueces dieron al código.

En este sentido cabe destacar la postura del célebre juez Ernesto Quesada que fue protagonista en esos años por aplicar una doctrina que recuerda bastante la del riesgo profesional. Quesada, juez y profesor, había dedicado años de su vida a visitar las universidades alemanas y francesas. De hecho a él le debemos la mejor descripción de la vida de la facultad de París disponible hoy: la obra que redacta en 1905 y publica en 1906 La facultad de derecho de París, estado actual de su enseñanza. Curiosamente de todos los profesores que conoció en París, el que más impresiona a Quesada fue Raymond Saleilles, quizás por ser este el que más compartía la germanofilia del argentino. Conviene destacar ahora las líneas que le dedica al francés. «La personalidad de Saleilles - escribe Quesada en 1905 - constituye uno de los grandes focos doctrinarios y científicos de la novísima orientación jurídica francesa. Desarrollando el impulso amplio y reformador de su suegro, el ilustre Bufnoir, ha formado escuela en el sentido de introducir la vida en el organismo secular del derecho, inspirándose en los grandes trabajos dogmático-filosóficos de la escuela germánica de Savigny y de Ihering, emancipándose de la tiranía del texto literal de la ley y de su interpretación mezquina, armonizando la letra de la legislación con la realidad de los fenómenos sociales y plegando aquélla a las necesidades de éstos. Antes de él, la jurisprudencia era el dominio de los casuistas: hoy, es el medio por el cual el factor sociológico se modela constantemente al texto anticuado de la ley codificada». ${ }^{\mathbf{5 0}}$

Otro autor que pudo influir a Quesada es Adrien Sachet, quien en 1899 publicaba su Traité théorique et pratique de la législation sur les accidents de travail. El libro, escrito con la finalidad de ayudar a jueces y abogados, tuvo

50 Quesada (1906) 217 (nota a pié). 
mucho éxito en Europa pero sobretodo en Argentina, como nos recuerda Ramacciotti. $^{51}$

Esta literatura era conocida por académicos y jueces. Y sin duda estos últimos se apoyaron en ella en su intento de adaptar el viejo código a la nueva realidad. En una célebre sentencia de 1903, por tanto durante el periodo clásico, el juez Quesada establecía lo siguiente:

«La ley comprende en su generalidad todo género de accidentes, sean o no del trabajo [...]; y comprende a los patrones, sean personas naturales o jurídicas y a todo el que trabaja por y para otro, adoptando el principio nemine officium suum debet esse damnum para todos los casos legislados especialmente en que uno trabaja por otro y para otro (arts. 1731, 1953, 1954 y 2224, C. Civil), dicho principio debe aplicarse a todos los casos análogos (art. 16, C. Civil), y lo es esencialmente el del obrero o dependiente, aunque no tenga el carácter de socio o partícipe, mandatario o depositario, puesto que trabaja por y para otro, siendo de observar que el mismo principio ha adoptado, en lo comercial, para los dependientes, socios o tripulantes (arts. 156, 414 y 1010 del C. de Comercio); por consiguiente, el patrono responde al obrero o dependiente de cualquier daño que le aviene, sin culpa, por razón del trabajo o con ocasión del trabajo, y de todo el daño que no le hubiese sucedido si no hubiera aceptado el trabajo, aunque sea por caso fortuito o fuerza mayor, pues en el silencio de la ley sobre la especialidad accidentes del trabajo, los jueces deben resolver por las analogías citadas (arts. 16 y 22, C. Civil) [...] el obrero no tiene por qué entrar a alegar si ha habido o no culpa, dolo o negligencia por parte del patrono o de su delegado: le basta alegar y probar el daño sufrido y que él le ha ocurrido en el trabajo o en ocasión del trabajo; es el patrono quien únicamente puede descargarse en el todo o en parte de su responsabilidad, alegando la compensación de la culpa del obrero que exceda de lo inherente al oficio, arte o profesión, puesto que responde hasta del caso fortuito o de fuerza mayor. ${ }^{52}$

Pocos comentarios hacen falta para entender que el fragmento citado estaba cambiando las reglas de la responsabilidad aquiliana y abría el camino al concepto de responsabilidad objetiva. En el año 1903 esta idea era muy innovadora, tanto que esta sentencia, como otras del mismo juez, no tuvo éxito en apelación: se impuso en las instancias judiciales superiores, como recuerda Schjolden, ${ }^{53}$ una interpretación más restrictiva.

Otra sentencia de Quesada de 1905, pese a ser discutida y rechazada por algunos jueces, tuvo más éxito abriendo así la fase de la interpretación socio-

51 Rammacciotti (2014) 3.

52 Citado por Levaggi (2006).

53 Schjolden (2009) 100. 
lógica. ${ }^{54}$ En esta decisión el juez Quesada, además de invertir la carga de la prueba, desligaba la responsabilidad de daño abriendo la puerta a una responsabilidad objetiva sin culpa del empresario.

En estas sentencias de Quesada encontramos una interpretación social o sociológica y un razonamiento jurídico similares al de los juristas franceses de la época. Aparece aquí también la idea de invertir la carga de la prueba. También está presente la idea de riesgo profesional como elemento objetivo. Encontramos la diferencia entre culpa y responsabilidad. Quesada no cita expresamente a Saleilles, como tampoco lo hacen los jueces franceses que beben de las ideas de la doctrina, tal como muestra Nader Hakim. ${ }^{55}$ Pero, teniendo en cuenta la similitud de sus razonamientos y la probada admiración del argentino por el francés ${ }^{56}$ podemos concluir que Quesada aplica las ideas de Saleilles y de otros franceses. Estamos, pues, ante una aplicación en Argentina de una idea que años antes había defendido un sector de la doctrina en Francia. Por tanto podemos hablar también de una solución interpretativa a la argentina. Esta solución se fue consolidando, gracias entre otros a Quesada, entre 1905 y 1915, año de aprobación de la ley. Durante ese periodo se fue generando una determinada interpretación de la normativa que, sin salir del código, facilitaba que se abriese paso la idea de la responsabilidad objetiva, no solo en la jurisprudencia, sino en el Departamento Nacional del Trabajo. ${ }^{57}$

Otros autores argentinos defendían también una solución jurisprudencial, que no legislativa, del problema de la responsabilidad derivada de los accidentes de trabajo. Destacan entre ellos Estanislao Zeballos y Juan Bialet Massé. Zeballos se va a apoyar en la jurisprudencia de Quesada y otros para defender una solución al tema de los accidentes de trabajo sin salir del código y rechazando la legislación especial. ${ }^{58}$ Bialet Massé escribió en 1904 una importante obra sobre la cuestión de los accidentes de trabajo y la responsabilidad civil en la que desarrolla su doctrina basándose fundamentalmente en el análisis del código. ${ }^{59}$

54 Sentencia del caso Olivera contra Mareyra y Othacché de 10 de mayo de 1905.

55 НАКiм (2004) 30-31.

56 Vid. supr. Nota 47.

57 SChJOLden (2009) 111 y ss.

58 Zeballos (1919).

59 Bialet Massé (1904b). 
Este argentino de origen catalán había escrito por encargo del gobierno un informe sobre las clases obreras en Argentina que pasaría a la posteridad por ser un revulsivo para los debates sobre la cuestión social. ${ }^{60}$ Se definía como socialista no doctrinario. Es decir: tenía un discurso no marxista inspirado por la doctrina social de la Iglesia. Pero lo que más destaca es su acérrima defensa del código y su rechazo a la importación o adaptación de normas especiales extranjeras. Bialet Massé pertenecía a la generación de juristas argentinos que, como decía Zimmermann, sacralizaba el código de Vélez. ${ }^{61}$ En la introducción a la monografía de Bialet Massé, el célebre discurso «A los miembros de la Comisión directiva de la Sociedad de Obreros Estibadores y de Ribera del Puerto del Rosario» utiliza un lenguaje nacionalista para rechazar la aprobación de una ley especial de accidentes de trabajo. Habla del extranjerismo que "pretende asaltar las alturas de la legislación con disposiciones exóticas, extrañas o contrarias a los principios fundamentales del Código civil» que le quita al obrero lo que le corresponde bajo pretexto de protegerlo. ${ }^{62}$ En el mismo texto se hace referencia a León XIII, a la doctrina de la Iglesia y se da un fundamento religioso a la legislación, cosa que sorprende teniendo en cuenta que Bialet Massé se considera a sí mismo socialista. ${ }^{63}$

Apenas hay citas a la doctrina extranjera en su obra. Y cuando las hay, se trata de autores foráneos citados por Vélez Sarsfield, autor del código argentino. $Y$ es que Bialet Massé defendía en esta obra la suficiencia del código argentino para resolver los problemas jurídicos derivados de los accidentes de trabajo. El argentino llega a decir que la obra de Vélez Sarsfield no tenía defectos rechazando de plano cualquier reforma porque «lo que tenemos es bueno y justo». ${ }^{64}$ Tampoco era necesario cambiar nada en el grupo de preceptos que regulaban la responsabilidad civil por daños. En este caso, como en los otros, «no hay código que lo iguale [Al de Vélez Sarsfield] en perfección de ciencia, en previsiones y justicia». ${ }^{65}$ Si Bialet Massé no veía necesario

60 Bialet Massé (1904a). Sobre este informe vid. Murmis (2010). Vid. también el número monográfico de la revista Entrepasados 13 (2004).

61 ZimMERMANN (2013) 85.

62 Bialet Massé (1904b) 2.

63 Bialet Massé (1904b) 4.

64 Bialet Massé (1904b) 8.

65 Bialet Massé (1904b) 15.

$140 \quad$ Alfons Aragoneses 
importar doctrinas o reformar el articulado del código el rechazo a la legislación especial, que podía entrar «por la puerta de la especialidad y los albañales del exotismo», ${ }^{\mathbf{6}}$ era casi visceral.

Bialet Massé defiende una solución doctrinal a la cuestión de los accidentes de trabajo por la vía de la teoría de la sustitución, las reglas sobre el mandato y, aunque sin citarla, la idea de riesgo profesional. La solución por tanto estaba dentro de preceptos concretos del código civil. Según la teoría del argentino-catalán el obrero, al sufrir un accidente, estaba en una situación en la que sustituía al empresario, que era el beneficiario de la exposición al riesgo del obrero. Por ello este último debía ser indemnizado por el empresario. Había así indemnización pero sin culpa.

El rechazo a la legislación especial de Bialet Massé y otros se explica por el particular contexto social y cultural argentino. La aprobación de leyes especiales siguiendo el modelo alemán o el modelo francés respondía a una lógica intervencionista directa o indirectamente provocada por el fantasma del socialismo que Bialet Massé llamaban doctrinario: el socialismo de origen europeo que el argentino oponía a su socialismo de base católica y antimarxista.

Además las leyes especiales rompían la descripción que el liberalismo hacía de sí mismo y rompía la proyección de una sociedad igualitaria, tan importante en el discurso identitario argentino. Estas leyes partían del reconocimiento de la existencia de clases sociales y de lucha de clases, ideas que provocaban conflicto y rompían la idea de la Argentina como tierra de oportunidades para todos. «Huyamos - escribía Bialet Massé - de hacer de la legislación obrera un ramo especial, de excepción, (...), porque tras el obrero está el sirviente; y tras del obrero y el sirviente está el mendigo; no hay derecho obrero porque no hay justicia obrera; hay derecho humano porque hay justicia humana». ${ }^{67}$

Como avanzaba, en Argentina tematizar la lucha de clases o el conflicto social contradecía el credo liberal nacionalista según el cual no existían clases sociales. Como afirma Matthew Karush las elites argentinas negaban la existencia de esta división y lo hacían acentuando la importancia y especificidad de la Nación mediante el uso del concepto de Argentinidad. ${ }^{68}$

66 Ibidem.

67 Bialet Massé (1904b) 24.

68 Karush (2002). Citado en Schjolden (2009) 96. 
En el país americano tenía mucho éxito el discurso según el cual el obrero podía convertirse en empresario ya que el éxito económico era posible para cualquier argentino. Este discurso obviaba los conflictos sociales y servía precisamente para frenarlos al permitir al obrero creer en su promoción social. Al ser Argentina pretendidamente una Nación sin clases, no era necesario un código obrero o una ley específica sobre accidentes de trabajo: el código de Vélez Sarsfield era suficiente para regular las relaciones jurídicas de la sociedad argentina.

La defensa de una solución dentro del código para los accidentes de trabajo está fundamentada, para el caso de Bialet Massé, en la defensa de una sociedad con igualdad formal y que para mantener la paz rechazaba el discurso socialista. Así como la inmigración traía a Argentina ideas socialmente disolventes como el anarquismo, la importación de doctrinas y especialmente normas jurídicas podía también tener un efecto igualmente destructivo de la sociedad argentina, armónica y cohesionada según el discurso liberal. Algunos autores defendían desde las facultades jurídicas la descodificación de las relaciones laborales porque al código le faltaba el espíritu de los nuevos tiempos.

De la recepción de prácticas jurídicas al trasplante legislativo: la ley 9688 de accidentes de trabajo

Pese a esta oposición a la legislación especial sobre accidentes de trabajo se iban redactando proyectos $\mathrm{y}$, paralelamente, se fue extendiendo la práctica por parte de los empresarios de contratar seguros obreros para cubrir las indemnizaciones por accidentes.

El primero de estos proyectos lo presentaron en el congreso los diputados Belisario Roldán y Marco Avellaneda en 1902, sin apenas discusión y nulo éxito. En 1904 Joaquín. V. González presentaba su proyecto de código del trabajo, que incluía obviamente un título dedicado a los accidentes de trabajo que reformaba varios artículos del código civil. En el artículo 89 del proyecto se hablaba de la «obligación empresarial de indemnizar todo accidente sufrido por el obrero por el hecho y en ocasión del trabajo». En 1906 la organización patronal Unión Industrial Argentina presentó también un proyecto de ley para la pública discusión. Pero ninguno de estos proyectos llegó a cuajar aunque sí influyeron discusiones posteriores, especialmente el proyecto de los patronos. 
En 1907 se crea el Departamento Nacional del Trabajo, encargado de estudiar y asesorar al gobierno en una materia que como nos recordaba Caterina, todavía no estaba definida ni «en su denominación, materia, extensión y límites». ${ }^{69}$ Este hecho supuso un importante cambio y un gran impulso para el proyecto de promulgar una ley especial. En la creación de este órgano influyó sin duda la existencia de instituciones similares en Francia y en España pero también las instituciones equivalentes en Estados Unidos y Gran Bretaña. Se trataba de órganos asesores que estudiaban y hacían propuestas. En Argentina se optó por un órgano asesor pero sin capacidad de inspección o sanción.

El mismo año de su creación, el Departamento Nacional del Trabajo presentaba un proyecto de ley sobre los accidentes de trabajo bastante amplio, que incluía algunas enfermedades laborales aunque no imponía el seguro obligatorio. ${ }^{70}$ En 1912 tres diputados socialistas, Palacios, Justo y Laurencena, presentaron un proyecto de ley que preveía un seguro obligatorio. Finalmente, el proyecto que fue discutido y aprobado fue el de 1915, que analizaré más adelante.

Creo conveniente ahora destacar un factor importante en este proceso. Diversos parlamentarios y autores reconocían en esos años que los seguros de accidentes eran ya una práctica extendida en la Argentina. Es más: si hacemos caso de los datos aportados por Alejandro Unsain en su manual de 1915, en 1913 se habían contratado en Argentina seguros de accidentes de trabajo a favor de 51.869 obreros por valor de 80.702 .600 francos. ${ }^{71}$ Miguel Angel Garmendia hablaba de 102.061 obreros, justo el doble, asegurados en 1914. ${ }^{72}$ En Francia, en el año de aprobación de la ley de accidentes de trabajo, los seguros contratados tuvieron un valor de 20 millones de francos. Por tanto había una práctica extendida entre las grandes empresas, seguramente importada por empresas europeas, consistente en asegurar al obrero para evitar el problema económico y social provocado por los accidentes.

Esto es fundamental para entender el éxito del proyecto de 1915 y también para entender que la Unión Industrial Argentina, que agrupaba a las empresas más grandes del país, presentase su propio proyecto de ley en 1906.

69 Caterina (2008) 246.

70 Departamento Nacional del Trabajo (1907).

71 Unsain (1915) 174.

72 Garmendia (1918) 38. 
Ante el avance del socialismo, ante la inevitabilidad de la intervención del Estado, los grandes empresarios apuestan por una norma que impone unas mismas condiciones a todos los industriales y, por tanto, acaba con las ventajas competitivas de los empresarios menos escrupulosos. Además, como señala Zimmermann, los empresarios intentaban que el tema de los accidentes fuese regulado por las asociaciones empresariales, sin la intervención del Estado. Un intento de autorregulación en la línea de la doctrina corporativista. Hay que destacar también que el Departamento Nacional del Trabajo desarrolló, como explica Zimmermann, una campaña de difusión e información en 1907 sobre legislación comparada en la materia. ${ }^{73}$

Este es el contexto de la discusión de la ley de 1915. Los debates parlamentarios están inspirados por la doctrina social de la Iglesia. Se cita expresamente la Encíclica Rerum Novarum y valores como la confraternidad cristiana. Se habla de extirpar una causa de la contradicción entre capital y trabajo. ${ }^{74}$ También se hace referencia a la legislación comparada, básicamente francesa y alemana y a la práctica de las empresas argentinas.

Durante la discusión se reconoce por tanto la contradicción entre capital y trabajo pero se rechaza indirectamente cualquier argumento del socialismo. La ley no se aprueba por la presión del obrerismo, sino por la influencia de la doctrina social de la Iglesia en la práctica económica y jurídica: el Parlamento sencillamente plasmaba en una ley esta realidad social.

El diputado Marcó, representante de la Comisión de legislación encargada de elaborar el proyecto, lo defendió en sede parlamentaria. Habla de la necesidad de superación de la contradicción entre capital y trabajo observando «en primer término, los dictados sugerentes de la experiencia y siguiendo luego el riel que hayan fijado otros pueblos más avanzados que nosotros en materia de legislación social». ${ }^{75}$ Se reconoce por tanto la existencia de la práctica aseguradora antes mencionada. Se debía seguir la legislación social de otros países, pero ante todo consolidando la práctica jurídica. Una práctica que aquí no era producto de una imposición estatal o de la presión del socialismo sino de una actividad espontánea inspirada por la doctrina social de la Iglesia: «Que haya tantos millones en seguros demuestra la verdad de la

73 ZimMERMANN (1995) 195.

74 Diario de Sesiones. Cámara de diputados, 25 de septiembre de 1915, 551.

75 Ibidem. 
doctrina católica: la naturaleza ha sembrado por todas partes la unión y la armonía» Con la ley de accidentes de trabajo, el Parlamento contribuía a «recoger y almacenar ese espléndido sembrado de la naturaleza»: es la naturaleza, la sociedad, la que había sembrado con la unión y la armonía las relaciones económicas.

Desde el ala socialista participó en la discusión el diputado Repetto. Se podría esperar el uso de un lenguaje próximo al socialismo europeo, que propugnaba que el Estado interviniese en la economía para defender a los trabajadores. Encontramos, en cambio, un discurso moderado, coincidente en lo fundamental con el del partido radical. Un ejemplo: Repetto alaba la legislación alemana de accidentes de trabajo y la presenta, no como resultado de la intervención de un Estado modernizador o temeroso del socialismo, como sería imaginable, sino como el fruto de la evolución de las normas gremiales de la Edad Media en Alemania. ${ }^{76}$ Por tanto coincide Repetto con Marcó, en la fundamentación del seguro obligatorio no en la necesidad de que el Estado intervenga siguiendo las demandas de los obreros sino en la solidaridad social y en el ideario corporativo.

Armonía, fraternidad cristiana, gremios medievales, corporativismo son ideas fuerza que están presentes en la Rerum Novarumy que inspiran las intervenciones de parlamentarios de diferentes colores políticos en el parlamento argentino. Encontramos en los debates nacionalismo argentino y religión católico en lugar de socialismo y estatalismo europeos. El resultado final de este debate fue una ley argentina con un texto muy similar al de la ley francesa o española. Pero el debate parlamentario que había tenido lugar en Buenos Aires muestra como los términos utilizados, pese a ser en ocasiones idénticos a los usados en el debate francés, tenían significados muy diferentes.

El proyecto definitivo sería aprobado por el Parlamento en 1915 como Ley 9688 de Accidentes de Trabajo y Enfermedades Profesionales. Si estudiamos su contenido veremos, como comenté, que poco varía respecto a la regulación francesa. Se incluyen solamente los trabajos mecanizados, se define accidente de trabajo como aquel producido durante o como consecuencia del trabajo, dejando a la doctrina y la jurisprudencia la concreción de esta definición. Las cantidades de las pensiones se asemejan bastante: equivalen al

76 Ibidem.

Crisis del derecho privado y legislación especial en Francia y en Argentina 
sueldo medio del trabajador: se pagaban 1000 días de sueldo en caso de fallecimiento o incapacidad total o la merma de sueldo multiplicada por 1000 en caso de incapacidad parcial.

La jurisprudencia en Argentina desarrollaría la ley siempre siguiendo el principio «favor laboris» lo que permitió el desarrollo de un sistema de protección de los obreros que jugaría una función muy importante, como toda la normativa laboral, durante el Peronismo.

\section{Conclusiones: sobre el trasplante del derecho doctrinal y legislado}

A comienzos del siglo XX los juristas eran perfectamente conscientes de la trasformación que sufría el derecho privado y en el carácter global y no solamente nacional de este fenómeno de transformación. Ya se tematizaban la influencia de normas jurídicas, la importación de normas, las traducciones, etc. ... No en vano aparece en aquellos años el derecho comparado moderno.

La evolución del derecho privado en Europa seguía la vía de la socialización y de la superación del individualismo del código francés. Esa tendencia llegó también a América del Sur, algunas de cuyas regiones estaban en plena transformación económica y social. Se reciben materialmente textos de doctrina, de normativa, de política. También se reciben los discursos políticos socialistas, en forma de libros o en forma de conocimientos prácticos traídos por los inmigrantes. El léxico es similar o idéntico al francés o al alemán, no así las propuestas que representan.

Los juristas europeos, los franceses especialmente, eran conscientes de la vida americana que tenían normas e instituciones originarias del viejo continente. Así lo veíamos al recordar el viaje argentino de Duguit y la publicación de sus lecciones. La élite política y económica argentina utiliza la terminología europea para construir autoproyecciones de un determinado sistema social, político y económico, para producir autodefiniciones jurídicas que permitan defender la idea de un Estado liberal y de una sociedad de individuos libres e iguales. La terminología es idéntica, no así la semántica: conceptos como «socialismo», «liberalismo» o «nación» tenían a uno u otro lado del Atlántico significados diferentes, como eran también diversos los contextos social, político e intelectual. Por tanto la importación de textos y discursos europeos en Argentina no podían crear la misma realidad jurídica generada en el viejo continente. 
Estas diferencias las encontramos al abordar el discurso liberal argentino. Como explica Eduardo Zimmermann, el liberalismo programa la intervención del Estado para integrar a una población con una fuerte presencia de inmigrantes y con unas estructuras nacionales a medios construir. ${ }^{77}$ Por ello se importan instituciones que permiten la intervención del Estado, pero precisamente para mantener un statu quo, el liberal, que está amenazado por los conflictos provocados por la industrialización.

El Estado se apoyaba en la ciencia social para desarrollar un programa entre el socialismo de Estado y el laissez faire, siguiendo siempre lo que Zimmermann llama el "pragmatismo»». ${ }^{78}$ A la hora de aprobar la ley de accidentes de trabajo se escogen elementos de normas europeas para conseguir pragmáticamente algunos objetivos. La protección por la ley de la fuerza de trabajo y de su reproducción es innegable. Pero hemos de tener en cuenta que en Argentina no dejaban de llegar barcos con inmigrantes y que el Parlamento se permitía aprobar normas para expulsarlos en casos de huelgas o manifestaciones. ${ }^{79}$

Otro de los objetivos del Estado al aprobar la Ley fue sin duda acabar con las desventajas competitivas de las grandes empresas que motu proprio aseguraban a los trabajadores. Las empresas que tenían contratados seguros tenían un coste laboral adicional que debían repercutir en los beneficios o en los precios. Esto las situaba en inferioridad de condiciones a la hora de competir con otras empresas. Las desventajas competitivas ponen en riesgo el mercado según lo concibe la doctrina liberal. Por ello tiendo a pensar que la razón no era tanto la paz social sino la paz empresarial: la Unión Industrial Argentina presiona al parlamento para que imponga la obligatoriedad de los seguros como medio de eliminación de las desventajas competitivas de las empresas que aseguraban a los trabajadores frente a las que no se ahorraban ese gasto.

En definitiva, podemos concluir afirmando que, en el proceso de transformación del derecho privado en Argentina de comienzos del siglo XX, se

ZimMERMANN (1995).

78 Zimmermann (1995).

79 La ley 4144 de Residencia fue aprobada en 1902 y permitía expulsar a extranjeros y se aplicó para descabezar el movimiento obrero. Se aplicó a personas como Francesc Macià, líder político y primer Presidente de la Cataluña autónoma de la II República. Alfredo Palacios acogió y defendió como abogado al que sería primer presidente de la Cataluña republicana. Sobre la ley y el caso Macià-Gasol vid. Aspell de Yanzi Ferreira (1987). 
utilizan elementos del lenguaje jurídico europeo. Es decir: doctrinas, nombres, ideas que habían llegado a Argentina desde España o Francia. En cambio, los modelos sociales y políticos en que se sostiene este lenguaje son diferentes. También lo es el significado cultural de determinadas experiencias jurídicas. Así, el código civil argentino sirve para proyectar un modelo de sociedad que integra a la pluralidad de grupos humanos del país y las leyes especiales Encontramos, como diría António Hespanha, continuidades o similitudes terminológicas, pero no semánticas: «Saleilles», «Duguit», «doctrina social de la Iglesia» 0 «leyes de accidentes» en «argentino» no significan lo mismo que en «castellano europeo». ${ }^{80} \mathrm{La}$ historia de estos procesos debe tener en cuenta estas diferencias en el significado de expresiones y por tanto también de documentos considerados iconos por la historiografía.

Pero esto también nos trae otra conclusión: el estudio del derecho europeo de esa época pero también de épocas posteriores debe tener en cuenta sus aspectos culturales y también sus aspectos políticos, pues todo proyecto jurídico contiene también un proyecto político. En el momento actual en el que vence el discurso de la neutralidad y objetividad del derecho que regula las relaciones laborales y económicas, triunfa también la fe en el trasplante de unas reglas de intercambio neutras y objetivas que responden a la naturaleza de las cosas y no a relaciones de poder. Ahora, como hace cien años, esos discursos se visten con el lenguaje de la modernización, como si hubiese solamente una sola vía a la modernidad y como si esta no tuviese carga política e ideológica.

Precisamente por ello es útil revisitar con nuevas herramientas la historia de la segunda globalización del derecho contemporáneo, la que se dio justo antes de la I Guerra Mundial que ahora conmemoramos. 


\section{Bibliografía}

Aragoneses, Alfons (2009), Un jurista del modernismo. Raymond Saleilles y los orígenes del derecho comparado, Madrid

Arnaud, André-Jean (1973), Essai d'analyse structurale du Code civil français: la règle du jeu dans la paix bourgeoise, Madrid

Aspell de Yanzi Ferreira, Marcela (1987), Expulsión de extranjeros. La ley 4144 «de residencia» y la jurisprudencia de la Suprema Corte de Justicia de la Nación, in: Revista de historia del derecho 15, 9-100

Bialet Massé, Juan (1904a), Informe sobre el estado de las clases obreras en el interior de la República, Buenos Aires (Edición en Internet en la URL http:// www.trabajo.gba.gov.ar/informacion/masse/Volumen1.pdf)

Bialet Massé, Juan (1904b), Tratado de la responsabilidad civil en derecho argentino bajo el punto de vista de los accidentes de trabajo, Rosario de Santa-Fe

Caterina, Luis María (2008), Presentación a: La construcción de una nueva disciplina. El derecho del trabajo, in: Tau Anzoátegui, Víctor, Antología del pensamiento jurídico argentino (1901-1945), Buenos Aires, 243

Cazzetta, Giovanni (1991), Responsablità aquiliana e frammentazione del diritto comune civilistico (1865-1914), Milano

Cheysson, Emile (1889), Organisation de l'assurance contre les accidents, in: Gruner (1889) 451-477

Decouflé, André-Clément (1984), Histoire de l'office du travail. Une administration de mission avant la lettre, in: Revue Travail et Emploi 22, 45-55

Departamento Nacional del Trabajo (1907), Reparación de los accidentes del trabajo. Exposición y proyecto de Ley, Buenos Aires

Droz, Numa (1889), Etat de la question des accidents du travail en France et à l'étranger, in: Gruner (1889) 9-44

Duguit, LÉon (1912), Las transformaciones generales del Derecho privado desde el Código Napoleón, Madrid

Duguit, LÉon (1913), Les transformations générales du droit privé depuis le Code Napoléon, Paris

Duve, Thomas (2012), Von der Europäischen Rechtsgeschichte zu einer Rechtsgeschichte Europas in globalhistorischer Perspektive, Frankfurt am Main

Garmendia, Miguel Angel (1918), Jurisprudencia del trabajo. Exposición y crítica, Buenos Aires

Giordano, Christian (1996), The past in the present: actualized history in the social construction of reality, in: Focaal. European Journal of Anthropology 26/27, 97-107

Grossi, Paolo (2000), Scienza giuridica italiana. Un profilo storico, 1860-1950, Milano

Gruner, Edouard (dir.) (1889), Congrès international des accidents du travail et des assurances sociales, Paris 
Hakim, Nader (2004), Les premiers traités de législation industrielle et la jurisprudence: les ressources d'une influence doctrinale au Palais, in: Le Crom (2004) 29-39

Halpérin, Jean-Louis (1996), Histoire du droit privé français depuis 1804, Paris

Herrera, Carlos Miguel (2011), Entre équité et socialisme? Le juge et la question sociale dans le débat politico-doctrinal français du début du XXe siècle, in: Quaderni Fiorentini per la storia del pensiero giuridico moderno 40, 331-366

Hespanha, António Manuel (2000), Cultura jurídica europea. Síntesis de un milenio, Madrid

Hordern, Francis (2001), De la législation industrielle au droit du travail (1890-1960), in: Construction d'une histoire du droit du travail, Cahiers de l'IRT 9, 43-56

Jamin, Christophe, Pierre-Yves Verkindt (2003), Droit civil et droit social: l'invention du style néoclassique chez les juristes français au début du vingtième siècle, in: Kasirer, Nicholas (dir.), Le droit civil, avant tout un style?, Montréal, 101-120

Karush, Matthew B. (2002), Workers or Citizens: Democracy and Identity in Rosario, Argentina (1912-1930), Albuquerque

Le Crom, Jean-Pierre (dir.) (2004), Les acteurs de l'histoire du droit du travail, Rennes

Levaggi, Abelardo (2006), Historia del Derecho del Trabajo argentino y sus fuentes (1800-2000), in: Iushistoria. Revista electrónica 3, 59 y 60

Lloyd, Reginald (dir.) (1911), Impresiones de la República Argentina en el siglo veinte, Londres

Morris, James O. (1966), Elites, Intellectuals, and Consensus: A Study of the Social Question and the Industrial Relations System in Chile, Ithaca

Murmis, Miguel (2010), El mundo del trabajo: Juan Bialet Massé como precursor y contemporáneo, Buenos Aires

Oddone, Jacinto (1984), Historia del Socialismo argentino (1896-1911), Buenos Aires

Quesada, Ernesto (1906), La facultad de derecho de París, estado actual de su enseñanza, Buenos Aires

Ramacciotti, Karina InÉs (2014), Influencias internacionales sobre la gestión de los accidentes de trabajo en Argentina. Primera mitad del siglo XX, in: Revista electrónica de estudios latinoamericanos 12, 1-22

Sainctelette, Charles (1886), Accidents de travail. Projet d'une proposition de loi, Bruxelles

Saleilles, Raymond (1897), Les accidents de travail et la responsabilité civile, Paris

Saleilles, Raymond (1898), Le risque professionnel dans le code civil, in: Réforme sociale 35, 634-665

Saleilles, Raymond (1904), Méthode historique et codification, in: Atti del Congresso Internazionale di Scienze Storiche, Roma, 3-22

Saleilles, Raymond (1911), Allocution, in: Les méthodes juridiques. Leçons faites au Collège libre des Sciences sociales en 1910, Paris, XV-XXV 
Schjolden, Line (2009), Sentencing the Social Question: Court Made Labour Law in Cases of Occupational Accidents in Argentina, 1900-1915, in: Journal of Latin American Studies 41, 91-120

Soubiran-Paillet, Francine (2004), De la Société d'études législatives au Conseil national économique: production savante ou production par des acteurs collectifs du droit du travail?, in: Le Crom (2004) 69-82

Tarbouriech, Ernest (1889), Des assurances contre les accidents du travail. Assurance collective et de responsabilité civile. Thèse pour le doctorat soutenue le jeudi 27 juin 1889, Besancon

Tarbouriech, Ernest (1896), La responsabilité des accidents dont les ouvriers sont victimes dans leur travail. Histoire, jurisprudence et doctrine. Bibliographie. Travaux parlementaires jusqu'à la date du 24 mars 1896, Paris

Tau Anzoátegui, Víctor (2008), La Codificación en la Argentina, 1810-1870. Mentalidad Social e Ideas Jurídicas, Buenos Aires

Thaller, Edmond (1914), Avant-Propos, in: L'œuvre juridique de Raymond Saleilles, Paris, 5-38

Unsain, Alejandro M. (1915), Manual de la legislación obrera argentina, Buenos Aires

Vielfaure, Pascal (2005), Note introductive au corporativisme de Vichy, in: Mazzacane, Aldo et al. (Hg.), Korporativismus in den südeuropäischen Diktaturen, Frankfurt am Main, 9-34

Watson, Allan (1993), Legal Transplants. An Approach to Comparative Law, Athens and London.

Zeballos, Estanislao (1919), Cuestiones y legislación del trabajo, in: Revista de la Universidad Nacional de Córdoba 6, 4-6

Zimmermann, Eduardo (1995), Los liberales reformistas. La cuestión social en Argentina 1890-1916, Buenos Aires

Zimmermann, EduARdo (2005), Libéralisme et sciences sociales en Argentine à la fin du dix-neuvième siècle: des Lumières à la nouvelle "connaissance sociale», in: Amérique Latine. Histoire et Mémoire. Les Cahiers ALHIM, 11

Zimmermann, Eduardo (2013) «Un espíritu nuevo»: la cuestión social y el Derecho en la Argentina (1890-1930), in: Revista de Indias 73, 81-106 



\title{
Institucionalización de la Reforma Agraria (1915-1937)
}

\author{
Revolución y modernización jurídica en México
}

Este artículo es un primer intento por resolver un cruce de caminos planteado en la última década del siglo XIX y las tres o cuatro primeras del siglo XX. En ese cruce, uno de los caminos sería el construido por las problemáticas sociales y políticas, en particular su vertiente agraria - generalmente aducidas como una de las causas de descontento que condujeron a la crisis del régimen porfirista -, y las propuestas de solución articuladas por la Revolución Mexicana. ${ }^{1}$ El segundo, las evoluciones del derecho occidental - en particular el derecho privado y, sobre todo, el derecho de propiedad. En ese cruce, la reforma agraria revolucionaria y la Constitución de 1917 han sido vistas, algunas veces, como precursoras de desarrollos políticos y jurídicos posteriores en el resto de Occidente. ¿En qué medida fue así? ¿La coyuntura política y social mexicana efectivamente generó innovaciones que aceleraron el propio desarrollo jurídico occidental, o que al menos influenciaron otras reformas agrarias en Latinoamérica? ¿O esto fue evaluado así a posteriori como parte de la mitología nacionalista sobre la revolución?

Parte de esa mitología es nombrar Revolución Mexicana (en singular) a la variedad de movimientos que, con distinta composición social e ideología, terminaron por derrocar la dictadura de Porfirio Díaz, así como adscribirle de origen las banderas de justicia social por la que será conocida a partir de 1917. Sin embargo, al justificar el levantamiento armado por la opresión política y la desigualdad social del régimen vencido, las facciones triunfantes hubieron

1 La etapa de lucha armada de la Revolución inició en noviembre de 1910 con el levantamiento de Francisco I. Madero contra el régimen del presidente Porfirio Díaz, y se prolongó por las luchas entre las distintas facciones hasta 1919. Se considera la década de 1920 como los años de reconstrucción y, en general, se da por concluida la institucionalización del nuevo régimen político con la presidencia de Lázaro Cárdenas (1934-1940). Existe una amplísima bibliografía al respecto, dos obras generales de provechosa consulta pueden ser: KNIGHT (1986); y los capítulos correspondientes de Velazquez et al. (2010). 
de sostener los ideales liberales de la Reforma que decían querer recuperar (un programa democrático, secularizador y anticorporativo, basado en el positivismo jurídico y el individualismo propietario, plasmado en la constitución federal de 1857), al mismo tiempo que era necesario premiar a las bases campesinas que habían hecho posible el triunfo, reconociendo su demanda de redistribución agraria. Esta contradicción de origen persiste en las dos décadas que pretendemos analizar. Mientras que la breve presidencia de Francisco I. Madero - quien había llamado a la insurrección - se centró en el elemento democratizador y de apego a la ley, los generales sonorenses Venustiano Carranza y, en particular, Álvaro Obregón - optaron por construir las bases corporativas de una nueva legitimidad, sin embargo sin romper con el liberalismo iusnaturalista decimonónico.

Es así que durante la presidencia de Carranza se expide la primera Ley Agraria (6 de enero de 1915) que dispuso el reparto y restitución de tierras a los pueblos que las hubieran perdido, misma que fue ratificada y ampliada en la Constitución federal de 1917, en cuyo artículo 27 se estableció la propiedad originaria de la nación sobre el suelo y el subsuelo y restableció el pluralismo jurídico al reconocer la propiedad colectiva (que había sido abolida por la anterior constitución liberal de 1857). Este fue el puntapié inicial del programa institucional de reforma agraria (algunos generales habían hecho repartos limitados, por propia iniciativa, en las regiones que controlaban) que se desarrolló, con marchas y contramarchas, profusamente en el plano legal hasta la promulgación del Código Agrario en 1934 y su reforma en 1937. En la práctica, sin embargo, Carranza y Obregón distribuyeron poca tierra y no perjudicaron a la clase terrateniente, mientras que Plutarco Elías Calles pretendió dar por terminado el reparto en 1925. Es durante la presidencia de Lázaro Cárdenas (1934-1940) cuando comenzó el periodo de distribución masiva de tierras, junto con un completo programa agrario que implicó la corporativización del campesinado, el desarrollo de la organización administrativa ejidal (distinta y paralela a la municipal), la educación rural socialista, el crédito ejidal, etc. Dos reformas constitucionales importantes de ese mismo año de 1934 quitaban cualquier recurso jurídico a los propietarios afectados por la reforma agraria y anulaba el carácter vitalicio de las magistraturas, es decir, abolía la inamovilidad de los jueces, lo que permitió al presidente nombrar a la Suprema Corte que le acompañó en su sexenio. Por supuesto, ambas reformas, junto a la voluntad de Cárdenas, son las que permitieron el reparto de casi 18 millones de hectáreas entre 815.000 beneficiarios durante su presidencia. 
Este breve repaso nos permite ubicar las problemáticas principales que abordaremos en este trabajo propuesto:

En primer lugar, las continuidades y rupturas en la temporalidad mexicana. Sabemos que en los últimos lustros del régimen porfirista surgen voces críticas contra los efectos de la reforma liberal individualista sobre la propiedad campesina e indígena (las más notorias: Wistano Luis Orozco, 1895 y Andrés Molina Enríquez, 1909), así como sobre las dificultades para las asociaciones de beneficencia laicas (LiRA, 2009). Al mismo tiempo, hay reformas legislativas que implican cambios en este sentido, y aparecen voces entre los hombres de leyes sobre nuevas maneras de entender la sociedad y el derecho. Subsecuentemente, las décadas de 1910 y 1920 (las presidencias de Madero, Huerta, Carranza y Obregón), muestran más acomodos políticos que cambios de fondo. Esto nos obliga a, antes que nada, sistematizar los cambios legislativos referentes a la propiedad agraria desde 1911 hasta 1937, para identificar cambios y permanencias en el régimen de propiedad sostenido por los sucesivos gobiernos revolucionarios y, por el otro, sondear en la prensa general y especializada y en la bibliografía jurídica de la época los temas, los personajes y los debates en el campo jurídico.

En segundo lugar, cabe entonces indagar en qué medida los desarrollos jurídicos del periodo considerado responden meramente a las circunstancias locales y políticas y en qué medida están también influenciados por, e influencian a, procesos extra-mexicanos, tanto en el plano político y socioeconómico como propiamente en el campo del derecho. Resulta obvio que las pocas características mencionadas del sexenio cardenista responden no solamente a los procesos políticos mexicanos sino más ampliamente a la categoría que ha sido definida como «Estado intervencionista», ${ }^{2}$ y a las características de lo que, de Comte a Duguit pasando por Durkheim, se llamaba entonces «socialización del derecho", también en México. ${ }^{3}$ Pero ¿qué sucedía en las dos décadas previas, en el periodo que hemos definido entre 1915 y 1934? ¿Qué desarrollos jurídicos son propios del caso mexicano? ¿En qué medida la reinstauración de la propiedad corporativa o del pluralismo propietario supuso una modernización jurídica? ¿Qué elementos de la reforma agraria mexicana, y las adecuaciones al sistema jurídico que la hicieron posible, son discutidas y recogidas en otros países latinoamericanos o europeos? ¿Cuáles fueron

2 Mannori, Sordi (2004).

3 Olea y Leyva (1933). 
tomadas de teóricos del derecho o de procesos simultáneos de otros países? «No nos es posible contestar aún todos estos interrogantes, pero en estas páginas estableceremos los cambios legislativos y constitucionales, así como los temas problematizados por la prensa de la época. En una próxima etapa, ahondaremos en las tesis de licenciatura en derecho, prensa y bibliografía jurídica de la época para concretar el proyecto que estamos proponiendo.

\section{La propiedad de la tierra y sus reformas jurídicas}

México ha sido un país fundamentalmente agrario. Todavía hasta la década de 1930 el sector agropecuario representaba la porción principal del producto interno bruto y, en ese año, las dos terceras partes de la fuerza de trabajo residían en el campo. Dicho esto, analicemos los diferentes sistemas agrarios que estuvieron vigentes: Después de la conquista española, la cual incorporó los sistemas nativos al mercantilismo europeo, reconocemos otros dos hitos legales que impactaron el sistema de propiedad de la tierra, y que, no es casualidad, coincidieron con las dos mayores guerras civiles que ha sufrido México, motores de transformaciones determinantes en la historia nacional, tanto en el régimen de gobierno como en la composición de las élites y, por supuesto, en la conformación del México moderno.

El primero de estos cambios legales fue el programa liberal decimonónico, que se propuso forjar una sociedad de mercado, formada por individuos productores y consumidores, con derechos políticos y de propiedad personales, eliminando a las corporaciones. Se consideraba que el pequeño y mediano productor debía ser el actor principal de la modernización agraria y la constitución de un mercado libre de tierras, en oposición a una realidad donde la tierra permanecía estancada en manos corporativas (sobre todo de la Iglesia y las comunidades indígenas), o inculta en grandes latifundios improductivos o baldíos no identificados por el Estado. Estas ideas sociales y económicas guiaron la política agraria desde 1856 (fecha de expedición de la ley federal de desamortización de corporaciones civiles y eclesiásticas) hasta la primera década del siglo XX, y se tradujeron en un profuso corpus legal [artículo 27 de la constitución de 1857, leyes sobre nacionalización de bienes y capitales eclesiásticos (1859), colonización, deslinde y tierras baldías (1863, 1883, 1894), varios decretos del ejecutivo ordenando la desamortización de ejidos comunales y municipales (1889-1890), y la codificación civil que inició en 1868] y jurisprudencial que transformaron radicalmente el sistema jurídico 
de la propiedad, para establecer el monismo legal de la propiedad privada «perfecta» (deslindada y titulada, sin obstáculos para incorporarse al mercado libre de tierras), favorecer la inversión de capitales y obtener ingresos públicos.

Con el cambio de siglo, el positivismo permitió algunas atenuaciones en las disposiciones que privaban de personalidad jurídica a las corporaciones civiles: ésta les fue devuelta temporalmente a los ayuntamientos en la ley de tierras baldías de 1894 (con el objeto de que resolvieran judicialmente los límites y derechos de propiedad en litigio entre pueblos, para poder proceder a su reparto), y en 1901 se reformó el artículo 27 constitutional para habilitar y permitir a las corporaciones civiles desvinculadas de instituciones religiosas la posesión de tierras y la administración de hipotecas (aunque esta última medida estuvo motivada por la problemática de las asociaciones de beneficencia). ${ }^{4}$ Una ley, en noviembre de 1896 , cedía gratuitamente a los campesinos pobres los terrenos baldíos o nacionales que estuvieran ocupando, así como a nuevas poblaciones para conformar su fundo legal y para garantizar servicios públicos. El reglamento de septiembre de 1897 consideraba como sujetos de la ley recién citada a quienes ocuparan terrenos baldíos o nacionales con valor igual o menor a $\$ 200$. No obstante, también afirmaba que los ejidos y terrenos de común repartimiento no eran objeto de dicha ley y se seguirían parcelando y adjudicando. En diciembre de 1902 un decreto presidencial sometía los futuros deslindes de tierras bajo el control directo de Porfirio Díaz, y exigía como requisito de propiedad el ocupar la tierra por treinta años. Tres decretos en 1909 suspendieron la venta de tierras baldías indefinidamente y prohibieron la renovación de los contratos una vez expirada la concesión original. Finalmente, en abril de 1911 el presidente Díaz sugirió al Congreso se considerara la adopción de políticas federales de fraccionamiento de latifundios y de irrigación en gran escala, para beneficiar la pequeña y gran producción agrícola empresarial.

El contexto de estas últimas medidas, que de todos modos no se alejan del liberalismo dominante, lo constituye una sucesión de crisis económicas y agrícolas en 1907-1910, así como las nuevas corrientes que discutían los operadores jurídicos y la propia crisis del régimen porfiriano que, iniciado en 1876, llevaba ya tres décadas de dictadura y estaba mostrando su agotamiento, en particular por el surgimiento de voces críticas que pedían cambios sociales y políticos.

4 Lira (2009). 


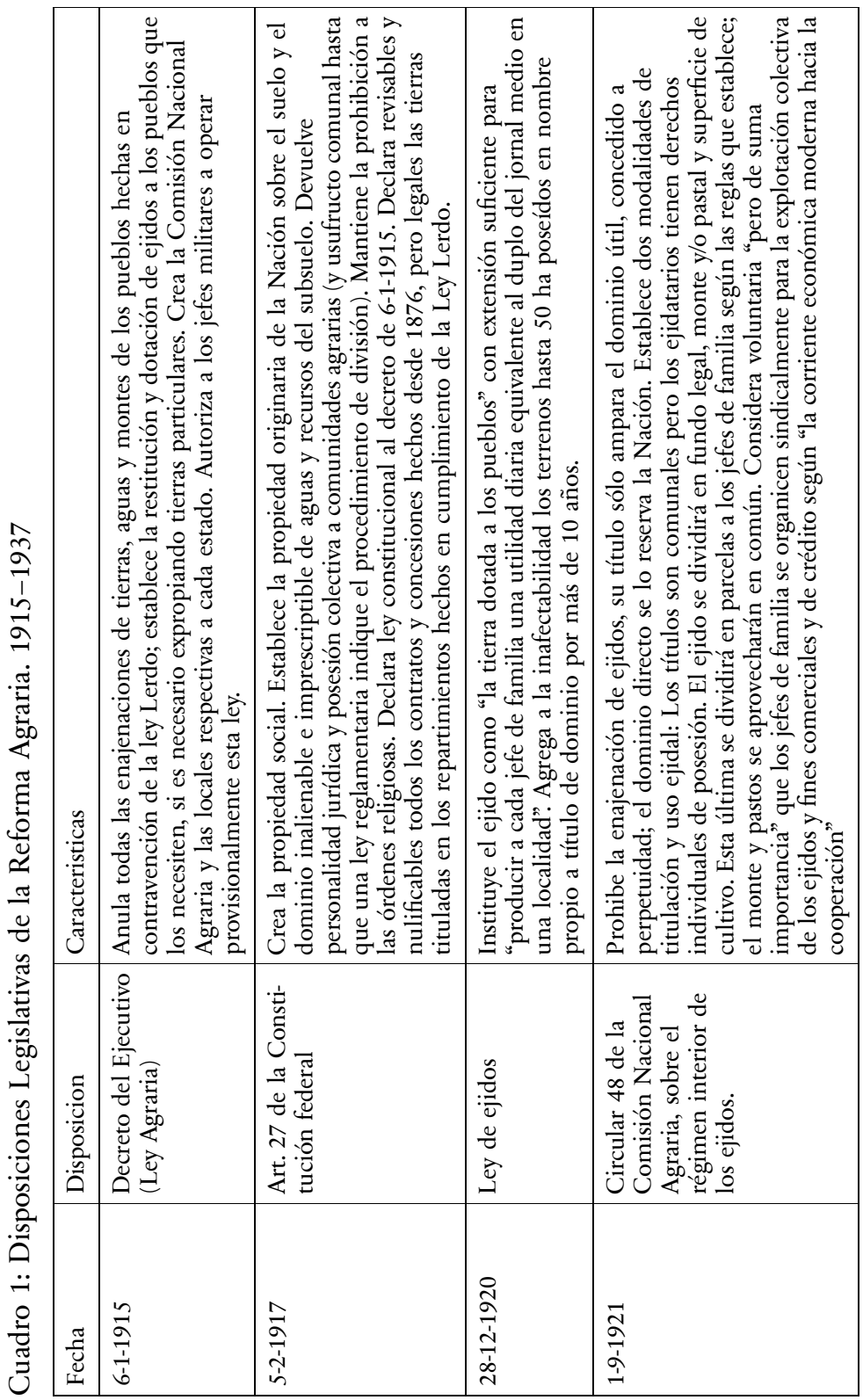




\begin{tabular}{|c|c|c|c|c|c|c|c|}
\hline 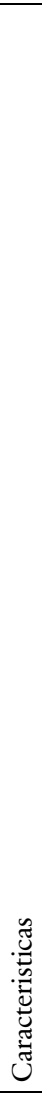 & 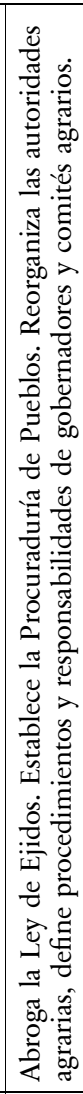 & 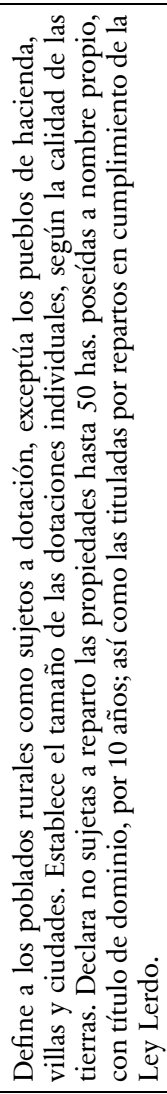 & 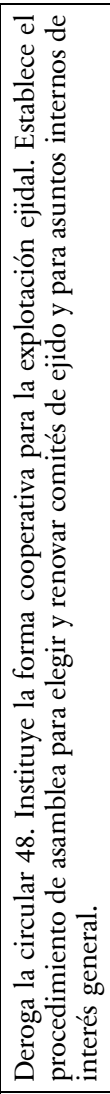 & 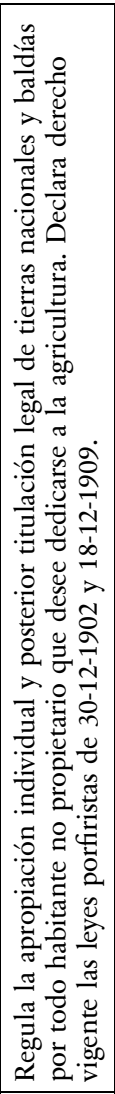 & 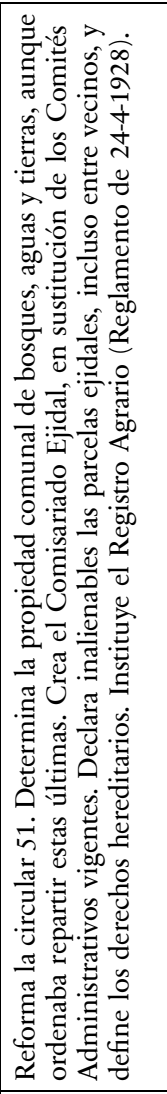 & 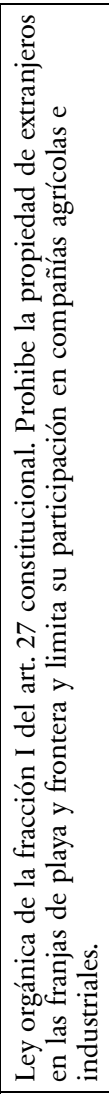 & 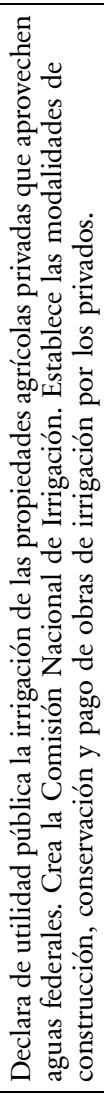 \\
\hline $\begin{array}{l}\frac{0}{0} \\
\frac{u}{0} \\
0 \\
\frac{0}{n} \\
0\end{array}$ & $\begin{array}{l}\stackrel{0}{0} \\
\stackrel{0}{0} \\
0\end{array}$ & 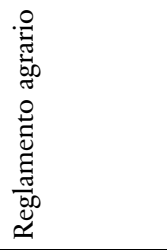 & 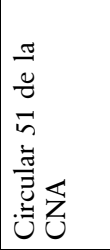 & 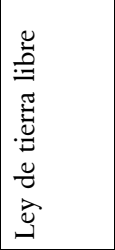 & 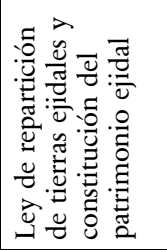 & 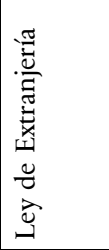 & 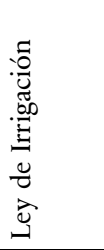 \\
\hline$\frac{\pi}{\tilde{U}}$ & 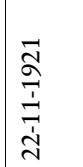 & $\frac{\pi}{2}$ & $\frac{i}{2}$ & 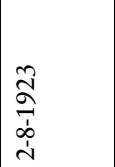 & 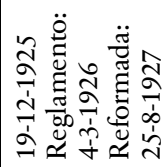 & $\frac{a}{a}$ & $\frac{\widetilde{T}}{\frac{\pi}{+}}$ \\
\hline
\end{tabular}




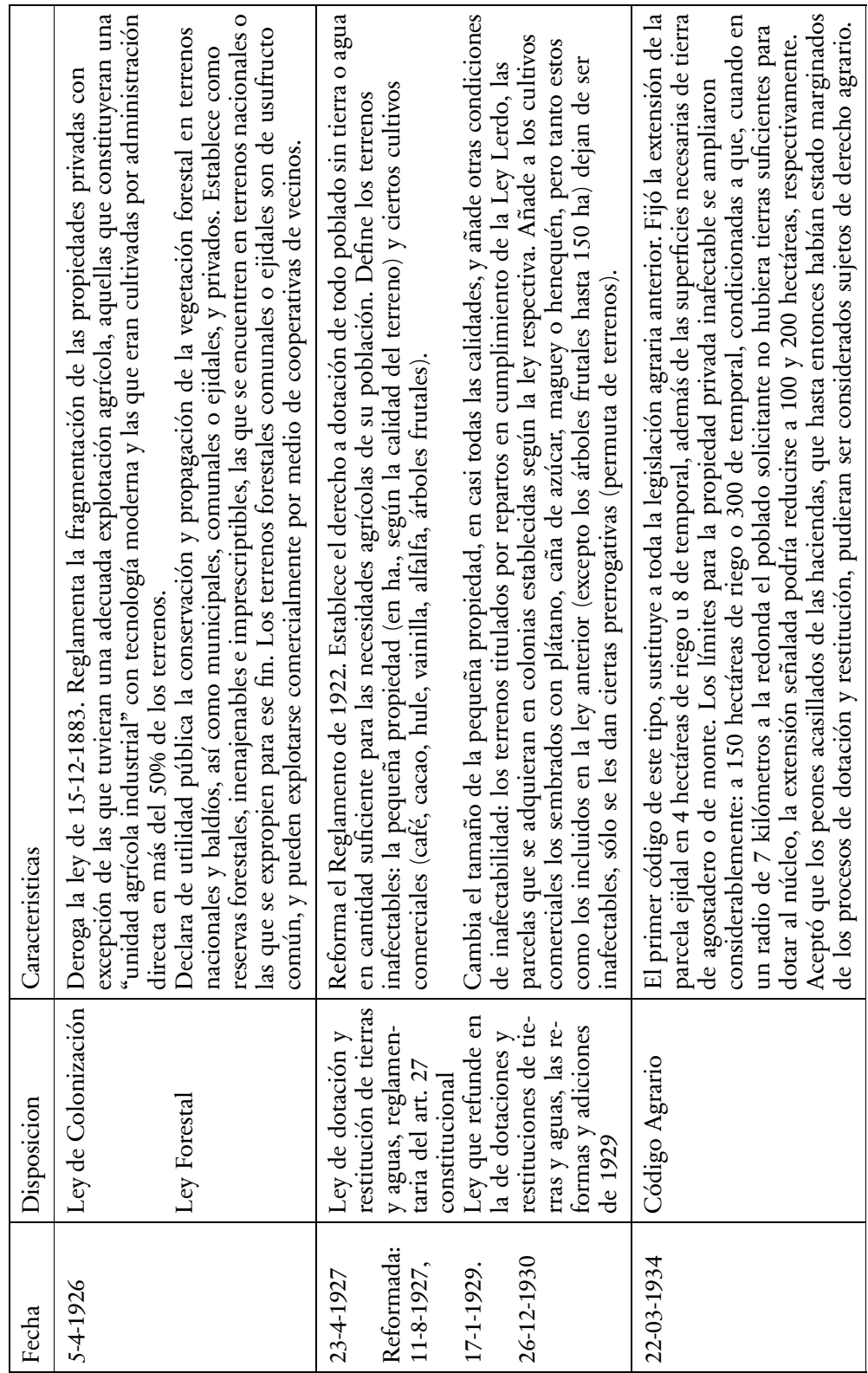




\begin{tabular}{|c|c|c|}
\hline & 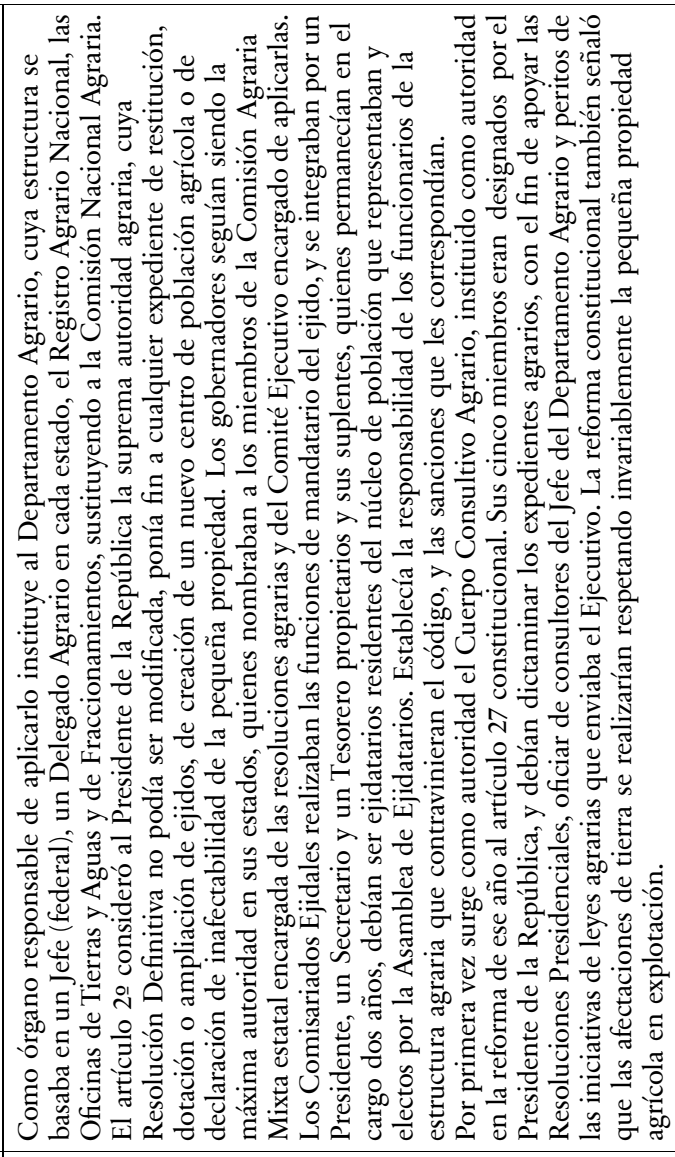 & 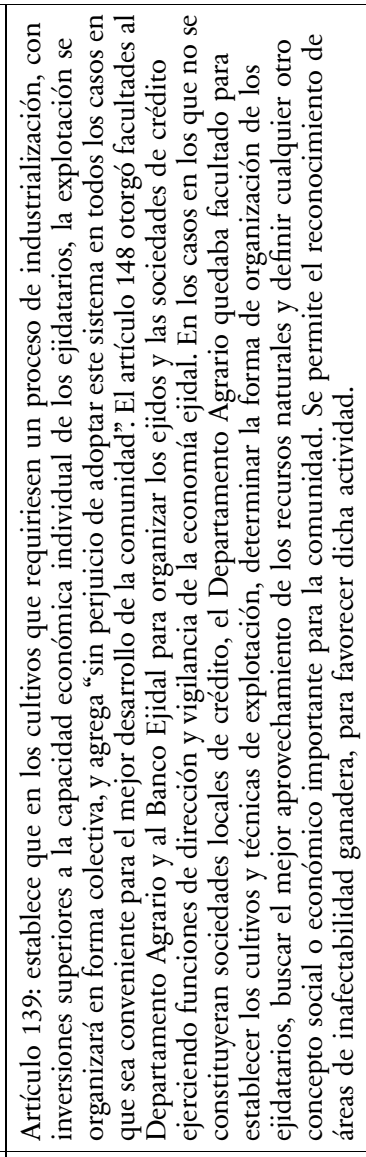 \\
\hline & 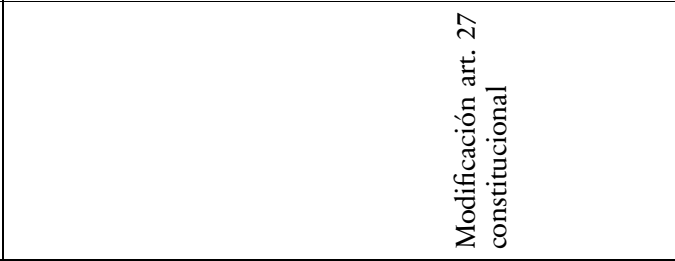 & 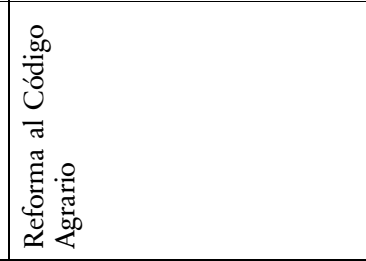 \\
\hline $\begin{array}{l}\tilde{J} \\
\text { U్ } \\
\text { I }\end{array}$ & 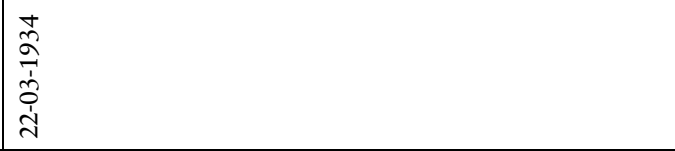 & $\frac{\bar{d}}{b}$ \\
\hline
\end{tabular}


La segunda gran transformación jurídica del sistema de propiedad de la tierra en el México moderno la concretó la Revolución Mexicana, por medio de su reforma agraria, misma que detallamos en el cuadro 1. Esta inició con la sanción de la Ley Agraria de 6 de enero de 1915 y el artículo 27 de la constitución de 1917, medidas ambas que reintrodujeron, aunque sobre bases distintas a la colonial, un sistema jurídico plural de propiedad de la tierra, al reconocer la legalidad de la propiedad colectiva y el derecho originario del Estado sobre todo el territorio.

La ley de 6 de enero de 1915, promulgada por Venustiano Carranza, instituyó los mecanismos legales, institucionales y administrativos de la reforma agraria. Abolió las enajenaciones de tierras, aguas y montes pertenecientes a los pueblos, que se hubieran hecho contraviniendo la ley de desamortización de 1856, y fijó "como acto de elemental justicia» su devolución, así como el otorgamiento de más tierras a los pueblos que justificaran su necesidad, mediante los mecanismos de restitución y dotación, respectivamente. Posteriormente, el artículo 27 de la Constitución de 1917 instauró la propiedad original de la Nación sobre el suelo, el subsuelo y las aguas, y abolió el monismo legal de la propiedad que había introducido la constitución anterior de 1857, al reconocer jurídicamente tanto la propiedad privada (restringida por el derecho de expropiación por causa de utilidad pública) como la propiedad colectiva (creada por los mecanismos de dotación y restitución de la ley de 1915 , a partir de la expropiación a la primera). ${ }^{5} \mathrm{La}$ Constitución dejaba en manos de los estados las tareas de fraccionar la gran propiedad expropiada y de fijar en leyes agrarias la extensión máxima de tierras en manos de un propietario.

No obstante, se ha señalado que el cambio de rumbo de la última década porfirista y el personal que la pensó e instrumentó, tuvo cierta continuidad después de la revolución y hasta 1930, pese a que, paralelamente, comenzaba la instrumentación del reparto agrario y un giro más acusado en el discurso ideológico del gobierno. ${ }^{6} \mathrm{Y}$ coincidimos desde que una circular carrancista, del 21 de marzo de 1918, afirmaba que no obstante que era «urgente» proveer de tierras para sembrar a los labradores pobres, eso no sería posible hasta que se reglamentara el artículo 27 constitucional, y sólo se podían otorgar tierras de acuerdo a la Ley de 29-11-1896 y su reglamento de 6-9-1897; mientras que

5 Marino y Zuleta (2010).

6 García Ugarte (1995). 
el presidente Obregón, en la Ley de Tierra Libre (2-8-1923), invoca como fuente jurídica vigente («que subsiste en toda su amplitud») las leyes de 30-121902 y de 18-12-1909 al sostener las atribuciones del Estado federal para disponer en materia agraria y, en particular, de las tierras baldías y nacionales.

Más aún, García Ugarte sostiene que «en 1910, la Secretaría de Fomento porfirista empezó a reconocer la necesidad de crear la pequeña propiedad o propiedad de familias [...] y fomentar un fraccionamiento natural del latifundio mediante políticas arancelarias y productivas que condujeran a la baja el valor de la propiedad raíz», política que habría sido retomada por personajes importantes de los primeros años del nuevo régimen. ${ }^{7}$ Hay consenso en la historiografía sobre la Reforma Agraria en señalar que no sólo maderistas o carrancistas, sino en general los presidentes hasta 1934 coincidieron en mantener la gran propiedad, en particular la orientada a la exportación, para sostener la productividad y los ingresos fiscales, aunque se vieron forzados, periódicamente, a repartir tierras a los grupos que seguían movilizándose reclamando el pago al apoyo prestado en la revolución. Algunos incluso sostienen que los frecuentes cambios legislativos entre un presidente y el siguiente (ver cuadro 1), y muy en particular entre Carranza y Obregón, tuvieron como fin deliberado el nulificar las numerosas solicitudes de dotación. Lo cierto es que la profusa y complicada legislación, así como la tramitología que establecía y los sucesivos cambios en las autoridades del reparto, respondieron muy bien al escaso convencimiento del ejecutivo en que la expropiación y reparto de los latifundios fuera a aportar el crecimiento económico.

Así, la legislación de la reforma agraria, al menos hasta 1930, dio continuidad al cambio instituido en la última década porfirista, no afectó tierras particulares en los pueblos que hubieran sido tituladas como resultado de procesos legales de reparto aplicando la ley de desamortización ni, hasta 1934, afectó haciendas u otras unidades productivas para constituir y dotar pueblos de hacienda, de modo de no dejarles sin mano de obra, sino sólo para restituir ejidos y fundo legal a los pueblos colindantes que los necesitaren. No obstante, desde el punto de vista del impacto económico causado por la certeza jurídica de la titulación, debemos afirmar que la reforma agraria revolucionaria, al reintroducir, sobre nuevas bases, el pluralismo jurídico de la propiedad definiendo la legalidad de la propiedad colectiva inenajenable (en sus

\section{Ibídem.}


dos variantes: las comunidades que habían logrado subsistir a la reforma liberal y el nuevo ejido) junto a la propiedad privada, añadió nuevos títulos legítimos de propiedad, complicando el panorama jurídico. Esto, sin entrar a los modos en que se realizó dicho reparto, sobre todo en la fase armada en que se facultó a los jefes militares para instrumentar expropiaciones y dotaciones, quienes muchas veces lo hicieron sin cuidar los formalismos legales; a los vaivenes de la legislación agraria, particularmente en el periodo 1915-1930, que modificaron criterios y procedimientos con bastante frecuencia; y a la práctica de otorgar títulos de dotación aunque no hubiera tierras que entregar en algunos casos (dotación «virtual»), así como de entregar las tierras antes de resolver todas las instancias jurídicas (alguna de las cuales podía ordenar revertir el proceso), lo que ponía en contradicción títulos legales con usufructo y generaba violencia entre los actores agrarios.

Cuadro 2: Reparto agrario, por periodos y tipo de tierra (en hectáreas)

\begin{tabular}{|r|r|r|}
\hline Periodo & Dotaciones & Beneficiarios \\
\hline $1900-1914$ & 142 & 11738 \\
\hline $1915-1934$ & 7000 & 866161 \\
\hline $1935-1940$ & 10975 & 728847 \\
\hline Totales & 18117 & 1606746 \\
\hline
\end{tabular}

\begin{tabular}{|r|r|r|r|r|r|r|r|}
\hline \multirow{2}{*}{ Periodo } & \multicolumn{7}{|c|}{ Tipo de Tierra (hectáreas) } \\
\cline { 2 - 9 } & Total & Riego & Temporal & Agostadero & Monte & Desértica & Indefinida \\
\hline $1900-1914$ & 194495 & 27194 & 17976 & 58749 & 18399 & 16987 & 55190 \\
\hline $1915-1934$ & 11580833 & 247645 & 1387258 & 2046772 & 1509623 & 189376 & 6200159 \\
\hline $1935-1940$ & 18786131 & 937401 & 3382696 & 9438337 & 3692972 & 302539 & 1032186 \\
\hline Totales & 30561459 & 1185546 & 4787930 & 11543858 & 5220994 & 508902 & 7287535 \\
\hline
\end{tabular}

Fuente: Marino y Zuleta (2010), a partir de http://biblioteca.itam.mx/recursos/ehm.html\# reforma (datos de Secretaría de la Reforma Agraria, Dirección General de Información y Documentación Agraria) 


\section{La cultura jurídica}

\section{Andrés Molina Enríquez}

En 1909, finalizando el porfiriato, Andrés Molina Enríquez ${ }^{8}$ publicó su gran obra, Los grandes problemas nacionales, libro de cabecera de los reformadores agrarios posteriores. Allí, consideraba al problema de la propiedad de la tierra como el más importante a resolver para garantizar el crecimiento de México. Sobre postulados positivistas e historicistas construyó un esquema evolutivo de las sociedades que entonces ocupaban el territorio mexicano. En este esquema el concepto de raza adquiere una importancia fundamental, ya que al origen étnico le adscribía, de manera muy determinista, no sólo rasgos físicos sino también características morales, de temperamento y de acción social.

La particular relación de un grupo con el territorio que ocupaba, es decir, su noción de dominio territorial, fue el elemento exclusivo que consideró para clasificar los cinco estadios evolutivos en los que se sucedían los distintos grupos o sociedades: el primero, el de las sociedades nómadas carentes de cualquier noción de derecho territorial; el último o superior, el de las sociedades de crédito territorial o de titulación fiduciaria. De este modo, en los cuatro primeros estadios ubicaba a los distintos grupos indígenas del territorio nacional, mientras que en el cuarto colocaba también a las ran-

8 Andrés Molina Enríquez (1868-1940), se tituló de abogado, ejerció como juez y notario en varios distritos rurales de su natal Estado de México, y se consideraba a sí mismo sociólogo. Se dio a conocer en 1905, al ganar un concurso de ensayo sobre el natalicio de Benito Juárez con la obra Juárez y la Reforma. Luego de su trabajo más famoso, Los grandes problemas nacionales(1909), abrió un despacho jurídico en la capital de la república con el Lic. Luis Cabrera. Destaca en el medio intelectual por su actitud crítica hacia los problemas evidentes del modelo económico y social desarrollado por el régimen del presidente Porfirio Díaz, y a partir de allí se vio envuelto en la política revolucionaria, específicamente en el reformismo agrario. En agosto de 1911 rompió con Francisco Madero y promulgó el Plan de Texcoco, en el que abogaba por una reforma radical que, conducida por el estado, expropiara y redistribuyera de una vez los latifundios (Molina Enríquez [1911] 458 y ss.). Esto provocó su encarcelamiento y fue causa de su fuerte polémica con Wistano Luis Orozco, quien defendía una reforma pacífica y de largo plazo. Gracias a su relación con Cabrera - rector de la Escuela Nacional de Jurisprudencia en 1912, hombre de confianza del presidente Venustiano Carranza y redactor de la Ley Agraria del 6 de enero de 1915 - y al prestigio adquirido por su obra, asesoró a los constituyentes de 1917 en la redacción del artículo 27 y ocupó diversos cargos públicos en los años 1920 y 1930. 
cherías de mestizos y consideraba que el quinto sólo había sido posible a partir de la conquista española. En su esquema evolucionista otorgaba un lugar importante a la desigual capacidad de los individuos al interior del grupo, donde la «selección» de «los más aptos» llevaba a fortunas desiguales y a una dispar posesión de terrenos.

La consecuencia más importante que derivaba de considerar cada estadio como escala ineludible en el proceso de desarrollo histórico de una sociedad, era su convicción de que el Estado podía coadyuvar acelerando dicho proceso evolutivo, pero que no tendría éxito una política que pretendiera hacerles saltar etapas de manera forzada. Así como la realidad nacional obligó a Molina Enríquez - y a muchos de sus contemporáneos, en México y América Latina - a matizar los fuertes postulados racistas de los autores que nutrían su argumentación - por ejemplo, en la caracterización del mestizo como el fundamento natural de la nacionalidad mexicana y el obvio dirigente de la marcha del país hacia el progreso -; esa misma realidad les llevó también a repensar el papel del Estado, separándose del laissez-faire de moda en los círculos políticos y académicos europeos, para demandar la intervención del Estado nacional en la corrección de la «defectuosa» realidad social y económica heredada de la colonia. La acción del Estado en determinadas áreas, a través de políticas correctamente diseñadas - como las que sugiere Molina Enríquez en su libro -, era vista como indispensable para lograr el cambio social y transformar a México en una nación moderna. En el campo de la propiedad, las soluciones propuestas por este autor van decididamente en ese sentido. ${ }^{9}$

Este libro de Molina Enríquez fue de enorme influencia en los reformadores agrarios, proceso en el que él mismo, aunque de manera colateral, participó en sus inicios, paralelamente a su carrera en la administración pública y las aulas universitarias. Como colofón de su trayectoria, Molina publicaría otra gran obra en los últimos años de su vida: Esbozo de la historia de los primeros diez años de la revolución agraria de México (de 1910 a 1920), publicada en cinco tomos entre 1932 y 1936, y donde expuso sus puntos de vista sobre la política agraria desarrollada en la primer década revolucionaria. Es interesante analizar el modelo teórico-metodológico sobre el que armó este último libro para definir los cambios operados en las ciencias sociales y el

9 Marino (2001).

166 Daniela Marino 
derecho en el decurso de la acción pública de Molina (1909-1936) y que es, grosso modo, la misma periodización de este artículo. En este sentido, en 1936 veía inconclusa la revolución, como

uno de tantos episodios (el más profundo y trascendente) de las luchas agrarias comenzadas desde la Independencia, para destruir los latifundios que son las raíces madres de la organización social por castas que subsiste todavía. ${ }^{\mathbf{1 0}}$

Seguía siendo manifiestamente positivista, y todavía en el prólogo al quinto tomo (1936) reconoció el valor de la obra de Auguste Comte, pero ya consideraba obsoletas las teorías evolucionistas

En el estado actual de las ciencias démicas, es fácil de comprender que no existe la línea invariable de la trayectoria obligada que se creía para todos los pueblos, como no hay tampoco al fin de esa línea, el punto de llegada de ella, que se llama civilización. No es por lo mismo exacta la apreciación que antes se había deducido del principio de la evolución, acerca de que las diferencias que presentan los pueblos, dependan de su adelanto o de su atraso en el curso de la trayectoria común. ${ }^{\mathbf{1 1}}$

En cambio, en esta obra apeló a una contraposición entre dos tipos de sociedades que definió como oriental y occidental, siendo la diferencia principal entre ambas que «en los pueblos de esa cultura (oriental) (no existe) el concepto de derecho, mucho menos el de derecho de propiedad, y menos aún, el de derecho de propiedad sobre las tierras y las aguas». ${ }^{\mathbf{1 2}}$ Siguiendo las teorías del poblamiento americano, caracterizó a los grupos indígenas como orientales, y a las sociedades americanas postcoloniales como un tipo intermedio entre ambos arquetipos. Nuevamente realizó un análisis histórico - con fuerte énfasis en la variable raza - aunque sólo para tener los elementos suficientes para alcanzar el conocimiento de la sociedad presente, que lograría a través de la Etnología. Consideraba entonces a la Historia sólo como ciencia auxiliar de ésta, pero por sí sola insuficiente - aún la historia marxista, interpretación que reseña en su obra - de allegar una comprensión integral de una sociedad determinada en un período limitado de tiempo.

También criticó el determinismo racista que sustentara en 1909, y ya no creía en un papel decisivo de los mestizos para el futuro de la nación mexicana. Reiteraba los juicios acerca de la propiedad de la tierra y de las leyes emitidas que elaborara en su libro anterior. Por ejemplo, respecto a que

10 Molina Enríquez (1985) 504.

11 Molina Enríquez (1985) 68-69, cursiva del autor.

12 Ibídem 39. 
las culturas indígenas no habían alcanzado los conceptos de derecho y de propiedad, y como tampoco tenían escritura se vieron imposibilitadas de comprender el sistema occidental de titulación de la propiedad. O bien, sobre las ventajas que tenía reconocer, al menos transitoriamente, el régimen comunal para los pueblos de indios; idea que retomó la primera Comisión Nacional Agraria. ${ }^{13}$

Específicamente, en el libro V: «El principio de la verdadera Revolución. Hechos que trataron de formular y de reducir a reformas concretas y positivas, los ideales revolucionarios de renovación social», analiza el proyecto ejidal de Luis Cabrera durante la presidencia de Madero y el artículo 27 de la Constitución de 1917. Respecto al primero, Molina Enríquez concordaba con Cabrera - sugiriendo que este último retomaba las ideas expuestas en Los grandes problemas nacionales-, en cuanto defendía la propiedad comunal ejidal inalienable pero sólo mientras no fuera posible crear un sistema de explotación agrícola en pequeña propiedad, que sustituyera a las grandes explotaciones de los latifundios como un complemento al salario del jornalero.

Esta reforma, sin dejar de ser liberal, fue sumamente innovadora respecto a la tradición que, gobierno tras gobierno, legislaba sobre la desamortización de las tierras comunales. Cabrera propuso devolver la personalidad jurídica a las instituciones municipales para poder adquirir, poseer y administrar bienes raíces. Se expropiarían tierras para reconstituir los ejidos, poniendo la propiedad en manos de la Federación y dejando el usufructo a los pueblos. El Congreso no hizo ley este proyecto, pero éste fue antecedente al decreto de 6 de enero de 1915 y al artículo 27 constitucional, en cuya redacción asesoró Andrés Molina Enríquez. El autor consideraba esta reforma como «revolucionaria» $\mathrm{y}$ «trascendente», ${ }^{\mathbf{1 4}}$ pero también, parcial e incompleta:

... el Lic. Luis Cabrera [se apartó] de la disolución de los latifundios por fraccionamiento en una copiosa pequeña propiedad, como lo aconsejábamos nosotros, para concentrarse en el interés inmediato de los pueblos, dando origen a la futura legislación sobre los ejidos.

[...] el problema agrario, por lo mismo, iba a dejar en pie las haciendas, y a reducirse como en la Época Colonial, a la restitución y a la dotación de ejidos a los pueblos. ${ }^{15}$

13 Ibídem 460-461.

14 Molina EnríQuez (1985) 462-463.

15 Ibídem 450 y 484. 
Al referirse a su participación en el Constituyente de Querétaro, volvió a hacer hincapié en este punto, insistiendo sobre el carácter limitado y parcial de lo que él llamaba la «solución ejidal», como aparecía en el proyecto del artículo 27 redactado por la presidencia, mientras que él habría indicado al Ministro de Fomento cómo resolver el problema agrario «de manera integral». ${ }^{\mathbf{1 6}} \mathrm{El}$ artículo fue redactado de otra forma a la sugerida por Molina Enríquez, pero me interesa señalar cómo nuestro autor insistió en sus ideas sobre que la reforma agraria debía pasar ineludiblemente por la disolución de los latifundios; que tan importante como la tierra misma eran los derechos sobre aguas, pastos y otros recursos; y que al reconocer derechos de propiedad amplios no solamente títulos escritos - estaba reconociendo, entre otros, los derechos de comunidades y pueblos. Para lograrlo, nuevamente apelaba, como única solución, al poder necesario del Estado para sobreponerse a los intereses particulares y romper la inviolabilidad de la propiedad privada que impedía disolver los latifundios, de manera que

pudieran estar los Poderes Públicos representantes de nuestra Soberanía, en condiciones de regular, de limitar y aun de agotar y de destruir, los derechos de la propiedad privada, que quisieran por perversidad o por egoísmo, superponer dichos derechos a los intereses colectivos de la comunidad.

El objetivo era crear una estructura mayoritaria de pequeños propietarios para lograr: 1- aumentar la producción agrícola de modo de alcanzar el abastecimiento nacional, 2- abaratar esa producción lo más posible, 3- que la producción se hiciera en pequeñas parcelas para distribuir los beneficios entre el mayor número posible de familias y 4- que dicha producción respetara la diversidad étnica y cultural de los grupos sociales, de modo de impulsar el crecimiento y desarrollo de la población en su conjunto «ya entonces convertida en una verdadera nacionalidad». ${ }^{\mathbf{1 7}}$

Molina Enríquez resaltaba, en principio, la victoria conseguida al imponer constitucionalmente el derecho preeminente del Estado sobre los particulares en materia de tierras, aguas y elementos del subsuelo. Pero, en segundo lugar, insistía en el modelo ideal de la pequeña y mediana propiedad, muy vinculado al proyecto de nación moderna y mestiza. Si rescataba, entre los demás apartados del artículo 27 ,

16 Ibídem 492, cursiva del autor.

17 Molina Enríquez (1985) 498-499. 
el que devolvió a todas las agrupaciones de población que de hecho o por derecho guardan el estado comunal, la capacidad legal para tener tierras y aguas: el que declaró la nulidad de todas las diligencias, disposiciones, resoluciones y operaciones que hubiesen privado a los pueblos de sus tierras y aguas, mandando restituírselas o dotarlos con tierras y aguas nuevas, de modo que siempre tuvieran las que pudieran necesitar. ${ }^{18}$

era porque, como en Los grandes problemas ..., aceptaba este principio pero de ninguna manera lo consideraba un avance en el desarrollo de la sociedad mexicana. Por el contrario, luego de reconocer que los grupos indígenas e «indio-mestizos» se inclinaban por «idiosincracia» al sistema de propiedad comunal, concluía que el Congreso Constituyente de Querétaro debía seguir siendo individualista y liberal, y no apartarse de la cultura occidental, a la que, más allá de sus orígenes, debía pertenecer la nación mexicana. ${ }^{19}$

El cambio en su postura radica en que en el libro publicado en 1909 consentía en la permanencia de la propiedad comunal como un estadio en el camino evolutivo que inexorablemente los pueblos indígenas debían recorrer hasta llegar al estadio más avanzado, de acuerdo a su adopción de las teorías darwinistas y evolucionistas. En 1936 ya no comulgaba con éstas, pero sin embargo apoyaba el programa de restitución ejidal diseñado por el gobierno, no ya desde un punto de vista teórico sino meramente instrumental: la aceptaba como la solución al problema de la tierra posible de realizar en ese momento político; no la deseable, que para él seguía siendo la disolución de los latifundios $y$ de las comunidades en aras de constituir un sistema de multitud de pequeñas y medianas propiedades.

En suma, concluyo que Molina Enríquez nunca dejó de enarbolar una visión liberal del problema de la tierra - si bien crítica de la desamortización liberal decimonónica que desposeyó a los indígenas; y diferente de liberales como Orozco, quien también consideraba improductivos los latifundios pero arguía que era el mercado y no el Estado quien debía, lenta y pacíficamente, subdividirlos -, aún después de veinticinco años del estallido de la revolución. No obstante, es de notar ciertos cambios en las ideas de Andrés Molina Enríquez, motivados tanto por su participación en el gobierno como por la evolución teórica en las ciencias sociales y en el derecho mismo. Así, hemos visto que si bien a mediados de los años 1930 seguía siendo positivista, ya no comulgaba con la concepción evolucionista de la sociedad y, por otra parte,

18 Ibídem 502.

19 Ibídem 497.

170 Daniela Marino 
defendía cada vez más la acción decidida del Estado en la economía, para tutelar los derechos de los actores sociales más débiles y para garantizar los intereses comunes por sobre los individuales, al punto de definir el sistema de propiedad establecido por el artículo 27 de colectivista, estableciendo categóricamente el origen social de la propiedad y el derecho preeminente de la Nación sobre su territorio, por encima del derecho de los particulares.

\section{Los abogados y la Revolución}

Probablemente fue el liberalismo de Molina Enríquez, y de Cabrera, el que motivó que dichos personajes no siguieran siendo influyentes en los años 1930, así como que el Esbozo ... de Molina Enríquez no adquiriera el influjo que continuó teniendo Los grandes problemas. El liberalismo de Cabrera se manifestó de manera patente en la redacción de la Ley Agraria de 6 de enero de 1915 y fue coincidente con el proyecto constitucional que presentó el presidente Carranza al Constituyente de 1916-17: en el plano agrario, ambos planteaban, al igual que Molina, las ventajas de la pequeña propiedad y para ambos la expropiación de los grandes latifundios y su reparto a los pueblos significaba medidas intermedias hacia aquel fin pero, de ninguna manera, pretendían la reconstitución de las antiguas comunidades de indios. Para ello, otorgaron la atribución de la expropiación a la autoridad administrativa, pero dejaron abierto el recurso judicial para fijar el valor de la propiedad y para que los particulares afectados pudieran deducir sus derechos.

Pero Cabrera no sólo manifestaba la cultura jurídica de su época, sino que incluso su participación política resultó chocante en la Escuela Nacional de Jurisprudencia - integrada a la recientemente refundada Universidad Nacional en 1910 -, a la que llegó como director en 1912, durante la presidencia de Francisco Madero. Los profesores de la escuela eran notorios abogados porfiristas, al igual que muchos de los estudiantes de derecho, quienes reaccionaron ante una serie de medidas disciplinares que pretendió tomar Cabrera yéndose a la huelga y, finalmente, abandonando la Universidad para fundar la Escuela Libre de Derecho. ${ }^{20}$ La crisis en el ambiente jurídico, producto de la supervivencia de una élite de abogados liberales positivistas que dominaban el foro y la enseñanza, en un ambiente político que tomaba

Del Arenal (2010), cf. Garciadiego (1996). 
iniciativas sociales, iba a perdurar hasta los años de 1930. En palabras de un futuro gran abogado que ingresó a la Escuela Nacional en 1915, Lucio Mendieta y Núñez:

Durante el período de 1915 a 1920, se inicia, en la Escuela de Leyes, aun cuando lentamente, una honda transformación. Todavía durante esa época, el profesorado se encuentra constituido, en su mayoría por abogados de gran prestigio; pero pertenecientes, por su extracción social, por su educación, por sus ideas, al antiguo régimen político. La juventud que se iniciaba en la carrera del Derecho, se encontró así con una constante contradicción entre la Escuela que en nombre de viejos principios de Derecho condenaba la revolución y cuanto de ella procedía, o que la ignoraba olímpicamente, y la vida palpitante de México, que ofrecía en sus movimientos revolucionarios un gran contenido social.

Entre los profesores de entonces, recordamos al licenciado Victoriano Pimentel en la cátedra de Derecho Civil, puntual, estricto, metódico [...] en una constante aplicación a los mandamientos de nuestro Código Civil de 1884 [...] Manuel Mateos Alarcón, anciano venerable, pulcro, puntualísimo en sus clases, hablaba rápidamente con su voz senil, citando de memoria los artículos del Código Civil [....$^{\mathbf{2 1}}$

Recientemente, un autor ha revisado puntualmente el discurso agrario del último lustro decimonónico y las tres primeras décadas del siglo $\mathrm{XX}$ y encuentra igualmente la continuidad del liberalismo hasta el debate constituyente de 1916-17. De hecho, cita un ejemplo apenas anterior a dicho congreso, de un autor que utiliza el concepto «función social» refiriéndose a la propiedad. ${ }^{22}$

\section{La Constitución de 1917}

Los trabajos del Congreso Constituyente de 1916-17 han sido generalmente estudiados como reflejo de la coexistencia de ambas concepciones del derecho vigentes en la cultura jurídica de la época: liberal y social. Así, se ha interpretado el texto constitucional como uno básicamente liberal en el que se insertaron unos pocos pero innovadores artículos en muy específicas materias: el 3 (educación), el 27 (propiedad), el 5 y el 123 (trabajo). En esta

21 Mendieta y NúÑEZ (2002) 23.

22 Se trata de uno de los personajes públicos del reformismo agrario, Fernández González Roa, quien en 1916 y citando explícitamente una ley inglesa, refiere que «algunas legislaciones han establecido ya la forma en que debe concederse el derecho de propiedad, que ha cesado de ser un dominio absoluto para convertirse en una función social», cit. en: BARRón (2010) 142. 
percepción, se identifica al grupo liberal como aquél más cercano al presidente Carranza, el que defendió el Proyecto de Reformas presentado por éste, y que veía a la revolución "como un proceso político que concluía con la sustitución de autoridades y la corrección de ciertos y definidos excesos o carencias de libertades», y a la Constitución como reflejo de la realidad y declarativa de derechos; enfrentado a otro grupo que, sobre todo a partir de la discusión del artículo 5, referido a los derechos y límites asociados a la garantía de libertad de trabajo, «identificaban a la revolución como reivindicatoria y, por ende, exigían un cambio en el modo de articular las relaciones sociales en general», y veían a la Constitución como producto de una lucha política y social que exigía transformar el estado de cosas, no sólo cambiando la élite en el poder sino también las mismas condiciones de dominación, de modo de incorporar a la constitución las demandas de obreros y campesinos que habían hecho la revolución. ${ }^{23}$

Esta última habría sido la que triunfó, logrando así la posibilidad de proponer un título completo sobre materia obrera - título sexto «Del trabajo y la previsión social» añadiendo al artículo 5 el $123 .{ }^{24}$ Esta misma discusión resurgió al discutir el artículo 27 sobre propiedad, mismo que en la constitución de 1857 había impuesto el modelo de propiedad privada, individual y perfecta, aboliendo la propiedad corporativa y amortizada que ahora - en 1917 - se buscaba restaurar, aunque bajo un modelo distinto al colonial. Así, permanecía la propiedad privada reconocida como garantía individual, al tiempo que reconocían que la tierra estaba distribuida muy desigualmente, lo que causaba perjuicios al desarrollo nacional, al autoabastecimiento alimentario y a las condiciones de vida de los jornaleros, de modo que la Constitución debía imponer límites a esa garantía individual garantizando, por medio de la expropiación y reparto de los latifundios, una distribución más equitativa del suelo agrícola. Así, el artículo 27 estableció que la propiedad del suelo y el subsuelo corresponden originariamente a la Nación, derivando de ello que el Estado puede imponer a la propiedad privada las modalidades que dicte el interés público; es decir, que los derechos sociales están por encima de los derechos particulares.

Por el contrario, otros autores no ven dos grupos en pugna en el constituyente, sino más bien distintos modelos estatales - distintas tradicio-

23 Cossío Díaz (1998) 197-198 y 201-205.

24 Ibídem 198-199. 
nes jurídicas - conviviendo en el texto constitucional. Para González y Caballero, por ejemplo, la carta de 1917 recoge elementos del liberalismo decimonónico, tomados directamente de la constitución de 1857 que se pretendía reformar; elementos centralistas, ya presentes en reformas anteriores a dicha constitución y que habían permitido el ejercicio autoritario del presidente Porfirio Díaz; y elementos sociales que fueron los más novedosos y daban cuenta de los cambios que experimentaba entonces la ciencia jurídica y constitucional. ${ }^{25}$ Por ejemplo, la tesis de licenciatura en derecho defendida en 1919 por Vicente Lombardo Toledano - compañero de estudios del citado Mendieta y Núñez -, daba cuenta ya, en su primer capítulo, de las nuevas teorías filosóficas, entre las que reseñaba el materialismo de Marx y Engels y, en el capítulo dos, «El concepto moderno del Estado», ya iniciaba con un epígrafe de León Duguit, autor que reseña ampliamente en tal capítulo. Pasa luego a referir las transformaciones del derecho internacional a consecuencia de la primera guerra, para finalizar apuntando críticamente las innovaciones que la constitución de 1917 introdujo en el derecho mexicano, y es particularmente duro con las consecuencias que estima producirá el art. 123, punto que no deja de sorprender en el pensamiento del futuro sindicalista marxista. ${ }^{26}$

\section{La polémica de 1925 , ¿qué es un derecho revolucionario?}

En 1925 resultó electo Plutarco Elías Calles, primer presidente que no había destacado militarmente en el movimiento revolucionario y, por lo mismo, necesitado de legitimidad. Calles ejerció un periodo presidencial de cuatro años, pero fue la figura fuerte detrás de los tres presidentes siguientes (periodo conocido como «maximato»), hasta que el presidente Lázaro Cárdenas, quien asume en 1934, lo obliga a exiliarse. La manera de obtener legitimidad, para Calles, se reflejó en lo que Díaz Arciniega llamó «querella por la cultura 〈revolucionaria»", y que él analiza en los ámbitos de la cultura y del derecho. Esta querella tenía sentido porque todavía quedaban elementos activos del antiguo régimen porfirista - y muy notorios en el campo jurídico -, a la par que se consolidaban los grupos revolucionarios. El principio unificador que analiza este autor es la «lealtad revolucionaria», que no era más que el control

25 González, Caballero (2002) 49-51.

26 Lombardo Toledano (1919). 
de las expresiones políticas bajo una ideología nacionalista y popular, sin romper totalmente con el viejo molde liberal. ${ }^{27}$

El debate al que hace referencia se produjo en enero de 1925, otra vez a causa del nombramiento de un nuevo director de la Facultad de Jurisprudencia. Pero a diferencia de Luis Cabrera en 1912, ahora Aquiles Elorduy invitó a regresar a la universidad a algunos viejos profesores positivistas. A este hecho le sigue un debate que dura seis meses en la prensa, en el que algunos opinan que es positivo el regreso de profesores con experiencia, mientras otros se escandalizan por la llegada de maestros «reaccionarios», «de prestigio rancio y oropelesco», cuando preferirían maestros jóvenes vinculados a las nuevas teorías del derecho, «de pensamiento revolucionario» y «en los senderos del socialismo». ${ }^{\mathbf{2 8}}$ A fines de marzo es cesado el Lic. Eduardo Pallares de su cátedra, por una nota periodística que había publicado y que denotaba «deslealtad revolucionaria» (Pallares fue, desde la prensa, uno de los críticos más duros de Calles). Varios periódicos se enfrascan en el debate en torno a temas como la libertad de prensa, de cátedra y de pensamiento, la autonomía universitaria o el derecho del Estado a reclamar una línea ideológica de los maestros que paga el presupuesto.

Podemos notar en la prensa que todavía es dominante el uso de las diversas concepciones del derecho como elemento disciplinador de las élites, para separar a los que están con el gobierno de quienes manifiestan oposición a las políticas dictadas por el callismo. Sin embargo, la «socialización del derecho» es materia conocida y de discusión, aunque no sea aún un rasgo generalizado de la cultura de los operadores del derecho, mas tampoco de las políticas públicas. ${ }^{29}$ De hecho, durante su presidencia Calles pretendió dar

27 Díaz Arciniega (1989) 180-181.

28 Ibídem 200-201.

29 Por ejemplo, Narciso Bassols en «El pensamiento de la gente nueva» (El Universal, 8-5-1925), intenta discernir el socialismo actual de las viejas doctrinas utópicas, de Marx y Proudhon e incluso de la revolución rusa. Hilario Medina en «Un nuevo derecho» (El Universal, junio 1925), estima que vivían entonces «en plena revolución de ideas [...] el preludio de las nuevas fórmulas del derecho público y del derecho privado [...] la familia, la propiedad y el Estado son cosas enteramente diferentes de lo que fueron en la pasada generación»; mismas que Eduardo Pallares, en cambio, tilda de «Sangrientas utopías» (El Universal, 12-5-1925), pues de creer a estos autores, «la humanidad pensadora se ha vuelto rabiosamente socialista», y para Nemesio García Naranjo son «Amalgamas ilusorias » (El Universal, 16-5-1925), pues «eso que ha dado en llamarse spensamiento nuevo> [...] sólo es una sumisión humilde a los cánones políticos imperantes». 
por terminado el reparto agrario, e incluso la Corte Suprema de este periodo pone fin a su política posterior a 1917, de negar los amparos a los grandes propietarios que entorpecían las dotaciones agrarias, para establecer en cambio una nueva política de amparar las garantías individuales de quienes se decían afectados por las acciones agrarias. Esta nueva política durará hasta las reformas constitucionales de 1928 y $1931 .^{30}$

Con una Suprema Corte disciplinada, con la sistematización de la legislación de la materia agraria, posible por la elaboración del Código Agrario aprobado por el Congreso en marzo de 1934, pocos meses antes de que Lázaro Cárdenas asumiera como nuevo presidente de la República, y con el convencimiento de éste acerca de las responsabilidades sociales del Estado, la década de 1930 se percibe como el periodo en que, sin obviar la continuación de divisiones en el campo jurídico, se estableció un mayor consenso - al menos hasta 1937 - y divulgación en la enseñanza y la operación del derecho, de lo que entonces se llamaba, siguiendo a Duguit, «la socialización en el derecho». ${ }^{31}$

Retomando nuestras preguntas iniciales, podemos concluir, provisoriamente, señalando que la Revolución efectivamente forzó medidas de retribución social a grandes grupos de población, en particular campesinos, que habían participado en la fase armada del movimiento. Sin embargo, el cambio de la estructura agraria ni fue enteramente una innovación posterior al porfirismo, ni tampoco fue fácil establecer el consenso respecto de su necesidad, oportunidad y, fundamentalmente, su base ideológica y los mecanismos concretos del reparto. En este sentido, tomó más de una década formar una nueva generación de juristas y abogados que supliera el antiguo

30 Barrón (2010) 153-160. Ver también "Así se dijo en la Cámara de Diputados», El Universal, 19-5-1928: las reformas al art. 32 constitucional suprimen la inamovilidad judicial, aumenta a 16 el número de ministros, obliga a trabajar en salas cuando trate asuntos secundarios y en pleno para los negocios trascendentales, el Presidente nombrará a los ministros; y «La nueva organización de la Corte», El Universal, 31-5-1928, «se ha propuesto con frecuencia mutilar todavía más el amparo». También «Magistrados de 25 años» en Excelsior, 25-7-1928, «La renovación de la Suprema Corte» en El Universal, 15-10-1928; «Revolucionarios en la Suprema Corte. Magistrados adictos a la Constitución», El Universal, 20-10-1928: «una mentalidad jurídica vinculada con la ideología del momento [...] los nuevos ministros deben ante todo estar penetrados de los verdaderos móviles que inspiran la Constitución».

31 Olea y Leyva (1933). 
personal liberal-positivista y ocupara posiciones en la cátedra, la cámara de diputados, los tribunales y la Suprema Corte para hacer realidad los elevados principios sociales de la revolución, plasmados en la constitución de 1917.

\section{Bibliografía}

BARRón, Luis (2010), La ‘modernización` revolucionaria del discurso político liberal: el problema agrario entre 1895 y 1929, en: Marván Laborde, Ignacio, La Revolución mexicana, 1908-1932, México: CIDE - FCE, 102-165

Cossío Díaz, José Ramón (1988), Las concepciones del derecho en el Constituyente de 1916-1917, en: Anuario Mexicano de Historia del Derecho X, 193-205

Cuadros Caldas, Julio (1999), Catecismo agrario, reedición de la sexta (1932), con un estudio introductorio de Guillermo Palacios, México: CIESAS

Del Arenal, Jaime (2010), La enseñanza del derecho durante los años de la Revolución, en: 20/10 Memoria de las Revoluciones en México, tomo 8, México

Díaz Arciniega, Víctor (1989), La querella por la cultura «revolucionaria〉 (1925), México: Fondo de Cultura Económica

Fabila, Manuel (1981), Cinco siglos de legislación agraria, 1493-1940, México: SRACEHAM

Garciadiego Dantan, Javier (1996), Rudos contra científico: la Universidad Nacional durante la revolución mexicana, México: El Colegio de México

García Ugarte, Martha (1995), La propuesta agraria de Venustiano Carranza y los Sonorenses (1915-1929), en: Estudios. Filosofía - historia - letras no 41, México: ITAM

González, Ma. del Refugio, José Antonio Caballero (2002), El proceso de formación del Estado en México. Los modelos de Estado en la Constitución de 1917, en: Serna, José Ma., José A. Caballero (eds.), Estado de derecho y transición jurídica, México: IIJ UNAM, 47-93

Knight, Alan (1986), The Mexican Revolution, 2 vols., Cambridge

Lira, Andrés (2009), Los derechos del hombre, las personas morales y el juicio de amparo en los albores del siglo XX, en: Del Arenal, Jaime, Elisa Speckman (coord.), El mundo del Derecho. Aproximaciones a la cultura jurídica mexicana (siglos XIX-XX), México: Ed. Porrúa - Instituto de Investigaciones Históricas UNAM - Escuela Libre de Derecho, 111-171

Lombardo Toledano, Vicente (1994), El derecho público y las nuevas corrientes filosóficas [1919], tesis de licenciatura, Escuela Nacional de Jurisprudencia, impresa en: Obras, tomo I, vol. 1, México: CEFPyS Vicente Lombardo Toledano, 51-109

Mannori, Luca, Bernardo Sordi (2004), Justicia y administración, en: Fioravanti, Maurizio (ed), El Estado moderno en Europa. Instituciones y derecho, Madrid: Trotta, 65-102 
Marino, Daniela (2001), El problema de la tierra y la propiedad comunal indígena en Andrés Molina Enríquez. Antes y después de la Revolución, en: IZTAPALAPA. Revista de Ciencias Sociales y Humanidades 51, julio-diciembre, 205-224

Marino, Daniela, Ma. Cecilia Zuleta (2010), Una visión del campo. Tierra, propiedad y tendencias de la producción, 18530-1930, en: Kuntz, SANDra (coord.), Historia económica general de México, México: El Colegio de México - Secretaría de Economía, 437-472

Mendieta y Núñez, Lucio (2002), Apuntes para la historia de la Facultad de Derecho [1939], México: UNAM

Molina EnRíQueZ, ANDrÉs (1978), Los grandes problemas nacionales [1909], México: Era

Molina Enríquez, Andrés (1985), Esbozo de la historia de los primeros diez años de la revolución agraria de México (de 1910 a 1920), México: CFE - INEHRM ( $1^{\circ}$ ed: $\left.1932-1936\right)$

Olea y Leyva, Teófilo (1933), La socialización en el derecho. Ensayo de una teoría general de las funciones, México: Editorial El Hecho mexicano

Orozco, Wistano Luis (1895), Legislación y jurisprudencia sobre terrenos baldíos, 2 vol., México: El Tiempo

Velazquez, Erick et al. (2010), Nueva Historia de México, El Colegio de México 


\section{El proyecto de modernización del ideario liberal republicano en Brasil en cuestión: las ediciones de la «Consolidação das leis civis» durante la «República Velha» (1889-1930)*}

\section{Introducción}

Aunque se diga que hubo una tradición liberal desde la independencia brasileña, pues el país se organizó como monarquía constitucional en la Constitución de 1824, no se puede negar que, a partir de la década de 1870 , se afirmó la necesidad de la adopción en Brasil de modelos jurídicos liberalrepublicanos en boga en Europa, resultando de al menos dos factores: la circulación de ideas políticas y jurídicas europeas, entendidas en el contexto como modernizadoras (desde la perspectiva de quienes detenían el poder en el inicio de la República), en élites de la época (militar, académica, política); y la repercusión del más grave conflicto bélico del período, la Guerra del Paraguay (1864-1870), con que se ha introducido un nuevo conjunto de ideas que se vinculaban a ese republicanismo. En esa senda, ese texto intentará reflejar un aspecto que se puede decir paradojal del período, en que se percibe un tipo de paralelismo entre el discurso liberal modernizador y la práctica jurídica tradicional.

De hecho, las fuerzas políticas en el comienzo de la República en Brasil tenían y mucho relación con el sistema anterior del Imperio. Y con esas fuerzas, los respectivos discursos, tanto del liberalismo más radical, como de grupos más conservadores; para ello, basta con recordar la presencia notoria de políticos imperiales (como el Barón de Rio Branco, renombrado diplo-

* Texto ahora consolidado de la ponencia presentada en el Seminario «Derecho privado y modernización en América Latina y Europa en la primera mitad del siglo XX», que se impartió en la sede del Instituto de Investigaciones de Historia del Derecho (Buenos Aires, Argentina), entre los días 4 y 6 de julio de 2012, organizado por el mismo Instituto y por el Max-Planck-Institut für europäische Rechtsgeschichte. 
mata de la época) en los primeros decenios republicanos. La cuestión que surge es la siguiente: ¿hubo una adhesión completa al discurso liberalrepublicano en los varios extractos de la sociedad brasileña luego de la proclamación de la República? La reflexión a seguir intenta presentar un cuestionamiento concreto de la pretendida convicción y conciencia social en favor del liberalismo republicano en sus orígenes, pero sin la intención de agotar el debate, pues se parte de una constatación de la práctica jurídica de la época, con sus vicisitudes. ${ }^{1}$

\section{Presentación del problema}

1.1 El contexto del debate sobre la modernización jurídica en Brasil de fines del siglo XIX a comienzos del siglo XX

Para efectos de presentación del tema, se debe recordar que el proceso histórico-político en Brasil en el siglo XIX, desde la independencia en 1822 ante Portugal hasta la República en 1889 , se mostró singular por sus características que difieren de lo ocurrido en otros parajes, aunque se hable de un proceso de independencia política y al fin y al cabo en proclamación de una República. ${ }^{2}$

1 Importa recordar que las fuerzas políticas que tenían su base en el juego político posterior a la Guerra de Paraguay se han adecuado obviamente a los parámetros de la República. Así, según recuerda Christian Lynch: «à sombra da mesma ideologia democrática e modernizadora do ralo republicanismo urbano, surgiu um vigoroso republicanismo do campo, amparado ideologicamente no darwinismo social, oligárquico e escravocrata, potencialmente autoritário, que sedutoramente acenava à aristocracia rural como a solução definitiva de seus principais problemas políticos. Posta em xeque pelo fracasso do parlamentarismo aristocrático da Lei Saraiva e pelo avanço do abolicionismo, a nova proposta da reação aristocrática contemplava uma república despida uma instância autônoma e suprema de representação do conjunto da população e que poderia ser mobilizada para impor uma agenda própria, que eventualmente beneficiasse os excluídos; uma república, em síntese, onde o Estado estivesse atrelado exclusivamente aos interesses das elites regionais. $\mathrm{O}$ discurso republicanismo se credenciou então como a nova linguagem por excelência da reação aristocrática, que como tal daria o golpe de morte na monarquia 15 de novembro de 1889 . Penso que essa dualidade entre republicanismo urbano e rural deverá explicar a hegemonia conservadora durante o regime e a decepção dos setores urbanos com esta república, que não era, como se dizia então, a dos seus sonhos.» - LYNCH (2010) 13.

2 Según habría de afirmar Christian Lynch: «Nenhuma das constituições brasileiras foi cercada de tantas expectativas e considerações como aquela que primeiro serviu de marco legal à República. Quando, no fim do Império, os republicanos democratas volviam os olhos para a 
A pesar de eso, en Brasil el proceso histórico-político ocurrió de una forma particular en razón de la cultura lusitana que sirvió como telón de fondo, como también de las personas involucradas en las disputas por el poder y por las ideas que se presentaban como modelos de las directrices políticas e históricas. Eso es determinante para que se pueda establecer una comprensión del panorama de los hechos posteriores en la edad republicana, a partir de la instauración de la República en Brasil en 15 de noviembre de 1889.

Desde esa perspectiva, la importación de ideas y modelos, sobre todo europeos, para el Brasil que estaba construyendo su historia se sucedía dentro de un dilema que siempre existió en el imaginario brasileño - la ambigua cuestión de la continuidad o no de la historia, cultura y sociedad de Portugal. ${ }^{3}$ En el caso de Brasil, lo que se percibe es un proceso de independencia intelectual y cultural con relación a Portugal durante el siglo XIX que, a su vez, se recrudece en el inicio del siglo XX después de instaurada la República. De ahí que se pueda decir que tal hecho político representa intachablemente,

vizinha Argentina, ficavam extasiados com o seu crescimento econômico e o atribuíam ao seu modelo constitucional, elaborado à imagem e semelhança dos Estados Unidos. Para eles, a Constituição do Império continha um vício de origem: o fato de ter sido outorgada por Pedro I depois da dissolução da Constituinte. Agora tudo seria diferente. Derrocada a monarquia unitária que supostamente entravava o progresso e adotada a república federativa, legitimada por uma Constituição elaborada pelos representantes do povo, o País seria refundado; tudo seria diferente. Para esses entusiastas, a Constituição de 24 de fevereiro de 1891 (pois era assim que ela era conhecida) preparava o País para uma era de verdadeira democracia, grandeza e prosperidade, que nos associava definitivamente ao movimento do continente americano» - LYNCH (2011) 298.

3 En ese caso, dicho legado portugués se manifestaba como legado colonial, de ahí el problema. Con todo, esa influencia europea siguió siendo el patrón del pensamiento de las clases dirigentes tanto en el Imperio como en la República, solamente que con la diferencia que no se trata de seguir a esa imagen del Portugal que subyugó a Brasil, sino seguir a lo último de las naciones de referencia en progreso y modernidad, es decir Francia, Inglaterra y Alemania, cada una según necesidades típicas (los modelos de codificación, o constitución, o de ciencia, por ejemplo). Es relevante retener lo que dice Nelson Saldanha: «o movimento codificador nas diversas nações latino-americanas, que como no velho mundo se seguiu ao essor das Constituições escritas, revelou em diversos países figuras notáveis de juristas, em cujas obras tem sido possível encontrar a marca de leituras características. Não tínhamos ainda - se se permite uma rápida digressão sociológica - uma «sociedade civil como a então constituída pela burguesia nas principais nações européias; mas as elites, nos países latino-americanos, formavam sua cultura nas fontes européias, de modo que os juristas do meado do século XIX, nestes países, eram (e não podiam deixar de ser) juristas europeizados» - SALDANHA (1985) 244-245. 
en el plano político, la existencia de un alejamiento continuo con referencia a la historia portuguesa en nombre de la construcción de una identidad brasileña, lo que sirvió para la política legislativa republicana de Brasil en los primeros tiempos. Y además, por lo que se puede juzgar del modelo constitucional que va a consolidarse en 1891, sucede el predominio de una visión oligárquica, que se consolida en la Política do café com leite, entre los Estados de San Pablo y Minas Gerais, respectivamente, del centro del país. ${ }^{4}$ Es relevante sopesar que el discurso liberal-republicano buscaba la adopción del modelo político de los Estados Unidos, pero la práctica resultó ser oligárquica, centrada en élites regionales fortalecidas con la autonomía de los Estados federados.

Fundamental en ese sentido sería la confirmación de la autonomía legislativa del país mediante la consecución de un proceso de codificación civil, el cual comenzaría aún durante el Imperio, a mediados del siglo XIX, hasta llegar a la publicación en $1^{\text {er }}$ de enero de 1916 del Código civil. Existe otro transcorrido posterior con los intentos de modificación o sustitución de ese texto legal hasta la publicación de un nuevo Código civil en 2002. Pero no es el tema ahora.

\subsection{La construcción del imaginario republicano desde el discurso de modernización jurídico-política}

Se puede aseverar que el proceso de independencia ante Portugal solamente habría de confirmarse mediante la consolidación de una autonomía legislativa en Brasil, tema ese que fue corriente desde mediados del siglo XIX. Pero los prejuicios en contra del Imperio se multiplicaban, pues los elementos sociales ya estaban cambiando, con repercusiones en las leyes (v. g. la abolición de la esclavitud en Brasil en 1888).

4 Afirma Lynch: «contrariando a expectativa dos liberais que haviam participado do processo contituinte, o exercício do poder político da Primeira República foi marcado pelo autoritarismo que sucessivamente lhe imprimiram as forças que a instauraram - o Exército e a aristocracia rural: primeiro, na forma de um militarismo positivista; depois, pelo conservadorismo oligárquico. Estabilizado depois de 1898, o regime republicano se acomodou como um arranjo das oligarquias estaduais coordenado pelo Presidente da República - a Política dos Governadores - cujo objetivo era o e impedir qualquer forma organizada de pluralismo político e, por conseguinte, preservar os vinte situacionismos da federação» - LYNCH (2011) 298. 
En la afirmación de la llamada República Velha, que en Brasil comprende el período 1889-1930, se puede apuntar que representó la victoria de un discurso liberal radical anterior que buscaba la caída de la Monarquía brasileña desde la década del 1870 . En ese panorama, algunos autores representaron un cambio en la visión teórica del Derecho, aunque no todos tuviesen pretensiones de defender un discurso político republicano (algunos de ellos más se presentaron como la superación de lo antiguo); a más de eso, otros autores declaradamente hacen alusión a discursos legitimadores de la República (a veces como negación de la Monarquía y la Iglesia Católica), defendiendo la propagación del ideario republicano de Norteamérica de un lado, y, a su vez, había los que iban a criticar duramente su época mediante la afirmación del darwinismo social al estilo de H. Spencer, por ejemplo, lo que se representaría la superación del Imperio.

Ejemplo de esa visión se presentaba más claramente en los autores de la Escuela de Recife, sobre todo a partir del pensamiento de Tobias Barreto y Sílvio Romero. Ese ideario, aunque no se diga que se suceda de modo uniforme, intenta mostrarse como la adecuación a un sentido de modernidad, el de acompañar y hacer variaciones respecto del debate europeo que se desarrollaba en ese período. Fue la base del discurso político y filosófico que decretó el final del Imperio en favor de una modernización en Brasil desde parámetros de la época, el positivismo y el evolucionismo, sustratos ideológicos que fueron defendidos por autores de tal Escuela. ${ }^{5}$

\subsection{Sobre el problema de la continuidad de la tradición jurídica lusobrasileña}

Sin embargo, el problema de la elaboración de un proyecto legislativo nuevo, en particular en el área civil, no podría dejar de pasar por los autores modernos. Así, no por acaso, un autor de la Escuela de Recife, Clovis Bevilaqua, recibe la incumbencia de hacer un proyecto de Código civil, el que es elaborado durante el año de 1899. De tal proyecto, que siguió los trámites típicos de un texto de esa relevancia, salió el Código civil que vendría a ser publicado en 1916.

5 Así, por ejemplo, en las palabras de Mario Losano: «In questa atmosfera, la modernizzazione culturale del Brasile inizia con la recezione e la discussione critica di due grandi movimenti della cultura europea: il positivismo e l'evoluzionismo» - Losano (1974) 337. 
La tramitación fue acompañada por los dirigentes políticos, las clases del ámbito jurídico y la población, de una manera general, demostrando así el consenso tácito que existía cuanto a la elaboración de un Código civil. Eso porque representaba el anhelo de la clase dirigente republicana de la adopción de un modelo moderno de Estado (siempre teniendo en mente el ejemplo norteamericano) y de Sociedad.

Entre tanto, aquí surgiría la cuestión tema de la presente investigación, partiendo de constataciones de la propia práctica jurídica de esa época: ¿por qué razón, dentro de ese espíritu nacional-republicano de matiz liberal, un libro publicado en mediados del siglo XIX, alterado en dos ediciones todavía durante el Imperio, hecho por un autor que fue considerado como loco en el final de su vida y que notoriamente defendió en vida la visión de continuidad de la tradición jurídica lusobrasileña en contra de modismos de época, tendría lugar en el mercado editorial brasileño por veinte y cinco años durante la República? Es decir: ¡cómo se explica una reimpresión de la tercera edición de ese libro en 1896; y después otras dos ediciones más, una en 1910 en el medio del debate en el Congreso y la otra en 1915, a un año de la publicación del Código?

Se habla aquí de la Consolidação das leis civis de Augusto Teixeira de Freitas, el texto que ha tenido por objetivo ser la recopilación de toda la legislación civil vigente en Brasil durante el Imperio. Y aquí se encuentra el punto central de esa investigación: ¿cómo y en qué medida la Consolidación podría representar algo de relevante para ese contexto de afirmación de un discurso liberal-republicano? La investigación que aquí se imparte trabaja con la hipótesis de que la obra tal vez no tenga servido a ese ideal, en un primer momento, tal vez porque representase una visión moderada en el pensamiento de los juristas respecto a los cambios legislativos de la época.

1.4 El método de trabajo - averiguación de los elementos de continuidad, en especial desde las ediciones de la Consolidação das leis civis

Desde el problema mencionado, de que dicho libro de Freitas, la Consolidación, hubiese recibido varias ediciones durante el período en que el gobierno republicano buscaba la afirmación de un proceso codificatorio, surgen algunas hipótesis de trabajo que pueden traer una luz a la comprensión de ese momento histórico. Notoriamente la República buscaba establecer la auto- 
nomía legislativa civil en el país con relación a las Ordenaciones Filipinas, vigentes en Brasil desde 1603 (en ese período, como parte del territorio portugués durante la unión dinástica con Castilla, 1580-1640), ordenaciones esas que representarían durante el siglo XIX en Brasil una continuidad con el pasado portugués. La Consolidación de Freitas, a juzgar por su forma y finalidad, no tenía la función de derogar o sustituir dichas Ordenaciones durante el Imperio: por el contrato de elaboración de tal recopilación quedaba claro que la función sería orientar la práxis forense presentando el último estado de la legislación civil. ${ }^{\mathbf{6}}$

Terminada la primera edición de tal obra en 1857 y aprobada por el gobierno en el año posterior, se publica con gran éxito editorial. ${ }^{7}$ Además, lleva a Freitas a un nuevo contrato, en enero de 1859, con el gobierno imperial, ahora buscando la elaboración del proyecto de Código civil, que se quedó conocido como Esbozo del Código civil. Desde ese contexto, la Consolidación se convierte en base para un proyecto de modernización legislativa impartido por el gobierno imperial; entre tanto, la función de ese texto en la práctica forense resultó ser otra - facilitaba la articulación del variado y complejo plano de leyes vigentes en el área civil en Brasil. Y eso explicaría su validez después de todos los cambios legislativos posibles dentro del espíritu de un discurso liberal-republicano muchas veces en contra de lo que estaba escrito en la Consolidación.

Para tales efectos, el método más adecuado en ese análisis será la averiguación de las repercusiones de la Consolidación en la práxis jurídica brasileña del inicio de la República, con vistas a sopesar principalmente elementos de la práctica jurídica que no se coadunaban con una visión meramente deductiva del ideario liberal republicano. Es lo que se buscará demostrar investigando el contexto de la publicación de esas nuevas ediciones de la obra,

6 En el texto integral del contrato, que Meira transcribe, están los puntos acordados entre el ministro contratante, Nabuco de Araujo, y el propio Freitas, como el objetivo de organizar y clasificar toda la legislación civil vigente en Brasil, comprendiendo las portuguesas aun vigentes y las surgidas desde la independencia en 1822, recopilando esas leyes en un demostrativo del último estado de la legislación de la época - Meira (1983) 94.

7 Hubo repercusión posterior en Argentina, a juzgar por publicaciones hechas traduciendo algunos textos de Freitas - Teixeira de Freitas (1900; 1909). De la misma forma, Martínez Paz hace la traducción de la Introducción de la Consolidación y publica en libro de su autoría Martínez PAZ (1927). 
y también desde los libros que se vinculan al tema, tanto de Freitas como de otros autores, y en revistas jurídicas de la época. ${ }^{8}$

\section{Intersecciones entre la práctica jurídica de la época y los trámites del proyecto de Código civil}

Con vistas a una comprensión más detallada del contexto de la publicación de la renombrada obra práctico-jurídica de Freitas, se pasará al análisis de los detalles de las ediciones de la Consolidación que se impartieron durante la República, como también de las repercusiones del debate parlamentar sobre el proyecto en las casas legislativas, y de las impresiones de autores que publican durante esos decenios republicanos.

\subsection{Las ediciones republicanas de la Consolidación de Freitas}

Con referencia a esa obra de Freitas, preliminarmente importa recordar de nuevo que tuvo tres ediciones durante la vida de su autor - la primera en 1857, todavía sin la venia de la comisión organizada para evaluar la obra, lo que sí se daría solamente en el año posterior; la segunda en 1865, justamente en el mismo año en que el propio autor fue duramente criticado cuanto a su más notable trabajo, el Esbozo del Código civil, por parte de la comisión que fue compuesta para ello; y la tercera en 1876.

Lo que sí se habla respecto de esas ediciones que tuvieron gran aceptabilidad en la práctica forense, muchas veces como guía práctico de organización de las fuentes del derecho civil, lo que era la intención del propio autor.

8 Así, por ejemplo, las revistas O Direito e Gazeta Jurídica, que se publican a partir de la década de 1870, tienen artículos de Freitas y recensiones respecto de sus libros. También estrictamente vinculado al éxito de la Consolidación en la práctica forense de la época estaría otro libro de Freitas que serviría como una revista de comentarios a esa obra; así, en 1877 se publica el libro Aditamentos a la Consolidación de las leyes civiles, en que el autor adopta el método de separar a los comentarios de acuerdo con las fuentes jurídicas básicas, definiendo las contribuciones que habían sido dadas por la legislación, la doctrina y la jurisprudencia Teixeira de Freitas (1877). 


\subsubsection{La reimpresión de la $3^{a}$ edición de la Consolidación en 1896}

Según ya se ha comentado, la última edición de la Consolidación que Freitas ha publicado en vida salió en el año de 1876, siete años antes de su muerte. En esa edición, el autor hizo cambios que entendía necesarios ante circunstancias nuevas, tanto con el tema de la manutención de la obra en el mercado editorial, como con vistas a la utilidad de la misma de servir a la práctica jurídica del período. Y además de eso, percibió que debería atender a factores nuevos, desde responder a críticas recibidas en las ediciones anteriores, como también nuevas ideas del propio autor sobre esa obra y la acomodación a los constantes cambios legislativos en la materia civil.

Ahora, respecto de la reimpresión que se hizo en 1896 por parte de la editorial que publicó los libros de Freitas durante su vida, la famosa Livraria Garnier, se puede afirmar que presenta algunas características peculiares. En términos generales, la citada publicación es realmente una reimpresión de la edición original, sin variación aparente cuanto a la publicación de 20 años antes. Entre tanto, dos puntos pueden ser realzados: (a) no presenta la totalidad de índices que se presentó en la edición original de 1876, sino solamente el primer índice, el alfabético; (b) lo que es indudablemente paradojal, no hace mención a la vasta legislación republicana que se impartió en aquellos años, sobre todo en los cinco años posteriores a la proclamación de la República en 1889. ${ }^{9}$

Con eso, más parece que esa reimpresión se basó en la receptividad que el texto ya tenía en la práctica forense de entonces; además, también se presume que la edición, del modo que se publicó veinte años antes, ya cumpliría con las necesidades del fuero, de donde la reimpresión bastaría para dar continuidad a su uso práctico-jurídico. Aquí se encuentra una señal de la disparidad entre el discurso y la práctica legislativa liberal de la República y la práctica jurídica que recepcionaba de una forma tradicional elementos de una práctica anterior en el Imperio.

9 Por todo, ver el texto de la reimpresión - Teixeira de Freitas (1896a); la comparación con la tercera edición de 1876 aclara las diferencias y demuestra lo que es simplemente copiado Teixeira de Freitas (1876). 


\subsubsection{La cuarta edición de la Consolidación en el año de 1910}

Cuando la República brasileña ya había ingresado en el inicio del tercer decenio de historia, pasados ya veinte años de la proclamación, y después de diez años en que se iniciaran los actos referentes a los trámites del proyecto de Clovis Bevilaqua (porque se ha presentado en 1899) que después se convirtiría en el Código de 1916, sale publicada una nueva edición de la Consolidación. Con relación a dicha edición, según se presenta en el propio texto, se trata ahora de una edición refundida (como se lee en el título de la obra), habiendo además la presentación, al final de todo el texto (y por eso se publicó en dos volúmenes), de la legislación republicana que se había publicado en materia civil hasta el mismo año de $1910 .^{\mathbf{1 0}}$

Es relevante recordar que esa legislación republicana, puesta como un apéndice, forma un conjunto en que se presentan las leyes civiles publicadas en esos años iniciales de la República sobre algunas materias vistas como claves para el debate republicano y que ya tenían repercusión en los últimos decenios del Imperio, como el casamiento civil (a causa de la presencia de inmigrantes de otros credos en Brasil, desde el Imperio, con que se solicitaba una respuesta ante la cuestión del predominio del casamiento católico en la legislación imperial) y la hipoteca (relevante para la temática del tráfico jurídico y económico necesario para implementar el progresso, en el sentido de la época y según los patrones europeos en boga). ${ }^{\mathbf{1 1}}$

De eso, se puede concluir que: (a) la edición de 1910 es, en el plano sistemático del autor, igual a la última antes de su muerte; (b) no hay ninguna referencia a quien organizó el apéndice de legislación republicana - se presume trabajo de la editorial, por el sentido de oportunidad de la publicación de la obra, pero sin que se hiciese algún tipo de cruce con el sistema de Freitas; (c) esa cuarta edición tiene algunas particularidades con relación a la edición anterior. $^{12}$

10 Vale recordar que es noticia en la Editorial Garnier de la venda de obras de Freitas que salieron a partir de 1878, y además algunas como reimpresión, e. g. la Doctrina de las acciones en 1902. Eso se percibe en el comienzo de la obra, antes de la portada, en página de la Editorial - Teixeira de Freitas (1910).

11 Así, el citado apéndice, empezando en la página 779 en el segundo volumen, va hasta la página 1109 , de donde resulta un total de 330 páginas con las leyes civiles de la República, como se nota el propio texto - Teixeira de Freitas (1910).

12 Algunos datos referentes a la estructura del libro: (a) se añade un índice del segundo volumen (que trata de los derechos reales) en la p.1111; (b) hay un índice muy incompleto del 


\subsubsection{La quinta edición que se publica en el año de 1915}

Respecto de esa edición, ya en el título se presenta como muy aumentada, en el sentido de que presenta la legislación republicana que se promulgó hasta el año de 1913; de hecho, del mismo modo que había ocurrido con la edición anterior de 1910, el libro viene publicado en dos volúmenes, en que la citada legislación republicana es puesta al final del segundo volumen. Además, la publicación ahora sale en otra casa editorial, la Jacintho Ribeiro dos Santos, que en la época tiene prestigio con sus publicaciones. ${ }^{\mathbf{1 3}}$

Hay una característica muy peculiar en esa edición de 1915, que es fundamental para comprender su papel en la práctica forense de entonces, que es la presencia de las anotaciones hechas por el organizador de la publicación, el civilista Martinho Garcez, que era un autor de renombre en aquel momento, en especial a causa de su importante libro sobre nulidades en derecho civil que se publica en 1914 .

Con referencia a dichas anotaciones de Garcez, que se presentan a pie de página y después de las notas del proprio Freitas al texto legislativo, al total llegan a contarse como más de una centena, siempre en la parte de la obra en que empiezan los artículos de la Consolidación. De su contenido, se percibe en tales anotaciones que hacen lo que se espera de una obra de otra época: hay una constante referencia a la legislación de la República, con que Garcez explica los cambios que se dieron en las reglas jurídicas y en los institutos de derecho civil en la época.

Como aspectos relevantes de la historia de esa obra, vale recordar que en la parte final en que se hacen propagandas de esa casa editorial, no hay ninguna referencia a la posibilidad de venta de otros libros de Freitas; y eso seguramente porque la mayor parte de sus libros, los que todavía estarían disponibles a esa altura para la venta, se deberían encontrar en las casas con que se trabajaban los demás libros del autor o en las posibles sucedáneas de las

apéndice (donde justamente están las leyes republicanas) en la p. 1113 (repitiéndose el índice en la p. 115 de una nueva numeración que empieza con el índice alfabético); (c) el índice alfabético (pp. 01-114 de la nueva numeración), es igual a la edición anterior y extrañamente no hace referencias a la legislación republicana - Teixeira de Freitas (1910).

13 A pesar de tratarse de una editorial distinta, sigue el patrón de las ediciones anteriores llegando a poner en el frontspicio de la portada la expresión Legislação do Brasil, con la que Freitas buscaba, en sus tres primeras ediciones, inculcar en el público la idea de que tenía legitimación para ello, dada por el gobierno imperial - Teixeira de Freitas (1915). 
mismas. Otros datos serían que hay una publicación al inicio de una documentación sobre la Consolidación, como el Decreto de autorización de 1858, con el informe de la comisión de la época que aprovaba el texto, y la autorización dada por el gobierno para la publicación de la tercera edición de la obra.

Como conclusión, se puede aseverar que: (a) el índice alfabético sigue siendo de la tercera edición; (b) el cruce con la legislación republicana se da simplemente en las anotaciones de Garcez. De esas sencillas aportaciones se deriva una noción compleja y disforme de la estructura de esa edición de la Consolidación. Eso porque el hecho de la publicación del mismo índice alfabético de la época final de Freitas puede interpretarse tanto en el sentido de falta de acuidad en el preparo de la edición como una reverencia a la obra en su status original. Pero, ante las leyes republicanas sinfín que se añaden a ese texto, sería de presumirse un cambio profundo en esos elementos de aproximación a la obra, como los índices. Mas eso sólo se daría por intermedio del cruce con la legislación republicana, que podría ocurrir en índices, pero que en esa edición solamente se daría en las anotaciones del organizador, Garcez. $^{14}$

\subsection{Las tramitaciones del proyecto Bevilaqua}

Para efectos de comparación, después de la presentación de las publicaciones de la Consolidación en el período, es importante recordar los pasos más significativos de la tramitación del proyecto de Código civil hasta su promulgación en 1916. De ese modo, se busca captar elementos de repercusión del discurso liberal-republicano, pues las casas legislativas brasileñas en ese período se presentaron como el espacio para el debate y la implementación de un factor típicamente de tal discurso, el proyecto de Código civil. Se debe recordar, con todo, que existe una historia anterior de los proyectos presentados en Brasil y que han malogrado, desde el Esbozo del Código civil de Freitas en la década de 1860 hasta un poco antes de ese proyecto que al final se convertiría en el Código, lo que a su vez no será objeto de examen en esa investigación.

14 Característica primordial de la edición es el aporte de Garcez en notas computadas en números romanos y con notorias diferencias (distinto estilo y mención a leyes republicanas) - Teixeira de Freitas (1915).

$190 \quad$ Alfredo de J. Flores 
Respecto del proyecto de Clovis Bevilaqua, primeramente se debe decir que fue elaborado durante el año de 1899, una vez que el autor del proyecto recibe el encargo en enero y termina llevando su versión final del texto al gobierno en noviembre del mismo año. Luego de su recebimiento, se constituye una comisión que hace muchos cambios y que entrega su versión final al gobierno, el que en noviembre de 1900 envía a la Cámara de diputados. Tal versión, el Proyecto revisto, tiene profundas diferencias con la versión inicial del texto en algunos puntos materiales; eso, entre tanto, no resultó en negación por parte de Bevilaqua a esa revisión, sino al contrario, él prontamente entendió que su proyecto debería tener una vida propia. ${ }^{15}$

En la Cámara de diputados, recibe informes de Facultades y críticas en revistas hasta la reordinación del texto y enmiendas de parlamentares con la Comisión de los 21 (compuesta así a causa de cada Estado de la federación brasileña en aquella época), que dividió el trabajo en informes parciales, hasta la refundación en enero de 1902, con el Relatorio de Sylvio Roméro, el que es discutido en la Cámara en marzo de 1902. Pasado eso, sigue a la otra casa legislativa brasileña, el Senado, en donde, en abril de 1902, el renombrado senador Ruy Barbosa hace el informe cambiando el lenguaje del proyecto - lo que inicia una contienda filológica sobre el proyecto con su maestro, Ernesto Ribeiro, que sería tal vez el debate público de mayor repercusión respecto a ese proyecto, ya que, con las posiciones presentadas, otros personajes importantes publican sus opiniones en diarios de la época, poniendo el proyecto literalmente en el debate público. ${ }^{\mathbf{1 6}}$

Después de esa fuerte repercusión, con sus cambios en el proyecto en virtud de esos debates, ocurre un lapso temporal hasta que se constituye una nueva comisión del Senado para apreciación del texto modificado; su primera composición se da en mayo de 1908, pero cambia de nuevo en mayo de 1909 hasta que, en noviembre de 1911, va a surgir la cuarta comisión del Senado que termina el examen. En la secuencia, vuelve ese proyecto a la Cámara,

15 Recuerda Vampré que Bevilaqua se presentaba en las comisiones para dar su opinión y participar de los debates - VAMPRÉ (1917) XVIII. De los informes presentados al proyecto hace mención VAmpré (1917) XIX, donde reconocidos civilistas, tribunales, facultades e institutos jurídicos opinan sobre el texto.

16 Así, Bevilaqua en el Prólogo de su obra En defensa del proyecto de Código civil, ha demostrado varias repercusiones que su proyecto a tenido en la élite nacional y además en otros países, comprobando que el texto tenía fuerza de expresión en la sociedad - Bevilaqua (1906) VII-XII. 
donde, en diciembre de 1912, una nueva comisión se constituye y va presentar un informe en abril de 1913; en julio de 1915, se vota el proyeto y se envía al Senado por las enmiendas. En el Senado, esas enmiendas son discutidas y se envía a la Cámara más una vez para que, en agosto de 1915, se haga nueva comisión y votación de enmiendas. En una reunión conjunta de las dos casas legislativas, al final del mismo año se hace la redación definitiva y en $1^{\text {er }}$ enero de 1916 sale promulgado el código. ${ }^{17}$

A juzgar por la repercusión directa de las casas legislativas en el vida cotidiana del pueblo en el inicio de la República, se puede decir que la tramitación de tal proyecto tuvo sus momentos de penetración en las calles. Se habla eso llevando en cuenta por lo menos la implementación de una nueva legislación en los primeros años de ese régimen, lo que seguramente era de interés de la población en la época, y la presencia notable en los periódicos de las más altas figuras políticas, recordando que había una concentración política en la capital republicana de entonces, la ciudad de Río de Janeiro, y muchos de esos políticos influyentes eran propietarios de los periódicos de la época o tenían fuertes vínculos con esos medios.

\subsection{Apreciación de los juristas de la época}

De un modo general, los autores de esa época atestiguan notoriamente un debate público con movilización política de varios grupos en torno al Código, como siendo un consenso republicano. Además, hubo una activa participación del autor del proyecto, Clovis Bevilaqua, en la infinidad de reuniones y discusiones públicas impartidas en las casas legislativas con referencia a ese proyecto durante ese decenio y medio.

Así, por ejemplo, el reconocido autor brasileño, Francisco C. Pontes de Miranda, considerado por muchos como el más importante del siglo XX en Brasil, ha publicado en el año de 1928 un libro sobre la historia del derecho civil en Brasil, de título Fuentes y evolución del Derecho civil brasileño. ${ }^{\mathbf{1 8}}$ En la citada obra, Pontes de Miranda hace una evaluación del contexto del Código

La tramitación es comentada brevemente por VAMPRÉ (1917) XXIII-XXIX, donde se realza el informe de Ruy Barbosa presentado en 03 de abril de 1902, justamente tratando del lenguaje del proyecto y con la propuesta de cambio de palabras y de significados. Miranda (1981). 
civil de 1916 y pone en relación con las fuentes legislativas que serviran de base apuntando que los textos de Freitas fueron lo mejor que se hizo en América en el siglo XIX (recordando que habla de la Consolidación y del Esbozo), como también ha afirmado que el intento de Carlos de Carvalho de hacer una versión del trabajo compilativo de Freitas, más adecuada a la República y a las nuevas tendencias doctrinales, que tuvo el título de Nueva Consolidación de las leyes civiles, no logró éxito. ${ }^{19}$

Sobre el proyecto de Bevilaqua, apuntó que lo que «toca la realidad, viene de Freitas o de Coelho Rodrigues», recordando igualmente el proyecto que también resultó ser rechazado como el de Freitas, pero ya en la República, en 1896. Hablando Pontes de Miranda respecto a Freitas, menciona que la Consolidación era «amplia, erudita, fiel, en que se casan el espíritu de organización y la técnica codificadora, de modo a constituir admirable construcción». ${ }^{20}$ A su vez, Pontes de Miranda encuadraba el Código de 1916 que advenía del proyecto de Bevilaqua en la tradición liberal individualista. ${ }^{21}$

A su vez, el propio Bevilaqua, en sus textos de la época hizo sus elucubraciones sobre su proyecto, la tramitación en las casas legislativas, la inserción social del Código y las fuentes que había utilizado para la elaboración del proyecto. ${ }^{22}$ De ese modo, anota el autor que el Código civil se ha convertido en texto legitimado por la actuación de los parlamentares y demás protagonistas en los debates, en donde se manifestaría el carácter particular de la construcción histórica brasileña. ${ }^{23}$ Reconoce aun raíces de sus ideas en las obras de Freitas; así, respecto de la Consolidación, apunta que «hace

19 «Teixeira de Freitas foi, no século XIX, o gênio do direito civil na América. A Consolidação das leis civis, cujas notas são magníficas fontes de doutrina, e o Esbozo do Código civil representam o que de melhor se tinha, em direito civil, em toda a América» - Pontes de Miranda (1981) 63.

20 Pontes de Miranda (1981) 80.

21 Decía Pontes del Código que era «individualista, tímido e menos político, mais sentimental do que os outros, porém mais «sociável〉 e menos «social` do que devia ser, serve para que se lhe descubra a intimidade daquele pensar por si, que Teixeira de Freitas ensinou à Sul-América, e os traços de generosidade orgânica, de aferro leigo às instituições religioso-morais, de povo mais caracteristicamente jurídico do que todos os outros da América» - Pontes de Miranda (1981) 09.

22 En su obra Linhas e perfis jurídicos, el autor recopila textos referentes al proyecto y algunos artículos posteriores a la promulgación del Código - Bevilaqua (1930).

23 Ya en el texto El derecho en Brasil, de 1914, que hace parte de esa obra como un capítulo, anota que el Derecho brasileño tiene «su carácter particular» - Bevilaqua (1930) 43. 
síntesis viva de legislación secular», mientras que el Esbozo es un «monumento imperecible». ${ }^{24}$ Pero añade que los textos prácticos, útiles para la comprensión de las leyes, se vinculan a la experiencia constituida en el país. ${ }^{25}$

\subsection{La relevancia de la Consolidación, en términos generales}

Cuanto a la Consolidación de Freitas, en sus varias ediciones, es posible decir que alcanzó validez al menos desde tres puntos de vista: la forma de Código, la validez empírica y el sistema, con que se nota que son puntos basilares para la comprensión de la relevancia del texto compilativo. ${ }^{26}$ Primeramente, hay una validez empírica de la obra que es innegable, a juzgar por los testigos cualificados que se posee. Como ejemplo, las palabras del diplomata Joaquim Nabuco, hijo de uno de los políticos más influeyentes del Imperio y amigo personal de Freitas, José Thomaz Nabuco de Araujo, confirmando la penetración del texto de la Consolidación en la práctica jurídica de entonces. Además de eso, también relevante son las autorizaciones gubernamentales durante el Imperio, en que todas las ediciones de ese texto compilativo aparecía la venia gubernamental para la publicación. Y, por fin, las citas en la práctica forense, muy comunes y reconocidas por los autores posteriores a Freitas.

Cuanto al sistema, el tema central es el orden de clasificación de la materia, que es el punto central de todo el debate de Freitas con los demás autores del período. No se trata igualmente de un debate sobre el Código: según se percibe de la obra de Freitas, no se resume su pensamiento a la elaboración del proyecto de Código civil, hasta porque él mismo habría de cambiar de idea al final. ${ }^{27}$

24 Bevilaqua (1930) 59.

25 Dice el autor: «E qual será a physionomia do direito brasileiro? Dizer que, depois de termos organizado o paiz inspirando-nos, para tracejar a nossa Constituição monarchica, em idéas francezas e inglezas, fundamos a republica, segundo os moldes da federação presidencialista norte-americana, seria ficar áquem do nosso objectivo, porque essa descorada informação me deixaria na superfície dos factos, que pretendo observar. Penetrando, porém, mais intimamente, no cerne de nossa organização politica, a justiça me levará a reconhecer que, se os moldes constitucionaes, nesses dois momentos decisivos da vida nacional brasileira, não foram productos do solo, creações originaes da raça, receberam modificações valiosas, que tornaram possivel a sua adaptação ao meio patrio, e revelarão, a todo o tempo, a impressão do nosso modo de comprehender e sentir os phenomenos sociaes»-Bevilaqua (1930) 49.

26 Siguiendo aquí a Samuel Barbosa en la caracterización - Barbosa (2008) 370.

27 Cuanto a la cuestión del sistema, vale recordar que el propio autor ha rechazado en 1867 el modelo que ha puesto en el Esbozo y empezó a hablar de la distinción entre un Código geral 


\section{Conclusión}

La cuestión central de esa investigación sería comprender lo que está por detrás de la publicación de textos de compilación legislativa (en particular las ediciones de la Consolidación, pero hay que recordar la Nueva Consolidación de Carlos de Carvalho) en el período de debate del proyecto de Código civil. Partiendo de esa constatación, se concluye que: (a) el discurso de modernización jurídico-política en Brasil realmente fue ejecutado en la República mediante las modificaciones legislativas impartidas luego al inicio del nuevo régimen; (b) desde la perspectiva de una historia de los libros, se puede percibir que las ediciones de la Consolidación durante la República son hechas sin que tenga mayores retoques; ${ }^{\mathbf{2 8}}$ (c) normalmente, la legislación republicana se añadía al final (como se ve en la $4^{\mathrm{a}}$ y en la $5^{\mathrm{a}}$ edición); y (d) esas ediciones no atienden a la idea que tenía Freitas de publicar el texto de la Consolidación con la presencia de los índices de las ediciones en vida de Freitas (así, la $3^{\mathrm{a}}$ reprint, la $4^{\mathrm{a}}$ y la $5^{\mathrm{a}}$ ); (e) comentarios a pie de página, sólo con Garcez en la $5^{\text {a }}$ edición.

Hay que recordar que hubo además las dos ediciones de la Nueva Consolidación de Carlos de Carvalho (en 1899 y en 1915), y además ese autor hace el cruce entre los textos de Freitas, pero respetando la estructura del debate alemán y el sistema del BGB, trabajando toda la legislación republicana incipiente. ${ }^{29}$

Sobre la función de la Consolidación durante el proceso codificatorio imperial, es posible afirmar que hay sí diferencia con el contexto republicano, aunque en los dos casos el texto tenga convivido con proyectos de Código en debate. El caso del proyecto de Bevilaqua es algo distinto, porque se trata de la

y el Código civil (ahora como Código de todo el derecho privado) - Teixeira de Freitas (1896b).

28 La perspectiva que se buscó utilizar sobre la valoración de los detalles del libro tiene inspiración en lo que Darnton intenta describir como «la historia del libro» - Darnton (2010) 189-219.

29 Respecto al texto de Carvalho, la edición de 1915, con relación a la primera, se hace sin prácticamente cambios (hay una editoración distinta, en que aparecen más páginas) Carvalho (1915). A pesar de todo ese esfuerzo del autor para atender a las necesidades de la época, Pontes habla que no fue exitosa su repercusión en el fuero, según ya se habló: "Carlos de Carvalho, já na República, escreveu a Nova Consolidação das leis civis, em que procurou atualizar, resumir e afeiçoar a novas tendências doutrinárias a Consolidação de Teixeira de Freitas. Livro paciente, mas falho» - Pontes de Miranda (1981) 63. 
implementación de una estructura de derecho civil que era necesaria para la modificación hacia la modernización.

Eso se explica por el modelo constitucional de los Estados Unidos que sirvió de base a la élite brasileña de entonces; su repercusión en la legislación infraconstitucional fue notoria, al menos formalmente (como al inicio de la República, con la posibilidad de que cada Estado federado presentase proyectos de Código de proceso), lo que luego se ha abandonado a favor de la afirmación de una modernidad múltiple, aplicable de modo especial a Latinoamérica. $^{30}$

Entre tanto, hay otros elementos que explican ese éxito de la Consolidación: así, el papel de la estabilidad institucional que se presenta en el Segundo Imperio brasileño y la consolidación de nichos de debate y decisión. Una vez que hay la supervivencia de las instituciones públicas en el Imperio, los elementos se encajan institucionalmente en las clases de trabajos intelectuales, es decir, (a) se consolida la hermenéutica, mediante el Consejo de Estado y los Tribunales de Relación; (b) en la política, hay el predominio de gabinetes ministeriales y partidos políticos; (c) se fortalece la academia y la vida universitaria (con sus Facultades).

Claro está que existen además los factores de la falta de estabilidad política, los cuales generan tópicos de debate público desde la Guerra del Paraguay (1864-1870) y el movimiento republicano (post-1870). Añadiéndose a eso, los problemas internos, como el reconocimento de derechos civiles a inmigrantes (la cuestión de la tutela en los actos civiles más importantes casamiento, esclavitud) en el período anterior a la República. Por fin, la política de federación y provincias (con el eterno tema de la centralización y descentralización en Brasil). ${ }^{31}$ Pero eso no entraña en la práctica forense; en

30 Respecto a eso, ha apuntado Alessandro Somma que «Per un altro verso si possono invece valorizzare i tentativi di discutere di modernizzazioni al plurale, si può cioè considerare l'intreccio tra diritto indigeno e sviluppo della democrazia e del capitalismo come il tratto distintivo della modernizzazione latinoamericana rispetto alle altre forme di modernizzazione, quella occidentale in testa. È quest'ultimo il punto di vista che utilizzeremo» - SоммA (2012) 18.

31 La propuesta de aplicación de una metodología histórico-jurídica en perspectiva global sería válida para explicar los procesos brasileños de reconstrucción de modelos legislativos importados de Europa ante la práctica tradicional forense en el Brasil republicano; de hecho, a varios de esos puntos se buscó tratar en la presente investigación. Y como habla Thomas Duve, importa observar a los espacios y los instrumentos - Duve (2012) 54. 
definitiva, el paso relevante para sanar el asunto sería identificar el sistema lingüístico según las categorías y los protagonistas en cuestión.

\section{Bibliografía}

Barbosa, Samuel (2008), Complexidade e meios textuais de difusão e seleção do Direito civil brasileiro pré-codificação, in: Fonseca, Ricardo, Airton Seelaender (orgs.), História do Direito em perspectiva, do Antigo Regime à Modernidade, Curitiba: Juruá, 361-373

Bevilaqua, Clovis (1906), Em defeza do projecto de Codigo civil brazileiro, Rio de Janeiro: Livraria Francisco Alves

Bevilaqua, Clovis (1930), Linhas e perfis jurídicos, Rio de Janeiro: Livraria Freitas Bastos

Carvalho, Carlos de (1915), Direito civil brazileiro recopilado ou nova Consolidação das leis civis vigentes em 11 de Agosto de 1899, Porto: E. Nogueira

Darnton, Robert (2010), A questão dos livros, passado, presente e futuro, tradução de Daniel Pellizzari, São Paulo: Companhia das Letras

Duve, Тномаs (2012), Von der Europäischen Rechtsgeschichte zu einer Rechtsgeschichte Europas in globalhistorischer Perspektive, Max Planck Institute for European Legal History n. 2012-01. Available at SSRN: http://ssrn.com/abstract $=2139312$

Lynch, Christian (2010), A República Aristocrática o discurso político conservador da Primeira República, in: Anais do VII Encontro da Associação Brasileira de Ciência Política (ABCP) - trabalhos completos, 01-45, http://cienciapolitica. servicos.ws/abcp2008/arquivos/22_7_2008_17_28_27.pdf

Lynch, Christian (2011), O momento oligárquico: a construção institucional da República brasileira (1870-1891), in: História Constitucional 12, 297-325, http://www.historiaconstitucional.com

Losano, Mario G. (1974), La scuola di Recife e l'influenza tedesca sul Diritto brasiliano, in: Tarello, Giovanni, Materiali per una storia della cultura giuridica, vol. IV, Bologna: Società Il Mulino

Martínez Paz, Enrique (1927), Freitas y su influencia sobre el Código civil argentino, Córdoba: Imprenta de la Universidad

Meira, Sítvio (1983), Teixeira de Freitas, o jurisconsulto do Império, 2 ed., Brasília: Cegraf

Pontes de Miranda, Francisco Cavalcanti (1981), Fontes e evolução do Direito civil brasileiro, 2 ed., Rio de Janeiro: Forense

Saldanha, Nelson (1985), História e sistema em Teixeira de Freitas, in: Revista de Informação Legislativa 85, 237-256

Somma, Alessandro (2012), Le parole della modernizzazione latinoamericana: centro, periferia, individuo e ordine, in: Max Planck Institute for European

El proyecto de modernización del ideario liberal republicano en Brasil en cuestión 
Legal History n. 2012-05. Available at SSRN: http://ssrn.com/abstract=2169420 or http://dx.doi.org/10.2139/ssrn.2169420

Teixeira de Freitas, Augusto (1876), Consolidação das leis civis, $3^{\mathrm{a}}$ ed., Rio de Janeiro: B. L. Garnier

Teixeira de Freitas, Augusto (1877), Additamentos à Consolidação das leis civis, Rio de Janeiro: Instituto Typographico do Direito

Teixeira de Freitas, Augusto (1896a), Consolidação das leis civis, $3^{\text {a }}$ ed. reimpr., Rio de Janeiro: H. Garnier

Teixeira de Freitas, Augusto (1896b), Codigo civil - proposta do Dr. Teixeira de Freitas sobre um novo plano para o mesmo Codigo, in: O Direito 71, 321-328

Teixeira de Freitas, Augusto (1900), Código civil - Proyecto, traducción de Arturo Pons, Buenos Aires: Imprenta El Hogar y la Escuela

Teixeira de Freitas, Augusto (1909), Código civil, obra fundamental del Código civil argentino, 2 tomos, Buenos Aires: A. García Santos

Teixeira de Freitas, Augusto (1910), Consolidação das leis civis, 4 a ed. 2 vol., Rio de Janeiro: Garnier

Teixeira de Freitas, Augusto (1915), Consolidação das leis civis, $5^{\text {a }}$ ed. 2 vol., Rio de Janeiro: Jacintho Ribeiro dos Santos

VAmpré, Spencer (1917), Codigo civil brasileiro (annotado á luz dos documentos parlamentares e da doutrina), São Paulo: Livraria e Officinas Magalhães 


\section{Republic and Strike Action in the Beginning of the $20^{\text {th }}$ Century: A Debate between the 1906 Strike and Legal History}

\section{Introduction}

From the railroad workers strike in 1906, the present article aims to prove that the right to strike was an enshrined right in the doctrine, in the working class and in the Brazilian jurisprudence in the beginning of the $20^{\text {th }}$ century, in spite of the violent response by the Executive Branch. To go on strike was a right that was met with the strength of the police and the army.

Thus, this article intends to demonstrate how the Legal History is not a history towards the progress, a walk of the reason or an evolutional history: "law must be understood in its time, and not simply as a walk towards progress, it is not possible to say that the past was better or worse, it simply changed, it was simply different and the task of the historian is to constantly make history complex." ${ }^{1}$ The task of the legal historian is to notice the contradictions, ambiguities and tensions that coexist within the legal norm. Therefore he may understand the past with its characteristics and singularities, and not as a preview of the present: the past ceases "to be a precursor to the present, a rehearsal for solutions that have a complete development of the present. And, with this, it ceases to have to be read in the perspective of what came next. The past is liberated from the present. Its logic and categories gain depth and autonomy."

That is, the past ceases to be seen under the logic of the present and is understood in its peculiarities, in its contexts, bringing the perception that "legal history is one amongst the thousands that can be possible, legal history is the law and what was made of it." 3

1 Silveira Siqueira (2011) 21.

2 Hespanha (2005) 43.

3 Silveira Siqueira (2011) 24.

Republic and Strike Action in the Beginning of the $20^{\text {th }}$ Century 
This way, it is intended to deny thoughts that "the right of law never existed" or that going on "strike has always been a crime". The intention is to show how this right existed with the violence and prejudice that were inflicted upon it, using legal history, not as a present "that never came", but as learning from experience for the future. Here history is used for the future, so that, with past experiences, it may be possible to discuss possible (not believing that history repeats itself or is the same) future experiences.

\section{The strike of 1906}

The strike of 1906 involved two of the main railroad companies in the state of São Paulo: Paulista and Mogyana. Considered to be the largest strike in Brazil until then, the movement initiated in May and ended in June impeded the transportation of coffee - main export product in Brazil -, of people, of correspondence and banking services.

Literally, a large part of the economic activity was paralyzed with this strike. Initiated against the abuse of the chief engineers, the essence of the strike was the repudiation to the "violation of workers' dignity". In the Strike Manifest of May $15^{\text {th }}, 1906$, the Liga Operária (Workers' Guild) summoned the workers to fight against the "harassment", "pay cuts" and "dismissals" that "offend our dignity as honest workers, which do not consider ourselves slaves and neither want to submit ourselves to the arbitrariness of tyrant superiors, which cannot and should not continue," fighting "with the steadiness and enthusiasm that our cause render us." ${ }^{\mathbf{4}}$ Having the workers that "violated the workers' dignity" dismissed, the strike ends without the grant of the claims.

Thus, the strike of 1906 was chosen, because it is an excellent moment to understand the legal tensions that existed around the right of strike. The strike of 1906 involved the Workers' Guilds, lawyers, the state of São Paulo, the Police, the Army ... It is a rich movement that can illustrate a little of the legal experiences at that time.

4 Published in the Newspaper Commércio de São Paulo on 15th May, 1906, in the Newspaper Cidade de Campinas on 16th May, 1906 and in the Newspaper A Terra Livre on 16th May, 1906. 


\section{The legal treatment of the strike in the beginning of the 20th century}

Sixty days after the publication of the Penal Code of 1890 that criminalized the strike in articles 205 and 206, the interim government altered the text through the decree number 1162:

The Chief of the Interim Government of the Republic of the United States of Brazil, considering that the text of articles 205 and 206 in the Criminal Code may in its exercise give rise to doubts and wrongful interpretations to establish the indispensable comprehensibility, mainly in the penal laws, decrees:

Article 1 - The articles 205 and 206 and their paragraphs are thus written:

Article 205 - Divert workers from the places of work, by means of threats or embarrassment:

Penalty - imprisonment from one to three months and fine of $200 \$$ to $500 \$ 000$. Article 206 - Lead or provoke ceasing or suspension of work by means of threats or violence, in order to enforce on workers or bosses the raise or cut in labor or salary: Penalty - imprisonment from one to three months. ${ }^{5}$

According to the new text, the peaceful strike ceased to be a crime, remaining as a crime only the violent strike. Under penal code, call workers to strike, without threats and embarrassment, was licit. Evaristo de Moraes, in 1905 , commented on the penal code, "under penal law in exercise in Brazil, the right to strike is plainly recognized." (...) "just like a worker may individually stop working, many workers have the right to refuse the effort of their arms to the call to meet the bosses' needs. Nor would it be compatible with a republican government the denial to this right, which originates in the economic settings of our time."

5 Original: O Chefe do Governo Provisório da Republica dos Estados Unidos do Brazil, considerando que a redacção dos arts. 205 e 206 do Codigo Criminal pode na execução dar logar a duvidas e interpretações erroneas e para estabelecer a clareza indispensavel, sobretudo nas leis penaes, decreta:

Art. 1.e Os arts. 205 e 206 do Codigo Penal e seus paragraphos ficam assim redigidos:

Art. 205. Desviar operarios e trabalhadores dos estabelecimentos em que forem empregados, por meio de ameaças e constrangimento:

Penas - de prisão cellular por um a tres mezes e de multa de $200 \$$ a $500 \$ 000$.

Art. 206. Causar ou provocar cessação ou suspensão de trabalho por meio de ameaças ou violencias, para impôr aos operarios ou patrões augmento ou diminuição de serviço ou salario:

Penas - prizão cellular por um a trez mezes.

6 Moraes (1905) 57-58. 
If the 1891 Constitution assured the right to gather (Art. $72 \rrbracket 8$ th), the freedom of speech (Art. $72 \rrbracket 12$ th), and of profession (Art. $72 \rrbracket 24$ th), to go on strike, with the absence of a penalty, was a right. If the worker can work, so he can also choose not to work and gather to express their thoughts. Thus, to understand that the strike was a right, seemingly, is compatible to a State influenced by the liberalism of the beginning of the $20^{\text {th }}$ century.

On the 1891 Penal Code, Nelson Hungria commented:

The object of legal protection is, here, the freedom of work against the imposition of strike or lock-out. The crime is the embarrassment to the ceasing (complete paralyzing or for a long time) or the suspension (transitory paralyzing) of work. Strike and lock-out are not crimes in themselves: they represent, on the contrary, a right, and the opposition to exercise them should be considered illegal (art. 180 Consol.). What the law punishes is the forcing or coercing of workers to strike, or the bosses to lock-out, to coalition. ${ }^{7}$

In the same sense, the Federal Supreme Court stood, in 1920, in judging the Habeas Corpus of a foreign striker expelled from the country by the São Paulo government for taking part in the 1906 movement.

Considering that the peaceful strike is a right that can be freely exercised by the worker, and that the exercise of a right in any free and law-enforced country is not a crime, nor does it place the individual in a situation of being considered a pernicious element to society or a disturber of the public order.

By the analysis of the documents submitted it is proved, in evidence, that the individual, intervening in the Mogyana strike with the intention of calming down the exalted strikers, did not commit an act, against people or goods, defined by penal Law, and nor any other manifestation with words or fact, became a "pernicious element to society", in which he has lived for twenty-four years, and in which he supports 7 Brazilian children.

Considering that the individual is Brazilian, therefore, has Brazilian children and an estate in Campinas, $u t$ document page 27, and is a tax-payer for municipal property.

Considering that, in this situation, the Constitution of the Republic, in the article 96, paragraph 5, considers the foreigner a naturalized Brazilian under any legal effects, and the expelling order does not apply to Brazilians.

7 Hungria (1936) 385. Original: “O objecto da protação penal é, aqui, a liberdade de trabalho contra a imposição da gréve ou do lock-out. O crime é o constrangimento á cessação (paralyzação definitiva ou por longo tempo) ou á suspensão (paralyzação transitoria) do trabalho. A greve e o lock-out não são crimes em si mesmos: representam, ao contrario, um direito, devendo mesmo considerar-se constrangimento illegal (art. 180 Consol.) a opposição ao seu exercicio. O que a lei pune é o forçar ou coagir os operários á greve, ou os patrões ao lock-out, á coalizão.” 


\section{The Federal Supreme Court}

GRANTS the appeal, so that all and any embarrassment to the individual be ceased, from the expelling decree. Expenses "ex-causa".

Federal Supreme Court, June 14th, 1920. - Pedro Mibielli, Reporting Judge: even if the individual were a foreigner, proven that he is a resident, I would grand the "habeas-corpus" under the terms of the article 72 of the Constitution of the Republic. - Pedro Lessa. - Leoni Ramos. - Pedro dos Santos. - Viveiros de Castro Godofredo Cunha. - Sebastião de Lacerda. - Muniz Barreto. - Germenegildo de Barros - João Mendes. ${ }^{\mathbf{8}}$

The law, the Constitution, the doctrine and jurisprudence agreed that the peaceful strike was a right of the worker. It was interesting to notice that such understanding was also shared by the workers and the bosses. But it is also necessary to understand that it is not possible to claim that all doctrine or all jurisprudence was for the right to strike. Decisions such as the one of the Justice Court of São Paulo (and indoctrinators like Baptista Pereira), in several occasions, criticized the right to strike or illegally deterred its exercise. ${ }^{9}$

8 Published in the Revista do Supremo Tribunal Federal in October, 1920, Fasc. 1, volume XXV, Rio de Janeiro, 149-150 (HC number 5.910). Original: Considerando que a gréve pacifica é um direito que póde ser livremente exercido pelo operario, e que o exercicio de um direito em qualquer paiz livre e policiado não constitue delicto, nem colloca o seu titular em situação de ser considerando um elemento pernicioso á sociedade e compromettedor da tranquillidade publica;

Considerando que dos documentos offerecidos se prova, á evidencia, que o paciente, intervindo na gréve da Mogyana com intuito de acalmar os animos exaltados dos grévistas, nem um acto praticou, isoladamente contra pessoas e cousas, definido pela Lei penal, e nem qualquer outra manifestação por palavras, ou factos teve como indicativo de ser elle um "elemento pernicioso á sociedada", na qual vive há vinte e quatro annos, e em cujo meio presta assitencia a 7 filhos brasileiros,

Considerando que o paciente é brasileiro, porquanto, tem filhos brasileiros, e possue um immovel urbano em Campinas, ut documento de fls. 27, pelo que é contribuinte dos cofres municipaes por impostos devidos pela propriedade predial.

Considerando que, nessa situação, a Constituição da Republica, no art. 96 parágrafo 5, considera o extrangeiro naturalizado brasileiro para todos os effeitos legaes, e que a lei de expulsão invocada não se applica a brasileiros.

O Supremo Tribunal Federal

DÁ PROVIMENTO ao recurso interposto, para que césse todo e qualquer constrangimento contra o paciente, oriundo da portaria de expulsão. Custas "ex-causa."

Supremo Tribunal Federal, 14 de Junho de 1920. - Pedro Mibielli, Relator: ainda que extrangeiro fôsse o paciente, provado que é residente, eu concederia o "habeas-corpus", no termos do art. 72 da Constituição da Republica.

9 Some of these rulings are reproduced in Leme (1984). 
Yes, it was possible to notice Judiciary rulings, not expressly opposed to the understandings of the Federal Supreme Court, but that did not recognized the right to strike as a right that could be exercised.

From pamphlets and manifests used in the strike of 1906 it is possible to notice the contradictions and tensions of the time. It is also possible to notice the reactions of the strikers, the company-owners and the government of the State of São Paulo at the time.

The manifest of the Workers' Guild of Jundiaí, published on May $19^{\text {th }}$, 1906, on the first page of the Jornal Commércio de São Paulo, stated: "our cause is a just one and a saint one, and for this reason we ought to work together and in mutual agreement to win the right that assists us and safeguards our dignity as men." The workers believed they were exercising a right.

On May $19^{\text {th }}, 1906$, Joaquim da Silveira, Joaquim Barros and Crizanto Pinto published a Positivist Manifest in the city of São Paulo. ${ }^{\mathbf{1 0}}$ For the positivists: "strikes do not constitute a crime, they are not punishable acts; on the contrary: they constitute a normal resource that the working class may use against the abuse of the industrial bosses and they are originated in the principle of professional freedom, established in the Constitution." Not being a crime, the "role of the police is to maintain the order at all times and ensure the complete freedom for the ones who wish to go back to work as well as the ones who decide to maintain the strike." The intention of the positivists was to reach a consensus, and through their manifests it is clear the recognition of the right to strike.

On May $25^{\text {th }}$, 1906, the Newspaper O Estado de São Paulo publishes a letter from the lawyer from Cia. Paulista, Pedrom Villaboim, who defended the actions against the strike:

The action of mere defense, agreed upon between Company and the government to safeguard the property already damaged by some of the so-called strikers, to ensure the safety of public transportation that relies on roads and to ensure the freedom to work to those who do not join the abstention, is being pointed out as a violence against the right to strike and some requests to courts to protect the workers against a fantasized oppression are being made. (...) Well, until now, no-one from the Company or from the government has refused the right to strike to the workers

10 Attached to the thesis by Leme (1984) 280-281. 
of Cia. Paulista; no-one has denied them the right to, via an agreement or a collective resolution, refuse their services to the company. ${ }^{11}$

The lawyer claimed that no-one in the Company had refused the right to strike and that the company itself was acting to fight, with the support of the government, the violent strike. He continued, however, to claim that the Company had respected the rights of the workers and that the workers had not respected the rights of the Company, by disenabling machines, pulling out rails, etc ... Therefore the strikers did not "limit themselves to the exercise of a right; they criminally attacked the Company, acts punishable by the Penal code." That's why the police acted "within the limits of extreme moderation", "without an act of violence to anyone." Concluded the lawyer: "what is in question, therefore, is not the right to strike. Against this licit and powerful weapon of vindication, no-one rebels, on the contrary, it is considered by all with great sympathy."

It was not prudent to deny the right to strike. That is why, in all attacks against strikes, there was an attempt of "legitimization", with claims that the strike was not peaceful. The right to strike was "recognizes", but fought under the allegation that the strike was a violent one.

By saying that they respected the right to strike and fought the violent strike, the Companies, alongside the state and federal governments, used the force against all strike movement. The strike, violent or not, was considered a disturbance to public order that should be fought.

\section{A violent response to the exercise of a right}

All generalization impoverishes any debate, and thus the strike of 1906 will be mentioned only to cite possibilities of action during a strike. It will be used to illustrate and to generalize all strikes at the time, which should be analyzed with their own peculiarities.

11 Original: A ação de mera defesa, combinada entre a Cia. e o governo para resguardar as propriedades já danificadas por alguns dos chamados grevistas, para garantir a segurança do transporte ao público que se utiliza das estradas e para assegurar a liberdade de trabalho aos que não acompanham a abstenção, está sendo apontada como uma violência ao direito de greve e já se anunciam pedidos de garantia aos tribunais contra a fantasiada opressão dos operários. (...) Ora, até aqui, ninguém da Cia. ou do governo recusou esse direito de greve aos trabalhadores da Cia. Paulista; ninguém lhes negou o direito de, por um acordo ou por uma resolução coletiva, recusarem seus serviços à empresa. 
The "first reaction of the Companhia Paulista (when made aware of the strike) was to intimidate the strikers, threatening to fire them, besides asking for the police support from the state government." ${ }^{\mathbf{1 2}}$ One day after the beginning of the strike, the president of Cia. Paulista headed out to Campinas taking with him " 50 police soldiers to guard rails and bridges, threatened by the exalted strikers." 13 "One of the most commonly used ways by the bosses to contain these strike manifestations was repression. At the slightest sight of a strike, they would alert the police, intent on maintaining order, and ensure the safety of the company goods and facilities." ${ }^{14}$

Violence would be used against the strikers, no matter if the strike was a peaceful one. On May $17^{\text {th }}, 1906$, the newspaper Cidade de Campinas, reports that the situation in Jundiaí and Rio Claro is of "perfect tranquility". In the city of Campinas, the newspaper informs, the only moment tempers flared was when soldiers that were arriving from São Paulo "exceeded themselves and hit - with the butts of their pistols - some people" who were shouting against the police officers who had arrived from the capital: "Mr Bandeira de Melo (deputy from Campinas) reported the fact to the commander so that he could solve the problem." In the beginning of the evening the strikes held a meeting, with around 2,000 people, in which they decided to maintain the strike. The police deputy was also present at the meeting: "Mr Bandeira de Mello also made a quick statement, advising the strikers to keep calm. The meeting was adjourned on the best fashion, at $7 \mathrm{pm}$.'

The debate about the strike was also happening at the courts. On May $23^{\text {rd }}, 1906$, the lawyer representing the Workers Guild from Jundiaí, Affonso Celso Garcia, presented the preventive habeas-corpus ${ }^{15}$ in favor or the members of the Guild, threatened with imprisonment. The lawyer stated that "one of the forces that moves the working class to claim their rights, undeniably, is the strike" and that "no government will prohibit a strike without damaging the freedom of labor, the freedom of association, the freedom of gathering, three rights that the supreme law of educated peoples enshrines as

12 Nomelini (2010) 164. On the same day, the Second Assistant Chief of Police of the state of São Paulo, Augusto Pereira Leite, assures that those who want to work will have the protection of the police, "as well as maintaining the order, in case of disturbance."

13 Zambello (2005) 84.

14 LeME (1984) 100.

15 The Workers Guild requested the publication of the habeas-corpus in the Newspaper Commércio de São Paulo, on May 24th, 1906, on pages 1 and 2. 
a precious conquest." Thus, "the strikes, which were punishable in the past, are today, when peaceful, an incontestable right in the civilized world." As the strikes were not prohibited in Brazil, the preventive habeas-corpus was filed so that the members of the Guild could not be arrested illegally by the police for the exercise of a right. ${ }^{\mathbf{1 6}}$

The claim of the lawyer made a statement for the strike as an exercise of claims from the workers and defended the right to strike, enshrined in the "civilized world". Other habeas-corpus were filed under the allegation that there was also "a rupture in the constitutional assurances to freedom of action, thought and movement, maintained by any republican regime." ${ }^{\mathbf{1 7}}$

"Strikes, at that time, were treated like rebellions, and as they were fought vigorously, the government and the capital showed their strength over labor." ${ }^{18}$ The right to gather, assured by the Constitution of the Republic, was also questioned: "with the intention of maintaining the order, people started to lose the possibility of freely associate to complain and demand measures from public institutions, when feeling wronged." ${ }^{19}$

On May 23rd, 1906, in the newspaper Il Secolo, the Union of Graphic Workers protested against the dissolution of a meeting by the police. For them, "the police attacks the constitution of the country, since we are not in state of siege, and since this police violence is another provocation against the workers, the Union protests (...) Free laws are created in this country, a peaceful regime, without a shadow of tyranny and oppression, and however, these laws are not enforced and armed forces, in a repulsive partiality, tries to suffocate the voices of workers in order to better serve the rich."

The right to strike, the laws or the constitution were of little concern. The strike was considered a disturbance of the peace and would be described as war, ${ }^{20}$ as a total violation to the normality. It did not matter if the service was public or private, fighting the strikes was also a task of the State:

16 Silveira Siqueira (2011) 105.

17 LeME (1984) 119.

18 LEME (1984) 192.

19 LeME (1984) 192.

20 The newspaper Minas Geraes, on May 21 ${ }^{\text {st }}$, 1906, describes the atmosphere of war in the state of São Paulo, reporting the movement in the barracks and the soldiers called to fight the movement. On May $23^{\text {rd }}, 1906$, the same newspaper reports the censorship that the telegraphs from São Paulo were under, as well as the request of help made by the Governor of São Paulo, Jorge Tibiriçá, to the president of the Republic, Rodrigues Alves. The newspaper also reports the visit of the Chief of Police to the house of the President of 
Given the circumstances, the president of the state telegraphed the president of the Republic, Rodrigues Alves, notifying the joining of Mogiana and probable joining of Docas de Santos and people from the Central. As a reply, the president of the Republic sent warships to the harbor of Santos, and the police were ordered to take even more drastic measures. ${ }^{21}$

On May $21^{\text {st }}, 1906$, the battle cruiser "Barroso", a very modern warship at the time, arrived at the harbor of Santos. ${ }^{22}$ The cruiser "Tiradentes" was sent to Santos on May 26th, $1906 .{ }^{23}$ Warships and soldiers were sent to stop the strike from spreading.

\section{Conclusions}

It is possible to notice that the positivation of a right does not ensure its exercise, that is, the positivation of a right is merely a part of the long process of struggle of the constitution of a right. This means that a right is the result of struggles for its recognition. Only through recognition may a right be exercised to and for all.

In this specific case, the right to strike, although enshrined on legal spheres, was constantly violated by a State that worried far more with the economic damages then the rights at that time. To meet its demands, the State violated citizens' rights.

In the same way, it is important to realize that the right to strike was on the consciousness of the workers and in part of the Brazilian society. Despite not being positivized, it was recognized as an existing right, which did not prevent it being fought by the illegality of the State.

By being in the consciousness of part of the population, it is possible to recognize the State as the main agent of the illegal actions and it brings to life a sense of legality towards the right to strike. The criminalization of

Companhia Paulista and elected-mayor of São Paulo, Antonio Prado. Prado claimed to be satisfied with the readiness of the police in ending the strike.

21 Original: Diante dos fatos, o presidente do Estado telegrafou ao presidente da República, Rodrigues Alves, notificando a adesão da Mogiana e a provável adesão das Docas de Santos e do pessoal da zona da Central. Em resposta, o presidente da República enviou 'vasos de guerra' para o porto de Santos e a polícia teve ordens para adotar medidas cade vez mais enérgicas. Leme (1984) 100-101.

22 Reported by Jornal Commério do Rio de Janeiro on May 22 ${ }^{\text {nd }}, 1906$.

23 Reported by Jornal Commério do Rio de Janeiro on May $27^{\text {th }}, 1906$. 
strikes in the 30s does not extinguish the legal sense and consciousness towards this strike. That is, the criminalization shows how the process of creation of a right is also vulnerable to setbacks, non-linear processes, mishaps and contradictions. ${ }^{24}$ Such as it is, legal history is not linear, nor is it a path to progress, and even less cunning of reason.

Legal history is made of flaws, contingencies, violence, and, essentially, the fight for rights. A right is constituted in a long struggle process and not by simply positivation. Thus it is possible to state that the right to strike existed in the First Republic and that its criminalization in the New State did not put an end to its exercise and the constant struggle for it.

These realizations have led us to, more and more, multiply the sources to create legal history. If laws, jurisprudence and doctrine were merely showing a "romantic" view of the time, that is, by these sources was law assured, it is imperative to verify which experiences dealt with each right. That is why it is interesting to see how, for instance, social movements can enrich legal history, bringing new elements into the debate, and, essentially, allowing new interpretations to the infinite possible legal experiences.

Understanding legal experiences as "all possible relationships with the feeling of legality (including its violations and contradictory interpretations), beyond laws and beyond possibly positivized feelings by them" ${ }^{25}$ it may be possible to increasingly include color, design, lives and paintings in these lines, so often painted in black and white, of legal history.

24 Catoni de Oliveira (2009) 367-399.

25 Silveira Siqueira (2011) 73. 


\section{Bibliographical References}

Newspapers from São Paulo and Rio de Janeiro were consulted in the Archive Edgard Leuenroth of the State University of Campinas - Unicamp. The newspapers of Minas Gerais were consulted in the newspaper library of the State Public Library of Minas Gerais "Luiz de Bessa".

Cattoni de Oliveira, Marcelo Andrade (2009), Democracia sem espera e processo de constitucionalização - Uma crítica aos discursos oficiais sobre a chamada "transição política brasileira", in: Cattoni De Oliveira, Marcelo Andrade, Felipe Machado (Coord.), Constituição e Processo: A resposta do constitucionalismo à banalização do terror, Belo Horizonte

Hespanha, António Manuel (2005), Cultura jurídica européia: Síntese de um milênio, Florianópolis: Fundação Boiteux

Hungria, Nelson (1936), Compêndio de direito penal, Rio de Janeiro: Jacyntho

Leme, Dulce Maria Pompeo de Camargo (1984), Hoje há ensaio: a greve dos ferroviários da Cia Paulista - 1906, Universidade Estadual de Campinas

Moraes, Evaristo De (1905), Apontamentos de direito operário, Rio de Janeiro: Imprensa Nacional

Nomelini, Paulo Christina Bin (2010), Mutualismo em Campinas no início do século XX: possibilidades para o estudo dos trabalhadores, in: Revista Mundos dos Trabalhadores 2, 5, agosto-dezembro, 143-173

Silveira Siqueira, Gustavo (2011), História do direito pelos movimentos sociais: cidadania, experiências e antropofagia jurídica nas estradas de ferro (Brasil, 1906), Universidade Federal de Minas Gerais, Belo Horizonte

Zambello, Marco Henrique (2005), Ferrovia e memória: Estudo sobre o trabalho e a categoria dos antigos ferroviários da Vila Industrial de Campinas, Universidade de São Paulo 


\section{Beneficencia católica, Estado municipal e infancia. Una forma de intervención pública en el ámbito privado de la familia a fines del siglo XIX*}

En los tramos finales del siglo XIX, cuando el Estado argentino alcanzaba dimensiones nacionales y se inició la consolidación de sus instituciones, se vio simultáneamente sometido a una serie de demandas sociales que fueron leídas en clave política: se entendía que estos problemas podían poner en riesgo la gobernabilidad.

Las familias de los sectores populares y en particular los niños abandonados o los que trabajaban en la vía pública, aquellos que día a día transitaban las calles de los pueblos y ciudades afectados por el desarrollo acelerado de la urbanización y el crecimiento, ${ }^{\mathbf{1}}$ eran centro de preocupación, en tanto se creía que podían devenir en delincuentes y comprometer así el futuro capital humano de la joven república.

Frente a una sociedad que se complejizaba día a día, ese Estado no podía hacer caso omiso a las múltiples preocupaciones que despertaban estas problemáticas, pero tampoco contaba aun con los medios humanos y materiales necesarios para hacerles frente. Necesitaba además, buscar una forma de intervenir para garantizar el orden, sin coartar las libertades individuales. Concordante con la lógica liberal en que se basaba ese Estado, y que preveía

* Una versión preliminar de este trabajo fue presentado en el Seminario «Derecho privado y modernización en América Latina y Europa en la primera mitad del siglo XX», organizado por el Instituto de Investigaciones de Historia del Derecho de Buenos Aires y el Max Planck Institut de Alemania. Agradezco los comentarios y sugerencias que en esa oportunidad me hicieron los Dres. Víctor Tau Anzoategui, María Rosa Pugliese, Thomas Duve, Daniela Marino, Mario Losano, Eduardo Zimmermann, Alfons Aragoneses y Agostina Gentili.

1 Si bien la mayor parte de los trabajos se refieren al impacto de la modernización y urbanización producto de la llegada de la inmigración masiva a las costas de Buenos Aires o Rosario, cabe subrayar que esos efectos se hicieron sentir también, aunque en distintas dimensiones, en pueblos y pequeñas ciudades de las provincias del interior, y especialmente en las del litoral o la región pampeana, que recibieron el mayor volumen de esa inmigración. Tal fue el caso de Azul y Tandil, localidades del centro y sur bonaerense que se analizan en este trabajo. 
la no intervención del mismo en cuestiones del ámbito privado, se creía que la respuesta a las problemáticas sociales eran obligaciones de orden moral antes que políticas.

Por ende, la solución a estas cuestiones se encontró recurriendo a viejas fórmulas de delegación de funciones asistencialistas. Las congregaciones religiosas o laicas y las mujeres dentro de ellas, desempeñaron papeles protagónicos en este sentido. A una distancia prudencial pero no desconectada con el poder de turno, se instituyeron como garantes de la estabilidad social, en tanto la fundación de asilos para huérfanos ocupó en no pocas oportunidades el centro de las actividades caritativas femeninas. A través de sus prácticas asistenciales, las damas se constituirían en protectoras de la infancia abandonada y vulnerable y, por extensión, del orden social que esos niños parecían poner en peligro.

En este trabajo pretendemos analizar el desarrollo de la política social católica, a través de la intervención practicada en el ámbito familiar orientadas a la atención de la niñez en peligro: nos referimos a los Asilos para huérfanas instalados y administrados por las Sociedades Damas de Caridad del Sagrado Corazón de Jesús, congregaciones laicas femeninas de fuerte vinculación con la Iglesia local de los pueblos analizados. ${ }^{2}$

En tal sentido, se busca en principio subrayar el carácter político que revistió el trabajo realizado por estas mujeres en el marco de un Estado que necesitó legitimar su intervención en el ámbito privado de la familia, pero sin atentar contra los derechos y libertades individuales que al mismo tiempo pretendía garantizar.

En segundo lugar, y en el marco de la multiplicación de discursos que enfatizaban la importancia de los derechos de los padres sobre sus hijos, pero también sus obligaciones para con ellos, interesa ver los modos que asumió esa intervención toda vez que se discutía no solo la necesidad de proteger a la niñez vulnerable, sino en definitiva, los alcances de la patria potestad. Si bien fue recién con la Ley Agote de 1919, cuando este régimen sufrió modificaciones en relación a lo estipulado por el Código Civil de Vélez Sarsfield, las discusiones en relación a esto alcanzan un período previo y en ellas no estuvieron ajenas las instituciones de beneficencia. ${ }^{3}$

2 La Sociedad de Azul se organizó en noviembre de 1886 y la de Tandil por su parte, en junio de 1888. En Azul el Asilo fue fundado en 1896 y en Tandil en 1897.

3 Para ello, analizaremos los Libros de Actas de las instituciones asilares que las asociaciones mencionadas organizaron en Tandil y Azul. 


\section{Las Damas de Caridad y la niñez desamparada}

En las décadas finales del siglo XIX, Tandil y Azul asistieron a cambios que transformaron a estos pueblos de campaña en pujantes ciudades al calor de la multiplicación de su población, ${ }^{4}$ momento que vería allí el nacimiento de instituciones destinadas a la contención y amparo de la infancia vulnerable, especialmente de las niñas huérfanas y abandonadas, como fueron los Asilos antes mencionados.

Como adelantamos, en el marco de construcción del Estado nacional en los últimos tramos del siglo XIX, se asistió a una proliferación discursiva en cuyo centro la familia comenzó a ser pensada en clave de estabilidad política y social: los niños se convirtieron en trascendentes ya que serían en el futuro los encargados de construir una nación moderna y civilizada, en cuanto se transformaran en ciudadanos y trabajadores. La condición de hijo, niño y alumno, resumía los atributos otorgados a la infancia, ${ }^{5}$ ó a una redefinición de los roles que a la familia le cabía respecto de ellos.

En el marco de un Estado liberal, que no preveía la intervención en el ámbito privado de la familia, estas consideraciones habilitaron sin embargo, una mayor injerencia en ella y especialmente sobre la de los sectores populares, que parecían no adecuar sus comportamientos a los ideales prescriptos. Por medio de una extrapolación del niño a la sociedad, se legitimó la intervención sobre las familias pobres, lo que conllevó siempre, decimos, a una reformulación de hecho del principio de patria potestad. Como sostiene Illanes para el caso de Chile, «El cuerpo del niño pobre será la categoría desde donde se buscará regenerar a toda la sociedad». ${ }^{6}$

En Argentina, el régimen civil de la familia, encontró sus fuentes en el Código Civil francés, la legislación castellana y el derecho canónico de la época colonial, y tal como sostiene Jaqueline Vasallo, «(...) continuó basado en la potestad y la obediencia». ${ }^{7}$ Tal es así que según el texto legal vigente en nuestro periodo de estudio, ${ }^{8}$ ésta comprendía «(...) el conjunto de los dere-

4 Azul, fue declarado ciudad en agosto de 1894. Tandil, entretanto, accedió a este estatus en 1895.

5 Zapiola (2007).

6 Illanes (2007) 120.

7 VASALlo (2008) 193-206, 204.

8 El Código Civil argentino redactado por Vélez Sarsfield, fue aprobado a libro cerrado el 29 de septiembre de 1869 mediante la ley $N^{\circ} 340$ y entró en vigencia el $1^{\circ}$ de enero de 1871 . 
chos que las leyes conceden a los padres desde la concepción de los hijos legítimos, en las personas y bienes de dichos hijos, mientras sean menores de edad y no estén emancipados». Este derecho excluía a las madres (excepto las viudas), y llevaba implícito además un conjunto de obligaciones de los hijos hacia los padres, tales como «(...) cuidarlos en su ancianidad, en el estado de demencia o enfermedad y proveer a sus necesidades en todas las circunstancias de la vida en que le sean indispensables sus auxilios», lo que no expiraba con la emancipación. ${ }^{9}$

Sin embargo, y ante la alarma que el abandono creciente de niños despertaba en la sociedad del momento, estas cuestiones se transformaron en objeto de discusión, especialmente en el caso de los derechos que la ley otorgaba a los padres cuando estos dejaban a sus hijos al cuidado de instituciones de caridad.

Particularmente sensible a estas cuestiones fue la Sociedad de Beneficencia porteña, ya que ante las situaciones de abandono y posterior reclamo por parte de padres y madres a los que debían atender en número creciente, tendieron cada vez más a interpretar estas actitudes como basadas en el aprovechamiento de éstos, antes que en necesidades genuinas. Esto las llevó a batallar largamente reclamando que los principios del Código Civil que preveían la pérdida de la Patria Potestad por el abandono de los hijos, pudieran reforzarse. ${ }^{10}$ Como sostiene Carla Villalta, «(...) la Sociedad de Beneficencia estaba empeñada en la sanción de una ley sobre la pérdida de la patria potestad». ${ }^{\mathbf{1 1}}$ Entretanto, endurecían cada vez más su postura a la hora de evaluar las solicitudes de devolución de niños efectuadas por los padres.

Las cuestiones referidas a la Patria Potestad no sufrieron modificaciones significativas hasta la sanción de la Ley Agote (o ley 10903 de Patronato de Menores) en 1919.

9 Código Civil, Libro I, Sección II, Título III: De la Patria Potestad, Arts. $1^{\circ}, 2^{\circ}$ y $3^{\circ}$, Buenos. Aires: Imprenta de La Nación Argentina 1868.

10 Esta se perdía cuando la madre viuda contraía segundas nupcias, si los padres trataban a sus hijos con excesiva dureza o le daban ejemplos inmorales, por ausencia o incapacidad mental de los padres y cuando estos abandonaron a sus hijos en la infancia. Este último punto era en el que insistían las Damas de Beneficencia, al sostener que la exposición en los Asilos debía ser causa justificada para el retiro definitivo de la Patria Potestad, al margen de las circunstancias que rodearan tal decisión. Código Civil, Libro I, Sección II, Título III: De la Patria Potestad, Arts. $44^{\circ}, 45^{\circ}, 46^{\circ}$ y $47^{\circ}$.

11 Villalta (2010) 71-93, 81.

$214 \quad$ Yolanda de Paz Trueba 
Cabe señalar que en el espacio trabajado, la preocupación en torno a la niñez desvalida era compartida por la comunidad en su conjunto, lo que decidió con frecuencia que la intervención de las Señoras que trabajaron por su protección, se diera a partir del pedido de madres o padres pero también de alguna denuncia que llegaba a ellas desde vecinos, parientes y allegados, desde que las instituciones de ambos pueblos aceptaban niñas totalmente huérfanas pero también aquellas que no lo eran, y que se admitían en calidad de pensionistas, a cambio de una mensualidad. Cabe señalar que las huérfanas también debían pagar esa cuota mensual, pero en la práctica tanto éstas como las que tenían familia, podían pagar o no, dependiendo de la situación económica de cada una, que era sopesada ante cada pedido de ingreso.

La actuación de la comunidad fue lo que determinó la suerte de una niña huérfana de padre y madre que se encontraba alojada en casa de la familia Guzmán, en Azul. En 1897, a poco de haber sido fundado el Asilo de las Damas de Caridad en el pueblo, y según comentaba la presidenta al resto de la comisión, le habían dado aviso de que era deseo de tal familia entregarla a dicha institución, cosa que fue aceptada. ${ }^{12} \mathrm{Si}$ en este caso no se hacía referencia a las razones por las que se manifestaba tal intención, frecuentemente se aludían motivos económicos, los que primaban cuando el ingreso de niñas al Asilo era pedido por los mismos padres, particularmente las madres.

Así sucedió con Teófila Delfino, cuyo caso presentó a la Comisión de Azul el cura párroco, quien señalaba que tal señora «(...) propone se le tome su hija Elvira en el Asilo pagando por pensión mensual $\$ 5$ y además que se compromete a ayudar en los trabajos de dicho establecimiento, siempre que le sea posible». Las Damas de Caridad decidieron aceptarla «(...) hasta tanto esa señora se halle en condiciones más favorables para sostener a su hija». ${ }^{13} \mathrm{Si}$ bien el reglamento del Asilo de Azul establecía que las niñas debían ingresar y permanecer en él hasta su mayoría de edad que se cumplía a los catorce años, ${ }^{14}$ y aunque el no cumplimiento del tiempo esperable de estadía fue

12 Archivo Sagrado Corazón de Jesús de Azul (ASCJA), Actas, 3-6-1897.

13 ASCJA, Actas, 2-8-1897.

14 En Tandil por su parte, el reglamento interno establecía que la permanencia debía ser de al menos cuatro años, tiempo necesario para transmitir un mínimo de educación a las niñas que ingresaban. La mayoría de edad era el límite permitido para permanecer en el Asilo, cuando la estadía (especialmente de aquellas que no tenían familia o tutor), superaba ese mínimo de tiempo exigido.Según lo establecía el Código Civil, se consideraban menores de edad los individuos de uno y otro sexo que no llegasen a 22 años cumplidos. Los 
esgrimido con frecuencia para denegar la entrega de alguna niña, no fue así en el caso de la hija de Teófila (quien de antemano era ingresada sólo por un tiempo), flexibilidad que podría explicarse a causa de las dificultades económicas por las que usualmente atravesaba el Asilo para mantenerse. ${ }^{\mathbf{1 5}}$

Dado que las demandas de internación superaban las posibilidades de los Establecimientos, esta situación de escasez se tornaría endémica en ambas instituciones. La fragilidad económica era moneda corriente en ellas, revistiendo a veces mayor gravedad en el caso de Tandil, ya que no contaba (como sí el de Azul), con la colaboración municipal. Es decir que al ser costeado enteramente por la caridad privada, los apremios eran aún más graves. Tal era así que en 1899 , la presidenta comentaba a la comisión que «(...) en vistas del precario estado en que se encuentra el Asilo creía muy oportuno pasar nota a las personas capitalistas de esta población pidiendo ayuda para sostener tan humanitario estabelecimiento (...)», lo que aceptaron los miembros presentes. La recorrida de la ciudad en búsqueda de nuevos socios así como el incremento de la cuota mensual a los ya existentes, fueron dos medidas adicionales tomadas en la misma ocasión. ${ }^{16}$ Tiempo después la situación se agudizó, a tal punto que se llegó a la resolución de pedir un préstamo al Banco Nación para abonar una deuda de panadería y carnicería, dada la demora por la que se atravesaba en la recepción de las subvenciones del Gobierno de la provincia y de la Lotería Nacional. ${ }^{\mathbf{1 7}}$

Las inseguridades económicas institucionales se acentuaban cuando el pedido de ingreso de niñas a los Asilos se daba con frecuencia como señalamos, por la imposibilidad económica de las familias de hacerse cargo de las pequeñas, lo que redundaba en las dificultades para aportar la cuota reque-

menores de 14 años, por su parte, eran considerados impúberes. Código Civil, Libro I, Sección I, Título IX: De los menores, Arts. $1^{\circ}$ y $2^{\circ}$.

15 Para el sostenimiento económico, contaban con la imprescindible colaboración privada de la comunidad en general, la organización de bazares, beneficios, rifas, etc. y, desde 1896, un porcentaje de la Lotería Nacional. Archivo Histórico Municipal de Tandil (AHMT), Correspondencia, Abril 27 de 1896. Además, contaban con los recursos obtenidos por medio de las mensualidades de las niñas que eran alojadas como pensionistas y no sólo huérfanas y con una subvención que desde 1895, destinaba el Gobierno Provincial. Registro Oficial de la Provincia de Buenos Aires, Mayo 7 de 1895, La Plata: Talleres de Publicaciones del Museo 1895.

16 Archivo Sagrado Corazón de Tandil (ASCJT), Actas, 12-11-1899.

17 ASCJT, Actas, 24-3-1901. El Banco no prestaba dinero a entidades, por lo cual se decidió solicitarlo a nombre de la presidenta.

216 Yolanda de Paz Trueba 
rida al Establecimiento, como en el mencionado caso de Teófila, en el que se solicitaba la internación a cambio de una mensualidad inferior a la establecida por reglamento (fijada en $\$ 12$ mensuales). ${ }^{18}$ Cuando la indigencia familiar o la completa orfandad así lo ameritaban, se permitía el ingreso y permanencia de forma gratuita. De hecho y como lo especificaba el Acta de inauguración del Asilo de Tandil, se trataba de una institución para albergar «niños huérfanos y pobres». ${ }^{19}$

La pobreza absoluta definió favorablemente en 1899 el caso de dos niñas de Azul que las damas de esa ciudad decidieron tomar en guarda hasta la mayoría de edad, hijas «(...) del señor Domingo Romeo por encontrarse completamente en la indigencia». ${ }^{\mathbf{2 0}}$ La pobreza manifiesta de Romeo, justificó sobradamente para las Damas de Azul la solicitud del padre en un momento en que las ya comentadas discusiones en torno al alcance de la Patria Potestad estaban a la orden del día en un contexto más amplio. Tal cariz iban tomando estas concepciones a nivel nacional, que en los años previos a la sanción de la nueva Ley de Patronato, se tornó frecuente deslegitimar y hasta desconocer las situaciones personales que llevaba a madres y padres a acudir en busca de ayuda, reprobando el hecho de dejar a sus hijos al cuidado de alguna institución de beneficencia, al englobar en el concepto de abandono tanto a aquellos que dejaban a sus niños llevados por su extrema necesidad, como aquellos que lo hacían casi de un modo estratégico.

Claro que tal discurso no era uniforme y así como muchos estigmatizaban a las madres «desnaturalizadas» que abandonaban a sus hijos, otros hacían hincapié en el sacrificio que representaba para ellas dejarlos ante la necesidad de trabajar para su propio sostenimiento y probablemente por el de otros hijos a quienes debían alimentar y vestir. Como sostenía Meyer Arana en 1904, la pobreza era la causa del deambular de niños por las calles, y no el descuido de los padres y madres de las acciones de sus hijos. Simplemente estaban atados a un destino familiar difícil de torcer mientras su situación económica los obligara a trabajar largas horas fuera del hogar durante el día. Así, decía que «(...) Esa madre, dicha con ligereza madre desnaturalizada

18 En Tandil, la cuota mensual era de entre $\$ 12$ y $\$ 15$, según las circunstancias de cada niña, como se explicaba. ASCJT, Actas, 10-11-1901. En 1903, decidieron que la cuota se estipulara en $\$ 15$ y no en $\$ 12$.

19 ASCJT, Actas, 4-9-1897.

20 ASCJA, Actas, 20-11-1899. 
(olvidándose que el sufrimiento de sus hijos es un suplicio para toda madre), no puede retener a aquellos junto a sí (...) porque no dispone de sitio en que colocarlos ni de juguetes para distraerlos». ${ }^{\mathbf{2 1}}$

Ahora bien, si no se discutía desde este punto de vista el derecho al ejercicio de la patria potestad, el centro de la discusión pasaba, tal como años antes había postulado el mismo autor, por el papel que cabía al Estado en pos de solucionar los males sociales que causaban esa pobreza. Así, tal como afirmaba, la asistencia pública practicada por el Estado y la beneficencia privada llevada adelante por particulares, debían complementarse para combatir la miseria. Pero esa complementariedad debía tener ciertos límites ya que, afirmaba, «(...) al Estado solo incumbe la realización de limitados actos de asistencia (...) no puede llevar su acción tan lejos que ultrapase sus propios límites e invada la acción individual». ${ }^{22}$ Para este autor estaba claro que el Estado debía ser regulador de acciones sociales, mas no tomar la iniciativa. En un contexto plagado de discursos encontrados, no todos acordaban con la necesidad de traspasar los umbrales del hogar.

\section{Las Damas locales y las estrategias de intervención}

Al margen de las discusiones comentadas y de las posturas cada vez más rígidas tomadas por las emblemáticas matronas de la Sociedad porteña, hallamos algunos matices en los casos locales estudiados. El acercamiento de la comunidad y con frecuencia de los progenitores en búsqueda de ayuda, aun no era aquí sometido a ninguna discusión, cuando las causas aludidas (siendo las más frecuentes la pobreza, la enfermedad o la viudez), se consideraban ciertas o suficientes para atender a tales demandas. Era en cambio más usual que en ciertas ocasiones se negara el ingreso de niñas amparándose en el reglamento en lo relativo a la edad de admisión mínima o máxima estipulada, y lo cierto es que la fluidez en el ingreso y egreso prima a la hora de hacer una evaluación de conjunto. ${ }^{23}$

Ahora bien, a media que transcurría el tiempo de haberse fundado el Asilo, la problemática del reclamo de las niñas se fue colando tímidamente en las discusiones de la Comisión hasta tomar una forma más definida y

21 Meyer Arana (1904) 11.

22 Meyer Arana (1901) 61.

23 Sobre estos casos ver entre otros ASCJT, Actas, 24-3-1901; 26-10-1902 y 3-1-1905.

218 Yolanda de Paz Trueba 
llegar a declarar abiertamente la intención de ir más allá en la política de intervención que estaban llevando a cabo.

Si tal injerencia era en principio pedida por una amplia gama de actores sociales que incluía a la misma familia, al hacerse cargo de la crianza y educación, las Damas se arrogaron también el derecho a tomar sus propias determinaciones cuando la situación lo requería, como podía ser si se suscitaban problemas en relación a la conducta que observaban las internas durante su estadía en el Asilo.

Cuando alguna situación problemática en relación a este tema se presentaba, un principio de solución se hallaba en el envío de la niña o niñas a alguna institución de Buenos Aires o La Plata. Así, las Damas de Azul manifestaban en 1899 haber mandado dos niñas a la Correccional por el término de seis meses. ${ }^{\mathbf{2 4}}$ También la Santa Casa de Ejercicios era un destino posible para aquellas pequeñas que vulneraban las reglas institucionales, como fue el caso de Nieves Muñoz de Azul. Pero si en el primer caso, se establecía un límite de tiempo para el retorno al pueblo, el destino de Nieves en cambio, fue diferente. Meses más tarde, la Superiora del Asilo enviaba una nota a las Damas pidiendo autorización para entregar a la citada menor, que aun se encontraba en la Casa de Ejercicios, a una hermana que vivía en Entre Ríos, permiso que se le concedió. ${ }^{25}$

A la salida del Asilo y posterior ingreso a otra institución que pudiera contener esos problemas de conducta y eventualmente corregirlos, se ofrecía como alternativa la colocación de las niñas y jóvenes que presentaban este tipo de inconvenientes, con alguna familia del pueblo. Así lo proponía la presidenta de la Sociedad de Tandil en relación a Teresa Montenegro ya que, según explicaba, «(...) las Hermanas la tienen siempre separada de las otras huérfanas a causa de su mal carácter (...)». Además, como subrayaban, se trataba de una niña completamente huérfana, que nadie reclamaría ya que la persona que hacía tres años la había entregado al Asilo por encontrarse enferma, había ya fallecido. ${ }^{26}$

Si bien el reclamo de las niñas ingresadas tenía antecedentes desde los primeros años de funcionamiento de la Institución, fue recién en 1901 cuando nos encontramos con indicios de que esto comenzó a percibirse

24 ASCJA, Actas, 10-9-1899.

25 ASCJA, Actas, 24-11-1901 y 22-3-1902.

26 ASCJT, Actas, 24-3-1901. 
como problemático, desde que la colocación en casas de familia implicaba no solo una solución momentánea, sino también la posibilidad de que la chica encontrara una ubicación más estable a posteriori, preocupación que, como veremos, se fue haciendo más apremiante con el paso de los años. En este sentido, la no injerencia de la familia en el destino que las Damas proyectaban para cada niña era una condición cada vez más necesaria. El proyecto social encarado por las Damas de Caridad proponía la formación de un perfil de mujer que lograra insertarse en la sociedad tras cumplirse el tiempo de estadía en el Asilo, a través del desarrollo de ciertas habilidades y conductas. Desde su óptica, tal inserción social se veía facilitada cuando la estadía con familias decentes podía prolongarse tras la salida del Asilo, lo que las Señoras creían que se vería obstaculiza cuando alguien solicitaba su restitución.

Si la protección de las internas, ameritaba a veces la salida de alguna de ellas del Asilo, no siempre era tal el deseo de las Damas, quienes con frecuencia consideraban más conveniente la permanencia de ciertas niñas en sus Establecimientos, aun teniendo que poner en discusión los reclamos de los mismos padres o madres que otrora los habían colocado allí. Así sucedió (a pesar de la decisión final), con Ema y Clara Romeo. Tras ser pedidas por el padre, se generó un debate entre las socias al respecto, ya que Romeo había firmado un documento comprometiéndose a no pedir a sus niñas hasta tanto cumplieran la mayoría de edad, lo que se estipulaba para todos los casos. Si bien el padre era claro depositario de la patria potestad y tenía a su favor los derechos que la ley le otorgaba, el pedido generó dudas y diferentes posturas, que sin embargo se resolvieron a su favor. Tras el intercambio de ideas, se aceptó finalmente su pedido. ${ }^{27}$

En Tandil, el reglamento estipulaba como señalamos, la permanencia de las niñas por un mínimo de cuatro años, pero como en el caso de Azul, esto podía variar de acuerdo a las condiciones particulares de cada interna y su situación familiar. Si bien como plantea Villalta, la tendencia general parecía ir virando hacia la condena del abandono mas allá de las circunstancias que lo rodeaban, en espacios más pequeños como los estudiados aquí, esto nunca dejó de ser considerado; probablemente esas condiciones particulares eran bien conocidas por las socias.

27 ASCJA, Actas, 20-1-1900.

$220 \quad$ Yolanda de Paz Trueba 
Cuando Lidia Avellaneda fue pedida por su madre en 1901, faltaban unos meses para que se cumpliera el tiempo de estadía. Sin embargo, lo que pareció decidir su suerte no tenía que ver con esto, ya que, como se explicaba «(...) acordaron no acceder a la solicitud y aun no entregarla cumplido su tiempo que todas tenían malos informes del genero de vida que observaba la madre, muy lejos de asegurar conducta moral para su hija». ${ }^{28}$ Similar resolución se tomó tiempo después con Isabel Mercado, quien pedía retirar a sus hijas del Asilo, a pesar de no haberse cumplido el tiempo estipulado de estadía. Pero teniendo en cuenta los datos que las Damas decían tener sobre la madre, decidieron dar intervención al Defensor de Menores, coincidiendo en que «(...) las damas en mayoría fueron de opinión de no entregar las niñas hasta que cumplieran el tiempo que expresa el Regalmento». ${ }^{29}$ Años más tarde, ante un nuevo pedido de la madre, se decidió acceder a entregarle a una de las pequeñas, de nombre Mercedes, aunque aún no había cumplido el tiempo. Según se decía, la mujer «(...) se había presentado pidiendo le fuera entregada la citada menor por serle muy necesaria su ayuda». ${ }^{\mathbf{3 0}} \mathrm{Nada}$ se mencionaba sobre la otra niña, ni sobre los motivos del cambio de opinión.

Tampoco se aceptó la entrega en el caso de Magdalena Sedeillan que era pedida por un tal Raymon (con quien se desconoce la relación), tras haber recibido «(...) orden superior del Señor Defensor de Niños». ${ }^{31}$ Meses más tarde, acordaron por orden del Defensor General de Menores de La Plata, cuya nota leyeron en reunión, conceder a Raymon ver a la mencionada menor tan solo los días de visita. ${ }^{32}$

Así, el carácter de la intervención de las Damas en función de proteger el destino de las menores, tomaba otros ribetes cuando se hacía caso omiso de la voluntad de la familia. La mayor parte de las veces, la situación se resolvía informalmente, llegando a un acuerdo entre las Damas y quienes habían solicitado su asistencia. Estas resoluciones sin embargo, no traslucen largos o conflictivos litigios. Los padres y sobre todo las madres parecían aceptar las decisiones que las Damas tomaban en relación al destino de sus hijas. En el caso de las instituciones de beneficencia estudiadas a nivel local, no encon-

28 ASCJT, Actas, 24-3-1901.

29 ASCJT, Actas, 26-10-1902.

30 ASCJT, Actas, 3-1-1905.

31 ASCJA, Actas, 5-8-1900.

32 ASCJA, Actas, 27-10-1900. 
tramos posturas duras a la hora de juzgar a los progenitores que recurrían a ellas en búsqueda de socorro y en general, de no mediar alguna consideración moral sobre la conducta de la madre, la devolución se efectuaba sin dilaciones.

Si bien como plantea Villalta en su estudio para la Capital, frente a la negativa de las Damas de devolver a sus hijos, los padres podían establecer un litigio judicial y «En la década del '20 las demandas judiciales sobre entrega de menores poseían extensos antecedentes (...)», ${ }^{33}$ en los casos locales estudiados no encontramos respuestas judiciales por parte de los padres cuando se encontraban con negativas de las Damas a los pedidos de restitución de sus hijos. ${ }^{34}$ Las situaciones litigiosas se daban en cambio, entre los padres y los Defensores de Menores locales, tal como lo reflejan los registros de esas dependencias. Cuando algún desacuerdo se generaba entre los padres y aquellos, la familia no dudaba en recurrir al Defensor General de La Plata si creían que la autoridad local había actuado incorrectamente. Sin embargo, esta no fue la actitud que asumieron en el caso de desacuerdos mantenidos por motivos similares con las Damas de Caridad. Todo hace pensar que el prestigio esgrimido por las Señoras y que se fue incrementado con el correr de los años al calor de la consolidación de sus instituciones, sirvió para ejercer un tipo de intervención que lejos de ser pensada como impuesta, era compartida por la comunidad en tanto era ella misma quien se acercaba a pedir ayuda, aun a cuenta de coartar su propia libertad de decisión sobre el futuro de los hijos que por derecho le cabía solo a la familia. Al aceptar de mejor talante sus decisiones que las impuestas por el Defensor de turno, ponían de manifiesto una aceptación que iba más allá del simple acatamiento de normas y reglas.

Haber confiado el cuidado de las niñas a una Institución ajena a la familia, habilitó en más de una oportunidad diferentes maneras de intervención, cuyo carácter fue sufriendo algunos cambios conforme los Asilos se consolidaban. Así, en 1901 como adelantamos, la presidenta de la Sociedad de Tandil, comenzaba a reflejar la problemática que se les presentaba, ante los reclamos que efectuaban las madres de aquellas niñas que tiempo antes habían dejado a su cuidado. Según señalaba, éstas solían apurarse para recla-

33 Villalta (2010) 83.

34 Cabe señalar que una excepción lo representa el caso de María Francisca Boulanger, al que nos hemos referido ampliamente en De Paz Trueba (2010).

222 Yolanda de Paz Trueba 
mar a sus hijas cuando se cumplía el tiempo de cuatro años y, aunque en ese momento tenían varias niñas en condición de regresar con la familia, decidieron no permitirlo y darle participación al Defensor, ya que «(...) como la Sociedad hubiese recibido días antes una nota del Juez de Menores en la que pedía su interbención (sic) en la salida de las niñas (...)», se acordó proceder de tal manera. ${ }^{35}$ La intervención del Defensor legitimaba legalmente lo que sería una tendencia de allí en más, y que partía siempre de decisiones tomadas por ellas.

A medida que el camino de asistencia emprendido por las Damas se iba consolidando y se presentaban nuevos desafíos, comenzaron a barajarse otras posibilidades de cara a la salvaguarda de la infancia y de proteger no solo a quienes tenían edad para permanecer en el internado, sino también de hacer extensiva esa protección a quienes estaban en condiciones de salir de él. Así, cuando las instituciones tenían ya varios años en funciones, y comenzaron a darse casos de niñas que completaban su tiempo de estadía, las Damas de Tandil proponían tener derecho a «(...) disponer de ellas una vez que concluyeran el tiempo porque fueron puestas en el Asilo», sugerencia hecha por la señora de Suárez Martínez y aceptado por las demás. ${ }^{36}$ Meses más tarde, y siguiendo en el empeño de disponer del futuro de las asiladas y pensionistas, las señoras de Tandil acentuaban la importancia de dejarlo establecido en el Reglamento interno e insistían en acelerar su modificación, de modo de evitar reclamos posteriores, en un marco donde si bien estaba sometido a discusión, ninguna ley se había sancionado aun como modificatoria de las disposiciones del Código Civil en materia de Patria Potestad. En este tenor, la presidenta hacía hincapié en la importancia de reformar el Reglamento interno

«(...) cuanto antes para que las madres al colocar una niña en el Asilo supiesen que una vez terminado el tiempo porque fue puesta en el Establecimiento pudiera la sociedad colocarlo en cualquier casa de familia (...) y no sucediese como con las que acababan de salir del Asilo que las madres se han resistido a que fuesen colocadas y solo ellas han disfrutado de la enseñanza dada a sus hijas.» ${ }^{37}$

Todo parece indicar que efectivamente procedieron de tal manera ya que en 1903, se comentaba en relación a la mensualidad, que quien no pudiera

35 ASCJT, Actas, 6-6-1901.

36 ASCJT, Actas, 6-6-1901.

37 ASCJT, Actas, 10-11-1901. 
pagarla se atuviera al Reglamento, es decir, cumplida la estadía, las niñas debían quedar a disposición de las Damas para que las ubicaran en casas de familia.

Era la comunidad la que sostenía la educación de esas niñas, y la intención al disponer de ellas tras completar su formación, era un modo de devolver a ésta lo que había hecho por ellas, haciendo de la intervención en el ámbito privado de la familia una cuestión pública: era público el interés por la salvaguarda de la infancia ya que era la sociedad a quien se protegía mas allá de la necesidad de los padres.

Pero además, la permanencia con estas familias podía asegurar un futuro menos incierto que el que prometía el regreso con la propia, previniendo así males futuros, lo que redundaba en el carácter público que tenía la intervención en el ámbito privado, a diferencia de los reclamos de los padres que solo respondían, como sostiene Villalta, a intereses estrictamente privados: «(...) la permanencia de los niños en esas familias honestas no constituía un motivo privado, como sí lo eran los reclamos efectuados por sus padres, sino un bien para la comunidad». ${ }^{38}$

\section{Conclusiones}

Frente a las modificaciones que sufrieron a fines el siglo XIX las concepciones sobre la infancia, el Estado se vio ante la necesidad de practicar alguna forma de intervención en aquellas familias que demostraban no poder brindar a sus hijos un adecuado marco de contención.

No obstante, la ideología liberal que amparaba a ese Estado, hacía incompatible esta necesidad con la injerencia concreta en el entorno familiar. En este contexto, tomó otros ribetes la práctica de la beneficencia, y especialmente aquella orientada a la infancia desvalida, como la estudiada en las páginas precedentes.

Como vimos, las acciones de las Sociedades Damas de Caridad de Azul y Tandil alcanzaron preeminencia y si la intervención en el ámbito familiar podía partir desde una incitativa de la comunidad, en no pocas ocasiones y como se desprende del análisis de los documentos institucionales, se efectivizaba a raíz de un pedido de ayuda de la misma familia. 
Tal injerencia, consentida por los actores sociales que acudían solicitando asistencia, conllevó en no pocas oportunidades, a un atentado al principio de patria potestad, sobre todo cuando las instituciones locales iban consolidando su modelo asistencial y se encontraban con nuevos desafíos a enfrentar, como eran los pedidos de restitución de las niñas realizados por las familias que tiempo antes habían colocado bajo su guarda y protección.

Si bien todo parece indicar que la posición no fue en nuestro espacio de estudio tan dura como la que tomaron las Damas de Beneficencia de la Capital quienes, como plantea Carla Villalta, avalaban la pérdida de la patria potestad de los progenitores tras el abandono, creyéndose llamadas a salvar definitivamente a los menores de padres que no cumplían los requisitos morales para enfrentar sus obligaciones, ${ }^{39}$ a medida que transcurrían los años, estas posiciones se fueron endureciendo, sobre todo en el caso de Tandil. A principios del siglo XX, las Damas de este pueblo manifestaban su intención de poder decidir sobre el futuro de las asiladas y pensionistas, una vez cumplido el tiempo de estadía en el Asilo. Si el regreso con la familia, en general no era sometido a discusión, las Señoras comenzaron a delinear un pensamiento que las llevó a plantear la necesidad de reformar el Reglamento Interno de modo de no permitir tal restitución.

Como lo dejan entrever en sus Actas, la posibilidad de colocarlas con familias del pueblo a largo plazo, muestran un viraje en la forma de encarar el asistencialismo y su misión para con las niñas del pueblo, desde que poco a poco fueron formándose una idea acerca del mejor futuro posible para ellas, que en muchos casos no lo concebían al lado de la familia propia.

\section{Fuentes y Bibliografía}

De Paz Trueba, Yolanda (2010), Mujeres y esfera pública: la campaña bonaerense entre 1880 y 1910, Rosario: Prohistoria Ediciones

Illanes, M. Angélica (2007), Cuerpo y Sangre de la política. La construcción histórica de las Visitadoras Sociales. Chile, 1887-1940, Santiago de Chile: LOM Ediciones

Meyer Arana, Alberto (1901), Apuntes sobre la beneficencia, Buenos Aires: Imprenta de M. Biedma e Hijo 
Meyer Arana, Alberto (1904b), Por el niño pobre, Buenos Aires

Vasallo, Jaqueline (2008), La construcción de la feminidad y la masculinidad en la doctrina jurídica y su impacto en la legislación argentina del siglo XIX, en: Ghirardi, Mónica (comp.), Familias iberoamericanas ayer y hoy. Una mirada interdisciplinaria, Córdoba: Ferreyra Editor, 193-206

Vélez Sarsfield, Dalmacio (1868), Código civil, Libro I, Buenos Aires: Imprenta de La Nación Argentina

Villalta, Carla (2010), La conformación de una matriz interpretativa. La definición jurídica del abandono y la pérdida de la patria potestad, en: Lionetti, Lucía, Lionetti, Daniel Miguez (comps.), Las Infancias en la Historia Argentina. Intersecciones entre Prácticas, Discursos e Instituciones (1890-1960), Rosario: Prohistoria, 71-93

Zapiola, Carolina (2007), La invención del menor: representaciones, discursos y políticas de menores en la ciudad de Buenos Aires, 1882-1921, Buenos Aires: UNSAM

\section{Listado de abreviaciones}

Archivo Sagrado Corazón de Jesús de Azul (ASCJA)

Archivo Histórico Municipal de Tandil (AHMT)

Archivo Sagrado Corazón de Tandil (ASCJT) 
Agostina Gentili

\section{Un fuero híbrido}

Juzgados de menores, precedentes y prácticas en Córdoba, Argentina, primera mitad del siglo XX

A fines del siglo XIX y principios del XX juristas e intelectuales argentinos bregaban por la creación de leyes, instituciones y juzgados especiales para menores. Se inspiraban en experiencias europeas y estadounidenses que conocían a través de viajes y publicaciones, difundían sus ideas en encuentros académicos con eco en la prensa, y algunos arribaban a cargos públicos de importancia en la materia. Desde sus perspectivas, esos niños y adolescentes que deambulaban en las calles, eran alojados en comisarías, o carecían de hogares «adecuados», merecían una intervención estatal específica, diferente a la destinada a quienes se ajustaban a los modelos de infancia y adolescencia socialmente deseados. Peligrosos o en peligro, los menores fueron - y aún son a pesar de los intentos por revertirlo - un sujeto social particular al que se destinaron acciones también particulares.

A partir de piezas normativas de origen diverso, como proyectos parlamentarios, leyes y decretos, y resoluciones de dependencias públicas, este ensayo propone un análisis de las instituciones cordobesas que entre la década de 1930 y los primeros años de 1960 fueron destinadas a la infancia abandonada y delincuente, como se la llamaba. Esta reconstrucción, valiéndose también del aporte de otros investigadores, pone en relación la experiencia provincial y nacional de las políticas destinadas a esos niños y jóvenes, lo que aquí se designa como minoridad, prestando particular atención al espacio otorgado a la magistratura de menores durante ese trayecto. A partir de ese diálogo, se sostiene que, más allá de los ritmos impuestos por las coyunturas políticas de la provincia y ciertos diagramas institucionales particulares, esas trayectorias abrevaron en las mismas ideas y lineamientos.

La historiografía no ofrece aún un análisis de los precedentes de la conformación del fuero de menores en la provincia de Córdoba, pero sí algunas coordenadas que lo hacen posible. María José Ortiz Bergia, en el marco de su investigación sobre políticas sociales durante los años '30, ofrece un análisis 
específico de las políticas destinadas a la minoridad en ese período, en el que sostiene que el Estado provincial abandona progresivamente su papel subsidiario para encarar uno más activo. Su trabajo brinda una reconstrucción de las principales realizaciones institucionales, centradas en la inauguración de establecimientos estatales. ${ }^{\mathbf{1}}$ El estudio de Marta Philp sobre las concepciones de Estado y las políticas sociales de los gobiernos radicales entre 1936-1943, y su comparación con las modalidades de los gobiernos peronistas, caracteriza la complicada relación que Córdoba mantuviera con el gobierno central en aquellos años. ${ }^{2}$

Para el ámbito nacional Carolina Zapiola ofrece una lectura sugerente sobre la Ley Nacional de Patronato, considerada un hito de la historia de las infancias. Comparando su contenido con el contexto intelectual y político, demuestra que sólo cuando los menores se volvieron un problema político y no únicamente social a los ojos de las élites dirigentes, dada la conflictividad social en aumento y a poco de haber tenido lugar en Buenos Aires una huelga obrera férreamente reprimida que pasó a la historia como la Semana Trágica, se logró el consenso necesario para la sanción de una ley que llevaba ya varias presentaciones parlamentarias truncas, y que tuvo un carácter más conservador de lo que tiende a afirmarse. ${ }^{3}$

Leandro Stagno ofrece un análisis de las ideas de juristas y especialistas en minoridad a nivel nacional, afirmando que en sus demandas de los años '20 se fundamentaron las concreciones institucionales de la década siguiente en la provincia más importante del país, Buenos Aires. A este autor debemos el único análisis por ahora existente sobre la constitución del fuero de menores en aquel territorio, medida impulsada por aquellos especialistas. ${ }^{4}$

La presentación de un modelo de intervención sobre la población más joven que fuera articulado a nivel nacional y en la provincia de Buenos Aires, dará paso después a la reconstrucción de las iniciativas y concreciones en la provincia de Córdoba, poniendo de relieve una trayectoria desfasada en materia de minoridad. A continuación, el análisis de expedientes prevencionales permitirá dar cuenta de las prácticas del fuero de menores cordobés hacia los años sesenta, período fundacional de esta magistratura especial.

1 Ortiz Bergia (2009a y b).

2 Philp (1998).

3 Zapiola (2010).

4 STANGo (2010). Agradezco al autor por hacerme llegar este trabajo y por su lectura generosa y atenta de un texto preliminar al que aquí se presenta. 


\section{Un modelo de intervención}

En 1919 se sanciona la Ley Nacional de Patronato de Menores. Este instrumento redefinió la patria potestad, habilitando la posibilidad de que los jueces suspendieran o quitaran su ejercicio a padres que «abandonaran» a sus hijos, cometieran delitos contra ellos o los pusieran en "peligro material o moral». Otorgó a los establecimientos de beneficencia que así lo reclamaban, la tutela definitiva de los niños que les fueran confiados por sus padres, tutores o guardadores. Sentó, por último, las bases de un proceso penal diferente para menores de edad. Fue, para Carolina Zapiola, que investigó la coyuntura específica en que esa ley se sancionó, menos rupturista de lo que tiende a pensarse. Refrendó prácticas tradicionales de vinculación de las instituciones de beneficencia con la población más joven, como las colocaciones de niños y los reformatorios, y contradijo las propuestas que se esgrimían entre científicos y juristas nacionales e internacionales, que reclamaban la instauración de juzgados de menores y establecimientos estatales de educación e internación. ${ }^{5}$

Esos establecimientos y aquel fuero se organizaron en la provincia de Buenos Aires durante los años '30. Leandro Stagno investigó los discursos y las iniciativas de los expertos jurídicos y científicos de la minoridad en Buenos Aires, reconociendo esa década como un período de difusión y consolidación de una manera de concebir la delincuencia infantil iniciada una década antes, y consagrada por la Ley Nacional de Patronato. Este ideario proclamaba un ideal punitivo basado en la necesidad de reemplazar prácticas represivas por educación, y de conocer los antecedentes familiares y personales del menor. En ámbitos intelectuales, a nivel nacional bregaban por la organización de un sistema legal exclusivo para menores, con leyes acordes a sus necesidades y a las prerrogativas internacionales, espacios de retención propios que alejaran al menor de los ámbitos policiales y judiciales de mayores, y técnicas de evaluación específicas de la situación personal y familiar del niño. Las propuestas de los expertos, algunos convertidos en funcionarios de minoridad en Buenos Aires, acogían referencias internacionales - Francia, Inglaterra y Estados Unidos eran ejemplos vanguardistas a seguir - y ensayaban métodos de estudio y clasificación de menores, reclamando una especialización médico-psicológica de los agentes judiciales. 
Según Stagno, fueron esas concepciones punitivas las que fundamentaron las políticas bonaerenses de minoridad de los años '30, marcándoles un rumbo preciso. ${ }^{6}$ En ellas se basó la organización de la Colonia Hogar Ricardo Gutiérrez bajo un sistema que se decía propiciaba una socialización acorde con la inculcación de un sistema de valores y el control de la conducta, previendo una organización de los espacios (hogares) bajo criterios esperados para una casa familiar, y con una educación basada en la enseñanza de oficios. ${ }^{7}$ En aquellas ideas se fundamentó también la constitución del fuero de menores en la provincia de Buenos Aires; el procedimiento y los conocimientos requeridos de los agentes judiciales daban cuenta, para Stagno, de la pervivencia de las mismas concepciones del castigo: el énfasis del procedimiento en conocer las conductas del menor y su familia, y la fundamentación de las resoluciones judiciales en informes ambientales y médico-psicológicos. ${ }^{8}$ Así, en la lectura que el autor propone de la difusión de las ideas y su concreción en políticas públicas, la década de 1930 tuvo en Buenos Aires una identidad propia en materia de infancia y juventud, signada por la centralización pronunciada y la profesionalización de las acciones estatales que buscaban generar condiciones materiales y morales que fortalecieran el llamado binomio madre-hijo. En 1931, el gobierno de Uriburu creó el Patronato Nacional de Menores para centralizar las políticas de minoridad ante la falta de articulación entre establecimientos públicos y privados de internación, organizados bajo regímenes que contrariaban las prescripciones de los expertos. Vinculado al Ministerio de Justicia e Instrucción Pública, debía supervisar y administrar esos institutos y coordinar las acciones de jueces y defensores de menores, patronatos provinciales e instituciones privadas. ${ }^{9}$ En 1938, con el apoyo del gobernador Fresco, la provincia de Buenos Aires organiza el patronato a partir de la creación de un tribunal de menores (el primero en el país) y una Dirección General de Protección a la Infancia, institución encargada de coordinar la acción de los establecimientos públicos y privados de tutela, definir el tipo de educación impartida y ejercer la "policía de la infancia». ${ }^{\mathbf{1 0}}$

6 Stango (2010) 1-47.

7 Ibidem 48-54.

8 Ibídem 40-62.

9 Ibidem 40.

10 Ibídem 63-67 y 75-79. 
En 1954 se sanciona a nivel nacional un nuevo procedimiento penal para menores de edad: debía tomarse conocimiento personal y directo del menor y sus padres o responsables, y estudiarse su personalidad, condiciones familiares y ambientales. La internación en un «instituto adecuado» continuaba siendo un destino posible, pero también se prescribía la posibilidad de que el menor quedara a cargo de los padres o de otros responsables, «libremente o bajo el régimen de libertad vigilada». ${ }^{\mathbf{1 1}}$ Estas actuaciones no diferían de aquéllas que Leandro Stagno describiera como prácticas del juzgado de menores de la provincia de Buenos Aires al inicio de 1940, ${ }^{\mathbf{1 2}}$ pudiendo sostenerse que la nueva legislación estaría reafirmando prácticas y nociones ya existentes, sin proponer modificaciones sustanciales.

Entre 1919 y mediados de 1950 encontramos a nivel nacional y en la provincia de Buenos Aires los componentes de una estructura institucional de intervención de largo alcance. Una ley marco que habilitaba al Estado, a través de los jueces, a quitar o suspender el ejercicio de la patria potestad a los padres considerados irresponsables. Establecimientos estatales de internación y educación de menores organizados bajo la forma de colonias-hogares, un intento por combinar la vida hogareña, la escolarización y el trabajo rural como terapéutica para quienes eran considerados corregibles. Un fuero específico para menores y un organismo administrativo encargado de centralizar la labor de los establecimientos públicos y privados de acogida y reclusión. Un régimen penal especial para menores de edad que revalida las prácticas que el fuero de menores venía desplegando desde su instauración. En la provincia de Córdoba, como intentan mostrar las páginas siguientes, existen indicios de la legitimidad de esas ideas, pero la organización institucional de la minoridad, y del fuero específico, presentan un recorrido distinto.

\section{Una trayectoria desfasada}

La política de minoridad de los gobiernos provinciales durante la década del '30 se caracterizó, según Ortiz Bergia, por el abandono progresivo del papel subsidiario del Estado en la materia y su consiguiente avance sobre terrenos que estaban tradicionalmente a cargo de la sociedad civil. Al iniciarse aquella década, el Estado provincial contaba con dos instituciones asilares: el Buen

11 Modificaciones al régimen de menores y bien de familia (1956).

12 Stango (2010) 80-87. 
Pastor, esto es, la cárcel de mujeres para las jóvenes, y el Asilo de Menores Varones, en la Cárcel de Encausados. Una decena de establecimientos estaba a cargo de congregaciones religiosas y sociedades de beneficencia, que se financiaban, en una importante medida, con los recursos públicos recibidos bajo la forma de subsidios y retribuciones concedidos a los miembros del clero que trabajaban en los establecimientos, principalmente monjas de la iglesia católica, culto al que adscribe el Estado argentino.

Durante los gobiernos radicales de 1936 a 1942 se crearon en Córdoba otros cuatro establecimientos estatales de acogida de menores: la Colonia Hogar de Menores Huérfanos y Desamparados Dr. Vélez Sarsfield - cuya piedra fundamental se había colocado en $1919^{\mathbf{1 3}}$-, el internado de la Escuela del Trabajo Presidente Roca, un internado en Villa Belgrano y el Hogar de Menores Madres, establecimientos todos que contaban con una orientación general vinculada al control de los comportamientos y la formación para el trabajo. Al finalizar la década del '30 se había modificado el Código de Procedimiento Penal de la provincia, creándose los juzgados de Instrucción y Menores, magistraturas penales a las que se delegaban las causas que involucraran a menores de edad, con competencia en los "casos en que se deba resolver sobre la persona o derechos de un menor en estado de orfandad, abandono material o peligro moral; o cuando se infrinjan, por los menores o sus padres o tutores, guardadores o terceros, las disposiciones referentes a la instrucción y al trabajo de aquéllos». ${ }^{\mathbf{1 4}}$ Ortiz Bergia comenta que Vélez Mariconde, uno de sus artífices y jurista de renombrada trayectoria local, señalaba que el propósito de estos jueces era realizar una labor preventiva y represiva del problema.

En 1938, por un decreto del gobierno provincial, se crea una Comisión Protectora de la Infancia Abandonada, de carácter honorario, integrada por un magistrado del fuero local y siete «señoras», que tenía por propósito contribuir «desde su órbita a la protección de la niñez y la integración de su tutela pública». ${ }^{15}$

13 «Asilo y Colonia de Menores Varones», diario Los Principios, 25/4/1919.

14 Citado en Ortiz Bergia (2009b).

15 Integraron la comisión José León Schwartz y las «señoras» Lucía Pereyra Duarte de Gallardo, María Cristina Magnasco de Cabral, Lola Álvarez de Vexenat, Blanca Olmos de Tissera, Clara González de Luque, Clara Renella de Monjo, Reyna Loustau Bidaut. Archivo de Gobierno, Serie Gobierno, 1938, T4, Dto. 40.517, 23/6/38. Fue reemplazada siete años después por la Dirección General de Menores, considerándose que «la práctica ha 
Durante aquellos años se había presentado en cuatro oportunidades un proyecto de creación del fuero de menores estructurado por Jorge Eduardo Coll, jurista bonaerense consagrado entre los expertos de la minoridad, con notable influencia en las esferas gubernativas de aquella década: fue presidente del Patronato Nacional de Menores de Buenos Aires en 1933, organizador de la «Primera Conferencia sobre Infancia Abandonada y Delincuente» del país, que se celebrara aquel año, y Ministro de Justicia e Instrucción Pública durante la presidencia de Ortiz (1938-1940). El proyecto en cuestión proponía la creación de un tribunal de menores en la capital provincial, regulando sus competencias, organización y procedimientos; la conformación de una Comisión Honoraria - integrada por el nuevo magistrado y los vocales del Tribunal Superior de Justicia -, a cargo de la administración de los establecimientos públicos y privados de menores de la provincia; y la creación de una Alcaldía para menores, una Colonia Hogar Agrícola Industrial «del sistema denominado «Casas-Hogares»», y una Escuela Profesional de Menores mujeres, establecimientos destinados a la enseñanza de oficios. Ideaba un tribunal a cargo de un juez letrado que contaría con el auxilio de un variado conjunto de colaboradores: un fiscal, un asesor letrado, dos secretarios, un médico especialista en psicología y psiquiatría, «un subsecretario o subsecretaria general de libertad vigilada», cuatro delegados de libertad vigilada (dos varones y dos mujeres), dos oficiales («uno de ellos mujer»), dos escribientes, un auxiliar notificador, un ordenanza. El objeto del proceso debía ser «comprobar el abandono material o moral o el peligro moral del menor, para proceder a su protección». El procedimiento constaba de audiencias y distintos informes tendientes a «conocer especialmente las características de su personalidad: capacidad mental, afectividad, tendencia, hábitos, instrucción y todas aquellas circunstancias de orden moral o del ambiente en que el menor ha vivido». La absolución, la libertad vigilada y

demostrado la poca eficacia, si no el fracaso, de las comisiones «ad-honorem» para dirigir las relaciones del Estado con los menores de cuya custodia se encarga. Ello se debe principalmente a que los componentes de tales comisiones, casi siempre hombres de buena voluntad y elevados sentimientos, no pueden entregarse con la dedicación y el celo necesarios a tan alta función, pues se hallan absorbidos por sus propias ocupaciones, precisamente por carecer de retribución económica». Archivo de Gobierno, Serie Gobierno, 1945, T96, Dto. 13.520, 5/3/45, ratificado por Decreto 12.347/45 del Gobierno de la Nación. 
la internación serían los destinos posibles para quienes fueran objeto de aquella intervención judicial. ${ }^{\mathbf{1 6}}$

Algunos indicios habilitan a pensar que las ideas respecto de la intervención estatal sobre menores eran compartidas en el ámbito nacional y provincial. Que el proyecto impulsado en cuatro oportunidades por legisladores radicales y socialistas haya sido elaborado por Jorge Eduardo Coll, y que promoviera los mismos establecimientos y la misma organización judicial y ejecutiva se implementaran en Buenos Aires y a partir de ella en Mendoza y Santa Fe, son elementos que abonan aquella afirmación. No obstante, la divergencia de experiencias entre Córdoba y el centro hegemónico del país podría vincularse tanto a razones de orden económico como a aquella postura disidente que caracterizara para Philp la relación entre el gobierno local y el nacional. Sostiene la autora que los gobiernos provinciales radicales entre 1936 y 1943 eran un foco de resistencia al gobierno central; con proyección nacional, bregaban por la restauración de la autonomía provincial y proyectaban un nuevo modelo de Estado de carácter intervencionista. ${ }^{17}$ Durante la década del ' 40 el gobierno provincial experimentó una sucesión de intervenciones federales por cambios de gobierno a nivel nacional (golpe de Estado de 1943) y conflictos partidarios a nivel provincial. ${ }^{18} \mathrm{~A}$ ellas debemos en materia de políticas de minoridad la creación por decreto de la Dirección General de Menores en 1945 - dependencia del poder ejecutivo que gobernaba los establecimientos de menores en la provincia y decidía sobre la internación y el egreso de los niños -, las becas de estudio para «niños varones huérfanos o abandonados» ${ }^{19}$ en 1947 , y la reorganización del Resguardo de Menores de la Cárcel de Encausados en 1949. ${ }^{\mathbf{2 0}}$

En la provincia de Buenos Aires entonces, allí donde los especialistas en minoridad arribaron a posiciones políticas de relevancia y delinearon una política que sería un norte para otras provincias, la estructura institucional de la minoridad estaba montada al iniciarse la década de 1950. Recapitulando, se cuenta con un fuero especial, establecimientos estatales de acogida y

16 Tribunales de Menores (1942).

17 Philp (1998) 61-64, 76-83.

18 Ibídem 142.

19 Archivo de Gobierno, Serie Gobierno, 1947, T8, Dto. 242, 25/1/47. Hacia 1953 se observa entre las resoluciones de la Dirección General de Menores una notable concesión de estas becas.

20 Archivo de Gobierno, 1949, T75, Dto. 6.118, 19/12/49.

234 Agostina Gentili 
reclusión de menores junto a los de origen privado que nunca dejarán de existir, y un organismo estatal encargado de coordinar el accionar de esos establecimientos. En Córdoba, durante aquellos años se habría privilegiado la construcción de instituciones de internación, una solución provisoria en sede judicial al asignarse los asuntos de menores a magistrados del fuero penal, que convivía desde la década de 1920 con las defensorías de menores, y una solución de más largo alcance en sede ejecutiva, con la creación de la Dirección General de Menores como dependencia encargada de centralizar la acción de los establecimientos públicos y privados de menores, y administrar el ingreso y el egreso de niños y jóvenes en esos espacios. Se intenta señalar entonces que las diferencias que hacia 1950 presentaba Córdoba respecto de Buenos Aires en la organización institucional de la minoridad parecen responder a razones de orden económico y político, más que a criterios divergentes de organización. Abona esa hipótesis la creación del fuero de menores a fines de los años '50, por un decreto del poder ejecutivo provincial, nuevamente intervenido desde el gobierno nacional.

Considerando que en la Justicia provincial las causas de menores se llevaban de una forma totalmente contraria a los fines de la ley nacional que instaurara en 1954 un régimen penal especial para menores de edad («la preservación en la mayor medida posible de la personalidad moral de los menores, y el resguardo de la seguridad y de la tranquilidad social comprometidas por la acción de aquéllos»), el gobierno provincial del interventor de facto constituye en 1956 una comisión que elabora el proyecto de creación del fuero de menores. ${ }^{21} \mathrm{La}$ integraron, entre otros, dos juristas con trayectoria en el fuero penal de la provincia, Ricardo Núñez y Ernesto Gavier, que en esos años habían sido designados en el Tribunal Superior de Justicia, y el encargado de la Dirección General de Menores, Francisco Torres.

El ejecutivo provincial se mostraba preocupado por el aumento de los delitos atribuidos a menores, pero aun más porque a sus ojos en las estadísticas los niños aparecerían como «instrumentos» de los mayores, que encontraban provechoso «utilizarlos [...] sin temor a una reacción efectiva de parte del Estado». Se entendía que ello tenía lugar por la falta de una "centralización jurisdiccional preventiva, correctiva y punitiva en materia de menores», en la imposibilidad de aprovechar adecuadamente los medios prácticos y las instituciones con que contaba la provincia, y por la falta de un estable-

21 Archivo de Gobierno, Serie Gobierno, 1956, T23, Dto. 5.700, 14/11/56. 
cimiento represivo especial para menores. Se agregaba a eso el problema de niños víctimas de delitos, abandonados material o moralmente o con «desórdenes de conducta». Con base en «las experiencias hechas por otros estados argentinos», se entendía que el «remedio» a esa situación era la organización de «un organismo jurisdiccional y tutelar que por sus facultades y por su unidad de acción pueda no sólo desenvolverse con prontitud y eficacia sino también contar con el auxilio irreemplazable de la especialización técnica en la materia». Se promovía entonces la creación de una magistratura de menores complementada técnica y ejecutivamente por un «cuerpo de patronato especializado», para tutelar no sólo a los menores sino también a la sociedad «frente a los daños y los peligros provenientes de la inconducta o del desamparo de aquéllos». ${ }^{22}$

Con esa orientación se creó en Córdoba el primer juzgado de menores. Un magistrado y cuatro funcionarios, uno por cada rama de este fuero que acogía distintas materias legales, comenzaron a atender en sede judicial las cuestiones que involucraban a menores de edad. Las secretarías Sanción y Corrección eran el brazo penal del fuero; la primera aplicaba el Código Penal a personas de entre dieciséis y dieciocho años, y la segunda aplicaba un régimen penal especial a menores de dieciséis. La Secretaría Civil tramitaba cuestiones relativas a alimentos, adopciones, tutelas, venias, entre otras, y la Secretaría Prevención las situaciones que involucraban a víctimas de delitos, abandono o a quienes estaban en peligro material o moral, expresión recurrente del argot de la minoridad. Un fiscal y un asesor ad hoc conformaban el ministerio público del fuero.

La Dirección General de Menores pasó desde entonces a ser el órgano de colaboración, ejecución y asistencia de la labor judicial, con una policía ad $h o c$, la policía juvenil. De aquélla seguían dependiendo los establecimientos, y aún era su misión la «asistencia integral del menor, desde su estudio ambiental en la familia, en la sociedad, su ingreso, corrección de desviaciones de conducta y reeducación orientada al aprendizaje de oficios manuales, fabriles y rurales y a lograr la plena salud física y moral». ${ }^{23}$

La nueva magistratura tendrá competencia en la ciudad de Córdoba y localidades cercanas; los jueces de instrucción continuarán ocupándose de las causas de menores en otras localidades, por limitaciones económicas. A esas

22 Ibídem.

23 Creación del Patronato y la Magistratura de Menores (1957).

$236 \quad$ Agostina Gentili 
mismas limitaciones parece responder la conformación del fuero, diferente a la propuesta en iniciativas locales anteriores y a la que tendría en otras provincias: el juzgado cordobés no contará con un cuerpo de delegados, médicos y asistentes sociales propios, tareas a cargo de la Dirección General de Menores.

El decreto de creación del fuero se publicó en los Anales de legislación argentina con el título «Aplicación del régimen penal y correccional de los menores de 18 años». ${ }^{24}$ El carácter penal del problema de la minoridad había sido una constante a lo largo de todo el período estudiado, y se volvía ahora el principal fundamento de la creación de un fuero. La minoridad en el ámbito judicial así lo había perfilado, con la existencia de un «Patronato de menores, presos y liberados» en su organización, y la asignación de las causas de menores a jueces de instrucción. La organización de las secretarías del nuevo juzgado tenía también esta impronta: tres de las cuatro llevaron denominaciones propias del lenguaje penal: prevención, sanción y corrección. Su ubicación cercana al fuero penal en el edificio de Tribunales y la asignación de los mismos horarios de trabajo para facilitar la «comunicación frecuentemente necesaria - entre los tribunales de una misma materia», fueron también elementos de aquella idiosincrasia. ${ }^{25}$ De allí la impronta penal de un fuero híbrido, que con aquellas orientaciones atendía distintas materias legales.

\section{Escenario de convalidaciones, negociación y servicios}

He reconstruido hasta aquí la estructura institucional de la minoridad, organizada, como se señalara, en torno a una magistratura de menores que laboraba junto a un organismo administrativo. Avanzaré ahora hacia el análisis de las actuaciones de este nuevo fuero, a partir de un acercamiento a un grupo particular de expedientes: las guardas de niños, esto es, los procesos que tenían por finalidad nombrar a las personas que estarían a cargo de esos niños, convivirían con ellos en sus casas, en las que debían, según rezaban los mandamientos judiciales, tratarlos «como hijos» 0 "personas de familia». Es decir, se propone observar el accionar del fuero allí donde lo que se define es

24 Aplicación del Régimen Penal y Correccional de los Menores de 18 Años (1957).

25 Archivo General de los Tribunales de Córdoba, Libro de Acuerdos del Tribunal Superior de Justicia, $\mathrm{N}^{\circ}$ 21, 1962, Acuerdo $\mathrm{N}^{\circ}$ 9, 15/2/62. 
la ubicación de los niños en el ámbito privado, la casa de sus guardadores, principal escenario de las relaciones familiares. Al estudio de esos procesos se dedican ya dos años de trabajo, en el marco de una investigación doctoral en historia sobre las entregas de niños tramitadas entre 1957 y 1974 en los juzgados de menores cordobeses. Como sostiene Adriana Vianna - antropóloga brasilera que estudió expedientes de guarda tramitados en Río de Janeiro entre 1980 y 1990 -, una guarda se produce a través del establecimiento de «relaciones complementarias», aunque asimétricas, entre la administración y las casas en las que los niños se encuentran o a las que serán destinados. En esa relación, la transferencia de las responsabilidades sobre menores depende del diagnóstico administrativo de los contextos, que incluye la identificación de acciones moralmente condenables, y de la eficacia de quienes gestionan cotidianamente situaciones que los involucran. ${ }^{26}$

Los documentos en estudio forman parte del Centro de Documentación Histórica del Poder Judicial y se conservan en el Archivo General de los Tribunales de la provincia. Al fondo lo constituyen cerca de 4.000 expedientes tramitados por el fuero de menores en aquellos años, que por la ley de expurgo vigente desde 1977 deberían haberse eliminado una vez transcurridos diez años de su envío al archivo. Razones ocasionales atentaron contra aquel destino, haciendo posible que fueran reunidos años después y sean hoy de acceso público. No se cuenta entonces con una serie completa de documentos, sino con los restos de que lo alguna vez se envió al archivo. Entre ellos, cerca de 1.700 corresponden a la Secretaría Prevención, donde se tramitaban las guardas. Para identificarlas he recorrido más de veinte cajas que contienen 1.112 expedientes (el 65\% de los existentes de la secretaría), entre los que hallé 472 expedientes de guarda. Dado que la confección de las carátulas no necesariamente suponía la consignación del asunto judicial del que trataba el expediente, fue necesario leer cada uno para identificar qué contenían, tarea que no sólo habla del oficio del historiador, sino también de las prácticas del fuero. Agrupar las acciones del proceso bajo la genérica denominación de "prevención» es una recurrencia de 297 expedientes (el 63\% de los identificados). Para María Lugones - que armó el fondo en estudio $^{27}$ y dedicó su tesis de maestría a reflexionar sobre el sentido tutelar del patronato estatal, indagando las formas de gestión de la minoridad en

26 Vianna (2010).

27 Lugones/RufER (2004).

238 Agostina Gentili 
Córdoba a partir del análisis etnográfico de expedientes prevencionales -, al caratular los expedientes los juzgados no sólo convierten la heterogeneidad de la vida social en casos administrativos, sino que también ocultan, enmascaran esa diversidad. ${ }^{\mathbf{2 8}}$

El corpus de guardas fue organizado a partir de dos grandes grupos de personas que solicitaron ser guardadoras de los niños: familiares y terceros personas ajenas al parentesco biológico - (199 y 280 procesos respectivamente, cifras que no reflejan la cantidad de expedientes, por cuanto en algunos se concedieron ambos tipos de guarda). En su mayoría estos procesos muestran trámites motorizados por particulares. Las solicitudes más comunes, bajo la forma de una declaración, permiten reconstruir la escena de un hombre, una mujer o ambos, que solicitan a un funcionario judicial que legitime la tenencia de un niño que ya está a su cargo. No se encuentran entregas impulsadas por las propias autoridades judiciales, lo que quizá sea sólo el efecto provocado por documentos que ellas mismas producen valiéndose de fórmulas escriturales. En sólo 84 expedientes es de los llamados institutos u hogares de menores, hospitales infantiles y maternidades de donde derivaban los niños y jóvenes solicitados en guarda; se reitera en ellos la iniciación del trámite judicial por parte de los particulares interesados, que presentaban notas e informes producidos por el personal de aquellas instituciones.

Por lo general, al pedido de guarda le seguía la concesión de una guarda provisoria hasta tanto concluyera el trámite judicial. La legislación del fuero prescribía para todo proceso prevencional tres requisitos burocráticos. ${ }^{29} \mathrm{La}$ realización de «informes técnicos», que en estos casos consistían en encuestas ambientales y familiares realizadas por asistentes sociales que visitaban la casa de quienes deseaban guardar al niño, o bien personal policial o jueces de paz cuando los interesados en la guarda vivían fuera del ejido urbano. La encuesta ambiental y familiar era un instrumento al que se supeditaba la resolución judicial, principal herramienta de intervención del fuero que permitía a magistrados y funcionarios ver aquello que no veían durante el proceso: la casa donde el niño habitaba. Estas piezas burocráticas presentaban, bajo un mismo criterio de confección, los datos personales de quienes convivían en la casa, las características de la vivienda - desde los materiales, el número y tipo de habitaciones, su ventilación y aseo, hasta los servicios - y una reseña del

28 Lugones (2004) 29-36.

29 Artículo $17^{\circ}$ Decreto ley 6.986/57 y artículo $16^{\circ}$ Estatuto de la Minoridad, Ley 4.873/66. 
caso en la que la asistente social relataba su entrevista al mayor que la recibía durante la visita, contando sobre los medios y modos de vida de los habitantes de la casa y recabando opiniones personales de sus vecinos. Los informes concluían con la opinión personal de la asistente social respecto de la guarda pedida, y pasaban por sucesivas remisiones burocráticas antes de llegar al juzgado y de allí al expediente. No eran las asistentes sociales quienes los firmaban, sino sus jefas en el Departamento de Servicio Social de la Dirección General de Menores, quienes los remitían a la llamada Secretaría Técnica de la repartición, una de sus máximas autoridades, que tenía entre otras funciones la relación y la comunicación diaria con el juzgado de menores. El camino inverso se recorría cuando se pedía una encuesta a la Dirección General, que se dirigía al Secretario Técnico, que remitía el pedido a la jefa de departamento, y ésta lo asignaba a alguna de sus empleadas a cargo. Los tiempos transcurridos entre el pedido y la incorporación del informe variaban mucho, semanas o meses, constatándose a veces la ausencia en el expediente de aquella pieza burocrática.

La intervención de un asesor de menores en defensa de los derechos del niño era otro de los mandatos legales. Al recorrer los expedientes, se aprecia que cada asesor atendía las causas de un juzgado en particular y tenía sus propios estilos de participación. Por lo general, los expedientes se les remitían una vez que las guardas se solicitaban, respondiendo que esperaban la realización de la encuesta para emitir su opinión, y solicitando, a veces, que se incorporaran documentos que acreditaran identidades y filiaciones enunciadas o silenciadas en las declaraciones o los pedidos de guarda. Las partidas de nacimiento de los niños no siempre formaban parte de estos trámites judiciales. Si bien no eran infalibles, ya que se identifican casos de falsificaciones, eran los principales documentos para conocer quién era el niño y quiénes sus padres, durante un trámite que tenía por objeto incorporarlo a un nuevo espacio privado de convivencia. En la mayoría de los procesos la identidad y la filiación de los niños surgían de lo expresado por los interesados en su guarda o por quienes se presentaban como sus madres. Esta preocupación por acreditar la identidad de los niños o jóvenes respondía a la necesidad de justificar la intervención, esto es, incorporar al proceso un documento que demostrara que era un menor de edad quien había motivado la intervención judicial.

La legislación prescribía también la realización de una audiencia de la que participaran los interesados en la guarda, la asistente social, el asesor de 
menores, el juez y el secretario: la «audiencia de ritual», según es referida en los expedientes. Solía ser la oportunidad en que el magistrado «toma[ba] conocimiento personal del menor»-como refieren algunas de estas piezas burocráticas-, a quien luego se hacía retirar de la sala de audiencia para dar paso al acto procesal en el que, por lo general, cada participante se remitía a lo que ya había expresado con anterioridad: el guardador a su pedido, la asistente social a su informe (que, a decir verdad, no sabemos si lo había producido ella o no, ya que tampoco en esta oportunidad era identificada, y se encuentran casos en que se remitían a informes producidos por jueces de paz) y el asesor, que por lo general no tenía «nada que objetar». Cerrado el acto, las actuaciones estaban en condiciones de arribar a la resolución judicial, un auto interlocutorio que confirmaría la guarda concedida provisoriamente al iniciarse el proceso, concesión que por derivar de una resolución de aquella naturaleza, era revocable.

No es intención de este trabajo ponderar cuánto se acercaban o alejaban los procesos a aquel procedimiento prescripto. La lectura de los expedientes permite observar que aquel recorrido de acciones gravitaba en la concesión de una guarda sin que la ausencia de alguno de sus pasos modificara el destino de los niños involucrados. Como se observa, el procedimiento prescripto y ensayado no se alejaba de aquél que, como se mencionara, fuera practicado desde fines de los años '30 por el fuero bonaerense, refrendado en 1954 por el régimen penal especial para menores de edad. El fuero actuaba a partir de la incorporación de informes, en este caso ambientales y familiares - en otros psicológicos -, que solicitaba a su órgano de colaboración y asistencia, la Dirección General de Menores, ya que no contaba aún con equipos técnicos propios.

Tres sentidos y usos posibles de este fuero sobresalen en las guardas identificadas: la convalidación, la negociación y los servicios. Como se adelantara, en una gran cantidad de procesos el destino del niño junto a sus guardadores estaba definido de antemano, pidiéndose a la autoridad judicial que convalidara una situación de guarda existente. El ingreso a la escuela primaria, con la consiguiente presentación de documentos que identificaran a un mayor como responsable del alumno, solía ser el momento en que esa guarda de hecho necesitaba una legitimación judicial. La aparición de algún familiar del niño luego de muchos años y el temor de los guardadores a que deseara llevárselo son otros de los desencadenantes de aquellos pedidos; en estos casos, cabe señalar que las decisiones judiciales se inclinaban a mante- 
ner el statu quo, es decir, que el niño permaneciera junto a quienes ya lo venían criando, obviándose la citación de sus madres biológicas durante el proceso.

El fuero se volvía escenario de negociación en aquellas situaciones en que se acudía al juzgado ante los conflictos de parejas o entre padres e hijos. Ello se observa en buena parte de los procesos iniciados por las madres o los padres de los niños al haberse separado la pareja. En el conjunto revisado, fueron 66 las madres que solicitaron guardas de sus hijos. Salvo dos, que podían incluirlos en los servicios sociales porque trabajaban en relación de dependencia o sus maridos por cuenta propia, el resto no estaba junto al padre de los niños. 36 dijeron haberse separado y ser ésa la razón por la que pedían la guarda. Eran, en su mayoría, mujeres casadas; algunas volvieron con sus parejas, pidiendo el archivo de las actuaciones por haber «reiniciado la vida en común». Hacer del escenario judicial un lugar de negociación era también parte de la estrategia de algunos jóvenes que no deseaban seguir viviendo con sus padres y, habiendo encontrado quién se hiciera cargo de ellos, acudían al juzgado solicitando se les nombrara guardador.

El trabajo es otro de los posibles destinos de los jóvenes en guarda. En 34 expedientes se conceden guardas a "patrones», tal como son referidos en los informes. En algunos casos esta ubicación se daba por arreglos previos entre padres y patrones, pero en otros era recurrente la ubicación de las jóvenes internadas en instituciones estatales - particularmente el Asilo del Buen Pastor - como empleadas domésticas o para «ayudar en las tareas de la casa» de sus guardadores. Antigua práctica colonial, la «colocación» de los niños en lugares donde «se les dé algún oficio o profesión susceptible de proporcionar los medios de vivir» era una de las atribuciones de los defensores de menores. ${ }^{30}$ La práctica se prohibió en 1966, haciéndose referencia a «la necesidad de terminar con los viejos resabios de las Defensorías de antaño que con carácter paternalista protegían al menor entregándolo para el servicio doméstico», según argumentara el gobernador Justo Páez Molina al presentar el proyecto que dio lugar a un nuevo régimen provincial de la minoridad, aclarando: «no se descarta la posibilidad de que las condiciones intelectuales de un menor sólo le permitan dicho tipo de trabajo, pero a ello debe llegarse cuando están agotadas todas las instancias pedagógicas.» ${ }^{31}$ La prohibición

30 Artículo $98^{\circ}$ Ley Orgánica del Poder Judicial de Córdoba № 3.364.

31 Estatuto de la Minoridad (1965c) 2.582.

242 Agostina Gentili 
abonaría una de las tesis de Lugones: que entre funcionarios judiciales y monjas del Asilo del Buen Pastor existía un «circuito de provisión de servicio doméstico» en el que la administración judicial estaría operando como intermediaria entre patrones y jóvenes. ${ }^{32}$ Con posterioridad a su prohibición la práctica continúa vigente. Referencias elípticas hacen notar su presencia, como la de aquellos guardadores de jovencitas que se comprometen a darles «trato de familia», cuando antes no había un compromiso explícito en tal sentido.

\section{Últimas palabras}

Preocupaciones penales e idiosincrasias de aquel mismo ámbito gravitaban sobre este fuero especial que en torno a un sujeto social particular acogía distintas materias legales. La reconstrucción histórica de la estructura institucional de la minoridad en Córdoba intentó demostrar que no fueron proyectos divergentes respecto de qué hacer y cómo organizar la intervención estatal sobre menores, sino razones de índole económica y política, las que definieron una trayectoria desfasada y una diagramación particular respecto del centro hegemónico del país. En la trayectoria nacional y provincial de la minoridad, el crecimiento institucional orientado hacia una mayor especialización en la intervención y el seguimiento en este proceso de los lineamientos de los países centrales de Occidente, fueron rasgos asociados a los componentes «modernos» de la organización del Estado. Y así como existió en el país una propensión marcada a nortear los diseños institucionales en función de los de las naciones «modernas», los gobernantes provinciales asumieron esa misma orientación respecto de Buenos Aires. El accionar mismo de los juzgados de menores de Córdoba, al que accedimos a partir del análisis de expedientes de guarda, da cuenta de la pervivencia de una matriz de intervención compartida con el fuero bonaerense, centrada en la evaluación de la conducta del menor y de su ambiente. Ofreciendo éste un espacio de contención, vigilancia y educación, la autoridad judicial no interfería en las decisiones que los particulares definían de antemano. 


\section{Bibliografía}

Aplicación del Régimen Penal y Correccional de los Menores de 18 Años, Decreto ley $N^{\circ} 6.989$ del 26/3/57, Anales de Legislación Argentina, Buenos Aires: La Ley (1957), 1542-1545

Creación del patronato y la magistratura de menores, Decreto ley $\mathrm{N}^{\circ} 6.989$ del 26/3/ 57, Suplemento Comercio y Justicia (1957), Tomo VII, 158-160

Estatuto de la Minoridad, Diario de sesiones de la Cámara de Diputados de Córdoba (1965a), Tomo I, 313-323

Estatuto de la Minoridad, Diario de sesiones de la Cámara de Senadores de Córdoba (1965b), Tomo II, 1.784-1.807

Estatuto de la Minoridad, Diario de sesiones de la Cámara de Diputados de Córdoba (1965c), Tomo III, 2.569-2.628

Lugones, María Gabriela (2004), Uma «visita» aos «expedientes»: os processos judiciais referentes aos menores. Córdoba, Argentina, na mitade do século XX, disertación de maestría, Programa de Postgraduación en Antropología Social, Museo Nacional, Universidad de Río de Janeiro

Lugones, María Gabriela, Mario Rufer (2004), Fuentes en extinción: estudio cualitativo de procesos judiciales de los Tribunales de Menores de Córdoba, en: Miradas alternativas 2, 37-44

Modificaciones al Régimen de los menores y de la familia, Código Civil de la República Argentina (con las notas de Vélez Sársfield) y Leyes Complementarias, Buenos Aires: Lajouane Editores 1956, 896-906

Ortiz Bergia, María José (2009a), De caridades y derechos. La construcción de políticas sociales en el interior argentino. Córdoba (1930-1943), Córdoba: Centro de Estudios Históricos «Profesor Carlos Segretti»

Ortiz Bergia, María José (2009b), Los menores abandonados y la tutela estatal en Córdoba, cambios y continuidades en un período de transición (1930-1943), en: Síntesis 3, en prensa

Philp, Marta (1998), En nombre de Córdoba. Sabattinistas y peronistas: estrategias políticas de construcción del Estado, Córdoba: Ferreyra Editor

Stagno, Leandro (2010), Una infancia aparte. La minoridad en la provincia de Buenos Aires (1930-1943), Buenos Aires: Libros Libres

Tribunales de Menores, Diario de sesiones de la Cámara de Senadores de Córdoba $1942,620-628$

Vianna, Adriana(2010), Derechos, moralidades y desigualdades. Consideraciones acerca de procesos de guarda de niños, en: Villalta, Carla (comp.), Infancia, justicia y derechos humanos, Buenos Aires: Universidad Nacional de Quilmes, $21-72$

Zapiola, Carolina (2010), La Ley de Patronato de Menores de 1919. ¿ Una bisagra histórica?, en: Lioneti, Lucía, Daniel Míguez (comp.), Las infancias en la historia argentina. Intersecciones entre prácticas, discursos e instituciones (1890-1960), Rosario: Prohistoria, 117-132 


\title{
Un codigo para el desierto argentino
}

\author{
La discusión en torno a la propiedad del ganado en el discurso \\ académico de la primera mitad del siglo $\mathrm{XX}^{1}$
}

La cuestión de la modernización, y en especial la de los países latinoamericanos, ha atraído la mirada de numerosos investigadores, provenientes de los más variados ámbitos disciplinares. Es una problemática que sigue generando múltiples inquietudes que van desde la conceptualización misma del fenómeno hasta la verificación de sus impactos y efectos concretos en las distintas culturas locales. $^{2}$

La construcción de los distintos estados nacionales durante el siglo XIX, no solo acompañó sino que también propició estas transformaciones que afectaban por igual los campos políticos, económicos y culturales. La búsqueda del progreso era alimentada por el magnetismo que tanto Europa como Estados Unidos, considerados verdaderos «focos de cultura» producían sobre las elites latinoamericanas. Resultaba, también, de complejos procesos de intercambio que se caracterizaban por la pluralidad de elementos que intervenían en los mismos: agentes culturales y científicos, redes personales y profesionales, viaje de estudios, revistas científicas y negocios editoriales, intercambios epistolares, visitas académicas, etc. ${ }^{3}$

Un punto de partida significativo en nuestro estudio lo constituye el movimiento codificador que en nuestro país se materializó en el último

1 El presente trabajo fue presentado en una versión preliminar en el marco del Seminario «Derecho privado y modernización en América Latina y Europa en la primera mitad del siglo XX», organizado por el Max Planck Institut für europäische Rechtsgeschichte y el Instituto de Investigaciones de Historia del Derecho y celebrado en la sede de éste último los días 4 a 6 de julio de 2012. Agradezco los comentarios que me hicieron entonces, especialmente los de Eduardo Zimmermann, Thomas Duve y Ezequiel Abásolo.

2 Por nombrar algunos análisis: Schwarz (1977); y una crítica a esta tesis Palti (2007) 259-308; Palti (2008); Dotti (1990); García Canclini (2010).

3 Botana, Gallo (2007); García Sebastiani (2008); Altamirano (2008); Zimmermann (2008). 
cuarto del siglo XIX con la sanción de los distintos códigos. Como lo ha señalado Tau Anzoátegui, la idea de codificación iba acompañada de una profunda crítica del ordenamiento vigente, considerado no solo vetusto sino inadecuado como expresión de los principios políticos y jurídicos consagrados por el movimiento emancipador. ${ }^{4}$ El código aparecía como una instancia válida para la reforma de la legislación y de las prácticas judiciales proyectadas y por lo tanto un paso fundamental para la modernización jurídica que permitirían a nuestro país, incluirse en el concierto de las naciones "civilizadas». 5

La Constitución de 1853 se decidió por el sistema de códigos, ${ }^{6}$ que al atribuir su sanción al Congreso Nacional, acentuaba el centralismo político, en detrimento del sistema federal que ella misma organizaba. ${ }^{7}$ Esta solución encontraba asidero entre un grupo importante de nuestros intelectuales, para los cuales el progreso político implicaba superar los poderes locales encerrados en las provincias. Incluso para Alberdi, contrario a la codificación «la legislación civil y comercial argentina debe ser uniforme como ha sido hasta aquí. No sería racional que tuviésemos tantos códigos de comercio,

4 Tau Anzoátegui (2008) 163 ss.

5 Así sostenía Velez Sarsfield en defensa de su proyecto de código civil frente a las críticas de Alberdi: «Yo me proponía que en mi código apareciera el derecho científico, como lo llaman los alemanes al derecho que la ciencia establece, las doctrinas de los más acreditados jurisconsultos; que en él se viese, si era posible, el estado actual de la ciencia, si yo alcanzase a tanto; y por esto justifico las resoluciones del código con los escritores más conocidos de todas las naciones», Velez Sarsfield (1920) 242.

6 Pudo no haberlo hecho, siguiendo en este punto la recomendación de Alberdi que, crítico acérrimo de los códigos, proponía la renovación del ordenamiento civil mediante leyes parciales: «Las exigencias económicas e industriales de nuestra época y de la América del Sud deben servir de base de criterio para la reforma de nuestra legislación interior, como servirán para la concepción de nuestro derecho constitucional ... Para obrar estos cambios tan exigidos por nuestro adelantamiento, no es menester pensar en códigos completos. Las reformas parciales y prontas son las más convenientes. Es la manera de legislar de los pueblos libres. La manía de los códigos viene de la vanidad de los emperadores». Alberdi (1915) 106, 109-110. Ver también Tau Anzoátegui (2008) 361-362.

7 El artículo 64, inc. 11 del texto constitucional de 1853 establecía que correspondía al Congreso de la Nación «Dictar los códigos civil, comercial, penal y de minería, y especialmente leyes generales para toda la Confederación sobre ciudadanía y naturalización, sobre bancarrotas, sobre falsificación de moneda corriente y documentos públicos del Estado, y las que requiera el establecimiento del juicio por jurados», mientras que el artículo 105 prohibía a las provincias «dictar los códigos Civil Comercial, Penal y de Minería, después que el Congreso los haya sancionado». 
tantas legislaciones civiles, tantos sistemas hipotecarios, como provincias. La uniformidad de la legislación, en esos ramos, no daña en lo mínimo a las atribuciones de soberanía local, y favorece altamente el desarrollo de nuestra nacionalidad argentina». ${ }^{\mathbf{8}}$ También las palabras de Velez resultan sumamente elocuentes para nuestro análisis:

«Entre nosotros, son Estados todas las desmembraciones que sucedieron durante la anarquía de treinta años, tengan o no medios propios de existencia; puedan o no darse una legislación digna de la época en que vivimos y en armonía con la legislación política ... Con códigos generales salvamos los primeros derechos de los hombres, aunque por tiempo ilimitado desaparezca en mucha parte la soberanía provincial. Así también continuamos el orden bajo el cual nacieron y se formaron esos pueblos. Una legislación civil uniforme en todo el territorio los había regido y rige hasta ahora, legislación que ha creado costumbres también uniformes y por la cual los derechos relativos son perfectamente iguales. Pero hemos dicho que un código nacional, aunque tenga ventajas incontestables, destruye en mucha parte la soberanía de las provincias. Esto es sólo un mal temporal que otro día puede cesar sin que se altere la Constitución de la Nación. Cuando las provincias se hallan en estado de darse sus leyes civiles, el Congreso puede retirar la sanción que hubiese dado el código civil, y quedarán los pueblos con capacidad legal para reformarlo o darse otras leyes civiles; pero siempre tendremos un precedente muy feliz en el orden social: el haber tenido las provincias una misma legislación civil». ${ }^{9}$

A pesar de las intenciones de reformas esgrimidas, la sanción del código civil no abrogó completamente el orden jurídico anterior. ${ }^{\mathbf{1 0}}$ Pero la abstracción que él imponía no hacía más que legitimar y naturalizar algunos de los postulados ideológicos del derecho liberal (el carácter absoluto de la propiedad privada, la autonomía de la voluntad, la libertad de contratar, etc.), presentándose a sí mismo, como un programa racional y científico, instrumentado desde el estado nacional, que implementaría paulatinamente un nuevo orden jurídico en reemplazo del tradicional. ${ }^{\mathbf{1 1}}$ Lentamente, se iría desarrollando, en algunos ámbitos, lo que se ha denominado la «cultura del código", concibiéndose al mismo como expresión y fundamento de nuestro orden civil. ${ }^{\mathbf{1 2}}$

8 Alberdi (1915) 110.

9 El subrayado es nuestro. Velez Sarsfield (1920) 240-242.

10 Incluso en algunas de sus partes lo reenviaba a él, como en el caso de la celebración del matrimonio que Vélez remitía al mismo derecho canónico.

11 Caroni (2012) 49 ss.

12 Tau Anzoátegui (1998). Este autor acuñó para nuestro medio el tópico «cultura del código», como uno de los rasgos dominantes del derecho argentino en el siglo XX. Bajo esta 
De ahí que nuestra pregunta en la presente investigación vaya dirigida a dilucidar la idea de la existencia de un derecho moderno, el valor del código civil frente al resto del ordenamiento iusprivatístico, la posibilidad de regular el derecho de propiedad fuera del código civil, el valor de los ordenamientos locales, y la operatividad de ciertas tradiciones jurídicas contenidas en los códigos rurales. Para ello nos centraremos en las controversias en torno a la propiedad del ganado en el discurso académico de la primera mitad del siglo $\mathrm{XX}$, entendiendo que la misma nos ofrece un observatorio privilegiado para el análisis de estas cuestiones.

\section{Del centro de la economía a la periferia del derecho}

En los estudios historiográficos existe el consenso que el avance de la modernización de la Argentina se encuentra íntimamente relacionado con el proceso de organización nacional desencadenado con la constitución de 1853 y consolidado a partir de $1880 .{ }^{13}$ Esta modernización tenía como trasfondo el afianzamiento de un modelo agroexportador, que se venía estructurando desde fines del siglo XVIII, y que encontraba en la construcción de un poder nacional la condición necesaria para su desenvolvimiento. ${ }^{\mathbf{1 4}}$

En un sentido, modernizar implicaba la implantación de un orden que «excluyese todos aquellos elementos que podían obstruir el progreso, el avance de la civilización»; estas rémoras, «eran una amenazadora realidad presente, vestigios de una sociedad cuyos parámetros se pretendía transformar». ${ }^{15}$ Entre ellas, para algunas élites, se encontraba la tradicional estructura del orden político, considerada heredera del orden colonial, que se fundamentaba principalmente en relevancia del poder local.

Algunas voces se levantaban contra este esquema de poder, planteando la necesidad de reformular el sistema federal instaurado en la constitución de 1853 y consolidado en la reforma de $1860 .{ }^{\mathbf{1 6}} \mathrm{Si}$ para algunos el federalismo

denominación, cabe entender la concepción que hizo del código el objeto preferente, cuando no exclusivo, de estudio y que impuso un modelo de razonar ajustado a estrechas pautas. Ver también: Leiva (1988) y Tau Anzoátegui (1982); Zimmermann (1999); Polotto (2012).

13 Ver el análisis de Oszlak (2011).

14 OszlaK (2011) 48 ss.

15 Oszlak (2011) 59.

16 Botana, Gallo (2007) 81 ss. Zimmermann (1998) 145 ss. 
argentino implicaba una evolución de nuestras formas políticas que tenía hondas raíces en los «precedentes gubernativos y las inclinaciones políticas heredadas de España», ${ }^{17}$ para otros implicaba la concreción burda de un «pacto de conservación entre capitanejos». ${ }^{\mathbf{1 8}}$ En síntesis, para aquellas elites comprometidas con la idea de progreso, modernizar implicaba si no integrar, por lo menos neutralizar el peso de estos poderes locales.

La economía argentina encontraba su estímulo en el empuje europeo, que ayudó a transformarla en una de las economías de exportación más exitosas de América Latina, lo que hasta el siglo XVIII había sido un área marginal del imperio español. ${ }^{19}$ El epicentro de este crecimiento, se encontraba en la provincia de Buenos Aires que concentraba más de la mitad de la producción exportable argentina, diseminándose el resto en las demás provincias pampeanas y del interior del país. ${ }^{20}$

En un primer momento, la aceleración del desarrollo capitalista en la pampa afectó de un modo superficial a la mayoría de los empresarios con intereses rurales, que continuaron administrando sus negocios de modo consuetudinario, ${ }^{\mathbf{2 1}}$ acompañados de un orden político también tradicional, considerado heredero del pasado indiano, que se apoyaba, fundamentalmente, en la relevancia del poder local. ${ }^{22}$ Todavía en el último cuarto del siglo XIX, la provincia constituía el marco de referencia de las relaciones sociales, y la soberanía provincial, la condición de legitimidad de los actos del gobierno nacional. $^{23}$

17 Matienzo (1917) 60-61.

18 Rivarola (1908) 22. Para este autor el progreso se encontraba íntimamente unido a la consolidación de una estructura unitaria que fortaleciese el poder nacional: «Sin embargo, puede ya decirse que cincuenta y cinco años de experiencia bastan para prueba, por lo menos, de que la Constitución federal de ocasión, cumplió su oficio transitorio, y es hora de acompañar con el pensamiento y con las condensaciones de opinión que constituyen un partido, la evolución unitaria, que implica el establecimiento definitivo del orden público; el mejoramiento de la libertad y de la educación política; la garantía de las libertades civiles; la economía en la administración, y el progreso sin interrupciones, de los intereses materiales del país", Idem, 22-23.

19 Hora (2005) XV.

20 Idem, XXII. Ver también: Halperin Donghi (1963). Garavaglia, Gelman (2003); Gelman (2005).

21 Idem, XXIV.

22 AgüERo (2011).

23 OszlaK (2011) 70. Botana (1998). Al respecto resulta sumamente ilustrativa la caracterización que José Nicolás Matienzo hace de los gobernadores provinciales: «Los gobernado- 
Un espacio privilegiado de este poder local y tradicional era la campaña, constituyendo la estancia el núcleo organizativo de la vida de ésta. En ellas se lograba el disciplinamiento de la fuerza de trabajo y el aprovechamiento integral del ganado, siendo a su vez unidad productiva y unidad políticosocial, que abarcaba «desde la organización militar necesaria para defenderse de los indios y para actuar como policía rural, hasta la producción de la mayor parte de los consumos internos». ${ }^{24}$

Desde el período colonial el mundo rural estuvo regido por una serie de costumbres que se proyectaron más allá de la independencia. Las mismas, que en general emanaban de los cabildos, se referían a variados aspectos de la vida social y económica de la campaña, como el disciplinamiento de la población rural, el control de la vagancia, la regulación de las vaquerías, el uso del agua, de los bosques, pastos y rastrojos, el trabajo rural, así como la regulación de la propiedad tanto inmueble como del ganado. ${ }^{25}$ Durante el período patrio pervivieron sin modificaciones sustanciales, receptándose en los distintos códigos rurales que las provincias sancionaron durante el período de organización nacional.

Este orden consuetudinario era el que reflejó Valentín Alsina al redactar el texto del Código Rural de la Provincia de Buenos Aires, sancionado en 1865. Como recuerda Carlos Storni, Alsina no encontraba en la legislación extranjera «modelo a imitar», basando su proyecto en las disposiciones diseminadas en el Registro Oficial, en su propia experiencia en la materia y en las opiniones vertidas por la Comisión de Hacendados, ${ }^{\mathbf{2 6}}$ que representaba los

res argentinos son mucho más poderosos que los norteamericanos, canadienses y australianos ... Pero la preocupación principal de los gobernadores de provincia, salvo escasas excepciones, no es gobernar, sino mandar. La manera usual de expresar que un gobernador ha tomado posesión de su cargo es decir que ha asumido el mando. La Constitución escrita no emplea esta expresión. Para el texto constitucional, lo que el gobernador ejerce es el Poder ejecutivo; pero para la Constitución real y para el público, lo que ejerce es el mando, como en la época colonial ... Y por mando entienden en primer término el derecho de imponer su propia voluntad a los demás en todos los asuntos en que las facultades legales o la influencia del gobernador puedan intervenir, $y$, muy especialmente, en los asuntos políticos y electorales ... El gobernador se considera director de la política provincial y único autorizado para decidir la actitud de la provincia respecto del presidente de la República», Matienzo (1917) 196-199.

24 Oszlak (2011) 49.

25 StORni (1997a).

26 STORni (1997b) 196. Un estudio pormenorizado de las respuestas a esta consulta a la Comisión de Hacendados en Amaral (1998) 143-148. 
intereses terratenientes. Incluso su revisión, dos años más tarde, estuvo sometida a la consulta que se efectuara a la Sociedad Rural Argentina. ${ }^{27}$ De ahí, este instrumento normativo que pretendía "cortar los abusos e inconvenientes que retardan el progreso", se hacía eco de un derecho y poder tradicionales. $^{28}$

Si bien el progreso normativo al medio rural llegaría de la mano del dictado de leyes especiales ${ }^{29}$ lo cierto es que las codificaciones rurales mantuvieron vigente el orden antiguo que ni el código civil ni las interpretaciones modernizantes pudieron socavar.

El Código Rural de la Provincia de Buenos Aires, recibió del derecho indiano un régimen ancestral de propiedad del ganado, ${ }^{30}$ que se efectivizaba mediante el sistema de marcas y señales. ${ }^{31}$ Se ha hecho notar que el mante-

27 Asamblea General Extraordinaria de la Sociedad Rural Argentina, celebrada el 23 de agosto de 1867; Nota del Presidente de la Sociedad Rural Argentina, José Martínez de Hoz, al Gobernador de Buenos Aires, de fecha 23 de julio de 1869.

28 Así lo entiende Storni: «El silencio que guarda en relación con los antecedentes coloniales es, a mi juicio, sólo problema declarativo o de enunciación o exposición, pues ¿qué sentido puede darse a su persistente consulta a la Comisión de Hacendados? Es sin duda una apelación a la tradición, a los usos rurales y a la práctica, lo que atempera aquel racionalismo que siempre subyace en su pensamiento", STORni (1997b) 199. También señala Agüero que el contenido normativo esté determinado por los hacendados «pone en evidencia la persistencia de un ideal de autorregulación corporativa de hondo arraigo en la cultura jurisdiccional de antiguo régimen» donde «la intervención legislativa se concibe como una acción dirigida a mantener una idea de orden social mediante la satisfacción de un interés particular (aunque de un sujeto colectivo) más que a imponer estándares comunes y abstractos sobre los individuos genéricamente considerados», AGüEro (2011), la cita corresponde a la página 7. Con respecto a su valor normativo se ha señalado que «en este Código se observa claramente la incidencia de la costumbre, al ser instituidas antiguas tradiciones (por ejemplo, el derecho de rodeo y de tránsito con animales), y buscar el equilibrio entre los intereses de los grandes propietarios ganaderos (reflejados en la defensa de la propiedad privada y en la imposición de la obligación de cerramiento de los campos) y los menos poderosos», Barandiarán (2011). Ver también Zeberio (20052006).

29 Por ejemplo la ley 3965 (1902) sobre Sanidad Animal, 11.170 (1921) sobre contratos agrarios, etc.

30 Storni (1997a).

31 Mientras que la marca es «tanto el dibujo que constituye el signo con que se individualiza y distingue el dominio de los semovientes en el ganado mayor, como el signo o dibujo estampado en aquéllos - los textos legales refieren el vocablo únicamente a esta acepción -, y también - en la comprensión rural - el instrumento de hierro que lleva ese signo o dibujo y con el cual se lo estampa en el animal (fierro de marcar)»; la señal es «el signo de 
nimiento de esta práctica fue una de las cuestiones que mayor interés atrajo entre los estancieros consultados por Alsina. ${ }^{32}$

El artículo 17 de este código establecía que «la marca indica y prueba acabadamente, y en todas partes, la propiedad del animal u objeto que la lleva», entendiéndose que establecía una presunción iuris et de iure de dominio a favor del propietario de la marca. ${ }^{33}$

Sancionado el Código Civil, se fue imponiendo la opinión que la normativa contenida en la legislación rural contradecía lo dispuesto por el artículo 2412 del código civil, ley nacional de fondo, según el cual «la posesión de buena fe de una cosa mueble, crea a favor del poseedor la presunción de tener la propiedad de ella, y el poder de repeler cualquier acción de reivindicación, si la cosa no hubiese sido robada o perdida», y que en este sentido era inconstitucional en razón que usurpaba una potestad reservada al Congreso de la Nación en virtud de lo establecido por los artículos 67, inc. 11 y 108 de la Constitución Nacional. ${ }^{34}$ Esta doctrina mayoritaria entendió que la «sola propiedad de la marca o señal no bastaba para justificar el dominio del ganado y que la posesión establecía a favor del poseedor un título suficiente acerca de la legitimidad de la misma, sin perjuicio de admitir la prueba en contrario». Pero también se argumentó «que era suficiente presunción y prueba del dominio sobre un animal el hecho de tener de buena fe la posesión del mismo, fuera de quien fuere la propiedad de la marca». Y frente a la colisión normativa se sostuvo que debía prevalecer la disposición de carácter nacional y «para que la posesión confiriera el derecho de dominio ella debía ser de buena fe, de acuerdo con el art. 2412 del Código Civil, interpretándose que en materia de semovientes no hay buena fe si no existe la marca y señal correspondiente o el certificado de transferencia». ${ }^{35}$

propiedad que se hace en el ganado menor - y a veces en el mayor - cercenando o cortando de maneras determinadas y convencionales una parte de la oreja, o mediante tajos especiales en la piel, que se registraban como señales de propiedad y podían usarse en combinación con las señales de orejas», Соцомво (1998) 322-323 у 348.

32 Amaral (1998) 146.

33 Esta doctrina, denominada «tradicionalista» fue seguida por los códigos de Catamarca (1878), Salta (1884), San Luis (1888), Jujuy (1893), Territorios Nacionales (1894) y Tucumán (1897). La tesitura contraria, que permitía la prueba en contrario, fue seguida por los códigos de Mendoza (1880), Córdoba (1885), Entre Ríos (1882), Santiago del Estero (1897), Santa Fe (1901) y Corrientes (1902). Ver Colombo (1998) 367.

34 Martínez Golletti (2005).

35 Соцомво (1998) 368-369. 
La jurisprudencia, tanto de la provincia de Buenos Aires como la de los tribunales nacionales, lejos de declarar la inconstitucionalidad de la normativa rural, hizo un intento de armonizar los distintos preceptos, dando preferencia a la aplicación de la presunción del citado artículo del Código Civil; pero por otro lado no podría invocarse buena fe si se poseyera un animal con una marca ajena. ${ }^{36}$

En definitiva, la reconocía que la aplicación de la norma civil resultaba antieconómica y restaba fuerzas a la protección de la propiedad del ganado, y a la lucha contra formas delictivas que atentaban contra ella, como el abigeato. $^{37}$

Si la dispoción del código civil resultaba inconveniente, tampoco el mantenimiento de los sistemas locales implicaba una solución. Resulta interesante observar que, frente a la falta de un régimen legal unificado sobre marcas y señales, fue la misma Sociedad Rural Argentina la que tomó cartas en el asunto, organizando en 1898 un concurso de marcas y señales. Las condiciones del mismo consistían en la presentación de un proyecto de sistema de señales para ganado vacuno $u$ ovino, uno de marcas a fuego para ganado mayor y otro de sistema de administración pública de ambos. Y era requisito esencial que dichos sistemas fueran adaptables para su empleo en toda la República. La Sociedad Rural Argentina se comprometía a presentar los proyectos aceptados ${ }^{38}$ al gobierno pidiendo su adopción mediante una ley del Congreso. ${ }^{39}$

Diversos intentos legislativos trataron de dar una solución definitiva al asunto. En primer lugar encontramos, en 1890, el Proyecto de Código Rural para la Provincia de Buenos Aires, redactado por Manuel Gonnet, que aspiró armonizar ambos ordenamientos, estableciendo en su artículo 490 que la «marca indica y prueba acabadamente y en todas partes, la propiedad del ganado mayor salvo prueba en contrario». ${ }^{40}$ En 1910, en el proyecto redactado por los juristas Matías Sanchez Sorondo y Marcos A. Avellaneda, éstos entendían que las legislaciones provinciales no podían alterar el principio del

Ver jurisprudencia citada por Colombo (1998) 369-370; También: Garbarini Islas (1925) 231-232; LAFAILle (1943) 519.

37 Yangilevich (2009).

38 Fueron aceptados los proyectos de los señores A. Montes y J.C. Blanco sierra, el primero para marcas y el segundo para señales, SAENZ (1906) 53 ss.

39 SAENz (1906) 54.

40 Gonnet (1890) 129. 
art. 2412 del código civil, correspondiendo solo a la Nación establecer el régimen de la propiedad; las provincias solo podían pretender la reglamentación de la prueba de la posesión de buena fe, siendo la marca el signo visible de ésta. ${ }^{41}$

De la misma época fue el proyecto de ley nacional redactado por el Subsecretario del Departamento de Agricultura, doctor Mario Sáenz, siendo Eleodoro Lobos ministro de Agricultura y que el Poder Ejecutivo envió al Senado en agosto de 1911. El mismo creaba un registro nacional de marcas y señales, estableciendo que «la marca o la señal registrada justifica la propiedad de los ganados que la llevan». Dicho proyecto no tuvo sanción legal. ${ }^{\mathbf{4 2}}$

El tema también fue contemplado en el Proyecto de Reforma al Código Civil, donde se recepta, como veremos, la doctrina mayoritaria. El artículo 1536 del proyecto disponía que «la marca o señal probará la propiedad del ganado mayor y menor que las llevare, en beneficio de quien las tuviese registradas», mientras que el artículo 1537 mandaba que «la transferencia del dominio de los ganados, convenida entre el enajenante y el adquirente, deberá realizarse por la inscripción en el registro». ${ }^{\mathbf{4 3}}$

La Cuarta Conferencia de Abogados de 1936, dedicó un espacio para la deliberación de la materia. Las argumentaciones giraron en torno a la necesidad de la unificación del sistema de marcas y señales, los pormenores de la transmisión del ganado, el problema del cuatrerismo y las atribuciones de la nación y de las provincias para organizar el registro correspondiente, siendo éste uno de los puntos más álgidos. Para algunos el avance de los poderes nacionales constituía un menoscabo de los provinciales:

«No debemos permitir que en los grandes asuntos, primero, y en los pequeños después, se vaya poco a poco produciendo el encerramiento de las facultades de las provincias, porque ello nos lleva a la disminución de la capacidad impositiva de las mismas, empobrece su economía y establece una dependencia con el gobierno federal - la dependencia económica - que es el medio más permanente y eficaz para sobornar las autonomías provinciales». ${ }^{44}$

Para otros, «el gobierno nacional ha sido creado para los fines tendientes a la unidad de esfuerzo y del progreso general»:

41 Serrés (1957) 21.

42 Garbarini Islas (1925) 229-230; Lafaille (1943) 520.

43 Reforma del Código Civil (1936) 597-598.

44 Cuarta Conferencia Nacional de Abogados (1936) 198. 
«Si tuviéramos que aceptar ... esta teoría que pretende difundir el localismo y que resucitaría las viejas cuestiones de antaño, tendríamos que suprimir el cúmulo de manifestaciones de progreso que produce la Nación, como obras públicas, patentes y caminos, colegios nacionales, escuelas normales, escuelas Láinez, etcétera. Tendríamos que cerrar los ojos ante este luz civilizadora difundida por todos los ámbitos de la República y que ha traído el desarrollo cultural de la Nación». ${ }^{45}$

Finalmente se aprobó una declaración que afirmaba: «a) Que la marca o la señal acredita la propiedad originaria de los ganados. b) Que debe adoptarse un sistema único de marcación y señalamiento para todo el país. Que la marca y la señal deberán inscribirse en los registros que a tal efecto crearán la Nación y las provincias». ${ }^{46}$

La solución legislativa definitiva llegó siete décadas después, en 1983, con la ley 22.939 que crea el registro nacional de marcas y señas. ${ }^{47}$

\section{El debate académico}

La cuestión hasta aquí reseñada suscitó un interesante debate académico ${ }^{48}$ que giró en torno a la validez constitucional de los ordenamientos rurales y a la posibilidad de «compatibilizar» las disposiciones del código civil con las de aquéllos.

Fue en los claustros de la Facultad de Derecho y Ciencias Sociales, en las cátedras de Derecho Civil y Legislación de Minas, rural e industrial, donde se esbozaron los primeros argumentos. ${ }^{49}$ Los testimonios en este sentido son

45 Cuarta Conferencia Nacional de Abogados (1936) 188 y 200.

46 La comisión que trabajó en el proyecto de declaración estaba integrada por los doctores Rodolfo Arnado, Máximo Castro, Juan P. Danuzzo Amadey, Guillermo Garbarini Islas, Demetrio Morales y José Rafael Serres. Cuarta Conferencia Nacional de Abogados (1936) 430.

47 Cima, Martínez, Ventura, Moisset de Espanés (1984).

48 El término académico es utilizado aquí con el peso y profundidad que ha señalado en varias oportunidad Petit: «... el verbo académico nos sirve además como ejemplo de discurso corporativo, asunto éste de la voz de la corporación con derivaciones inmediatas en cuanto atañe al contenido de los discursos, a su censura y a la propiedad intelectual o 'literaria' de que pudieran ser objeto». Petit (2000) 25.

49 La estrecha relación entre enseñanza académica y producción del discurso jurídico ha sido señalada por Petit en la obra antes citada donde clases, lecciones inaugurales, discursos de colación de grado, que constituían «el saber de ese jurista y el derecho que de continuo le interesa, atrapados luego en textos que hoy constituyen las fuentes que facilitan su conocimiento» pero que «fueron en una vida anterior tan sólo palabras pensadas para ser dichas y oídas». Petit (2000) 11-12. 
coincidentes: las enseñanzas de los civilistas David Texanos Pintos a fines del siglo XIX y de Juan A. Bibiloni, dieron forma a la doctrina «conciliatoria» de las legislaciones civil y rural para el tema de las marcas y señas, doctrina que luego se proyectaría en la manualística y en la jurisprudencia de los tribunales. ${ }^{50}$ Ecos de la misma resonarán luego, como veremos, en la novel Facultad de Ciencias Económicas que tan estrecha relación tendrá en sus orígenes con aquélla casa de estudios. ${ }^{51}$

Si miramos los actores que participan en la producción de los distintos argumentos esgrimidos en el debate, nos encontraremos con personajes que no solo tuvieron un lugar destacado en el ámbito académico y jurídico, sino también en el quehacer político y económico, ocupando lugares claves en el gobierno y la administración de pública, ${ }^{52}$ algunos de ellos con conexiones directas con aquella institución que representaba los intereses ganaderos: la Sociedad Rural Argentina. ${ }^{53}$

\section{a) Villiers Tapia y Roth: una copia a ciegas de un sistema desastroso}

A instancias del que fuera catedrático de la recién creada cátedra de Derecho Rural, ${ }^{54}$ Eleodoro Lobos, los alumnos Leopoldo Villiers Tapia y Carlos Roth, publican en 1903 una obra escolar que titularon «Legislación Rural Argentina». La misma tenía como finalidad «facilitar el estudio de una materia tan importante como descuidada: el Derecho Rural». ${ }^{55}$

50 «Dentro del propio mecanismo del Código Civil, el doctor David de Tezanos Pinto, enseñaba en su cátedra desde fines del siglo XIX, que era posible conciliar ambas legislaciones, teniendo presente que el art. 2412 exige la buena fe y que no sería dado invocarla a quien poseyera un animal con distintivo ajeno. Fue del mismo parecer el doctor Juan Antonio Bibiloni, tanto en sus clases, como al ser consultado por la Suprema Corte de la Provincia de Buenos Aires, cuya jurisprudencia inspiró acerca de este problema», LAfaILle (1943) 518-519. Ver también Cima, Martínez, Ventura, Moisset de Espanés (1984).

51 Sobre el origen de esta Facultad y sus relaciones con la de Derecho y Ciencias Jurídicas ver: Fernández López (2001); Caravaca, Plotkin (2008).

52 Zimmermann (1999), especialmente 114-116. Stagnaro (2012).

53 Guillermo Garbarini Islas fue miembro de su Comisión Directiva, Sánchez SAÑudo (2000). Bibiloni no era la primera vez que intervenía a pedido de la SRA: ver BibiLonI, Saenz Peña, de la Torre (1904).

54 SAENZ (1906) 64.

55 Villiers Tapia, Roth (1903), «Introducción». 
En su Quinta Parte, los autores abordaban el problema de la «Propiedad de los semovientes», partiendo del análisis del sistema consagrado por el código civil para las cosas muebles en general. El mismo era considerado «desastroso» en su aplicación a la propiedad del ganado:

«Hay que tener en cuenta el medio, no es lo mismo probar en un centro poblado donde hay todos los elementos a mano y, donde es más difícil robar que en el campo donde no hay esos elementos, y la misma movilidad de los animales facilita la operación. Hay que ver la cantidad enorme de ganados y sus semejanzas que muchas veces impide distinguir un grupo de animales de otro, la riqueza fabulosa que para nuestro país representa la ganadería, para comprender el enorme descuido no disculpable puesto que existía ya el Código Rural de la Provincia de Buenos Aires y todos los antecedentes nacionales sobre marcas; nada se tuvo en cuenta, se copió a las autores franceses que legislaban en un país donde los animales están identificados individualmente y existen en una relación exigua a la de nuestro pais en cuanto a su cantidad, alli cada animal tiene su nombre y no puede confundirse; se ba copiado a ciegas y sin saber lo que se hacia». ${ }^{56}$

Por el contrario, las normas rurales tenían un profundo arraigo en la realidad nacional y así lo demostraban la extensa reseña de los antecedentes que desde 1817 realizan los autores.

¿Sería posible «conciliar» ambos ordenamientos? La conclusión que se esboza era positiva, a pesar de la «rigidez» de las leyes y de la Constitución, que disponía que solo el Congreso Nacional podía legislar sobre la propiedad y sus medios de adquisición a través del código civil, mientras que los códigos rurales «no pueden ni deben tener fuerza de ley». ${ }^{57}$ Superando esta interpretación, ambas legislaciones podían «coexistir», entre otras razones, porque los códigos rurales trataban una materia especial reglamentándola, "porque tiene sus peculiaridades que el Código Civil no ha podido considerar, viene a ser algo así como una ley que modificase la estricta rigidez de los principios generales». ${ }^{58} \mathrm{Y}$ agregaban:

«... visto el vacío del Código en materia de propiedad de ganado y siendo necesario salvarlo de cualquier modo, es necesario aceptar como «modus vivendi», las modificaciones que a los principios generales introduce el Código Rural, así como las modificaciones de hecho que en materia de transmisión de inmuebles se ha introducido a la

56 Villiers Tapia, Roth (1903) 273-274. El subrayado es nuestro.

57 Villiers Tapia, Roth (1903) 278. Los autores tuvieron presente el trabajo de Mario Saenz, que sería defendido como tesis tres años después. A pesar de ello, se apartarían, como puede observarse más adelante, de la posición de Saenz.

Villiers Tapia, Roth (1903) 278. El subrayado es nuestro. 
legislación civil por las leyes orgánicas de los Tribunales de la Capital y de algunas provincias, al imponer inconstitucional e ilegalmente que es necesaria la inscripción en los registros para que la transmisión de la propiedad sea válida contra terceros». ${ }^{59}$

Esta línea argumentativa resulta sumamente elocuente en varios sentidos. En primer lugar, deja entrever el espacio constitucional que se le asignaba al código civil como expresión de los principios rectores del orden civil, que se sustentaba en una interpretación exegética o «rígida» del mismo, propia de la época. A pesar de ello, los autores no tienen reparos en saltear ese orden, calificado, en el punto que nos ocupa, como una «mala copia», que había olvidado ese otro, tradicional y rural, donde se desarrollaba la principal actividad económica del país. De ahí que fuese necesario «salvar de cualquier modo» ese «vacío» de nuestra legislación, aceptando como «modus vivendi» aquel orden normativo que la codificación había relegado al lugar de la «inconstitucionalidad» y de la «ilegalidad». Así también la propuesta para regularizar la situación: la reforma del código civil estableciendo un régimen especial para los semovientes adaptado a las normas rurales y la creación de un sistema único de marcas y señales. ${ }^{60}$

\section{b) Mario Sáenz: nadie mejor que el propietario sabe lo que le conviene}

En 1906 se publicaba la tesis de Mario Saenz titulada «Propiedad de Ganados», proponiendo la reforma de la legislación vigente a fin de que se reglamente por separado este tipo de propiedad. ${ }^{61}$ Partía para ello de la reformulación de los principios del código civil que regían la propiedad mueble. A la distinción de las cosas en muebles e inmuebles, este autor sugería una distinción tripartita que contemplara a los semovientes separados de los muebles. Fundamentaba esta clasificación en el derecho romano, ya que era este derecho fuente de principios para la misma codificación civil. Entendía que

59 Villiers Tapia, Roth (1903) 278. El subrayado es nuestro.

60 Villiers Tapia, Roth (1903) 293-294. Sigue en esto la solución, como se verá, de Mario Saenz. La respaldan con el proyecto de marcas y señales del presidente de la Sociedad Rural.

61 SAEnz (1906) 15. Esta no es la primera tesis que aborda la cuestión. En 1881, Francisco Ayerza esboza la tesis de la posibilidad de conciliar el ordenamiento civil y rural. Ver SAEnz (1906) 48. Dicha tesis se publicó en 1881 (Averza 1881). Existen copias en la Biblioteca Nacional y en la Facultad de Derecho de Buenos Aires, pero por razones técnicas alegadas por ambas bibliotecas en material no se encontraba disponible. 
«Desgraciadamente, en este punto, los legisladores de las sociedades modernas, en vez de inspirarse en la vida del Derecho Romano, han transcripto la letra de sus preceptos, la han calcado servilmente, en un momento de su evolución, resultando así muy lejos de la verdad, por haber confundido con principios permanentes de derecho, los que no son sino elementos móviles de la legislación». ${ }^{\mathbf{6 2}}$

Era esta la razón por la cual «el codificador argentino, cuya erudición y talento han dado tan brillantes frutos, en otras secciones del código, han incurrido aquí en los mismos errores de los modelos que seguía». ${ }^{63}$ Remarcaba que las disposiciones del código civil sobre bienes muebles fueron «calcadas, en lo fundamental, sobre el código Napoleon» y que no se justificaba «que hayamos de aplicar a los muebles tales prescripciones, solo porque ellas rigen en Inglaterra o Francia». ${ }^{64}$ Luego, el artículo 2412 del código civil no satisfacía las necesidades del país, ni favorecía el desarrollo de la ganadería argentina:

«Se comprenderá que las disposiciones del Código Civil son tan deficientes para proteger la propiedad que ni ex profeso podrían ser mejor calculadas para alentar el robo». ${ }^{65}$

Ahora bien, si bien reconocía que las normas contenidas en los códigos rurales sobre esta materia eran «superiores al Código Civil, dado el medio en deben imperar», las mismas en la práctica han derivado en «grandes inconvenientes», debido a la regulación local de las marcas y señales, que posibilitaba que en una provincia se registraran iguales marcas que en otra. ${ }^{\mathbf{6 6}}$

La solución para Sáenz pasaba por la reforma del artículo 2318 del código civil, que separase al regulación de los semovientes del sistema general de las cosas muebles. ${ }^{67} \mathrm{Y}$ establecer un régimen especial para éstos, organizando un sistema único en toda la República adoptando el trabajo realizado por la Sociedad Rural Argentina «cuya preparación especialísima en estas materias reviste opiniones de gran autoridad y prestigio». ${ }^{68}$ Las normas debían también adaptarse a las nuevas exigencias desarrolladas por

62 SAenz (1906) 23-24.

63 SAENZ (1906) 24.

64 SAENZ (1906) 34-35.

65 SAENZ (1906) 34.

66 SAENZ (1906) 51.

67 Proponía suprimir de dicha normativa la frase «... sea moviéndose por sí mismos», SAENZ (1906) 60.

68 Se refiere al concurso de 1898. SAEnz (1906) 54. 
la actividad, y proteger a aquellos ganaderos que se preocupan por mejorar la raza o la pureza de sus haciendas, no imponiendo la obligación de marcar a sus animales. Es que después de todo, «a este respecto nadie mejor que el propietario sabe lo que le conviene». ${ }^{69}$

\section{c) Garbarini Islas: una inconstitucionalidad «catastrófica»}

En 1925, Guillermo Garbarini Islas ${ }^{70}$ publicaba su «Derecho rural argentino» ${ }^{71}$ que, dedicado «especialmente a los estudiantes», exponía, por primera vez en forma sistemática, los elementos de lo que él consideraba el más "típico y nacional de los derechos». ${ }^{72}$

En el prólogo de la obra, el entonces decano de la Facultad de Ciencias Económicas de Buenos Aires, José León Suarez, reconocía que «el Derecho Rural es una formación de nuestros tiempos ... una «consagración» reciente, puesto que siempre existió informe o implícito en el civil y en otros derechos» y se lamentaba de la «tardanza extraña en un país esencialmente rural» con la cual se había conformado esta nueva rama jurídica. En realidad lo que reclamaba era su atención académica «autónoma» en atención a las «necesidades modernas» y al «proceso económico del mundo», sin que ello obstara a que reconociese la jurisdicción federal sobre la materia rural, como si la autonomía disciplinar y poder federal fuesen dos caras de la misma moneda:

«La importancia de estas nociones es evidente en un país que ha discutido durante un siglo, sin llegar a ninguna resolución definitiva, sobre las facultades del gobierno federal y de los gobiernos provinciales en cuanto a sistemas de marcas y señales de ganado, con el propósito, impostergable, de evitar la confusión y el fraude a que hoy se presta la falta de un sistema y de una reglamentación únicos en la República. Por otra parte, se observa en la legislación de las provincias una verdadera anarquía de criterio en unos casos y una imitación servil en otros, en cuanto a la redacción de las disposiciones que pueden considerarse típicas de derecho rural, tales, por ejemplo,

69 SAENZ (1906) 63.

70 Se graduó de abogado en la Facultad de Derecho y Ciencias Sociales de la Universidad de Buenos Aires en 1922, obteniendo al año siguiente el título de doctor. Inicia su actividad como profesor universitario en 1923, desarrollando la misma en la Facultad de Ciencias Económicas de Buenos Aires, en la Facultad de Agronomía y Veterinaria de Rosario y en la Universidad de La Plata, destacándose como fundador de la Universidad Libre del Museo Social, en 1956, SánChez SaÑudo (2012).

71 Garbarini Islas (1923).

72 Garbarini Islas (1923) 5-6. 
como la clasificación y protección de los animales útiles, el ejercicio de la caza, etc., etc.» ${ }^{73}$

Si bien con el mismo fin, las argumentaciones de Garbarini Islas corrían por rieles diferentes. Para este autor, el «derecho rural no es un derecho autónomo, es, en su mayor parte, una rama del derecho civil» y por lo tanto reservado a la jurisdicción del Congreso Nacional en virtud de lo establecido por el art. 67, inc. 11 de la Constitución Nacional. ${ }^{74}$ Negar su autonomía era, en definitiva, afianzar el carácter nacional de este derecho.

En el trasfondo de esta interpretación, Garbarini Islas practicaba una profunda crítica a nuestro régimen federal. En una extensa nota sostenía su convencimiento que «entre nosotros tenga razón de ser el federalismo», que «solo existía en el papel», y servía "para mantener catorce Estados innecesarios dentro del Estado, con gobernadores, ministros, cámaras y mil funcionarios» que comían «el dinero de los productores sin beneficio alguno». El mismo constituía

«una copia bastarda de instituciones extranjeras, que por desgracia somos tan afectos a imitar sin entender, con frecuencia, su espíritu y que lo que es peor, sin darnos cuenta de las diferencias enormes que van desde los países que las crearon a nuestra patria, que tan a la zaga de ellos va en tantas cosas». ${ }^{75}$

Si el mismo podía tener razón de ser en Estados Unidos, no existía razón histórica ni política para sostenerlo en la Argentina:

«Convengo en que los constituyentes que hubieron de constituir un país cuando por todos lados ardía la hoguera anárquica, no el principio federalista!, hicieron bien en adoptar la forma gubernativa que adoptaron, y otra no pudieron implantar a fuer de hombres prácticos, pero hoy, después de medio siglo, cuando la locomotora y el telégrafo han substituido a la carreta y al chasque, cuando tras las tolderías del indio han venido las ciudades florecientes y tras el desierto los campos de cultivo y pastoreo; hoy, en que el cielo ha sido empañado por el humo de las fábricas y las

73 José León Suarez, «Prólogo», en Garbarini Islas (1923) 7.

74 Idem, 23. Esta interpretación a favor de la jurisdicción federal, que puede ser discutible, se basaba principalmente en el juego de los arts. 67, inc. 11 [actual art. 75, inc. 12: «Corresponde al Congreso ... dictar los Códigos Civil ...»] y el art. 104 [actual 121: Las provincias conservan todo el poder no delegado por esta Constitución al Gobierno federal, y el que expresamente se hayan reservado por pactos especiales al tiempo de su incorporación] y seguía la doctrina constitucionalista más tradicional de Juan Manuel Estrada y Montes de Oca a favor de los poderes nacionales. Ver Estrada (1895) 337-339; Montes de Oca (1903) 439 ss.

75 Idem 22, nota 11. 
montañas horadadas y los ríos canalizados en busca de mercados para nuestros productos, sólo falta después de haber reemplazado la vetusta legislación española por códigos modernos, completar la evolución en lo político y en lo moral para terminar la gran obra que nuestros padres emprendieron hace un siglo». ${ }^{76}$

Vuelve sobre estas ideas al referirse al sistema de marcas y señales diseñado en nuestro ordenamiento jurídico. Para Garbarini Islas, contrario a otras posturas ${ }^{77}$ el sistema seguía siendo adecuado para la realidad rural de nuestras pampas. Pero consideraba inconstitucionales las disposiciones que sobre esta materia dedicaban los códigos rurales de las provincias, materia reservada al Código Civil de acuerdo a lo dispuesto por el mencionado art. 67 , inc. $11 .^{78}$ Y si bien se hallaban en contradicción con el art. 2412 del Código Civil, que consagraba el principio de la presunción de propiedad del poseedor de buena fe, dicho principio no podía hacerse valer sin más

«en esas pampas inmensas en que se anda horas enteras sin ver un ser humano y a veces ni un mísero rancho en el horizonte y dígase si la sola posesión puede servir, como en Europa, para acreditar la propiedad. Bastaría el salto de un animal por arriba de un alambrado para que éste cambiando de campo hubiese cambiado de dueño; y aun los mismos propietarios procediendo de perfecta buena fe ¿podrían asegurar entre cinco o diez mil cabezas de ganado, a veces de idéntica mestización y pelaje, que éste o aquél les pertenece?» ${ }^{79}$

A la inconstitucionalidad de los ordenamientos provinciales, se sumaban razones de conveniencia que hacían indispensable una legislación nacional sobre señales y marcas: la existencia de numerosas marcas iguales registradas en diversas provincias, lo que facilitaba el abigeato. ${ }^{\mathbf{8 0}}$

Pero una afirmación, hecha al pasar, lleva al autor a reconocer el arraigo del ordenamiento provincial y la dificultad de declararlas inconstitucionales: «Imagínense, advierte, la catástrofe que sobrevendría si la Suprema Corte las declarase tales». ${ }^{\mathbf{8 1}}$ Era, en definitiva, la primacía de la realidad frente a la perfección del sistema y la congruencia de las normas esgrimida por el hombre de derecho. Esa conservación del ordenamiento dejaba entrever también en la jurisprudencia, que nuestro jurista reconoce como «pobre», ${ }^{\mathbf{8 2}}$ la cual sin

76 Ibidem.

77 Por ejemplo la sostenida por Ramos Mejía (1903) 973.

78 Garbarini Islas (1925) 223.

79 Garbarini Islas (1925) 224-225.

80 Garbarini Islas (1925) 223.

81 Ibidem.

82 Y esta pobreza resultaría también un indicio de la falta de cuestionamiento del sistema en la práctica. 
aplicar el remedio federal, se contenta simplemente con realizar algún tipo de armonización de la normativa rural con el principio consagrado en el art. 2412 del Código Civil. ${ }^{83}$

\section{d) Bibiloni y los altos intereses comprometidos en el Código Civil}

Una de las líneas argumentativas más importantes sería la que construyera Juan A. Bibiloni, importante civilista de la época, profesor titular de Derecho Civil y autor del Anteproyecto de Reforma del Código Civil, su mayor legado jurídico.

Su primer encuentro con la cuestión dataría de 1903, a instancias del entonces presidente de la Sociedad Rural Argentina, Ezequiel Ramos Mejía, quien sostenía que la «legislación sobre marcas y señales de los ganados es, y debe ser, de atribución federal», entendiendo que las disposiciones de los códigos rurales fueron derogadas con la sanción del código civil. ${ }^{84}$ Bibiloni da la razón a éste y entiende que las disposiciones de los ordenamientos rurales «no tienen fuerza de ley» y que la cuestión analizada debe ser resuelta dentro de los principios del código civil, ya que «en materia civil, no hay, pues, legislación supletoria». ${ }^{85} \mathrm{~A}$ su vez afirmaba:

«Que el régimen del Código Civil no sea satisfactorio, me parece evidente. Ninguna de las razones que se dan para justificar el principio que gobierna la posesión de cosas muebles, me parece aplicable en nuestro país, y dadas sus condiciones peculiares, a los ganados. Ningún interés social, de rapidez en la transmisión, de seguridad o estabilidad en la propiedad, aconseja a dispensar al poseedor de la prueba de su título. Creo al contrario, que existen razones que inducen a establecer el régimen opuesto». ${ }^{86}$

De allí que propiciara la reforma del Código Civil donde las bases del sistema fueran «las líneas fundamentales del régimen actual de hecho, que es el resultado de una experiencia ya secular, perfeccionándolas». ${ }^{87}$ No bastaba, por tanto la sola corrección del artículo 2412 del código civil, sino que debería establecer la propiedad dependiente del registro público que sería

83 Garbarini Islas (1925) 231-232.

84 Ramos Mejía (1903). Junto con sus consideraciones publica un proyecto de ley que de solución a la cuestión.

85 Bibiloni (1903).

86 Idem.

87 Idem. 
gobernado «en sus líneas principales por la ley del Congreso, uniforme para toda la Nación, y se entregaría a la jurisdicción de las provincias». ${ }^{88}$

Bibiloni tuvo la oportunidad de concretar su propuesta. En su Anteproyecto, se refirió al sistema de marcas y señales bajo el título «De la propiedad de ganados y máquinas locomóviles», ${ }^{\mathbf{9 9}}$ dedicándole tan solo seis artículos. ${ }^{\mathbf{9 0}}$ En la nota que dedica a la explicación de los mismos, reproduce los argumentos ya vertidos en su carta de 1903. Los preceptos propuestos tenían por objeto reconocer la valía de ese «hecho existente», como que eran los ordenamientos provinciales. Se imponía «regularizar legislativamente el derecho que diremos consuetudinario", costumbre que se remontaba a «los orígenes de la colonización de nuestro país» y que respondía a «las necesidades causadas por la cantidad de reses en los ganados, la que ha impuesto la marca como única solución, es decir la identificación colectiva, y no la individual». De esta manera lo establecía el artículo 2469: «Las leyes locales determinarán, las condiciones de la concesión de marcas y señales, y su empleo. Los registros quedarán sometidos a su reglamentación y bajo la dirección y vigilancia de las autoridades que esas leyes determinen». No era posible seguir en la materia a las legislaciones europeas que no consagraban el mismo principio, donde «la pequeña cantidad de los animales permite indi-

88 Idem.

89 El asunto se hallaba regulado en el Libro III, De los Derechos Reales, Título IV, De la propiedad de las cosas, Capítulo II, De la adquisición y pérdida de la propiedad de cosas muebles, $\mathbb{S} 5$, De la propiedad de ganados y máquinas locomóviles.

90 Art. 2463: La marca o señal prueba La propiedad Del ganado mayor o menor que La lleva, a favor de los que las tenían registradas a su nombre; art. 2464: La transmisión del dominio de los ganados convenida entre el enajenante y el adquirente se realiza por la inscripción del acuerdo en el registro. La ley no reconoce otra manera de transmisión por actos entre vivos, sin perjuicio de lo dispuesto sobre ejecución de sentencia; art. 2465: La marca y la señal debidamente registradas constituyen bienes exclusivos de las personas a quienes se hubieran concedido. Pueden transmitirlas, pero no pueden ser embargadas, ni ejecutadas por acción de los acreedores; art. 2466: Cuando se solicite la inscripción en el registro de un acuerdo de transmisión, se verificará e inscribirá el título del enajenante, si no estuviera ya inscripto. La inscripción equivale a título efectivo. Le es aplicable lo dispuesto por el artículo 2400; art. 2467: Cuando la señal o marca consistiese en tatuaje u otro medio especial no reglamentado por la ley, se anotará en el registro la declaración previa del propietario de que ella constituirá su manera de identificar los animales de la especie que determinará en la exposición firmada que se archivará en el registro; art. 2469: Las leyes locales determinarán, las condiciones de la concesión de marcas y señales, y su empleo. Los registros quedarán sometidos a su reglamentación y bajo la dirección y vigilancia de las autoridades que esas leyes determinen, BibILONI (1936) 113-118. 
vidualizarlos, como ocurre entre nosotros, con los de raza, o pedigree». ${ }^{91} \mathrm{Si}$ era aplicable, en cambio, el ejemplo de algunas legislaciones estaduales de los Estados Unidos, a las cuales nos unía un «tronco común» que era, en definitiva la «legislación española». ${ }^{\mathbf{2}}$

De esta manera, reconocido el sistema de marcas y señales por el Código Civil, se salvaba la cuestión federal, y se establecía cierto principio general que hacía a la legitimidad de dicho ordenamiento al imponer su inscripción: «La inscripción, en todo caso, es la condición fundamental de nuestro proyecto. ¿Se adopta marca?: se adquiere como las leyes locales lo autorizan, y bajo sus condiciones se efectúa la marcación, o señal en su caso. Para el Código Civil, lo esencial es la inscripción». ${ }^{93} \mathrm{Y}$ ahí terminaba la cuestión, aunque a Bibiloni la solución si le sabía a conveniente al estado actual de la actividad, no le resultaba del todo convincente:

«Debemos desear que este asunto sea materia, algún día, de la legislación nacional. No se conciben marcas provinciales, como no se conciben propiedades locales. Las garantías no pueden detenerse en los límites de cada distrito del país ... Mientras no se dicta una ley general, para las transmisiones de propiedad de ganados [...] es forzoso aceptar la situación actual, de las leyes provinciales. Muy grave es, pero no susceptible de ser corregida en un C. Civil, por el carácter en cierta manera reglamentario de cualquier ley que la naturaleza del asunto impone. Baste decir, que las marcas otorgadas libremente por cada provincia, permiten muy frecuentes actos de despojo de la propiedad ganadera. Con obtener la misma marca en otro distrito se pueden ejecutar impunemente. Agréguese que los embarques por ferrocarril, y los fluviales, dan grave actualidad a ese asunto, y según lo hemos aconsejado en otras oportunidades, imponen el estudio de un sistema nacional de marcas, y su aplicación, quedando, naturalmente, a las provincias su jurisdicción sobre los registros, y el poder reglamentario para establecer las condiciones de ejecución del sistema general». ${ }^{94}$

91 BibiLoni (1936) nota al $\$ 5$, pág. 114.

92 Ibidem. «En los Estados Unidos, en que algunos de los particulares, - California, Tejas, Wyoming, Colorado, Arizona, Utah, New Mexico, etc. - han practicado la cría en vasta escala, el mismo régimen de la marca existe, y la propiedad y transmisión de los ganados, se acredita por el título constituido o fundado en ella. Legislativamente no está esa clase de bienes dentro del régimen general de las cosas muebles. Hemos utilizado en nuestros proyectados artículos, no solamente las leyes de esos Estados, sino también las de nuestras provincias, que al fin salen de un tronco común, esto es el de la legislación española, pues en California, Tejas, Colorado, Nueva Méjico, etc., tiene ese origen la legislación vigente y bien se advierte a la primer ojeada».

93 Bibiloni (1936) nota al art. 2463, p. 117.

94 BibiLoni (1936) 115 y 117. 


\section{e) Lafaille y una dualidad tolerable}

«Dualidad de la legislación» era, para Héctor Lafaille, destacado civilista y profesor de la materia en la Facultad de Derecho de la Universidad de Buenos Aires, la característica principal de un régimen «cuyos antecedentes se remontaban a la época colonial», y que a pesar de la sanción del Código Civil «tal estado de cosas persiste, y las provincias consignan, como antes, en cuerpos de leyes especiales, el principio que lo inspira». ${ }^{95}$

Si bien su examen se centraba, fundamentalmente, en describir el régimen legal vigente sobre propiedad de los «semovientes», con el estudio de sus antecedentes y jurisprudencia, lo cierto es que nuestro jurista, que había también participado en la Comisión de Reforma del Código Civil, se hacía eco en su análisis de la doctrina mayoritaria, que como hemos visto, proclamaba la supremacía de la regla contenida en el código civil, que equiparaba la posesión de buena fe al título, por entender que el punto estaba comprendido en la esfera reservada al Congreso de la Nación, habiendo cesado cualquier derecho de los poderes locales desde la sanción del código. ${ }^{96}$ Sin embargo reconocía que la cuestión tenía sus límites y que era preciso mantener aquellas interpretaciones que conciliaran los dos ordenamientos:

«Por más que esta sea en rigor la doctrina justa, son tantos los intereses en juego y tan grande el perjuicio derivado de ella, que todos estimaron necesario encontrar un expediente, que permita reconocer eficacia a las marcas y a las señales, hasta la promulgación de una ley que desvaneciera la antinomia existente». ${ }^{97}$

Urgía, sin embargo, llegar a un criterio legal indiscutible, que pusiese «fin a la incertidumbre, en materia que tanto se relaciona con la vida económica

95 Lafaille (1943) 517 ss.

96 Idem, 518.

97 Ibidem. «Una de las soluciones es la de considerar incluido dentro del «poder de policía», que conservaron las provincias (art. 104 de la Constitución Nacional), el de promulgar aquellas normas necesarias para la mayor seguridad del dominio. No podrían ciertamente - se dice - legislar sobre la condición jurídica de los semovientes, ni declarar cómo y cuándo se adquieren o transmiten, pero los preceptos que analizamos podrían juzgarse complementarios de los establecidos por el Código Civil, como lo han sido los referentes a la propiedad inmueble. Se trata en rigor, de algo más que de simples detalles o reglamentos de seguridad, puesto que se afecta al título mismo. Además, la jurisprudencia - que se ha tornado rigurosa en punto a los registros - no podría resolver de otra manera, en el supuesto de plantearse la cuestión». 
del país». ${ }^{98}$ Reconocía que el régimen imperante respondía, en general a «las condiciones peculiares de la cría de ganado en los países hispanoamericanos» que tornaba inaplicables «los principios que gobiernan esta materia en la legislación europea». Luego, resultaba evidente la imposibilidad de adaptar a los semovientes el precepto del art. 2412 del Código Civil:

«La gran cantidad de cabezas de ganado impide que el dueño conozca individualmente a cada animal, a diferencia de cuanto acontece en otras regiones; a lo que se agrega la topografía del terreno y el deslinde por medio de alambrados, que permiten trasladar la hacienda de un fundo a otro y favorecen el abigeato. Estas condiciones agravan las circunstancias que concurren para los muebles en general - y obligan a valerse de medios adecuados, a fin de proteger el dominio». ${ }^{\mathbf{9}}$

El sistema de marca y señal cumplían acabadamente los propósitos de protección del derecho de propiedad sobre el ganado:

«Conviene, pues, mantener tal sistema; pero dotándolo de la uniformidad y sobre todo, de la legalidad, que le falta entre nosotros: lo primero, porque cada provincia y los territorios nacionales pueden tener $-\mathrm{y}$ de hecho, tienen a veces - regímenes diversos; fuera de que un mismo signo se repite a menudo, por hallarse inscripto en jurisdicciones independientes. Lo segundo, por las condiciones que se adujeron sobre el problema de la constitucionalidad».

En la argumentación jurídica ganaba, una vez más, las razones de conveniencia práctica sobre la razón legal pura y abstracta, y las necesidades de la vida económica del país determinaban la solución jurídica posible.

\section{Consideraciones finales}

Si gran parte de nuestras elites vieron en la sanción del código civil el cumplimiento de una exigencia del progreso, una refundación del nuestro ordenamiento jurídico en base a los principios de la revolución emancipadora, lo cierto es que el estudio de su aplicación concreta, la reconstrucción de la historia del código, como propone Pio Caroni, nos demuestra una realidad mucho más compleja y no tan idealista.

El código como puerta de entrada a la "civilización» implicaba de suyo descartar un espacio que no se identificaba ella: la pampa, el desierto, el lugar de la barbarie. Significaba también, por lo menos en la retórica con la cual

98 Ibidem, 519.

99 Ibidem. 
Vélez acompañó la presentación de su proyecto, dejar de lado tradiciones la obstaculizaban: «En la necesidad de desenvolver el derecho por la legislación, ya que nos falta la ventaja que tuvo el pueblo romano de poseer una legislación original, nacida con la nación, y con ella crecía, podíamos ocurrir al derecho científico, del cual pueden ser dignos representantes los autores citados». ${ }^{\mathbf{1 0 0}}$

La codificación civil supuso también la concreción de un ideal de uniformidad legislativa, que tenía su correlato en la paulatina consolidación del poder nacional. Pero éste tuvo que chocar con el contexto que dominaba nuestro país en el período estudiado.

Por un lado, el vigor de ciertos intereses corporativos, como el de los hacendados, que veían en la vigencia «fáctica» de los ordenamientos rurales, a pesar de su «ilegalidad»e «inconstitucionalidad», una protección más positiva de los mismos.

Por el otro, la disímil valoración de nuestro federalismo, que íbase redefiniendo a medida que el poder federal ganaba en recursos y atribuciones en detrimento de las provincias. Las argumentaciones dirigidas a conferir al Congreso Nacional la facultad exclusiva en materia rural, a pesar del cuestionado valor de las codificaciones provinciales, nos parece que es un ejemplo claro de ello. La preeminencia de las reglas contenidas en el Código Civil por sobre las normas provinciales, no era solo expresión del lugar medular que ocupaba este ordenamiento en la elaboración del discurso jurídico de la época, sino también la función conductiva de la legislación nacional en la construcción de un orden normativo más racional y unificado. Asimismo la tacha de inconstitucionalidad de dichos ordenamientos, lejos de aparecer como una mera postura doctrinal, legitimaba al Estado Nacional como el único dotado de los medios necesarios para llevar adelante el ideal de progreso.

100 El subrayado es nuestro. VéLez SARsfield (1869) VI. La cita se refiere a la nota que Vélez dirigió al Ministro de Justicia, Culto e Instrucción Pública, Dr. Eduardo Costa, con fecha 21 de junio de 1865 . 


\section{Bibliografía}

Agüero, Alejandro (2011), Tradición jurídica y derecho local en época constitucional: El «Reglamento para la Administración de justicia y policía en la campaña» de Córdoba, 1856, en: Revista de Historia del Derecho, Instituto de Investigaciones de Historia del Derecho, Buenos Aires, $n^{\circ} 41$, pp. 1-43 http://www. scielo.org.ar/pdf/rhd/n41/n41a01.pdf (consultado el 26/06/2012)

Alberdi, Juan Bautista (1852), Bases y puntos de partida para la organización política de la República Argentina (La primera edicíon fue publicada en Valparaíso, por la imprenta del Mercurio, en 1852. Las citas de este trabajo corresponden a la edición realizada en 1915 en Bueonos Aires por la Cultura Argentina.

Altamirano, Carlos (2008), Introducción General, en: Historia de los intelectuales en América Latina, I. La ciudad letrada, de la conquista al modernismo, Editor del volumen Jorge Myers, Buenos Aires, Katz, pp. 9 y sgtes

Amaral, Samuel (1998), The rise of capitalism on the pampas. The estancias of Buenos Aires, 1785-1870, Cambridge University Press

Asamblea General Extraordinaria de la Sociedad Rural Argentina, celebrada el 23 de agosto de 1867, en: Anales de la Sociedad Rural Argentina (1867), Primer volumen (desde septiembre de 1866 hasta diciembre de 1867), Buenos Aires, p. 402 ss.

Ayerza, Francisco (1881), Apuntes al estudio del Código Rural, Buenos Aires, M. Biedma

Barandiarán, Luciano O. (2011), La figura de la vagancia en el Código Rural de Buenos Aires (1856-1870), en: Quinto Sol, Vol. 15, № 1 http://ojs.fchst.unlpam. edu.ar/ojs/index.php/quintosol/article/viewFile/113/98 (consultado el 23/09/ 2013)

Bibiloni, Juan B. (1903), Carta del Dr. Bibiloni, en: Anales de la Sociedad Rural Argentina, Volumen XXXVIII, ${ }^{\circ}$ 7, 31 de julio, pp. 978-981

Bibiloni, Juan Antonio (1936), Reforma del Código Civil, Buenos Aires, Kraft

Bibiloni, Juan Antonio, Roque Saenz Peña, Calixto de la Torre (1904), Informe de los jurisconsultos. En la consulta de la Sociedad Rural Argentina sobre inconstitucionalidad del proyecto de ley de impuesto a la transacciones, Buenos Aires

Botana, Natalio (1998), El orden conservador. La política argentina entre 1880 y 1916, Nueva edición con estudio preliminar, Buenos Aires, Editorial Sudamericana

Botana, Natalio, Ezequiel Gallo (2007), De la República posible a la República verdadera (1880-1910), $2^{\mathrm{a}}$ ed., Emecé

Caravaca, Jimena, Mariano Plotkin (2008), Crisis, ciencias sociales y elites estatales: la constitución del campo de los economistas estatales en la Argentina, 1910-1935 http://saberesdeestado.ides.org.ar/files/2008/05/plotkin_caravaca.pdf (consultado el 23/09/2013) 
Caroni, Pio (2012), Constitución liberal y código civil en el siglo XIX, en: Escritos sobre la codificación, Universidad Carlos III de Madrid, http://e-archivo.uc3m. es/handle/10016/13028

Cima, Luis B. (h), Víctor H. Martínez, Gabriel Ventura, Luis Moisset de Espanés (1984), Propiedad de los Semovientes, en: Academia Nacional de Derecho y Ciencias Sociales de Córdoba, Doctrina, http:/www.acaderc.org.ar/doctrina/ articulos/artpropiedadsemovientes/?searchterm $=$ marcas $\% 20 \mathrm{y} \% 20 \mathrm{se} \% \mathrm{C} 3 \% \mathrm{~B} 1$ ales (consultado el 26/06/12)

Código de Comercio para el Estado de Buenos Aires, Buenos Aires, Librería C. M. Joly

Colombo, Guillermo (1998), Los signos del dominio del ganado (marcas y señales) en el derecho rural argentino, en: Revista de Historia del Derecho, Instituto de Investigaciones de Historia del Derecho, Buenos Aires, n 26, pp. 321-377

Cuarta Conferencia Nacional de Abogados, Tucumán Julio 13-18 de 1936. Programa. Antecedentes. Versión taquigráfica y Anexos, Buenos Aires

Dotti, Jorge (1990), Las vetas del texto. Una lectura filosófica de Alberdi, los positivistas, Juan B. Justo, Buenos Aires, Punto Sur Editores

Estrada, Juan Manuel de (1895), Curso de Derecho constitucional federal y administrativo, Conferencias dadas en la Universidad de Buenos Aires en los años 1877, 1878 y 1880, Buenos Aires, Compañía Sudamericana de Billetes de Banco

Fernández López, Manuel (2001), La ciencia económica argentina en el siglo XX, en: XXXVI Reunión Anual - Buenos Aires, organizada por UCEMA http:// www.aaep.org.ar/anales/works/works2001/fernandez-lopez.pdf (consultado el 23/09/2013)

Garavaglia, Juan Carlos, Jorge Gelman (2003), Capitalismo agrario en la frontera. Buenos Aires y la región pampeana en el siglo XIX, en: Historia agraria, $\mathrm{N}^{\circ} 29$, Abril, pp. 105-121

Garbarini Islas, Guillermo (1923), Régimen jurídico de la ganadería argentina: fragmento de una obra en preparación, Buenos Aires, Restoy \& Doeste

Garbarini Islas, Guillermo (1925), Derecho rural argentino, Buenos Aires, Librería de Derecho y Jurisprudencia Restoy \&Doeste

García CANClini, Néstor (2010), Culturas híbridas. Estrategias para entrar y salir de la modernidad, Nueva edición, 3era. reimpresión, Paidós

García Sebastiani, Marcela (2008), Interlocutores y escenarios del liberalismo reformista español en Argentina, en: Sebastiani, Marcela García, Fernando del Rey Reguillo (eds.), Los desafíos de la libertad. Transformación y crisis del liberalismo en Europa y América Latina, Madrid, pp. 349-372

Gelman, Jorge (2005), Derechos de propiedad, crecimiento económico y desigualdad en la región pampeana, siglos XVIII y XIX, en: Historia Agraria, n 37 , Diciembre, pp. 467-488

Halperin Donghi, Tulio (1963), La expansión ganadera en la campaña de Buenos Aires (1810-1852), en: Desarrollo Económico, Vol. III, Nº 1-2, pp. 57-110 
Gonnet, Manuel B. (1890), Proyecto de Código Rural e Industrial para La Provincia de Buenos Aires, Buenos Aires, El Censor

Hora, Roy (2005), Los terratenientes de la pampa argentina. Una historia social y política, 1860-1945, Buenos Aires, Siglo XXI

Lafaille, Héctor (1943), Derecho Civil, Tomo III, Tratado de los derechos reales, vol. 1, Buenos Aires, Compañía Argentina de Editores

Leiva, Alberto David (1988), El Código civil como objeto didáctico en la Argentina a fines del siglo XIX, en: Revista de Historia del Derecho, $\mathrm{n}^{\circ} 16$, Instituto de Investigaciones de Historia del Derecho, Buenos Aires, pp. 321-330

Martínez Golletti, Luis E. (2005), Marcas y señales, propiedad del ganado y la Constitución Nacional, en: Academia Nacional de Derecho y Ciencias Sociales de Córdoba, Doctrina T. XLIV (http://www.acaderc.org.ar/doctrina/articulos/ artpropiedaddelganado/?searchterm $=$ marcas $\% 20 \mathrm{y} \% 20 \mathrm{se} \% \mathrm{C} 3 \% \mathrm{~B} 1 \mathrm{ales}$ (consultado el 26/06/12)

Matienzo, José Nicolás (1917), El gobierno representativo federal en la República Argentina, 2a ed., Madrid

Montes de Oca, Manuel Augusto (1903), Notas tomadas de las conferencias del doctor Manuel Augusto Montes de Oca, Volumen II, Buenos Aires, Tipo Litográfica «La Buenos Aires»

Nota del Presidente de la Sociedad Rural Argentina, José Martínez de Hoz, al Gobernador de Buenos Aires, de fecha 23 de julio de 1869, en: Anales de la Sociedad Rural Argentina (1869), Tercer volumen (desde enero hasta diciembre de 1869), Buenos Aires, p. 249

Oszlak, Oscar (2011), La formación del Estado argentino. Orden, progreso y organización nacional, $2^{\text {a }}$ ed., Emecé

Palti, Elías (1998), Giro lingüístico e historia intelectual, Universidad Nacional de Quilmes

Palti, Elías (2007), Lugares y no lugares de las ideas en América Latina, en: El tiempo de la política. El siglo XIX reconsiderado, Siglo Veintiuno Editores, pp. 259-308

Palti, Elías (2008), La invención de una legitimidad. Razón y retórica en el pensamiento mexicano del siglo XIX (Un estudio sobre las formas del discurso político), Primera edición argentina, Fondo de cultura económica

Petit, Carlos (2000), Discurso sobre el Discurso. Oralidad y Escritura en la Cultura Jurídica de la España Liberal. Lección inaugural curso académico 2002-2001, Universidad de Huelva

Polotto, María Rosario (2012), Argumentación jurídica y trasfondo ideológico. Análisis del debate legislativo sobre prórroga de alquileres en Argentina a principios del siglo XX, en: Rechtsgeschichte, $\mathrm{n}^{\circ} 20$, pp. 309-327

Ramos Mejí́, Ezequiel (1903), La marca a fuego en el ganado, en: Anales de la Sociedad Rural Argentina, Volumen XXXVIII, nº 7, 31 de julio, pp. 973-977

Reforma del Código Civil (1936), I. Antecedentes II. Informe III. Proyecto, Buenos Aires, Gmo. Kraft Ltda. 
Rivarola, Rodolfo (1908), Del régimen federativo al unitario. Estudio sobre la organización política de la Argentina, Buenos Aires

Saenz, Mario (1906), Propiedad de ganados, Tesis presentada para optar al grado de doctor en Jurisprudencia, Universidad de Buenos Aires, Facultad de Derecho y Ciencias Sociales, Buenos Aires

Sánchez Sañudo, Carlos A. (2000), Homenaje a los académicos Guillermo Garbarini Islas y Héctor P. Lanfranco, disertación en sesión pública de la Academia Nacional de Ciencias Morales y Polticas, el 11 de octubre de 2000 http:// www.ancmyp.org.ar/user/files/homenajeagarbariniislas.pdf (consultado el 24/ 09/2013)

Schwarz, Roberto (1977), As ideias fora do lugar, en: Ao vencedor as batatas. Forma literária e processo social nos inícios do romance brasileiro, Librária Duas Cidades

Serrés, José Rafael (1957), Bienes Rurales. Régimen legal de la propiedad de ganados, de su transmisión y del tránsito, Buenos Aires, Academia Nacional de Agronomía y Veterinaria

Stagnaro, Andrés (2012), Vocación de poder. Los abogados porteños a través de las colaciones de grado. 1884-1919, en: Temas de historia argentina y americana $\mathrm{N}^{\circ} 20 \mathrm{http}$ //bibliotecadigital.uca.edu.ar/repositorio/revistas/vocacion-poderabogados-portenos-stagnaro.pdf (consultado el 23/09/2013)

Storni, Carlos Mario (1997a), Un derecho embrionario en la antigua Buenos Aires, en: Investigaciones sobre la historia del derecho rural argentino. Españoles, criollos, indios y gauderios en la llanura pampeana, Buenos Aires, Instituto de Investigaciones de Historia del Derecho, pp. 13-33

Storni, Carlos Mario (1997b), Notas sobre el código rural de la provincia de Buenos Aires de 1865, en: Investigaciones sobre la historia del derecho rural argentino. Españoles, criollos, indios y gauderios en la llanura pampeana, Buenos Aires, Instituto de Investigaciones de Historia del Derecho, pp. $193-214$

Tau Anzoátegui, Víctor (1982), Los comienzos de la fundamentación de las sentencias en la Argentina, en: Revista de Historia del Derecho, $\mathrm{n}^{\circ} 10$, Instituto de Investigaciones de Historia del Derecho, Buenos Aires, pp. 267-371

Tau Anzoátegui, Víctor (1998), La "cultura del código»: un debate virtual entre Segovia y Sáenz, en: Revista de Historia del Derecho, $\mathrm{n}^{\circ}$ 26, Instituto de Investigaciones de Historia del Derecho, Buenos Aires, pp. 539-566

Tau Anzoátegui, Víctor (2008), La codificación en la Argentina, 1810-1870. Mentalidad social e ideas jurídicas, $2^{\mathrm{a}}$ ed. revisada, Buenos Aires, Librería-Editorial Histórica Emilio J. Perrot

Velez Sarsfield, Dalmacio (1869), Proyecto de Código Civil para la República Argentina, Buenos Aires, Imprenta Pablo Coni

Velez Sarsfield, Dalmacio (1920), El folleto del doctor Alberdi, en: Cabral Texo, Jorge (comp.), Juicios críticos sobre el Proyecto del Código Civil, Buenos Aires, Jesús Mendez Editor, 1920, 233-256 (Artículo publicado en El Nacional 
el 25 de julio de 1868 y en la Tribuna el 29 de julio del mismo año. La cita se toma de la obra que compila estos textos.)

Villiers Tapia, Leopoldo, Carlos Roth (1903), Legislación Rural Argentina, Buenos Aires, Imp. Europea de M. A. Rosas

Yangilevich, Melina (2009), Normas, rupturas y continuidades: La administración de justicia y los ataques contra la propiedad en la provincia de Buenos Aires ( $2^{\mathrm{a}}$ mitad del siglo XIX), en: Revista de Historia del Derecho, Instituto de Investigaciones de Historia del Derecho, Buenos Aires, n. 38, dic. http:// www.scielo.org.ar/scielo.php?script=sci_arttext\&pid=S1853-17842009000200005\&lng=es\&nrm=iso (accedido en 26/09/2013)

Zeberio, Blanca (2005-2006), Los hombres y las cosas. Cambios y continuidades en los Derechos de propiedad (Argentina, Siglo XIX), en: Quinto Sol, № 9-10, pp. 151-183 (online) (consultado el 23/09/2013)

Zimmermann Eduardo (1998), El poder judicial, la construcción del estado y el federalismo: Argentina, 1860-1880, en: Posada-Carbó, Eduardo (ed.), In search of a New Order: Essays on the Politics and Society of Nineteenth-Century Latin America, The Institute of Latin American Studies, University of London, pp. 145 y sigtes.

Zimmermann, Eduardo (1999), The education of Lawyers and Judges in Argentina's Organización Nacional (1860-1880), en: Zimmermann, Eduardo (ed.), Judicial Institutions in Nineteenth-Century Latin America, London, Institute of Latin American Studies, School of Advanced Study University of London, pp. 116-122

Zimmermann, Eduardo, Universidades, ciencias sociales, y política pública en la Argentina del primer Centenario: del conocimiento ilustrado al nuevo "conocimiento social», http://saberesdeestado.ides.org.ar/files/2008/05/eduardoazimmermann2.pdf (consultado el 23/09/2013) 

Mario G. Losano

\section{Tra Uruguay e Italia: \\ Couture e Calamadrei, due giuristi democratici nell'epoca delle dittature europee}

1 La circolazione delle idee giuridiche italiane:

l'esempio dell'Argentina

Le idee circolano per il mondo perché portate da persone, quindi le impronte europee in Sudamerica si rivelano anche attraverso le biografie dei disseminatori di idee. In particolare, «la storia degli intellettuali» è vista da Norberto Bobbio «come la storia della coscienza che i produttori e gli agitatori d'idee hanno del proprio tempo». ${ }^{1}$ Cercherò quindi di fornire qualche cenno biografico su alcuni giuristi che trasportarono idee europee in Sudamerica, o recepirono in Sudamerica idee che venivano dall'Europa. Stabilire la genealogia di certe concezioni giuridiche e la loro circolazione nel mondo è difficile e, spesso, incerto. Inoltre le origini, le radici, si rivelano talora fuorvianti. Tuttavia una cosa è certa: le comuni radici europeo-continentali, $\mathrm{e}$ in particolare iberico-romanistiche, facilitarono l'immediata acclimatazione in Sudamerica dei giuristi costretti all'esilio dalle dittature europee.

In rari casi, la recezione è chiara, quando il giurista funge da mediatore culturale: è il caso di Tobias Barreto (1839-1889), mulatto brasiliano che non fu mai in Germania, ma che introdusse la cultura giuridica tedesca in Brasile. ${ }^{2}$ In altri casi, pur essendo noto il vincolo con la cultura europea (in questo caso, italiana), il giurista ha una formazione plurale, ed è quindi difficile stabilire quale apporto culturale sia in lui dominante: è il caso di Miguel Reale (1910-2006), ${ }^{3}$ uno dei maggiori filosofi del diritto latino-americani del secolo scorso.

In altri casi ancora, l'essere europei di seconda generazione non implica necessariamente la funzione di mediatore delle idee europee o specificamente

1 Bobbio (1990) 209.

2 Per ogni informazione rinvio al mio volume Losano (2000b).

3 Losano (2006). 
italiane: Juan Antonio Bibiloni (1860-1933) giurista e ministro della giustizia in Argentina, era di origine spagnola, ma, nonostante il cognome, aveva una formazione francese e tedesca. Poco dopo la sua morte un suo conterraneo ricordava che «sin apartarse de sus maestros franceses, que le fueron tan caros, aprendió ya en plena madurez el alemán», e sono «los autores germanos» quelli «que tanta influencia habrían de tener en su anteproyecto», ${ }^{4}$ cioè nel suo progetto di codice civile argentino.

Uno dei più fortunati articoli dell'esportazione culturale italiana fu la Scuola Positiva italiana. Cesare Lombroso, Enrico Ferri e Raffaele Garofalo, seguiti dalla Scuola Critica di Emanuele Carnevale, conobbero un'ampia diffusione in America Latina e, in particolare, in Argentina, a Buenos Aires, all'Universidad de la Plata e a Córdoba: «El positivismo argentino, surgido contemporaneamente al italiano, en vez de ser una mera copia, tuvo rasgos de verdadera originalidad». Inoltre il positivismo si avverte «en los proyectos de código y de ley que siguieron al de 1891, hasta el Código Penal de 1922». ${ }^{5}$ Un'antologia dei positivisti argentini venne pubblicata nel $1985 .^{6}$

La cultura argentina retroagì anche su quella italiana, come dimostra la prefazione di Lombroso a un libro curato anche da Francisco Ramos Mejía e pubblicato a Torino, l'allora capitale del tardivo positivismo italiano. Nella sua prefazione, Lombroso definisce Mejía «uno dei più potenti pensatori e dei più grandi alienisti dei due mondi», che ha rivelato «le relazioni fra genio e pazzia, mostrando come quasi tutti i capi e grandi rivoluzionari della repubblica Argentina erano stati o pazzi, o alcoolisti o neuropatici» (p. XXXI). Lombroso traccia poi un panorama della fortuna del positivismo nell'America del Sud (pp. XXXI-XXXV), spiegandone la diffusione con le contrapposizioni senilità/gioventù e pazzia/genio: «Le nuove idee sorte nella vecchia Europa dovranno morirvi isterilite», conclude, mentre «troveranno nel Nuovo Mondo chi le perpetuerà fecondandole ed applicandole» (p. XXXIX). ${ }^{7}$

4 Toвal (1939): volume che raccoglie articoli di quotidiano, come ad esempio Bibiloni y el anteproyecto, 19-34; i passi citati sono a p. 22.

5 Levaggi (1978); le citazioni sono a p. 209 e 211. A p. 210 è ricordata la criminologia di Pietro Gori e, a p. 212, la critica al positivismo che si sviluppò in Argentina con Manuel Obarrio e Lozano (1889).

6 Biagini (1985).

7 Drago (1890), in particolare, Francisco Ramos Mejía, Introduzione, 1-8, Cesare LomBRoso, Sulla diffusione della antropologia criminale, V-LXXIX. 
A volte l'influenza del positivismo italiano e, in generale, della cultura europea era indiretta: per esempio lo storico alicantino Rafael Altamira y Crevea (1866-1951), dell'Università di Oviedo, visitò l'Argentina poco dopo Enrico Ferri e scrisse un resoconto del suo viaggio del 1911 in America. ${ }^{8}$ Quest'ultimo viaggio è oggetto di un'analisi di Ernesto Quesada. ${ }^{9}$ Un'altra fonte indiretta di circolazione delle idee europee (non solo italiane, quindi) furono gli esuli spagnoli della Guerra civile, come il celebre Luis Jiménez de Asúa. ${ }^{10}$

Il diritto penale fu influenzato anche dalle idee classiche italiane, dall'opera di Mondolfo su Beccaria ${ }^{11}$ sino a Francesco Carrara, la cui opera principale venne presentata da Francisco Laplaza in una conferenza presso la società «Dante Alighieri», in occasione della prima edizione completa in spagnolo del Programa del curso de derecho criminal. ${ }^{\mathbf{1 2}}$ Laplaza fu anche autore di ampie rassegne sulle riviste giuridiche argentine. ${ }^{13}$

Il maggior filosofo del diritto argentino, Carlos Cossio, si richiama a molti autori italiani: non solo Norberto Bobbio (autore destinato a una vasta diffusione futura in Sudamerica; ${ }^{\mathbf{1 4}}$ L'analogia nella logica del diritto, 1938), ma anche Giovanni Brunetti (Completezza e incompletezza dell'ordinamento giuridico, «Rivista Internazionale di Filosofia del Diritto», 1925, p. 607); Giuseppe Capograssi (Il problema della scienza del diritto, 1937); Umberto Campagnolo (La notion de «personne juridique» dans la Doctrine pure du droit, nella kelseniana «Revue Internationale de la Théorie du Droit»); Tommaso Castiglia (Stato e diritto in Kelsen, Torino 1936); Donato Donati (Il problema delle lacune, 1910); Vincenzo Miceli (Il dogma della completezza dell'ordinamento giuridico, «Rivista Internazionale di Filosofia del Diritto», 1925); Renato Treves (Il diritto come relazione, 1934, e Id., Il problema dell'esperienza giuridica e [...] Schuppe, 1938); nonché molte opere di Benedetto Croce e di Giorgio Del Vecchio. ${ }^{15}$

8 Levaggi (1977) 91 (volume di 118 pp.); Altamira y Crevea (2007).

9 Quesada (1908).

10 Jiménez de Asúa (1960); JimÉnez de Asúa (1944) 1944 (contiene anche: El derecho penal de los indios, 251-257). Cfr. Mattes (1977).

11 Mondolfo (1946).

12 Laplaza (1950b).

13 Laplaza (1951); Laplaza (1950a). Inoltre, Laplaza (1954), il cui indice dei nomi è una miniera di indicazioni sulla circolazione delle idee penalistiche.

14 Filippi/Lafer (2006); Cardim (2001).

15 Cossio (1939): i nomi citati nel testo compaiono nella bibliografia. 
Anche in Brasile il positivismo italiano ebbe vasta diffusione. ${ }^{16}$ Un nome italiano che attira l'attenzione è quello di Clóvis Beviláqua (1859-1944), ${ }^{\mathbf{1 7}}$ autore del codice civile brasiliano rimasto in vigore dal 1916 al 2002, filosofo positivista e iniziatore degli studi di diritto comparato in Brasile. ${ }^{18}$ Questo celebrato giurista veniva da un famiglia avventurosa, anzi, quasi di avventurieri. Il nonno, Angelo, veniva dall'Italia, naufragò sulla costa cearense e lì mise radici. Il padre di Clóvis Beviláqua era un religioso che convisse lungamente con una donna, da cui ebbe anche altri figli. Un biografo di Clóvis Beviláqua propone una serie di luoghi originari della famiglia Beviláqua, pare nell'Italia del Nord, ma i legami originari con l'Italia appaiono evanescenti: l'unica certezza è che questo giurista è un brasiliano di origine lusobrasiliana e, per un quarto circa, italiana. ${ }^{19}$ La sua cultura giuridica è di origine europea, ma è difficile stabile quale sia l'apporto prevalente.

Quanto si è fin qui sommariamente accennato attesta l'importanza del contributo italiano alla cultura giuridica sudamericana e dà un'idea del campo di indagine che si apre a chi voglia studiare la circolazione delle idee giuridiche fra i due continenti. Tuttavia, per evitare un'eccessiva frammentazione, è opportuno concentrarsi ora su una figura centrale nella mediazione culturale novecentesca fra Europa e America del Sud: il processualista uruguaiano Eduardo J. Couture (1904-1956), uno dei pochi giuristi sudamericani contemporanei ricordato (in una voce scritta da Wolf Paul) nel dizionario biografico curato da Michael Stolleis. ${ }^{20}$ Grazie al suo aiuto trovarono

16 VAREjÃo (2005).

17 Una sintesi biografica è in STOLleis (1995) 85 s. Inoltre: Losano (2002); ripreso anche nel Prefácio do autor à tradução brasileira - $O$ direito sul-americano e os grandes sistemas jurídicos: a contribuição de Clóvis Beviláqua, in: Losano (2007) XV-XL.

18 Clóvis Beviláqua pubblicò a Recife nel 1893 un Resumo das lições de legislação comparada sobre o direito privado, come dispensa per gli studenti. La seconda edizione è un vero e proprio libro: Liç̧ões de legislação comparada, 1897. Beviláqua pubblicò poi $O$ desenvolvimento do estudo da legislação comparada, 1903; As funções da legislação comparada, 1906. Nei suoi scritti di diritto internazionale privato sottolinea l'importanza della comparazione: «O direito internacional privado exige, como preparo, o confronto das leis dos países que a civilização pôs em contato quotidiano, para saber-se quando elas coincidem ou divergem. E em que cadeira pode ser mais regularmente organizado este preparo senão naquela que tem por alvo direto a comparação das leis privadas?» (Beviláqua [1897] 12). Cfr. inoltre i suoi Princípios elementares de direito internacional privado (1906, ristampati 1978), nonché l'articolo Direito internacional privado, 1935.

19 Meira (1990). Informazioni biografiche alle pp. 33 ss.

20 STOLLeis (1995) $141 \mathrm{~s}$. 
accoglienza in America del Sud, tra molti altri, Renato Treves, ${ }^{\mathbf{2 1}}$ Enrico Tullio Liebman e Tullio Ascarelli. Come intellettuale ebbe un intenso scambio epistolare con il giusfilosofo italiano Giorgio Del Vecchio. ${ }^{22} \mathrm{Ma}$, soprattutto, fu legato da una profonda amicizia, da un idem sentire, con Piero Calamandrei, anch'egli processualista. ${ }^{23}$ Infatti Couture, benché legato alla cultura francese anche per le sue origini famigliari, si sentiva particolarmente vicino alla "pléiade di veri maestri» della scuola processualistica italiana: al fondatore Chiovenda, all'innovatore Carnelutti e soprattutto a Calamandrei, di cui ammirava «la vocazione dello scienziato, la formazione dell'umanista e l'austerità del cittadino». ${ }^{24}$

\section{Un archivio giuridico contemporaneo: l'archivio Couture a Montevideo}

L'esilio provocato dal fascismo e dalle leggi razziali sconvolse la vita di molti intellettuali italiani; tuttavia oggi è difficile immaginare in che modo essi abbiano potuto organizzare materialmente e culturalmente questa svolta radicale della loro esistenza. Alcune lettere inedite, trovate a Montevideo, possono aiutare a comprendere come alcuni Maestri milanesi - fra cui il sociologo del diritto Renato Treves e il processualista Tullio Liebman vissero quella transizione. Ripercorrere oggi quelle carte è una lezione di solidarietà e un bell'esempio delle "amicizie operose» di cui parla Piero Calamandrei. Ma è anche un esempio di come sia necessario coltivare la storia contemporanea del diritto, se non si vogliono perdere documenti importanti: tema su cui ritornerà l'ultimo paragrafo di questo scritto.

Devo premettere che da anni ho intrapreso lo studio di queste vicende con una profonda partecipazione personale, perché Renato Treves è stato il mio Maestro milanese, Enrico Tullio Liebman fu mio professore e poi collega a Milano, mentre i miei contatti con Tullio Ascarelli furono soltanto indiretti, perché mediati dal mio Maestro torinese Norberto Bobbio e dal-

21 Postscriptum rioplatense. Eduardo J. Couture e l'emigrazione intellettuale italiana a Montevideo, in: Losano (1998) 43-52; anche in Losano (1999); inoltre: Losano (2001b).

22 Losano (2001a).

23 Losano (2000a).

24 Così si esprime Couture nel prologo all'edizione in spagnolo del volume di CalamandreI (1936), citato da Treves (1957) 471. 
l'incontro con la sua biblioteca e con la sua eredità culturale nell'Università dello Stato di São Paulo (USP) in Brasile.

Lo studio delle carte conservate a Montevideo è nato dall'intersecarsi d'una mia ricostruzione del pensiero di Renato Treves, ${ }^{25}$ emigrato per tutta la durata della guerra a Tucumán, con il dibattito sulla storia contemporanea del diritto. ${ }^{26}$ L'interesse dei colleghi uruguaiani per queste ricerche ha poi avuto un'importanza decisiva nel far nascere, a Montevideo, un «Grupo de trabajo para la preservación de archivos jurídicos uruguayos», sotto gli auspici della Facoltà di Giurisprudenza dell'Universidad de la República. ${ }^{27}$ Infatti queste ricerche hanno dimostrato che gli archivi sono l'alimento della nostra memoria, ma che meriterebbero una maggiore cura istituzionale.

Nel 1938 Renato Treves, allora professore incaricato a Urbino, a causa delle leggi razziali venne escluso da un concorso universitario che avrebbe quasi certamente vinto. Prevedendo quale futuro gli avrebbe riservato l'Italia, nell'ottobre del 1938 s'imbarcò a Napoli e, dopo diciotto giorni di navigazione, sbarcò in Uruguay. Una lettera del penalista torinese Eugenio Florian lo presentava al collega uruguaiano Carlos Salvagno Campos, che a sua volta lo mise in contatto con il processualista Eduardo Couture, una figura eminente nella Facoltà di Giurisprudenza di Montevideo.

Ho avuto l'occasione di vistare la casa di Couture, la sua biblioteca e il suo archivio, apertimi dalla cortesia della sua famiglia. In particolare, alcune lettere scritte negli anni difficili intorno al 1938 mostrano quanto generoso sia stato l'appoggio accordato da questo studioso ai colleghi italiani in difficoltà.

Della professione d'avvocato Couture conservava anche nella vita accademica l'abitudine di tenere in ordine le carte: grazie alle cure della famiglia Couture, sono quindi ben conservate non solo le lettere, ma anche gli appunti di viaggio (raccolti in diari dattiloscritti e illustrati con fotografie) e le fotografie, raccolte in alcune scatole.

25 Losano (1998).

26 Losano (1997).

27 «En la sesión del 6 de agosto 1998 el Consejo de Facultad, con el decidido apoyo de la Decana Teresa Gnazzo, dicta la resolución $n^{\circ} 23$, por la cual se crea un Grupo de Trabajo y Seguimiento del plan de preservación acordado con el Prof. Losano. Dicho Grupo de Trabajo quedó integrado con los Sres Dr. Adolfo Gelsi Bidart, Américo Plá Rodriguez, Enrique Véscovi, Jorge Gamarra, Oscar Sarlo, Marcelo Bauzá, la bibliotecaria Nydia Bruzzese y el Prof. Mario Losano como miembro de honor» (SARLo [1998]). 
Di Couture si è già menzionata la vicinanza alla «pléiade di veri maestri» della scuola processualistica italiana. Dal canto suo, Calamandrei sottolineava così l'insostituibile attività di mediazione culturale di Couture: «Egli aveva per la scienza giuridica italiana una appassionata predilezione; insieme con Niceto Alcalá Zamora profugo dalla Spagna a Città del Messico, e con Santiago Sentís Melendo profugo in Argentina, Couture fu il presentatore e il divulgatore nell'America latina della letteratura processuale italiana». ${ }^{\mathbf{2 8}}$

Fin dai primi contatti scientifici, pur senza conoscersi personalmente, Couture e Calamandrei avvertirono una forte affinità elettiva, sentendo «di appartenere ad una stessa patria di pensieri e di affetti». ${ }^{29}$ Nel corso degli anni, fra i due si sviluppò un intenso scambio scientifico. ${ }^{30}$ Quando nel 1949, per la prima volta, Couture visitò l'Italia «quasi in pellegrinaggio», fu Calamandrei a dischiudergli le meraviglie di Firenze e della Toscana, in un incontro di cui ci resta oggi una testimonianza incrociata. ${ }^{31}$ Un secondo, rapido incontro nel $1953 \mathrm{fu}$ contrassegnato da un contributo di Couture alla rivista di Calamandrei ${ }^{32}$ e dal progetto di incontrarsi più a lungo nell'ottobre del 1956. «In quell'occasione, - scrive Calamandrei, - avrebbe dovuto inaugurare lui con un discorso l'Istituto di diritto processuale comparato, fondato nella rinnovata sede della facoltà giuridica fiorentina per rafforzare gli scambi scientifici tra i processualisti italiani e quelli iberoamericani». ${ }^{33}$ Incontro tragicamente impossibile, poiché entrambi morirono proprio in quel 1956, Couture l'11 maggio e Calamandrei il 27 settembre.

Calamandrei (1956) 247.

30 Per esempio, al volume in memoria di Calamandrei (che è, al tempo stesso, un fascicolo del «Boletín del Instituto de Derecho Procesal», 1957, n. 7, 175 pp., di quell'università), Homenaje a Piero Calamandrei. Introducción de Santiago Sentís Melendo, Universidad Nacional del Litoral, Santa Fe 1957, Couture partecipò con due scritti: La escuela italiana de derecho, 31-42, e Prólogo a la edición argentina del "Elogio de los jueces escrito por un abogado", 25-29. Questo prologo venne tradotto in italiano da Mauro Cappellettr: Eduardo Couture, Due libri in uno, in: Rivista di diritto processuale, XII, 1957, 64-67. Calamandrei ha lasciato un ricordo di Couture (CAlamandrei [1956]) conclusa da un breve elenco di «scritti specialmente dedicati alla letteratura processualistica italiana».

31 Calamandrei (1956) 247 s.; Couture (1953).

32 Couture (1954).

33 Calamandrei (1956) 248. 


\section{Dall'Europa all'Uruguay: le «amicizie operose»}

Negli anni delle dittature europee e della guerra queste «anime ardenti e comunicative» erano in piena attività. Fu Piero Calamandrei a segnalare a Couture alcuni studiosi italiani che, a causa delle leggi razziali, erano costretti a emigrare e cercavano quindi una sistemazione in Sudamerica. Simmetricamente, poi, lo stesso Calamandrei segnalava ai colleghi italiani costretti all'esilio le possibilità che Couture apriva loro in America Latina. Questa mediazione umanitaria e culturale traspare chiaramente dalla prima delle due lettere di Enrico Tullio Liebman, processualista anch'egli come Calamandrei e Couture.

Illustre Prof. Couture,

Il Prof. Calamandrei mi ha passato la Sua lettera [dell'] 8 ottobre u. s., in cui Ella manifesta tutto il Suo interessamento per il prof. [Alessandro] Pekelis. Questi ha intanto deciso di rimanere in Europa, e perciò vorrei pregarLa di fare per me quello che Ella si proponeva di fare per lui.

Infatti, sebbene io sia cattolico dalla nascita e figlio di madre cattolica, devo in forza degli ultimi provvedimenti lasciare l'insegnamento universitario in Italia.

Sono da sette anni professore ordinario di diritto processuale civile, che ho insegnato prima all'Università di Modena, poi in quella di Parma. Ho insegnato inoltre a Modena anche il diritto amministrativo e a Parma anche il diritto internazionale. Le mie opere principali sono: Le opposizioni di merito nel processo di esecuzione, Roma, 1. ed. 1931, 2. ed. 1936; Efficacia ed autorità della sentenza, Milano 1935; e numerosi articoli in tutte le riviste giuridiche italiane. Nel 1937 ho partecipato al II Congresso di diritto comparato dell'Aja, in qualità di relatore generale insieme con il prof. Giuseppe Chiovenda, mio compianto Maestro, e vi ho presentato e illustrato personalmente la relazione generale sul tema delle «Prove».

Sebbene non mi attenti di scriverLe ora questa lettera nella Sua lingua, posso dirLe tuttavia che parlo correntemente la lingua spagnola, avendo trascorso alcuni anni della mia infanzia in Spagna.

Forse questa circostanza potrà aiutarmi a svolgere l'insegnamento in un'Università americana e spero di poter operare a vantaggio della scienza e della gioventù studiosa anche al di là dell'Oceano, come fino a oggi ho operato nel mio paese. Certo per ottenere una sistemazione definitiva ci vorrà del tempo e la mia presenza presso di Voi. Ma spero che Ella possa intanto farmi avere al più presto possibile qualche incarico universitario e secondario (nel senso da Lei accennato nella Sua lettera), che mi consenta di provvedere alle prime necessità della vita, dato che non potrò espatriare se non con una piccola somma di denaro. Inoltre per ottenere il visto al passaporto, è necessario che possa dimostrare di avere un posto nel paese di destinazione. Le rivolgo perciò la più fervida preghiera di volersi interessare in questo senso. 
Nella speranza di fare presto la conoscenza Sua e dell'ambiente scientifico del Nuovo Mondo, mi professo, illustre Collega, con la più cordiale devozione. Suo obbl.mo

E. Tullio Liebman. ${ }^{34}$

L'incoraggiante risposta di Couture dovette giungere in pochi giorni, perché la seconda lettera di Liebman venne spedita dopo neanche due settimane dalla prima:

Illustre Prof. Couture,

Rispondo a volta di corriere alla gentilissima Sua lettera del 30 novembre u.s. e Le esprimo anzitutto la mia vivissima riconoscenza per la Sua cortesia che mi ha profondamente commosso e per l'aiuto valido e concreto che Ella mi porge nella presente circostanza.

Prendo nota di quanto mi comunica e non ho bisogno di aggiungere che impiegherò tutta la mia diligenza e buona volontà per mostrarmi degno della simpatia e dello spirito di colleganza che mi offre la Facoltà di Diritto di Montevideo.

In risposta alle Sue domande, Le dirò che sono certamente in grado di fare anche qualche insegnamento secondario, specialmente in filosofia, storia, pedagogia, lingua e letteratura italiana, francese e tedesca. Verrò in un primo momento da solo, ma col desiderio vivissimo di farmi raggiungere appena possibile da mia moglie, che ho sposato solo cinque mesi fa.

Il mio proposito sarebbe di imbarcarmi a febbraio allo scopo di prendere un poco di contatto con l'ambiente e rinfrescare l'esercizio della lingua spagnola prima dell'inizio dei corsi. Perciò mi pare che sarebbe opportuno che Ella mi facesse avere al più presto possibile la designazione ufficiale della Facoltà di diritto, sempre che ciò non debba esserLe eccessivamente gravoso, allo scopo di poter avviare senz'altro le pratiche per il passaporto e per ottenere il permesso di portare con me i mezzi necessari per vivere nel periodo iniziale della mia permanenza.

La relazione al Congresso dell'Aja sul tema delle Prove è in corso di stampa nel volume degli Atti del Congresso che si pubblicano a Parigi per cura del Segretario dell'Accademia di Diritto Comparato, Prof. Balogh. Le invio perciò in piego separato e in via riservata la copia che io ne ho (relazione speciale e relazione generale), con preghiera di volermela in seguito restituire, non avendone io altre copie (1).

$\mathrm{Al}$ più presto Le spedirò anche le mie principali pubblicazioni, che La prego di accogliere come omaggio e come pegno di particolare gratitudine e profonda stima. Di Lei devotissimo

Liebman

(1) Forse la relazione generale può interessare i lettori della rivista giuridica che Ella dirige ${ }^{35}$ In questo caso, se Ella ha il modo di farne fare la traduzione in spagnolo, io

34 Enrico Tullio Liebman a Eduardo J[uan] Couture, Milano, 22 novembre 1938, 2 pp. dattiloscritte; lettera conservata nell'archivio privato della famiglia Couture, Montevideo.

35 Liebman si riferisce alla «Revista de derecho, jurisprudencia y administración». 
sarei lieto e onorato di iniziare in questa forma la mia collaborazione alla letteratura giuridica uruguaiana. ${ }^{36}$

Liebman era destinato a insegnare in Brasile, in quell'Università di São Paulo che è un po' la mia alma mater brasiliensis e in cui il suo ricordo è ancora ben vivo, insieme con quello di Tullio Ascarelli: entrambi vi hanno lasciato una scuola, e Ascarelli anche la sua biblioteca. I suoi libri più rari sono ora conservati in una sala speciale della biblioteca della Facoltà di Giurisprudenza di Largo São Francisco.

Poiché le pagine seguenti conterranno soltanto qualche cenno su Ascarelli, vorrei ricordare che io stesso ho ancora potuto vedere alcune sue brevi lettere conservate dall'oggi defunto tributarista di São Paulo Rui Barbosa Nogueira, che era stato allievo e, poi, amico Tullio Ascarelli. Inoltre nel 1949 Ascarelli raccolse i suoi ricordi brasiliani in un libro destinato ai non giuristi. ${ }^{37}$

Infatti negli anni difficili del dopoguerra era ripresa l'emigrazione degli italiani e l'editore Giuffrè aveva affidato ad Ascarelli ed a Riccardo Bauer una collana intitolata «Paesi stranieri» per fornire un primo orientamento sui paesi cui si dirigevano gli emigranti. Il primo volume della collana fu proprio quello di Ascarelli sul Brasile: un'esposizione dunque volutamente di basso profilo, un libro d'informazione generale e divulgativa, ma documentato e ancor oggi attendibile come fonte storica su un Brasile che non c'è più.

Ascarelli giunse in Brasile già uomo maturo, quindi «è con mentalità europea che ho osservato il paese, mentre la maggior parte dei libri dedicati al Brasile sono stati scritti da autori nordamericani». ${ }^{38}$ Ascarelli aveva in Brasile un nucleo di allievi affezionati e di amici stretti, a due dei quali Noé Azevedo e Carlos Pinto Alves ${ }^{39}$ - dedicò il libro destinato ad aprire le

36 Enrico Tullio Liebman a Eduardo J. Couture, Milano, 5 dicembre 1938, 2 pp. dattiloscritte con tre righe ms. aggiunte dalla moglie, Adriana Liebman Gherardi (che però non seguì il marito in Sudamerica); lettera conservata nell'archivio privato della famiglia Couture, Montevideo.

37 Ascarelli (1949). L'ultimo saggio del volume di Bobbio (2007), 186-237, è dedicato ad Ascarelli: cfr. sul Brasile le pp. 207 ss.

38 AsCarelli (1949) 7.

39 L'amico Celso Lafer dell'Università di São Paulo (USP) mi ha cortesemente fornito queste informazioni di prima mano sulle persone cui Ascarelli dedicò il volume: «Noé Azevedo foi um ilustre professor de Direito Penal da Faculdade de Direito, um grande advogado e presidente, durante muitos anos, da Ordem dos Advogados do Brasil, Seção de São Paulo. Era um humanista e uma pessoa de larga visão. Fui seu aluno e ele foi advogado do meu 
porte del Brasile ad altri italiani, costretti all'emigrazione da condizioni di vita troppo dure, anche se meno drammatiche di quelle conosciute dalla generazione di Ascarelli.

Dei dodici capitoli nessuno è dedicato al diritto, anche se due tracciano un quadro politico dell'epoca successiva all'«Estado Novo» di Getúlio Vargas. ${ }^{40}$ Tuttavia, ammette Ascarelli, «come avvocato non posso mancare di dedicare in questo sguardo sommario qualche cenno al diritto brasiliano». Perciò egli riassume in due pagine l'influenza nordamericana sul diritto costituzionale (temperata da quella «francese nella filosofia politica»), mentre il diritto privato è «avvicinabile ai diritti dell'Europa continentale», con la peculiarità della «naturale persistenza nel diritto privato e processuale di concetti, istituti e tradizioni del diritto romano comune», dovuto all'«ininterrotto vigore delle ordinanze filippine del sec. XVII». ${ }^{41}$ Ricorda infine che i codici penale e di procedura penale erano «largamente influenzati dai rispettivi codici italiani» coevi. Ma lasciamo ora l'Ascarelli post-bellico per ritornare all'emigrazione pre-bellica dei giuristi italiani.

Mentre le lettere del 1938 di Liebman segnano l'inizio di una peregrinazione, una lettera del 1939 di Renato Treves annuncia invece una prima conclusione delle sue peripezie di esule: vi descrive infatti l'inizio di quella sua sistemazione a Tucumán che doveva durare sino alla fine della guerra.

Prima di passare in Argentina, Treves era rimasto «alcuni mesi in Uruguay, conquistando la simpatia e la stima di quanti lo avevano conosciuto»: così si legge nel 1939 sulla rivista di Couture, in una recensione del suo libro su Schuppe pubblicato l'anno prima a Milano. ${ }^{42}$ A questo punto sono necessari

avô. Carlos Pinto Alves foi um homem de grande presença intelectual em São Paulo. Era um católico com sensibilidade social, foi casado com uma artista plástica com atuação no cenário cultural de São Paulo, Mussia Pinto Alves. Todos os que com ele conviveram nele identificavam uma personalidade de escol, um homem de bem, um Ariel, como disse Alceu Amoroso Lima. Foi contemporâneo e amigo do meu tio, Horácio Lafer, na Faculdade de Direito. Em síntese, seguramente, tanto Noé Azevedo quanto Carlos Pinto Alves deviam ter sido amigos de qualidade humana e intelectual de Ascarelli, com os quais conviveu no seu período de Brasil» (e-mail dell'11 maggio 2013).

40 Ascarelli (1949) Cap. VIII, L'organizzazione politica, 154-167, e Cap. IX, Le classi politiche e i partiti, 168-180. Ascarelli scrisse il libro nel 1949, cioè nell'intervallo tra la fine dell'Estado Novo, nel 1934, e l'elezione di Vargas come Presidente della Repubblica nel 1951.

41 Ascarelli (1949) 212 s.: è il $\$ 11$ del capitolo su Pubblica istruzione e correnti culturali.

42 La recensione (Revista de derecho, jurisprudencia y administración, 1939, tomo XXXVII, 160) è firmata con la sigla «J. LL. A.»: con ogni probabilità, come mi ha indicato il 
alcuni chiarimenti, perché le notizie su quei mesi uruguaiani sono spesso vaghe e contraddittorie. Anzitutto, Treves si fermò vari mesi a Montevideo, almeno dalla fine dell'ottobre 1938 al febbraio 1939. A quell'epoca, inoltre, egli non aveva ancora una famiglia e quindi - a differenza, per esempio, di Liebman - non doveva risolvere il problema del ricongiungimento con i suoi famigliari.

Nell'ambito di queste precisazioni fattuali, è forse opportuno ricordare che Treves costituì la sua famiglia in Argentina. Nel febbraio del 1938 i giornali uruguaiani annunciavano il passaggio per Montevideo di un altro professore italiano costretto all'esilio: Leone Lattes, professore di medicina legale all'università di Pavia. Il rilievo dato alla notizia era dovuto anche al fatto che Leone Lattes era nipote di Cesare Lombroso (per la precisione, Lombroso era suo zio per parte materna). Lattes viaggiava con tutta la sua famiglia alla volta di Buenos Aires. Fu così che Treves, recatosi a salutarli nel breve intervallo a Montevideo del loro viaggio, incontrò la figlia di Leone, Fiamma Lattes. La rivide poi più volte in Argentina e, infine, la sposò a Buenos Aires nella libertaria ricorrenza del 14 luglio 1941. Ma fino a quella data le esistenze di Fiamma e di Renato si svolsero in luoghi diversi.

Rimasto a Montevideo dopo la partenza della famiglia Lattes, Treves pubblicò nella stessa annata della rivista di Couture anche l'articolo sul neohegelismo italiano e sul neokantismo tedesco, tratto da una conferenza tenuta nella Facoltà di Giurisprudenza di Montevideo. ${ }^{43}$ Insomma, Couture e i colleghi della facoltà si adoperarono in ogni modo per facilitare l'inserimento di Treves nel nuovo ambiente scientifico. Tuttavia Treves non poté restare all'università di Montevideo unicamente a causa delle retribuzioni troppo basse, non certo per la mancanza di disponibilità dei colleghi. È quindi naturale che, dopo un congresso a Buenos Aires e l'incontro con Carlos Cossio, Treves desiderasse informare anche Couture della stabilità finalmente raggiunta.

Mio caro, buon Professore,

Finalmente, dopo naturali peripezie, per il valido, fraterno aiuto di Carlos Cossio, sono stato contrattato per la cattedra d'Introducción al Derecho e per un corso di conferenze d'Introduzione alla Filosofia nella facoltà di diritto di Tucumán.

professor Oscar Sarlo, si tratta di Juan Llambías de Acevedo, uno dei maggiori filosofi uruguaiani di quegli anni e profondamente interessato alla cultura tedesca.

Treves (1939). 
Desidero molto annunciarle la notizia ricordando con quanta affettuosa bontà ha voluto seguirmi e incoraggiarmi nelle mie "peripezie».

Ho iniziato le lezioni da pochi giorni e faccio del mio meglio perché riescano proficue ai numerosissimi studenti che ascoltano, con perfetta serietà, i miei, purtroppo, frequenti strafalcioni di castigliano. [...]

Mi ricordi cordialmente a tutti i miei colleghi cui penso spesso con affettuosa gratitudine. A lei, l'espressione della mia devota amicizia.

Renato Treves ${ }^{44}$

«Quando vidi Couture per la prima volta nell'ottobre del 1938 a Montevideo», ricorda Treves, «ebbi l'impressione di trovarmi di fronte [...] ad una personalità completa e integra e con lui, in successivi incontri, in lunghe gite e in amichevoli conversazioni cominciai a conoscere e ad amare la sua comarca: la Repubblica dell'Uruguay». ${ }^{45}$

Il loro affiatamento derivava anche dal comune atteggiamento contrario a ogni dogmatismo nella scienza e ad ogni dittatura in politica. In particolare, a Couture era estraneo il formalismo giuridico, cui preferiva una concezione vitale e culturale del diritto (ispirata al filosofo uruguaiano Carlos Vaz Ferreira, 1872-1958). Per questo gli interessava anche la sociologia e notava con piacere che non pochi esuli europei scoprivano questo approccio al loro contatto con la cultura sudamericana. ${ }^{\mathbf{4 6}}$

Del suo primo viaggio in Italia, nel 1949, Couture ha lasciato un diario dattiloscritto e illustrato con varie fotografie. In quel viaggio consolidò i suoi contatti con i democratici italiani e Calamandrei ricorda che la malattia mortale colpì Couture nell'aprile del 1956 mentre preparava il discorso per commemorare l'undicesimo anniversario della Liberazione italiana, il 25 aprile, all'Università di Montevideo: discorso che avrebbe dovuto essere intitolato L'università italiana nella Resistenza.

Questa apertura alla democrazia non era però astratta. «Senza posa, ricorda ancora Treves, - Couture si prodigò per accogliere, consigliare e sistemare al di là dell'Atlantico gli intellettuali europei dei più diversi paesi d'origine, spagnoli, italiani, tedeschi, francesi». ${ }^{47}$ Ancora nell'autunno del 1940 gli riuscì di trarre in salvo il processualista tedesco James Goldschmidt.

44 Renato Treves a Eduardo J[uan] Couture, Tucumán, 21 maggio 1939, 2 facciate ms.; lettera conservata nell'archivio privato della famiglia Couture, Montevideo.

45 Treves (1957) 468.

46 Couture (1947) 23; cit. da Treves (1957) 473.

47 Treves (1957) 470. 
E poi, finita la guerra in Europa e sopravvenuto il peronismo in Argentina, continuò la sua opera con gli esuli che lasciavano l'altra sponda del Río de la Plata.

La disponibilità di Couture fu dunque totale, e di questo gli rendeva merito un Calamandrei che - alla fine del 1938, pur attraversando un comprensibile «periodo di grande tristezza» - non aveva perso la speranza in un futuro migliore.

Illustre e caro collega,

mi permetta prima di tutto di esprimerLe la mia commossa riconoscenza per la premura e la comprensione colla quale Ella ha risposto alle mie ultime lettere, e per lo spirito di solidarietà umana con cui Ella ha preso a cuore le mie raccomandazioni. In un periodo di grande tristezza, le Sue lettere mi sono giunte come un motivo per non perdere le speranze nell'avvenire: se, dall'uno all'altro continente, senza precedenti legami di conoscenza personale, basta l'amore degli studi comuni a far sorgere nel momento del dolore le amicizie operose, possiamo continuare con fede il nostro lavoro di giuristi, e sperare ancora che il diritto possa servire prima o poi a riconciliare i popoli della Terra.

Dei due colleghi di cui Ella mi chiede non posso, nel momento, darle notizie recentissime. Ma io credo che la sistemazione sia più urgente, ora, per il L., ${ }^{48}$ e credo che Ella farebbe bene a concentrare su lui le Sue cure. Se avessi notizie più precise mi affretterei a scriverLe.

Sono sempre in attesa delle notizie che Lei mi promise per la «Rivista», ${ }^{49}$ sul movimento della scienza e della legislazione processuale nell'America latina: intanto, nel primo numero del 1939 , dedicherò un capitolo della mia rassegna alla bella rivista diretta da Lei.

La prego di leggere in questa mia lettera assai più cordialità e gratitudine di quanta non Le esprimo: e gradisca, con i migliori auguri per il 1939, una affettuosa stretta di mano dal Suo aff.mo

Piero Calamandrei ${ }^{50}$

Dunque le dittature, le leggi razziali, la guerra alle porte non riuscivano a far tacere le ragioni scientifiche: alla fine, è sempre ad esse che il discorso ritornava. Tuttavia vi tornava non certo per un'incorreggibile deformazione professionale, ma perché solo così quelle generazioni potevano scrivere il loro collettivo De consolatione philosophiae.

48 Calamandrei si riferisce con ogni probabilità a Tullio Liebman.

49 Si tratta della «Rivista di diritto processuale civile», che dopo il 1948 si chiamò soltanto «Rivista di diritto processuale».

50 Piero Calamandrei a Eduardo J. Couture, Firenze, 24 dicembre 1938, 2 pp. ms.; lettera conservata nell'archivio privato della famiglia Couture, Montevideo. 


\section{Dall'Uruguay all'Europa: «quasi in pellegrinaggio»}

Anche la lettera di Calamandrei riportata nel prossimo paragrafo è conservata nell'archivio di Couture a Montevideo. Couture era un corrispondente accurato, quindi in Italia si incontrano altre importanti testimonianze della sua attività culturale, come ad esempio il carteggio con Giorgio Del Vecchio: un carteggio esemplare fra due studiosi accomunati dall'interesse scientifico, cui è dedicato il $\$ 7$. Quando gli esuli europei cercavano rifugio in Sudamerica, il piccolo e democratico Uruguay poteva accoglierli all'arrivo, ma non sistemarli tutti, e perciò Couture operò instancabilmente per collocare i colleghi europei anche in altri Stati: come si è detto, indirizzò James Goldschmidt e Renato Treves in Argentina, oppure Enrico Tullio Liebman e Tullio Ascarelli in Brasile.

Come molti studiosi sudamericani, Couture sentiva che un suo viaggio in Europa sarebbe stato l'indispensabile coronamento della propria cultura. Tuttavia questo sogno venne infranto nel 1931 dalla svalutazione del peso uruguaiano e, nel 1939, dallo scoppio della guerra. Terminato il conflitto, nel 1949 Couture visitò finalmente anche l'Italia: «Consideré siempre este viaje como una necesidad espiritual. Su ausencia era como un hueco en mi cultura. Durante veinte años había hecho el profesor de una civilización que solo conocía de libros, de fotografías, de reproducciones. Nunca mis ojos habían visto un documento importante de esa civilización. Lo que había visto en los Museos de Nueva York no contaba como tal. El año 1949, sería, pues, el año fundamental de mi cultura». ${ }^{\mathbf{5 1}}$

Nel viaggio prendono corpo le persone e gli amici conosciuti soltanto attraverso le lettere. E naturalmente l'incontro più emozionante, ma non privo di autoironia, è quello con Calamandrei:

«iQue momento! Calamandrei es el más fino de todos los juristas italianos que he leído y el mejor escritor. Aquella idea de la balanza con la rosa y los libros, a la que más de una vez he acudido, es suya. Me lo imaginaba viejo, pálido, elegantísimo nel vestir, come es elegantísimo en el pensar y en el escrivir; suave y cortesano en los

51 Album, p. 3. I ricordi del viaggio in Europa sono contenuti in un Album (118 pp. $+1 \mathrm{nn}$.) di grande formato, in cui si alternano fotografie, ritagli di giornale e testi dattiloscritti quasi sempre di piú pagine pinzate insieme per formare un fascicolo. Sono incollate anche lettere altrui, spesso piú d'una nella stessa pagina. Quindi le pagine complessive dell'Album sono ben piú delle 118 numerate: queste ultime, infatti, costituiscono spesso soltanto il supporto per gruppi di altre pagine. D'ora in poi citato come Album. 
modales. Ha sido conmigo de una generosidad extrema. A el debo la Accademia dei Lincei y páginas memorables en la «Rivista di Diritto Processuale».

$¿$ Que me encuentro? A Cravotto.

Tiene la belleza y la eleganza tan problemática de Cravotto; habla como florentino e pronuncia la $c$ como $j$ o como $l$, y por dentro la misma exquisita composición espiritual, política (acaba de votar en la Cámara contra el Pacto Atlántico) y moral de Cravotto. Desilusión física y profunda alegría espiritual». ${ }^{52}$

In Couture la visione del paesaggio italiano è sempre legata a reminiscenze culturali. A Viareggio, rivede «mi viejo y querido amigo: el mar», ma è un mare diverso dall'Oceano Atlantico di casa sua; è il mare del mondo classico, «es agua casi sagrada por tantos siglos de historia humana». ${ }^{53}$ Invece, in auto verso Siena con Calamandrei, prevale la passione giuridica: «Nosotros hablamos de procedimiento; pero la señora nos reprende porque no miramos la montaña». ${ }^{54}$ Della casa di Calamandrei a Poveromo Couture ci lascia un ritratto che fonde l'attenzione per l'babitat dell'amico con il ricordo della storia recente, il tutto pervaso da echi culturali, la cui emozione viene corretta da una finale nota ironica:

«Poveromo. Calamandrei tiene en este lugar, asolado por la artillería de la guerra (la línea gótica se hallava a un kilómetro [...]) una hermosa propriedad en un pinar. Fué ocupada por los alemanes y atacada por las bombas. Hubo que rehacerla casi de nuevo. Ahora están rehaciendo el pinar. Deberán esperar unos 150 años para tenerlo como antes. Adentro, una colección estupenda de Chirico con el apocalipsis. Arriba un mirador en el cual Calamandrei trabaja entre el mar y las montañas imponentes. En ellas nació Carducci, fué Gobernador Ariosto y de ellas (porque allí están las canteras de mármol de Carrara, cuyo mármol se ve áun hasta la cumbre) extrajeron pedazos de piedra Miguel Angel, Donatello y los marmolistas de cementerio de la calle Yaguarón». ${ }^{55}$

I paralleli ironici con Montevideo ricorrono spesso, come ad esempio a Venezia: «Me tomo un vapor y voy al Lido a conocer una de las más famosas playas del mundo. Cuando la arreglen bien, empezará a parecerse a Carrasco». ${ }^{56}$ Complessivamente l'Italia appena uscita dalla guerra lo sorprende. Ormai alla fine del viaggio, a Roma, visita le Terme di Diocleziano con il prof. Tito Ravà e si guarda attorno con stupore: «Frente al lujo que circula

52 Album, p. 2 del fasc. a p. 74.

53 Album, p. 88, fasc. di 7 pp., (sabato 30), p. 1 s.

54 Album, p. 88, fasc. di 7 pp., (sabato 30), p. 1.

55 Album, p. 88, fasc. di 7 pp., (sabato 30), p. 2.

56 Album, p. 2 del fasc. a p. 94. 
por la calle, a la belleza de las mujeres y a su elegancia, muy superiores a todo cuanto he visto en Europa, me pregunto: ¿Y este pueblo perdió la guerra? ¡Qué sería si la hubiera ganado!». 57

La lettera riprodotta nel prossimo paragrafo venne scritta da Calamandrei quando il viaggio di Couture era ormai giunto a termine, e quindi fa riferimento ad avvenimenti legati non soltanto alle giornate fiorentine.

In un'Europa ancora ferita dalla guerra, la prebellica amicizia epistolare con Calamandrei si andava trasformando in una fraterna vicinanza. Ma quelle fitte righe di Calamandrei descrivono, oltre al dischiudersi di un intenso rapporto personale, anche l'atmosfera di quegli anni difficili in cui nasceva la nostra gracile repubblica: il parlamento in subbuglio, la conferenza di Couture alla «Sapienza» nel mezzo degli scontri fra studenti e polizia (episodio ricostruito nel $\ 6$ ), il manifesto con la Venere di Botticelli (annuncio delle manifestazioni lorenziane) bloccato dal Questore di Roma perché ritenuto osceno. Infine, quelle pagine sono anche un richiamo utile per ricordare agli smemorati di oggi com'eravamo più di mezzo secolo fa. Non spero che questa lettera possa far ravvedere qualche revisionista, ma è certamente un frammento vivo di memoria da mettere a disposizione dei più giovani: vedano un po' com'era la destra nei fatti.

\section{$5 \quad$ La lunga lettera di Calamandrei:}

«quasi continuando un discorso iniziato da tempo»

Il viaggio in Europa fu per Couture un'esperienza di eccezionale intensità intellettuale. Anni dopo si riferirà a quel viaggio chiamandolo semplicemente «el inolvidable». Raccolse i suoi ricordi, le foto e le cartoline in un albo, tra le cui pagine si trova incollata anche la lunga lettera che Calamandrei gli scrisse a mano con la data "Marina di Poveromo, Ronchi (Massa Apuana), 6. VIII. 1949», annotando sul margine superiore: «Scusa la lettera manoscritta, ma in questo studio è vietato l'ingresso alle dattilografe!». Poveromo era infatti il buen retiro in cui lavorare indisturbato; e nella quiete di quella casa i ricordi fluivano con serenità dalla penna del giurista fiorentino.

57 Album, p. 114, fasc. di 7 pp., domingo 15 maggio 1949 (ultimo giorno), p. 2. 
Carissimo prof. Couture,

Finalmente mi è riuscito di scappare da Roma e di prendere un po' di riposo in questo rifugio marino: e per prima cosa, da questo studio «aereo» che anch'Ella conosce $^{58} \mathrm{mi}$ metto a scrivere senza la solita malaugurata fretta all'amico Couture: all'amico Couture al quale, con tutta la buona volontà di prestare ossequio al cerimoniale, non mi riesce proprio di continuare a dare del «Lei» e mi vien fatto di dare del «tu» come si fa coi vecchi amici. Caro Couture, io sono, ahimè, più vecchio di te: ed è il privilegio dell'età che mi dà il diritto di prendere questa iniziativa e di munirla subito della clausola di provvisoria esecuzione (a proposito: in quell'incidente in tema di provvisoria esecuzione che mi sentisti discutere, $\mathrm{i}$ giudici mi hanno dato torto: Amen $!^{59}$ ) scrivendoti in quello stesso linguaggio con cui ci si rivolge agli amici d'infanzia.

In verità, in quei troppo pochi giorni che abbiamo passati insieme, io ho avuto l'impressione di trovarmi non con il cittadino di un altro Continente, conosciuto per la prima volta in età matura, e accessibile a me soltanto per la comunanza degli studi, ma con un conterraneo nato e cresciuto nella stessa patria (che è poi questa bumanitas, patria spirituale in cui si riconoscono fratelli tutti gli uomini civili), col quale, fin dal primo nostro incontro, ci veniva naturale di trovarci d'accordo sul modo di concepire il mondo e la vita, e di conversare senza riserve e senza sospetti, sicuri della consonanza, quasi continuando un discorso già iniziato da molto tempo ...

Dunque, caro Couture, io ho da ringraziarti di molti doni (e mi dispiace di non averlo fatto subito dopo la tua partenza, ma proprio non m'è stato possibile: figurati che una volta t'avevo cominciato a scrivere dalla Camera dei Deputati, durante una seduta, ma poi fui costretto a interrompermi, perché sulla mia testa passavano, come nuvole di freccie, le invettive scambiate fra i banchi dei democristiani e quelli dei comunisti ...).

E per prima cosa ho da ringraziarti, in generale, di essere venuto in Italia e di avermi dato modo di rivedere con te qualche panorama naturale e qualche architettura o statua di questo paese nel quale purtroppo la vita mi condanna a vivere tutto concentrato nel mio lavoro frettoloso, senza accorgermi delle bellezze alle quali, chi vive in mezzo ad esse, passa accanto senza avvedersene. Nei giorni che abbiamo

58 Proprio a questo studio alludeva Couture quando, nel suo diario di viaggio citato nel paragrafo precedente, descriveva la casa di Marina di Poveromo: «Arriba un mirador en el cual Calamandrei trabaja entre el mar y las montañas imponentes» (Album, p. 88; qui sono incollate 7 pagine dattiloscritte e il testo qui citato si trova a p. 2 con la data sabato $30)$.

59 Venerdì 29 maggio 1949 Couture aveva annotato nel suo diario di viaggio quanto l'avesse impressionato l'arringa di Calamandrei: «Calamandrei abogado. Voi a la Corte de Apelación donde Calamandrei «discute una causa. En realidad no discute nada. Simplemente aplasta al desgraciado abogado contrario con una exposición de tal orden, claridad y eficacia, que la Corte (que hasta ahora ha escuchado de mal humor todo cuanto se ha dicho, incluso a sus mismos miembros) se queda embelesada» (Album, p. 80; qui sono incollate sei pagine dattiloscritte e il testo qui citato si trova a p. 6 con la data Viernes 29). 
passato insieme in Toscana, eri tu che facevi da guida a me, e non viceversa! Cos̀̀ la tua compagnia è stata una parentesi di arte e di poesia in mezzo alla quotidiana prosa: e questa tua compagnia di uomo intelligente e sensibile, capace di vedere e di distinguere, è stato il più bel dono che mi hai portato dall'Uruguay.

Poi ho da ringraziarti di doni numerosi, più specifici e circostanziati: del telegramma che mi facesti da Roma prima di partire, al quale io risposi telegraficamente all'albergo la sera stessa, ma il mio telegramma mi tornò respinto perché giunto quando eri già partito; della fotografia che rappresenta due vecchi fedeli coniugi sulla scala di una villa; di un programma della Associazione culturale italo-uruguayana, dal quale si apprende che il prof. Couture il 14 di luglio tenne in Montevideo una conferenza su un argomento scelto dalla sua benevolenza di amico (della quale l'amico gli è profondamente grato), la quale conferenza penso che abbia interessato il pubblico unicamente per l'arte dell'oratore, la quale arte è capace di trasformare magicamente in bellezza anche i soggetti più umili ... ${ }^{\mathbf{6 0}}$

E finalmente ho da ringraziarti del dono, giuntomi due giorni fa, de Los Mandamientos del Abogado, ${ }^{\mathbf{6 1}}$ che mi accingo a leggere e a recensire in questo periodo, proprio mentre sto lavorando alla terza edizione dell'Elogio dei giudici. ${ }^{\mathbf{6}}$ Ben volentieri scriverò, se lo desideri, una breve introduzione al tuo libretto, al quale auguro, ed è prevedibile che avrà, la meritata fortuna.

Nel numero di luglio del Ponte, che a quest'ora dovrebbe già esserti arrivato, vedrai che sei ricordato due volte: ‘Calamandrei continua a scrivere sul bordo sinistro della lettera $>$ la prima per quell'incidente studentesco al quale ti trovasti testimone all'Università di Roma; ${ }^{63}$ la seconda per la pittura di Pedro Figari, ${ }^{64}$ della quale io

60 Nel settembre 1949 la Asociación cultural italo-uruguaya aveva organizzato a Montevideo una mostra del libro italiano. Il quotidiano di Montevideo «El País» del 7 settembre 1949, nel darne l'annuncio, pubblicò una fotografia degli organizzatori, fra cui è presente anche Couture. Il 14 settembre lo stesso quotidiano annunciava la conferenza di Couture intitolata Piero Calamandrei, un jurista italiano que sabe algo más que derecho: a questa conferenza si riferisce il testo di Calamandrei. Devo queste informazioni alla cortesia dell'amico Oscar Sarlo, dell'Universidad de la República di Montevideo.

61 Couture (1949). Nella Biblioteca Giorgio Del Vecchio dell'Università «La Sapienza» di Roma sono conservati due esemplari di quest'opera, uno dei quali porta una dedica autografa: "Al Prof. Giorgio del Vecchio, Mensaje de cordial afecto de C.». Una recensione di Renato Treves a questo libro di Couture si trova in «Rivista Internazionale di Filosofia del Diritto", 1951, 243-244.

62 La revisione e l'ampliamento di quest'opera - Calamandrei (1954) - doveva durare ancora alcuni anni. La terza edizione risulta più che raddoppiata rispetto alla prima (1935, 145 pp.) e alla seconda (1938, XXIV, 171 pp.). Ne esiste anche una traduzione spagnola: Góngora, Madrid 1936.

63 Cfr. infra, $\$ 6$; Calamandrei si riferisce agli scontri studenteschi avvenuti sabato 14 maggio 1949 nell'Università di Roma, proprio quando Couture doveva tenere la sua conferenza alla Facoltà di Giurisprudenza.

64 Calamandrei (1949). Il pittore Pedro Figari (1861-1939) suscitò un vivo interesse in Calamandrei, tuttavia a Firenze non ho trovato documenti sull'effettiva realizzazione della mostra auspicata in questa lettera. 
vorrei che si potesse tenere a Firenze una mostra nella prossima primavera. Sarà possibile?

Vedrai che in quella nota che ho scritto sul Ponte a proposito del Figari si allude in un punto alla Venere di Botticelli. ${ }^{65}$ L'allusione, per te altrimenti incomprensibile, si riferisce a questo episodio: pochissimi giorni dopo che tu eri partito, la polizia di Roma vietò l'affissione di un manifesto della mostra fiorentina di Lorenzo sul bordo destro il Magnifico perché vi era riprodotta la immagine della Venere del Botticelli, considerata, dalla polizia democristiana, oscena e contra bonos mores ... Allora io presentai al Ministro una interpellanza ironica, chiedendo che il quadro originale sia tolto dalla Galleria di Firenze, per non offendere il pudore del pubblico (te compreso). E allora il Ministro, temendo il ridicolo, revocò l'assurdo divieto! ...

Arrivederci, caro Couture, scrivimi a lungo: ed anche a nome di mia moglie porgi i più cordiali ossequii alla Tua gentile Signora e alle tue bambine, che ci sembrano già persone di vecchia conoscenza ... Un abbraccio dal tuo

Piero Calamandrei

Proporre oggi questa lettera vuol dire anche ricostruire qualche piccola tessera nel mosaico del dopoguerra italiano; e allora vale la pena ritornare sugli incidenti all'università di Roma, non soltanto per comprendere il contesto in cui si muoveva Couture, ma anche per ricordare come stava nascendo quella repubblica italiana che oggi si tende a vituperare, dimenticando quante libertà ci vengono dalla sua Costituzione, allora appena approvata e anch'essa oggi vituperata.

\section{Couture e gli incidenti alla «Sapienza» del 14 maggio 1949}

Cercherò di far parlare i giornali, oggi in parte scomparsi o trasformati. Già le testate lanciano un messaggio: nel 1949 la voce della destra, «Il Secolo d'Italia», non esisteva ancora (nascerà infatti nel 1952, come uno dei tanti frutti della guerra fredda, e dal 2012 sarà soltanto on line), mentre «L'Unità», voce della sinistra allora così forte ed estrema, dapprima ha taciuto (nel 2000), poi ha ripreso flebile.

Forse può sembrare che i giornali di allora avessero riservato poco spazio alla notizia degli scontri all'università romana, ma oggi - abituati ad affron-

65 Il passo cui si riferisce Calamandrei è contenuto in una parentesi aperta nel discorso sulla visita di Couture: «Ho avuto la gioia di fargli io stesso da guida per le strade e le gallerie di Firenze, riscoprendo con lui questi miracoli, commovendomi della sua commozione e lasciandolo estasiato e rapito dinanzi alla Venere del Botticelli: senza rivelargli, per carità di patria, che i nostri paterni governanti ... beh, lasciamo correre!» (Calamandrei [1949] 903). 
tare quotidiani di oltre una cinquantina di pagine - ci è difficile immaginare che cosa fossero i giornali del dopoguerra: a metà maggio 1949, per esempio, in sole quattro pagine i giornali dovevano informare su un mondo in fermento - Shanghai stava cadendo in mano all'esercito di Mao Tse Tung, i tedeschi dell'Est eleggevano il loro nuovo governo su una lista unica, i socialisti italiani si riunivano in un congresso su cui incombeva la minaccia della scissione - e sulla cronaca nazionale e locale, lasciando anche spazio alla pubblicità e ai piccoli annunci economici. Il lunedì, poi, niente giornali; e negli altri giorni quattro pagine a quindici lire, perché la carta era razionata.

Il sabato 14 maggio 1949 Couture, che si era recato alla «Sapienza» per tenervi una conferenza, venne coinvolto negli scontri fra studenti di opposte affiliazioni politiche. I giornali diedero la notizia del tumulto nel numero della domenica, che, per «L'Unità», era un numero destinato ad essere diffuso con lo «strillonaggio» volontario degli iscritti al Partito Comunista. «L'Unità» è il giornale che dà maggior rilievo alla notizia dei fatti di Roma, riportandola in prima pagina con un breve articolo su due colonne: «Violenze fasciste all'Università di Roma. - I gravi incidenti si sono verificati proprio mentre Scelba proibiva il congresso dei giovani democratici». ${ }^{\mathbf{6 6}}$ Quest'ultimo sottotitolo esige una breve spiegazione. Proprio in quei giorni il Consiglio dei Ministri, di cui Mario Scelba faceva parte come Ministro degli Interni, aveva proibito una manifestazione per la pace - il tema della «pace» era allora appannaggio delle sinistre - che avrebbe portato a Roma centomila giovani da ogni parte d'Italia. Il divieto, su cui ritornano anche le ultime righe dell'articolo citato, era così formulato: «Allo scopo di evitare possibili turbamenti dell'ordine pubblico nella Capitale [il Consiglio dei Ministri] ha deliberato che tali concentrazioni non siano autorizzate». ${ }^{67}$

Il cronista dell'«Unità» non praticava certo una weberiana astinenza dai giudizi di valore, ma è dal suo articolo (debitamente sfrondato) che oggi rispetto alle altre fonti giornalistiche che ho potuto vedere - si può desumere la maggior quantità di informazioni su fatti e antefatti. «L'Università di Roma, - queste le parole con cui si apre il resoconto de «L'Unità», - è stata ancora oggi teatro di una vergognosa aggressione fascista contro gli studenti.

66 «L'Unità. Organo del Partito Comunista Italiano», 15 maggio 1949, p. 1; poiché quella che viene qui citata è l'edizione dell'Italia settentrionale, non è escluso che notizie più dettagliate siano contenute nell'edizione romana.

«L'Unità», 14 maggio 1949, p. 1. 
Già da alcuni giorni i giovani fascisti del M.S.I. avevano cercato di provocare incidenti durante le votazioni per il Consiglio interfacoltà, votazioni che si svolgono in seguito all'invalidazione delle precedenti elezioni nelle quali il M.S.I., in virtù di una serie di palesi imbrogli, aveva avuto la maggioranza». ${ }^{68}$

La descrizione del tumulto in cui venne a trovarsi Couture è precisa e, per così dire, tecnica, nel senso cioè di esser stilata da qualcuno che, di quel tipo scontri, doveva avere esperienza diretta: «Questa mattina una settantina di fascisti reclutati nei loro ritrovi abituali, i caffe della Capitale, facevano irruzione nell'aula della Facoltà di Legge nel tentativo di rovesciare le urne di votazione. Pochissimi erano tra loro gli universitari. I fascisti erano prontamente rintuzzati dagli universitari presenti nell'aula, ma ben presto, essendo armati di bastoni, spranghe di ferro, pugni di ferro, e dopo aver lanciato contro gli studenti bottiglie di ammoniaca, avevano il sopravvento. Gli aggressori iniziavano poi una vera battuta per i viali dell'Università, colpendo gli studenti isolati e assalendo chiunque si trovasse nei paraggi della Facoltà di Legge. Una quindicina di studenti risultavano feriti e molti dovevano essere ricoverati all'ospedale».

«L'Unità» inquadrava infine quegli scontri nel più vasto clima di tensione politica di quei giorni: «L'indignazione nell'università è fortissima. Conosciuta l'aggressione, circa quattrocento studenti si radunavano nel piazzale dell'Università per protestare. Non si mancava di rilevare ovunque che l'aggressione fascista è avvenuta proprio nel momento in cui Scelba annunciava limitazioni al diritto di organizzazione delle forze democratiche studentesche», quando cioè veniva vietata quella manifestazione per la pace cui si accennava poco sopra.

Anche il «Corriere della Sera», pur occupandosi prevalentemente di notizie dell'Italia settentrionale, pubblicò nelle pagine interne un breve trafiletto, in cui menzionava anche l'involontario coinvolgimento degli uruguaiani. Il titolo è uguale a quello dell' «Unità», salvo che vi si parla di «missini» ${ }^{69}$ invece che di «fascisti»: «Violenze di 〈missini〉 all’Università di Roma. - Una ventina di feriti - L'ambasciatore dell'Uruguay uscito illeso dalla zuffa». La sintetica descrizione del quotidiano milanese tiene conto del fatto che i suoi lettori non sono romani e che quindi non conoscono gli antecedenti dell'evento:

68 «L'Unità, 15 maggio 1949, p. 1.

69 Con questo nome si indicavano gli aderente al Movimento Sociale Italiano (MSI), il partito erede del Partito Nazionale Fascista. 
«Un'ennesima zuffa, più violenta delle altre, si è accesa stamane all'Università, dove, come è noto, si stanno svolgendo le elezioni suppletive per il consiglio dell'Interfacoltà. Dopo gli incidenti dei giorni scorsi, causati secondo un comunicato emesso ieri dai rappresentanti di tutti i partiti da elementi neo-fascisti, stamane decine di goliardi si erano schierati davanti alle urne onde permettere che la votazione avvenisse liberamente, e per proteggere le urne stesse che ieri erano state fracassate. Improvvisamente, un gruppo di giovani penetrato nell'ateneo tentava di impadronirsi delle urne: ne nasceva una colluttazione, nella quale rimaneva coinvolto anche l'ambasciatore dell'Uruguay, che si stava recando ad ascoltare una conferenza [quella di Couture] ma che si è trovato circondato da un mare di giovani urlanti. Fortunatamente, però, egli è uscito illeso dalla zuffa. Persino delle barricate venivano innalzate con i banchi e con le cattedre. La battaglia si è protratta per un'ora circa, finché, avvertita dagli stessi studenti, è giunta la ¿Celere ${ }^{70}$ I feriti e i contusi ammontano a una ventina». ${ }^{\mathbf{7 1}}$

La descrizione dei fatti coincide dunque tanto sul foglio della borghesia milanese, quanto sull'organo del maggior partito comunista dell'Occidente. Anzi, le ultime righe del «Corriere» trasmettono una visione più corale e più democratica della reazione a quell'aggressione: «I rappresentanti dei partiti democristiano, comunista, socialista, repubblicano, liberale e monarchico hanno diramato un altro comunicato in cui ribadiscono le accuse contro i «missini), affermando, tra l'altro, che sgruppi di facinorosi, formati da elementi non universitari, armati di manganelli, hanno fatto irruzione nella sala ove si svolgevano le elezioni, guidati da studenti di nome, ma non di fatto». Da parte degli studenti afferenti al M.S.I. le accuse vengono ribattute».

Infine, nel diario di viaggio di Couture è incollato un ritaglio del «Messaggero» di Roma, che annunciava nei titoli di testa: «Movimentate elezioni allo Studium Urbis - Per la prima volta la polizia entra nell'Università per sedare i violentissimi incidenti accaduti ieri mattina. - Facinorosi irrompono nell'aula delle votazioni. Il professore uruguaiano Couture che doveva tenere una conferenza sorpreso dagli incidenti. Numerosi feriti». ${ }^{72}$ Il quotidiano

70 I «Reparti celeri» della Polizia di Stato repubblicana svolgevano anche le funzioni dei corpi che altrove venivano chiamati «antisommossa».

71 «Il Corriere della Sera», 15 maggio 1949, p. 4.

72 Album, p. 112, cui è incollato un fascicolo di 5 pp. dattiloscritte e un ritaglio del «Messaggero» di domenica 15 maggio 1949. 
romano descrive l'irruzione nella sala delle votazioni e anche il salto dalla finestra d'un responsabile di seggio, per portare in salvo l'urna elettorale. E si sofferma anche su quanto stava capitando agli uruguaiani convenuti per ascoltare Couture: «Uno spiacevolissimo episodio che gli studenti stessi hanno deplorato, - informa «Il Messaggero», - è accaduto quando è giunto il professore uruguaiano Couture che doveva tenere una conferenza proprio nell'aula di Giurisprudenza. Il professor Couture era accompagnato dal Ministro dell'Uruguay e dal Rettore Magnifico. Mentre le personalità ascendevano la scalea di accesso all'aula sono esplosi gli incidenti più gravi, tanto che l'auto del Ministro, per ordine del Diplomatico stesso, ha trasportato al vicino Policlinico i feriti».

La cultura degli anni fascisti impregna ancora la lingua dei giornali democratici della borghesia: quelli che per «L'Unità» sono «bastoni» e «universitari», nel «Corriere della Sera» divengono «manganelli» e «goliardi», mentre nel «Messaggero» gli uruguaiani non salivano la scalinata antistante la facoltà, ma «ascendevano la scalea».

Fin qui i fatti. Un commento politico illuminante sulla tensione ideologica di quei momenti viene dalla rubrica Ritrovo (in cui mensilmente veniva commentata l'attualità) del «Ponte» del luglio 1949. Diversamente dal solito, Enzo Enriques Agnoletti ${ }^{73}$ apre quella rubrica con un corsivo senza titolo, che è uno smarrito commento alla Cronaca dei fatti universita$r i{ }^{74}$ pubblicata nelle pagine immediatamente successive. Commento smarrito, perché quella Cronaca riflette una vicinanza ideologica fra estrema sinistra ed estrema destra che a Enriques sembra quasi un ritorno di tempi weimariani.

Anche se non sono riuscito ad accertarne l'identità, l'autore di quella Cronaca è uno degli studenti feriti trasportato all'ospedale con l'automobile dell'ambasciatore uruguaiano. È uno studente della sinistra rivoluzionaria, un fautore dell'«intransigenza operaia» contrario alla «nefasta politica di unità nazionale» del Partito Comunista Italiano. Nell'auto ne discute con un dirigente comunista proveniente dal Partito d'Azione, poi, all'ospedale,

73 Enzo Enriques Agnoletti (1909-1986) si laureò con Calamandrei, di cui divenne poi assistente. Fu co-fondatore del «Ponte» e poi suo direttore dal 1956, alla morte di Calamandrei. In politica fu dapprima azionista, poi socialista finché Craxi lo espulse dal Partito Socialista Italiano (Dizionario biografico degli italiani, vol. 42, 795-797).

74 L. I., Cronaca dei fatti universitari, «Il Ponte», V, luglio 1949, n. 7, 892-895. Non sono riuscito ad accertare chi sia L.I. Da queste pagine sono tratte le citazioni che seguono. 
«mentre mi disinfettano, sento uno che mi chiama: è Giorgio Ciarrocca, noto dirigente neofascista, che avevo conosciuto nel '42 quando egli, corporativista di sinistra, aveva avuto amichevoli discussioni con me e con il mio amico Massimo Gizzio, ucciso poi dai fascisti nel '44. Ci mettiamo a discutere mentre ci fanno le cure del caso». Gli scontri, come si è detto, avvennero il sabato 14 maggio; lunedì 16 anche questo studente viene accompagnato all'Ufficio Politico della Questura, dove incontra alcuni neofascisti: con essi ha «un'interessante discussione» che rivela i dilemmi ideologici di tutti loro. «Musillo dichiara, - continua lo studente della sinistra rivoluzionaria, - che i giovani del MSI non vogliono più i gerarchi del ventennio, che hanno tradito le aspirazioni rivoluzionarie del fascismo, e mi parla con aperto disprezzo del regime di Franco, che egli definisce «non fascista, ma democristianos. Parliamo anche delle interpretazioni «eretiche> del Risorgimento; di Cattaneo, di Gobetti, di Dorso. A proposito delle correnti di opposizione nel fascismo, il mio interlocutore esprime il sospetto che l'incidente in cui morì Padovani sia stato provocato dai reazionari del fascismo» (cioè, per capirci, dai fascisti di destra). Segue una momentanea detenzione: «Nella cella ci sono due neofascisti giovanissimi, arrestati per aver fischiato il Capo del Governo [...]. Dopo esserci raccontati i nostri reati ci facciamo reciproche congratulazioni». Infine, viene la delusione per il mancato rinnovamento della giovane Repubblica italiana: «Ci portano tutti a Regina Coeli. Sei anni prima, esattamente quel giorno, c'ero stato portato dall'OVRA mussoliniana. Ma allora c'era il fascismo. Adesso invece c'è ... la democrazia! Infatti gli agenti non hanno più i fasci, ma la sigla della repubblica. E non ci sono più sui muri neanche $\mathrm{i}$ cartelli con le frasi carneluttiane che facevano bella mostra di sé ancora nel dicembre del '47. Ci chiudono con dei collaborazionisti slavi, richiesti dall'URSS come criminali di guerra». Questa era, dunque, l'aria dei tempi: aria mossa da fedi non tiepide.

Ancora una volta ritorna la solita e ambigua contiguità del fascismomovimento con la sinistra rivoluzionaria, che esisteva negli anni Venti e che più di recente si ritrovava anche in certe sfuggenti e fuggevoli comunanze di vedute tra il neofascismo di Pino Rauti e certe frange della sinistra extraparlamentare. Certo, per chi come Enriques affrontava la costruzione della nuova repubblica animato dagli ideali rinnovatori della Resistenza, quell'intreccio risultava non solo indigesto, ma incomprensibile: di qui il suo sgomento davanti a quella Cronaca. «Raramente, - commenta, - abbiamo letto un documento che ci abbia fatto più impressione di quello che qui 
riportiamo». Quel mondo giovanile «spaccato in due come una mela» provoca in Enriques un senso di impotenza: «L'impotenza che ogni generazione sente per non riuscire a comunicare le proprie esperienze alle generazioni successive».

Il diario di viaggio di Couture associa nel ricordo l'università di Parigi e quella di Roma: mentre a Parigi, al momento di iniziare la conferenza, si era sentito nella «fossa dei leoni» sotto il peso dei ricordi culturali, Roma fu per lui la discesa nella «gabbia delle tigri sanguinarie». Il parallelismo delle due formulazioni maschera però una marcata divergenza fra la realtà culturale parigina e la realtà politica romana. «Nel momento in cui giungiamo all' $U$ niversità, - registra nel diario di viaggio, - con l'ambasciatore Giambruno [...], una valanga di studenti scende la scala portando un ragazzo con un braccio rotto, un altro con la testa insanguinata, un altro azzoppato; e tutto in mezzo a una gazzarra infernale. Questo era dunque il mio pubblico: sinceramente, lo trovavo un po' rumoroso". Mentre l'ambasciatore metteva la sua auto a disposizione dei feriti, ci si chiedeva se, in quelle condizioni, si sarebbe potuta tenere la conferenza. Infine subentrò un po' di calma, e la conferenza ebbe inizio.

Di fronte al consueto folklore d'ogni contestazione universitaria, il commento politico di Couture è pacato e colmo di spirito democratico non ostentato a parole, ma vissuto con naturalezza. Indubbiamente il tumulto lo aveva sorpreso e preoccupato: però «non tanto quanto gli italiani, aggiunge, - che sono desolati e non sanno che spiegazione fornirmi. Io non do peso all'evento perché, a dire il vero, di tumulti studenteschi ne so qualcosa». Ma per gli italiani è diverso, nota Couture, perché «sono ancora abituati all'ordine spolaccos (la pace di Varsavia fondata sulle baionette) dei bei tempi del fascismo, e non sanno come spiegarselo». ${ }^{75}$ Dunque, la reazione di Couture è quella di chi è abituato alla democrazia, mentre quella

75 Ecco le parole dell'Album di Couture: «No tanto como los italianos, que están desolados y no saben qué explicaciones darme. Yo le quito importancia al asunto, porque la verdad es que de algaradas estudiantiles conozco algo. Ellos que están todavía abituados al orden <polaco> (la paz de Varsovia apoyada en bayonetas) de los buenos tiempos del fascismo no le encuentran explicación». E poi: «Giambruno conduce en su auto a los heridos; se produce desconcierto en el sentido de saber si asistirá público a la conferencia; se arremolina la gente en un mundo de conjecturas y cuando una leve ráfaga de paz se cierne sobre la casa, se dispone que la conferencia comience» (112, fasc. di 5 pp.; ritaglio del «Messaggero», domenica 15 maggio 1949). 
dei suoi colleghi romani è quella di chi sta ancora abituandocisi, e forse non senza qualche difficoltà: è chiaro quindi perché una salda affinità elettiva avesse legato Couture e Calamandrei istintivamente e fin dal primo momento, quando non s'erano mai visti e vivevano separati dall'oceano.

\section{Couture e il filosofo del diritto Giorgio Del Vecchio}

Il panorama dei contatti italiani di Couture richiede anche un cenno alla corrispondenza con Giorgio Del Vecchio (1878-1970), uno dei principali filosofi del diritto italiani del XX secolo. Questo carteggio comprende trentasette documenti, che coprono un arco di diciotto anni: dal 1938 - quando il giovane giurista uruguaiano aveva 34 anni e il già affermato filosofo del diritto italiano sessanta - fino al 1956, anno della morte di Couture. L'ultima sua lettera a Del Vecchio venne scritta poco più d'un mese prima di quell'improvvisa morte. Alla trascrizione e al commento di questo carteggio ho dedicato un articolo, al quale rinvio. ${ }^{76}$

Si tratta d'una corrispondenza in certa misura discontinua, poiché la guerra e le vicende personali provocarono pause e rallentamenti; tuttavia, per altro verso, essa è costante per contenuti e intensità: la si può infatti considerare un carteggio esemplare fra due studiosi accomunati dall'interesse scientifico. Verrebbe da aggiungere: quasi unicamente dall'interesse scientifico, più che da altre comuni passioni, soprattutto ideali o politiche. Da quest'ultimo punto di vista, infatti, il corrispondente ideale di Couture fu Piero Calamandrei.

La corrispondenza con Del Vecchio evita ogni accenno alla politica, poiché in questo campo le posizioni dei due giuristi erano antitetiche. Del Vecchio era direttamente coinvolto nell'attività politica del partito fascista ed aveva aderito con entusiasmo a quel movimento fin dalle sue origini. Invece Couture era profondamente democratico e spesso (ma non nelle lettere a Del Vecchio) formulava giudizi taglienti sulle dittature sudamericane ed europee. Per esempio, visitando il Collegio di Spagna a Bologna, Couture annota: «Lujosísimo, con un director que hace 32 años que está y habla italiano mucho peor que yo, que nos convita con jerez, se hace llamar ¿Su Excelencia〉 y tiene un retrato de Franco que parece un monumento 
equestre sin caballo ... o sin caballero». ${ }^{77}$ Un tacito accordo li portava a parlare di quanto li accomunava e a tacere di quanto li divideva.

Basti qui esaminare la sola lettera di Couture scritta nel 1938 - anno fatale per l'Italia - con cui si apre il carteggio. Da essa si desume come il rapporto fra i due giuristi fosse già iniziato, ma non da molto tempo. Il tono formale che la caratterizza resterà invariato anche nelle lettere successive.

Muy estimado Prof. Del Vecchio:

Con algún retardo he recibido el envío de los opúsculos que ha tenido la exquisita cortesía de enviarme. [...] - Le ruego crea en mi muy especial agradecimiento por tal remisión, que supone una gentil deferencia de un Maestro a quien admiro de largos años atrás. Sus estudios son conocidos y admirados en el Río de la Plata, desde hace años, aun desde el tiempo de nuestros estudios universitarios. La traducción de la «Filosofia del Diritto» ${ }^{78}$ hecha por nuestro común y querido amigo el Prof. Recaséns Siches, ${ }^{79}$ ha tenido la virtud de aumentar el número de lectores en aquellos grupo[s] de estudiosos que han tenido alguna dificultad derivada del idioma. De su actual envío me eran conocidos «Il problema delle fonti del diritto positivo» ${ }^{\mathbf{8 0}}$ $\mathrm{y}$ «La crisi della scienza del diritto ${ }^{\mathbf{8 1}}$ que fueron hace ya algunos [años] traducidos en algunas revistas españolas. [...] - Será para mi un vivo placer publicar una breve reseña de sus opúsculos más recientes ${ }^{82}$ en «La Revista de Derecho, Jurisprudencia y Administración» que dirijo, preocupándome de inmediato de hacer llegar ese comentario a sus manos. [...] - Le ruego, mi muy distinguido Maestro, acepte mis saludos, mi amistad y la seguridad de mi muy viva estima.

E.J. Couture ${ }^{83}$

77 Album, p. 2 del fasc. a p. 100.

78 Couture si riferisce alle edizioni spagnole allora in circolazione: Filosofía del derecho (DEL Veсchio [1930]); oppure alla Filosofía del derecho (Del Vecchio [1935], [1936a]. - Nella biblioteca di Couture ebbi la possibilità di vedere l'edizione italiana di quest'opera, con dedica autografa, ma senza data: Del Vecchio (1936b).

79 Il filosofo del diritto Luis Recaséns Siches, nato in Guatemala nel 1903 e morto in Messico nel 1977, arricchì di ampi commenti la traduzione in spagnolo delle Lezioni di filosofia del diritto di Del Vecchio, fornendo un dettagliato panorama della filosofia del diritto sudamericana.

80 L'articolo è del 1934: Del Vecchio (1934b). Nella biblioteca di Couture sono presenti due estratti di questo scritto. Un fascicoletto porta una dedica autografa: Del Vecchio (1938). Sempre nella biblioteca di Couture è presente anche il estratto di data anteriore, ma senza dedica: Del Vecchio (1936c).

81 Del Veсchio (1934a). Il fascicolo è presente ancor oggi nella biblioteca di Couture.

82 La recensione all'articolo di Del Vecchio (1934b) venne pubblicata nella «Revista de Derecho, Jurisprudencia y Administración» 37, 1939, p. 383.

83 Lettera di Eduardo J. Couture a Giorgio Del Vecchio, Montevideo, 9 dicembre 1938. 
Mentre Couture scriveva questa lettera, le leggi razziali emanate dall'Italia avevano colpito anche Giorgio Del Vecchio. Le «varie circostanze» che gli hanno reso la «vita alquanto difficile», accennate con grande ritegno e quasi di sfuggita nella successiva lettera di Del Vecchio, ${ }^{\mathbf{8 4}}$ sono in realtà eventi sconvolgenti. In forza delle leggi razziali del 1938, Del Vecchio - che pure era stato un fascista della prima ora e che poi era divenuto il rettore fascista (anche se non troppo amato dal regime) dell'Università di Roma - venne allontanato il 25 ottobre di quell'anno dalla carica e dall'insegnamento, in quanto ebreo. Questo spiega perché, nella successiva lettera del 23 febbraio 1940 a Couture, Del Vecchio accenni alla sua attività di direttore della biblioteca presso l'«Istituto internazionale per l'unificazione del diritto privato», a Roma.

Anche l'accenno a Enrico Tullio Liebman e a Renato Treves si ricollega alle leggi razziali, che avevano costretto i due docenti ad abbandonare l'Italia e che, a Montevideo, avevano incontrato Couture.

La sintonia scientifica e la distonia emotiva fra i due giuristi determinano dunque il tono controllato e, a volte, formale del carteggio. Come si è visto, questo non era lo stile abituale di Couture: la sua natura solare lo portava a scrivere agli amici totalmente sintònici lunghe lettere che talora si trasformavano in piccoli capolavori di questo genere letterario. Oltre alle lettere fin qui viste, anche un piccolo campionario di lettere genuinamente couturiane inviate a un amico argentino (e pubblicato poco dopo la morte di Couture $^{85}$ ) può fornire un termine di paragone con cui confrontare il più dimesso carteggio con Del Vecchio: un carteggio fra due professori, non fra due amici.

Il carteggio è conservato nell'Archivio Giorgio Del Vecchio (presso l'Istituto di Filosofia del Diritto della Facoltà di Giurisprudenza dell'Università «La Sapienza» di Roma) e nell'archivio privato della Famiglia Couture a Montevideo. Nell'Istituto di Filosofia del Diritto ora ricordato si trova anche la Biblioteca Giorgio Del Vecchio, in cui sono conservati numerosi scritti di

84 Lettera di Giorgio Del Vecchio a Eduardo J. Couture, Roma, 23 luglio 1939.

85 Bruera (1958). Bruera fu professore di filosofia del diritto nella Universidad Nacional del Litoral di Rosario, in Argentina, e rese pubblici ampi stralci del suo carteggio con Couture commemorandolo il 28 maggio 1957 in una conferenza presso il Colegio de Abogados di Montevideo. 
Couture. ${ }^{86}$ La presenza di tanti scritti processualisti nella biblioteca di un giusfilosofo non deve sorprendere: Giorgio Del Vecchio fu anche un grande avvocato del foro romano.

86 Ecco l'elenco delle pubblicazioni di Couture conservate nella Biblioteca Giorgio Del Vecchio, seguite dalle rispettive segnature:

- Algunas observaciones preliminares sobre la enseñanza del derecho en los Estados Unidos, Faculdad de Derecho, Montevideo 1955, pp. 35-42 [Misc 287/43 (manca); Misc 207/8];

- Espíritu y técnica en el derecho contemporáneo, Antología Jurídica, Buenos Aires 1938, 16 pp. [Misc 449/12 - Misc. 207/3: con dedica autografa];

- El principio de libertad en el sistema del proceso civil (esquema), Antología Jurídica, Buenos Aires 1939, 44 pp. [Misc 206/20];

- El concepto de fe pública, Facultad de Derecho, Montevideo 1947, 101 e 7 pp. [Misc. 206/ 22];

- Oralidad y regla moral en el proceso civil, Antología Jurídica, Buenos Aires 1939, 27 pp. [Misc. 207/11];

- Ciencia y conciencia del derecho, Colegio de Abogados, Montevideo 1954, 7 pp. [Misc. 207/7];

- Concubinado, sociedad de hecho y medidas de seguridad, «Revista de Derecho, Jurisprudencia y Administración», vol. 44, p. 164 [Misc. 207/2];

- El deber de decir la verdad en el juicio civil, «Revista de Derecho, Jurisprudencia y Administración», vol. 36, p. 53 [Misc 207/9];

- Los mandamientos del abogado, Depalma, Buenos Aires 1949, 59 pp. [Misc 207/5: con dedica autografa: cfr. nota 61];

- Introduction à l'étude de la procédure civile, Sirey, Paris 1950, 68 pp. [Misc 207/10];

- La experiencia de los tratados de Montevideo, Montevideo 1949, 31 pp. [Misc. 207/12];

- Medio siglo de derecho, Montevideo 1944, 33 pp. [Misc 207/4];

- Solución política y solución jurisdiccional en los procesos del trabajo, Montevideo 1948, 15 pp. [Misc. 207/1];

- Prólogo, in: Roberto Puig, Los vocablos del derecho, Montevideo 1952, pp. 3-4 [Misc. 216/ 9];

- Prólogo, in: Lino Rodriguez, Arias Bustamante, Concepto y fuentes del derecho civil español, Bosch, Barcelona 1956, pp. I-XIII [30. L. 21];

- La comarca y el mundo, Alfar, Montevideo 1953, 146 pp. [30. D. 10];

- La constitution uruguayenne de 1952, Paris 1953, 118 pp. [30. D. 11];

- Carnelutti y nosotros, in Scritti in onore di Francesco Carnelutti, Cedam, Padova 1950, pp. 315-334 [38. C. 2];

- Protección jurídica de los descubrimientos científicos, in: Jornadas de derecho comparado, Montevideo 1955, pp. 294-298 [39. G. 6]; insieme con El derecho comparado y la comprensión de los pueblos (pp.35-41); Discurso de clausura (pp. 444-446); Neutralidad deljuez (pp. 560-571);

- El proceso como institución, in: Studi in onore di Enrico Redenti, Giuffrè, Milano 1951, pp. 349-373 [38. E. 1];

- Prefazione, in: James Goldschmidt, Problemi generali del diritto, Cedam, Padova 1950, pp. 1-9 [34. L. 29]. 


\section{Comparazione giuridica e storia contemporanea del diritto:}

«scripta volant?»

Le biografie dei giuristi traghettatori di idee fra i continenti e la storia contemporanea del diritto fanno parte di quella ricerca di base (Grundlagenforschung) che fornisce informazioni a più discipline e, quindi, favorisce la ricerca interdisciplinare. In particolare, le biografie dei giuristi e la storia contemporanea del diritto possono essere utili strumenti ausiliari per i comparatisti perché, illuminando il generale contesto culturale in cui avvengono il trasferimento e la recezione di idee e norme giuridiche, possono gettare luce sulle ragioni dell'accettazione o del rifiuto dell'una o dell'altra soluzione giuridica.

Queste biografie a noi vicine sono parte integrante della storia contemporanea del diritto. Essa si rivolge soprattutto al secolo XX ed ha una sua metodologia che, come ho cercato di dimostrare altrove, presenta una sua utilità non solo conoscitiva, ma anche pratica. ${ }^{87}$ Vale dunque la pena di dedicarle attenzione: ma qui cominciano le difficoltà, soprattutto perché gli archivi giuridici contemporanei sono ancora magmatici. Mi limiterò ai soli archivi cartacei, cioè a quelli tradizionali, lasciando da parte quelli relativi alle immagini, ai suoni e all'informatica, che sono non meno importanti, ma che presentano difficoltà tecniche differenti. Lasciamo da parte anche gli archivi degli enti pubblici, che sono (o dovrebbero essere) ordinati e conservati, ma che spesso sono di fatto poco accessibili a causa delle barriere burocratiche. Lasciamo da parte pure gli archivi delle case editrici, strumenti insostituibili per ricostruire segmenti di storia della cultura.

Il momento cruciale per l'archivio personale di un giurista sono i primi decenni dopo la morte del suo titolare, quando i documenti non suscitano ancora la reverenza derivante dall'antichità, ma non presentano più l'utilità pratica derivante dall'attualità. Non sono né antichi né nuovi: sono solo vecchi. È in quel momento che bisogna intervenire per salvare l'archivio stesso dalla dispersione. Nei paesi latini d'ogni continente la difficoltà consiste non tanto nell'incuria degli eredi (che spesso anzi fanno sforzi encomiabili per salvare i documenti), quanto nell'assenza di enti o istituzioni che si prendano cura del riordino, della conservazione e dell'apertura al pubblico 
dell'archivio. Ciascuno di questi tre passi è essenziale, ma richiede lavoro, spazi e personale. Beni rari, soprattutto in tempi di tagli ai bilanci.

Mi limiterò qui a illustrare il salvataggio dell'archivio di un giurista contemporaneo di Montevideo, cioè di quell'Eduardo Couture protagonista delle pagine precedenti. Il successo dell'impresa è stato favorito da alcune caratteristiche dell'Uruguay. Anzitutto, questo Stato ha avuto giuristi di rilevanza internazionale, con rapporti internazionali ramificati e intensi. In secondo luogo, poiché questi giuristi erano anche avvocati, i loro archivi erano stati tenuti in buon ordine nel corso di decenni da solerti segretarie: situazione pressoché impensabile per un professore tout court. In terzo luogo, la piccola dimensione dell'Uruguay evita la dispersione territoriale degli archivi privati: i principali archivi sono di fatto concentrati nella sola città di Montevideo. Negli Stati più vasti, invece, i trasferimenti di sede dei professori hanno in genere effetti devastanti sulla conservazione delle loro carte.

Nel 1998 venne costituito a Montevideo un «Grupo de trabajo para la preservación de archivos jurídicos uruguayos», sotto gli auspici della Facoltà di Giurisprudenza de la Universidad de la República, ${ }^{\mathbf{8 8}}$ di cui ho l'onore di far parte. La nostra attività è consistita dapprima nel riordinare i documenti conservati nell'archivio di Couture e, poi, nel registrarli su un supporto ottico.

Nel marzo 2003 venne finalmente prodotto e distribuito il CD-Rom intitolato Archivo privado del Dr. Eduardo J. Couture, che sfruttava tutte le potenzialità di questo supporto informatico. Su di esso erano infatti riprodotti testi, immagini e anche suoni. La parte testuale comprende le carte dell'archivio Couture, cioè le lettere, le memorie personali e quelle di viaggio (di cui si è avuto un saggio nelle pagine precedenti), i documenti sulle sue attività accademiche, culturali e professionali, i ritagli di giornali e ogni altra documentazione a stampa che Couture aveva ritenuto utile conservare. La parte grafica comprende la riproduzione delle fotografie che documentano i contatti culturali e professionali, nonché alcuni momenti famigliari della vita di Couture. Infine, poiché la radio di Montevideo conservava la registrazione di un discorso di Couture su Trabajo y justicia, anche questo documento sonoro è stato incluso nel CD-Rom in esame.

Però la digitalizzazione non presenta solo gli aspetti positivi finora visti. Con essa la conservazione dei documenti è di certo realizzabile con maggior

Cfr. supra, nota 27. 
ampiezza che con la carta stampata, ma la loro consultazione nel tempo, a lungo termine, si rivela più complessa proprio a causa dell'informatica. Oggi si può consultare senza difficoltà un'edizione stampata nel Seicento, però non sono più consultabili $\mathrm{i}$ nostri stessi supporti informatici di dieci o vent'anni fa. Per i «floppy disks» non sono neppure più reperibili le corrispondenti unità di lettura, per i cd. «dischetti» bisogna procurarsi un lettore esterno, perché gli odierni computer portatili non ne prevedono più l'uso.

$\mathrm{Ma}$ a questi problemi strumentali, cioè di hardware, si aggiungono quelli dei programmi, cioè di software. Dopo un certo periodo, i programmi applicativi (e anche quelli di base, che li fanno funzionare) vengono modificati più volte, e poi abbandonati a favore di altri programmi non necessariamente compatibili con i programmi anteriori. A quel punto il nostro CDRom conserva le informazioni, ma l'utente non riesce più ad accedere ad esse: lo storage è ottimo, ma il retrieval non è più possibile.

Proprio il CD-Rom dell'archivio Couture dimostra che queste difficoltà tecniche non sono un esempio teorico. Quel CD-Rom è stato prodotto tredici anni fa, e oggi il programma statunitense che lo fa funzionare non è più gestito dalla società uruguaiana che ne ha acquistato la licenza per realizzare il CD-Rom. In altre parole, presto potremmo trovarci nella situazione di aver salvato e moltiplicato l'archivio Couture, ma di avere gravi difficoltà nell'accedere ai documenti salvati.

Questa difficoltà insita nella digitalizzazione applicata alle biblioteche e agli archivi è nota da decenni agli specialisti. Infatti le biblioteche devono gestire una quantità esponenzialmente crescente di testi digitalizzati, in parte perché li producono esse stesse (ad esempio, per salvaguardare i propri testi rari), in parte perché un numero crescente di testi viene prodotto soltanto in forma digitalizzata. Questa compresenza di carta stampata e documenti digitalizzati ha ormai trasformato le grandi biblioteche tradizionali in «biblioteche ibride», con tutta una serie di esigenze (e di costi) loro propri. ${ }^{89}$ Basti qui ricordare che $\mathrm{i}$ testi giuridici sono $\mathrm{i}$ più adatti alla digitalizzazione, perché numerosi, vasti, ma caduchi nel tempo, e che quindi lo storico e il comparatista si troveranno sempre più spesso a dover cercare il materiale di

89 Sulla «hybrid library» esiste ormai un'estesa letteratura. Un quadro generale è nel testo bilingue di Kempf (2013). Cfr. inoltre Benvenuti/Morriello 2006, Marquardt/SalaRELLi 2005, FogLieni (2003). 
diritto positivo in banche di dati non necessariamente coordinate, anche dal punto di vista del software.

Mi auguro di aver fornito una prima idea del campo di ricerca e dell'interesse che gli archivi personali e, più in generale, la storia contemporanea del diritto possono presentare anche per il comparatista. Per me, l'aspetto più appassionante di questa ricerca è proprio quello che ad essa viene talora rimproverato dagli storici «classici»: la mancanza di distanza, cioè la vicinanza (ritenuta eccessiva) dell'oggetto. Ma proprio questo è per me il suo aspetto più vivo: l'oggetto è vicino; nella ricerca si incontrano volti noti ed eventi di cui si è parlato con i vecchi maestri, e nelle carte quel colloquio continua anche quando i vecchi maestri non sono più con noi. Insomma, si partecipa in prima persona alla conservazione d'un frammento della memoria storica del tempo in cui viviamo. Ed è naturale che sia così: la storia contemporanea la facciamo noi.

\section{Bibliografia}

Altamira y Crevea, Rafael (2007), Mi viaje a América. Libro de documentos, Oviedo: Universidad de Oviedo, XXVI, 358 pp. (ristampa dell'originale del 1911)

Ascarelli, Tullio (1949), Sguardo sul Brasile, Milano: Giuffrè, 218 pp.

Benvenuti, Nicola, Rossana Morriello (2006), Gestione delle raccolte e cooperazione nella biblioteca ibrida, Firenze: Firenze University Press, $112 \mathrm{pp}$.

Beviláqua, Clóvis (1893), Resumo das lições de legislação comparada sobre o direito privado, Recife: Typographia de F. P. Boulitreau, $159 \mathrm{pp}$.

Beviláqua, Clóvis (1897), Licções de legislação comparada, Bahia: Ed. José Luiz da Fonseca Magalhães, 295 pp. (più 6 pp. n.n. con Adições, Índice e Correcções)

Beviláqua, Clóvis (1903), O desenvolvimento do estudo da legislação comparada, Recife

Beviláqua, Clóvis (1906a), As funções da legislação comparada, Recife

Beviláqua, Clóvis (1906b), Princípios elementares de direito internacional privado, Bahia, ora ristampati Rio de Janeiro: Editora Rio, $368 \mathrm{pp}$.

Beviláqua, Clóvis (1935), Direito internacional privado, in: Revista de crítica judiciária, Rio de Janeiro

Biagini, Hugo Edgardo (ed.) (1985), El movimiento positivista argentino, Buenos Aires: Editorial de Belgrano, $590 \mathrm{pp}$.

Bobbio, Norberto (1990), Profilo ideologico del '900, Milano: Garzanti

Bobbio, Norberto (2007), Dalla struttura alla funzione, Roma, Bari: Laterza

Bruera, José Juan (1958), Doce años de correspondencia con Couture, Montevideo: Biblioteca de publicaciones oficiales de la Facultad de Derecho, 37 pp. 
Calamandrei, Piero (1936), Introduzione allo studio sistematico dei provvedimenti cautelari, Padova: Cedam, XI, 162 pp.

Calamandrei, Piero (1949), Scoperta di un pittore: Pedro Figari, in: Il Ponte V, n. 7 , pp. $903 \mathrm{~s}$.

Calamandrei, Piero (1954), Elogio dei giudici scritto da un avvocato, 3 ed., Firenze: Le Monnier, XXVI, 406 pp.

Calamandrei, Piero (1956), Eduardo J. Couture, in: Rivista di diritto processuale, XI, 245-250

Calamandrei, Piero (1957), Homenaje a Piero Calamandrei, in: Boletín del Instituto de Derecho Procesal, n. 7, 175 pp.

Cardim, Carlos Henrique (ed.) (2001), Bobbio no Brasil. Um retrato intelectual, Brasília, São Paulo: Universidade de Brasília, Imprensa Oficial do Estado, $160 \mathrm{pp}$.

Cossio, Carlos (1939), La plenitud del orden jurídico y la interpretación judicial de la ley, Buenos Aires: Losada, 220 pp.

Couture, Eduardo J. (1947), Gilberto Freyre y la sociología brasileña, Montevideo

Couture, Eduardo J. (1949), Los mandamientos del abogado, Buenos Aires: Depalma, 59 pp.

Couture, Eduardo J. (1953), La Comarca y el Mundo, Montevideo, 146 pp.

Couture, Eduardo J. (1954), Garanzia costituzionale del «dovuto processo legale», in: Rivista di diritto processuale, IX, 81-101

Couture, Eduardo (1957a), Prólogo a la edición argentina del «Elogio de los jueces escrito por un abogado", in: Boletín del Instituto de Derecho Procesal, n. 7 (Homenaje a Piero Calamandrei), 25-29

Couture, Eduardo J. (1957b), La escuela italiana de derecho, in: Boletín del Instituto de Derecho Procesal, n. 7 (Homenaje a Piero Calamandrei), 31-42

Couture, Eduardo J. (1957c), Due libri in uno, tradotto da Mauro Cappelletti, in: Rivista di diritto processuale, XII, 64-67

Del Vecchio, Giorgio (1930), Filosofía del derecho. Traducción, prólogo y extensas adiciones por Luis Recaséns Siches, Tomo II (Historia de la doctrinas), Barcelona: Bosch, 316 pp.

Del Vecchio, Giorgio (1934a), La crisi della scienza del diritto, 2. ed., Roma: Rivista Internazionale di Filosofia del Diritto, $30 \mathrm{pp}$.

Del Vecchio, Giorgio (1934b), Il problema delle fonti del diritto positivo, in: Rivista Internazionale di Filosofia del Diritto, XIV, 184-198

Del Vecchio, Giorgio (1935), Filosofía del derecho, 2 ed., vol. 1, Barcelona: Bosch Del Vecchio, Giorgio (1936a), Filosofía del derecho, 2 ed., vol. 2, Barcelona: Bosch Del Vecchio, Giorgio (1936b), Lezioni di filosofia del diritto, 3 ed. riv., Roma: Rivista Internazionale di Filosofia del Diritto, VIII, 399 pp.

Del Vecchio, Giorgio (1936c), Il problema delle fonti del diritto positivo, 2 ed. riv., Roma: Rivista Internazionale di Filosofia del Diritto, $21 \mathrm{pp.}$

Del Vecchio, Giorgio (1938), Il problema delle fonti del diritto positivo, 2 ed. riv., Roma: Rivista Internazionale di Filosofia del Diritto, 30 pp. 
Drago, Luigi M. (1890), I criminali-nati. Con introduzioni di Fr. Ramos Mejía e Cesare Lombroso, Torino: Bocca, 135 pp.

Filippi, Alberto, Celso Lafer (2006), El pensamiento de Bobbio en la cultura iberoamericana, Buenos Aires: Fondo de Cultura Económica, 212 pp.

Foglieni, Ornella (2003), La biblioteca ibrida, Verso un servizio informativo integrato, Bibliografica, Milano, 341 pp.

Homenaje a Piero Calamandrei (1957), Introducción de Santiago Sentís Melendo, Santa Fe: Universidad Nacional del Litoral, 175 pp.

Jiménez de AsúA, Luis (1944), El criminalista, Buenos Aires: La Ley, 315 pp. (contiene anche: El derecho penal de los indios, 251-257

Jiménez de Asúa, Luis (1960), Lombroso, Buenos Aires: Perrot, 73 pp. (con bibliografia)

Kempf, Klaus (2013), Der Sammlungsgedanke im digitalen Zeitalter - L'idea della collezione nell'età digitale, Fiesole: Casalini Libri, 132 pp.

Laplaza, Francisco P. (1950a), Antecedentes de nuestro periodismo forense hasta la aparición de «La Revista Criminal» (1873) como introducción a la historia del derecho penal argentino, Buenos Aires: Depalma, 285 pp.

Laplaza, Francisco P. (1950b), Francisco Carrara, sumo maestro del derecho penal, Buenos Aires: Depalma, 56 pp.

Laplaza, Francisco P. (1951), El periodismo forense como fuente de conocimiento de la historia jurídica, in: Revista del Instituto de Historia del Derecho 3, 85-111

Laplaza, Francisco P. (1954), Las ideas penales de Alberdi en el «Fragmento preliminar al estudio del Derecho», Buenos Aires: Arayú, 84 pp.

Levaggi, Abelardo (1977), El cultivo de la historia jurídica en la ciudad de Buenos Aires (1876-1919), Buenos Aires: Perrot, 214 pp.

Levaggi, Abelardo (1978), Historia del derecho penal argentino, Buenos Aires: Perrot, $214 \mathrm{pp}$.

Losano, Mario G. (1997), Storia contemporanea del diritto e sociologia storica, Milano: Franco Angeli, 265 pp.

Losano, Mario G. (1998), Renato Treves, sociologo tra il Vecchio e il Nuovo mondo. Con il regesto di un archivio ignoto e la bibliografia di Renato Treves, Milano: Unicopli, VIII, $210 \mathrm{pp}$.

Losano, Mario G. (1999), L'emigrazione dei giuristi milanesi nella Montevideo d'anteguerra, in: Rivista trimestrale di diritto e procedura civile, n. 1, 219-226

Losano, Mario G. (2000a), Calamandrei, Couture e un archivio giuridico contemporaneo a Montevideo, in: Gli argomenti umani. Sinistra e innovazione, n. 2-3, 96-102

Losano, Mario G. (2000b), Un giurista tropicale. Tobias Barreto fra Brasile reale e Germania ideale, Roma, Bari: Laterza

Losano, Mario G. (2001a), Il filosofo e il processualista. Il carteggio tra Giorgio Del Vecchio e Eduardo J. Couture (1904-1956), in: Rivista internazionale di filosofia del diritto, n. 1, 3-46 
Losano, Mario G. (2001b) [ma 2003], La emigración universitaria en el archivio privado de Eduardo J. Couture (1904-1956) en Montevideo, in: Eugénio Dos Santos, Asociación de Historiadores Latinoamericanistas Europeos, Actas do XII Congreso Internacional de Ahila, Universidade do Porto (1999), vol. IV, Porto, 245-251

Losano, Mario G. (2002), Clóvis Beviláqua entre comparação e filosofia do direito, in: VII Congresso Brasileiro de Filosofia, João Pessoa: Anais, Governo do Estado, Tribunal de Justiça da Paraíba, Centro Universitario de João Pessoa (Brasil), 389-404

Losano, Mario G. (2006), Miguel Reale (1910-2006), in: Rivista Internazionale di Filosofia del Diritto LXXXIII/3, 465-476

Losano, Mario G. (2007), Os grandes sistemas jurídicos. Introdução aos sistemas jurídicos europeus e extra-europeus. Tradução di Marcela Varejão, São Paulo: Martins Fontes

Lozano, Godofredo (1889), La escuela antropológica y sociológica criminal ante la sana filosofía, La Plata

Marquardt, Luisa, Alberto Salarelli (2005), Comunicare la conoscenza ai tempi del web. Ruolo e responsabilità della biblioteca ibrida, Roma: Associazione Italiana Biblioteche, 168 pp.

Mattes, Heinz (1977), Luis Jiménez de Asúa. Vida y obra, Buenos Aires: Depalma, 67 pp.

Meira, Sílvio (1990), Clóvis Beviláqua. Sua vida. Sua obra, Fortaleza: Edições Universidade Federal do Ceará, 457 pp.

Mondolfo, Rodolfo (1946), Cesare Beccaria y su obra, Buenos Aires: Depalma, 117 pp.

Quesada, Ernesto (1908), El sociologo Enrico Ferri y sus conferencias argentinas, Buenos Aires: Menéndez, 124 pp.

Sarlo, Oscar (1998), Grupo de trabajo para la preservación de archivos jurídicos uruguayos, Montevideo, dattiloscritto

Stolleis, Michael (ed.) (1995), Juristen. Ein biographisches Lexikon, München: Beck

Tobal, Gastón Federico (1939), Nuestro nuevo derecho. Hacia una ley más humana y más justa, Buenos Aires: Rosso, $174 \mathrm{pp}$.

Treves, Renato (1939), Neo-hegelismo italiano y neo-kantismo alemán en el pensamiento jurídico contemporáneo, in: Revista de derecho, jurisprudencia y administración XXXVII, 97-100

Treves, Renato (1957), Eduardo Couture (1904-1956), in: Rivista internazionale di filosofia del diritto

Varejão, Marcela (2005), Il positivismo dall'Italia al Brasile. Sociologia del diritto, giuristi e legislazione, 1822-1936, Milano: Giuffrè, XI, 465 pp. 



\section{Contributors}

Alfons Aragoneses. Lecturer at the Universitat Pompeu Fabra in Barcelona. $\mathrm{PhD}$ at the University of Girona (2006).Visiting researcher at the Max Planck Institut für europäische Rechtsgeschichte from 2000 to 2003. Main research fields include contemporary legal history, history of comparative law, legal history of Franquismo. Now (2015) researching on collective memory and social identity.

Agostina Gentili is a history graduate (National University of Córdoba UNC), doctoral scholar (CONICET, School of Philosophy and Humanities Research Center - UNC) and Assistant Professor of Social and Economic History of Argentina and Theory and Techniques of Social Research (School of Arts, UNC). Her research focuses on family and childhood historiography. She studies the relationships between families and public authorities, researching custody of children at Courts for Minors in Córdoba between 1957 and 1974.

Mario G. Losano, after teaching at the State University of Milan, is now emeritus professor of Legal Philosophy and Legal Informatics at Università degli Studi del Piemonte Orientale, and professor at the Doctorate School of Law and Istitutions of the Università degli Studi, Turin. His main research interests are the German legal philosophy of the 19th century, the circulation of European legal ideas outside of Europe in the 19th century, as well as e-government and privacy protection. Bio-bibliography in: www.mariolosano.it

Daniela Marino is Full Professor for 20th Century Mexican History in the M.A. and Ph.D. Program in History and Ethnohistory at the National School of Anthropology and History (ENAH, Mexico). She is also a member of the National Research System (SNI-CONACYT). She holds a Ph.D. in History (El Colegio de Mexico) and a certificate in Legal Anthropology (INAH). Marino's main research interests include Mexican Indian town 
history in the 19th and 20th Centuries and the changes in the legal framework, particularly on the land property system (communal/private/ejidal), and indigenous participation at the different instances of justice administration.

Marcelo Neves is Full Professor of Public Law and Legal Theory at the University of Brasilia Law School. He is currently a visiting scholar at Yale Law School. He studied at the Law School of the Federal University of Pernambuco in Brazil (LLB and LLM) and at the Universities of Frankfurt am Main and Bremen in Germany (Dr. iur.). He was awarded the "Habilitation" at the University of Fribourg Law School in Switzerland. He was Research Fellow of the Alexander von Humboldt Foundation at the Universities of Frankfurt am Main, Hamburg, Berlin (Humboldt University) and Flensburg in Germany as well as at the LSE, DAAD Research Fellow at the University of Bremen, Jean Monnet Fellow at the European University Institute in Italy and Adam Smith Fellow at the University of Glasgow. He was Visiting Professor at the Universities of Frankfurt and Flensburg in Germany, the University of Fribourg in Switzerland and the University of Chile. His main fields of research are the problems of constitutionalism in world society between centers and peripheries, law, state and constitution from the viewpoint of systems theory and discourse theory, and transconstitutionalism.

Yolanda de Paz Trueba is Professor at the National University of Central Buenos Aires and holds a PhD in History. She is Permanent Researcher at the National Research Council (CONICET) in Argentina and in the Institute of Geography, History and Social Sciences/CONICET. Her research interests include the place of women in the public sphere in the late nineteenth and early twentieth century. Currently her concerns focus on addressing the social, political and institutional practices affecting children (especially girls) between 1880 and 1920 in the province of Buenos Aires.

María Rosario Polotto is doctoral scholar at the Law School of the Pontifical University of Argentina, and Assistant Professor of Legal History at the same University and at the University of Buenos Aires. Also she is a researcher of the Research Institute of Legal History. Her research interests are the history of law in the twentieth century, especially the civil law. 
Maria Rosa Pugliese is Full Professor of Legal History at the Faculty of Law of the Universitiy of Buenos Aires, Argentina. Doctor of Law from the same University. She is a researcher of the Research Institute of Legal History and Social Research Ambrosio Lucas Gioja (University of Buenos Aires). Her research interests are the history of law in the twentiety century, the process of legal secularisation, marriage and justice. She is also interested in the study of Indian Law in the Rio de la Plata.

Gustavo Silveira Siqueira, Ph.D., is Assistant Professor of Legal History at the Rio de Janeiro State University (UERJ), visiting researcher at the Brazilian National Library (PNAP-R Program FBN) and member of the program »JCE - FAPERJ« Rio de Janeiro. He works on the history of the workers' strike in Brazil.

Alessandro Somma, a Research fellow at the Max-Planck-Institute for Legal history in Frankfurt am Main, Germany until 2002, is currently professor of Comparative law and Pro-rector for cultural activities and town-gown relations at the University of Ferrara, Italy. He is a member of the International Academy of Comparative law and of the board of the Italian Association of Comparative law. His main research fields include Theory and History of comparative law, Nazi and fascist theory of law, European private law, Globalization and theory of the state, Sovereign debt restructuring. 



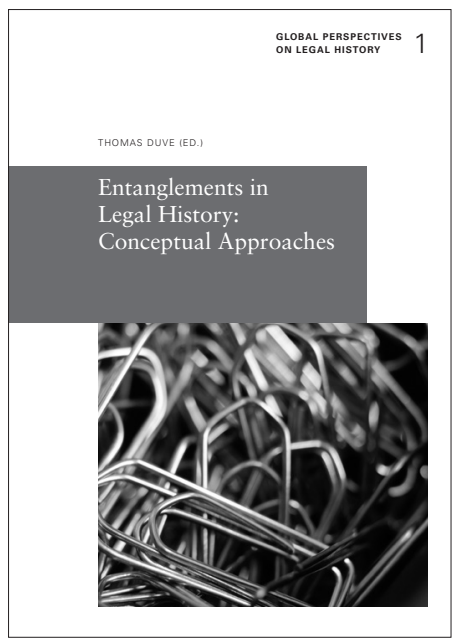

Thomas Duve (ed.)

Entanglements in Legal History: Conceptual Approaches

Global Perspectives on Legal History 1

Frankfurt am Main: Max Planck Institute for European Legal History 2014.

VII, 568 S., € 27,80 D

ISBN: 978-3-944773-00-1

Open Access Online Edition:

http://dx.doi.org/10.12946/gplh1

Global Perspectives on Legal History is a book series edited and published by the Max Planck Institute for European Legal History, Frankfurt am Main, Germany. As its title suggests, the series is designed to advance the scholarly research of legal historians worldwide who seek to transcend the established boundaries of national legal scholarship that typically sets the focus on a single, dominant modus of normativity and law.

The series aims to privilege studies dedicated to reconstructing the historical evolution of normativity from a global perspective.

It includes monographs, editions of sources, and collaborative works. All titles in the series are available both as premium print-on-demand and in the open-access format.

http:/global.rg.mpg.de 



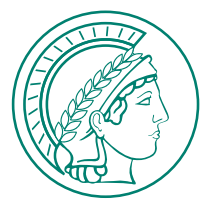

The present volume contains essays on the modernization of private law in Europe and Latin America from the viewpoint of legal history. Through a comparison between different forms of legal development in various normative und cultural contexts, the papers seek to open up new research perspectives on the concept of modernization in the field of private law.

$$
\text { http://global.rg.mpg.de }
$$

ISBN 978-3-944773-01-8 